Provided for non-commercial research and education use. Not for reproduction, distribution or commercial use.

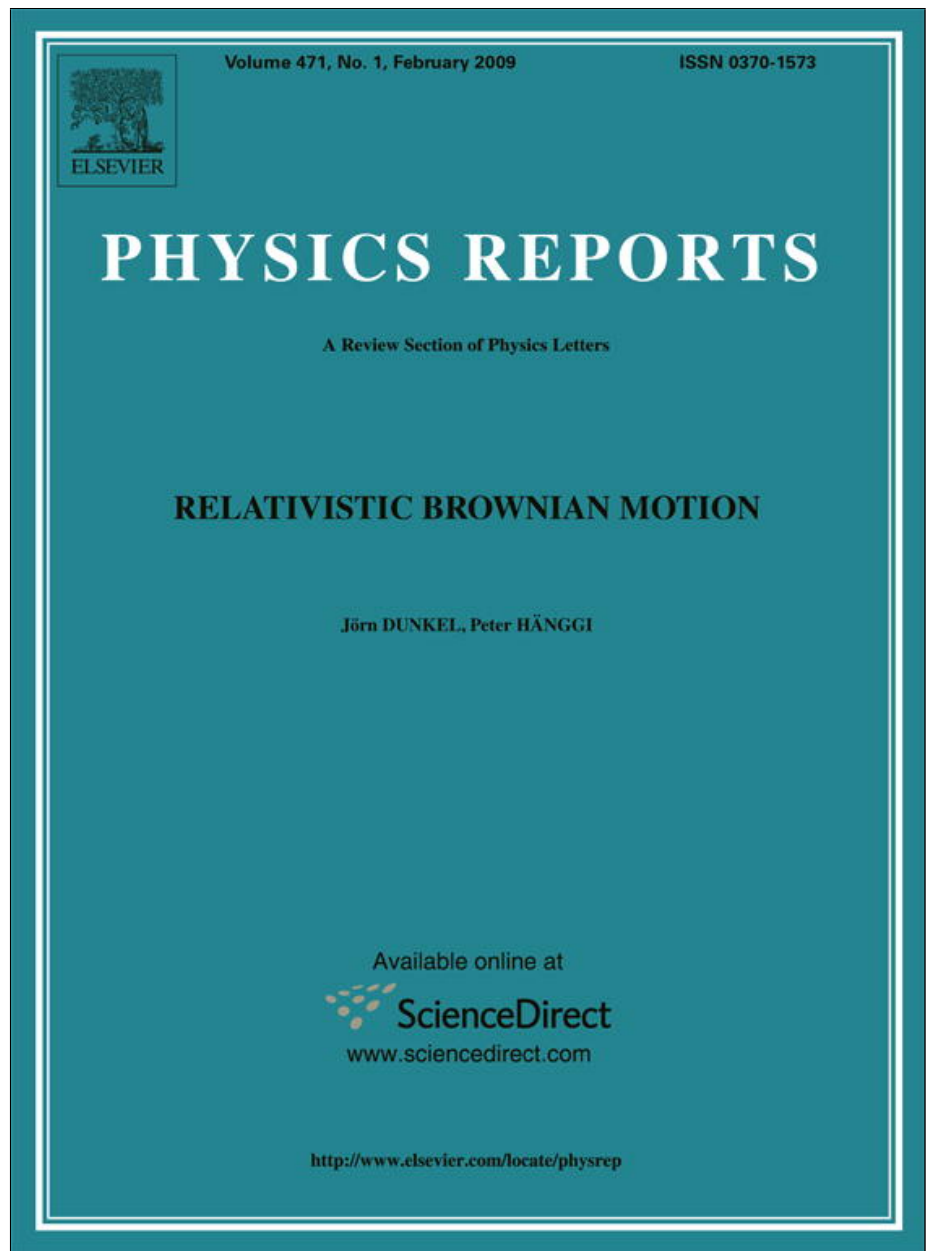

This article appeared in a journal published by Elsevier. The attached copy is furnished to the author for internal non-commercial research and education use, including for instruction at the authors institution and sharing with colleagues.

Other uses, including reproduction and distribution, or selling or licensing copies, or posting to personal, institutional or third party websites are prohibited.

In most cases authors are permitted to post their version of the article (e.g. in Word or Tex form) to their personal website or institutional repository. Authors requiring further information regarding Elsevier's archiving and manuscript policies are encouraged to visit:

http://www.elsevier.com/copyright 


\title{
Relativistic Brownian motion
}

\author{
Jörn Dunkel ${ }^{\mathrm{a}, *}$, Peter Hänggi ${ }^{\mathrm{b}}$ \\ ${ }^{a}$ Rudolf Peierls Centre for Theoretical Physics, University of Oxford, 1 Keble Road, Oxford OX1 3NP, United Kingdom \\ ${ }^{\mathrm{b}}$ Institut für Physik, Universität Augsburg, Universitätsstraße 1, D-86135 Augsburg, Germany
}

\section{A R T I C L E I N F O}

\section{Article history:}

Accepted 19 December 2008

Available online 6 January 2009

editor: J. Eichler

\section{PACS:}

02.50.Ey

05.40.-a

05.40.Jc

47.75.+f

\section{Keywords:}

Brownian motion

Special relativity

Stochastic processes

Relativistic Langevin equations

Fokker-Planck equations

Diffusion processes

Relativistic thermodynamics

\begin{abstract}
A B S T R A C T
Over the past one hundred years, Brownian motion theory has contributed substantially to our understanding of various microscopic phenomena. Originally proposed as a phenomenological paradigm for atomistic matter interactions, the theory has since evolved into a broad and vivid research area, with an ever increasing number of applications in biology, chemistry, finance, and physics. The mathematical description of stochastic processes has led to new approaches in other fields, culminating in the path integral formulation of modern quantum theory. Stimulated by experimental progress in high energy physics and astrophysics, the unification of relativistic and stochastic concepts has re-attracted considerable interest during the past decade. Focusing on the framework of special relativity, we review, here, recent progress in the phenomenological description of relativistic diffusion processes. After a brief historical overview, we will summarize basic concepts from the Langevin theory of nonrelativistic Brownian motions and discuss relevant aspects of relativistic equilibrium thermostatistics. The introductory parts are followed by a detailed discussion of relativistic Langevin equations in phase space. We address the choice of time parameters, discretization rules, relativistic fluctuationdissipation theorems, and Lorentz transformations of stochastic differential equations. The general theory is illustrated through analytical and numerical results for the diffusion of free relativistic Brownian particles. Subsequently, we discuss how Langevin-type equations can be obtained as approximations to microscopic models. The final part of the article is dedicated to relativistic diffusion processes in Minkowski spacetime. Since the velocities of relativistic particles are bounded by the speed of light, nontrivial relativistic Markov processes in spacetime do not exist; i.e., relativistic generalizations of the nonrelativistic diffusion equation and its Gaussian solutions must necessarily be non-Markovian. We compare different proposals that were made in the literature and discuss their respective benefits and drawbacks. The review concludes with a summary of open questions, which may serve as a starting point for future investigations and extensions of the theory.
\end{abstract}

(c) 2008 Elsevier B.V. All rights reserved.

\section{Contents}

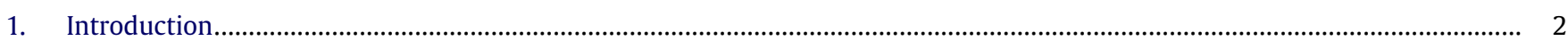

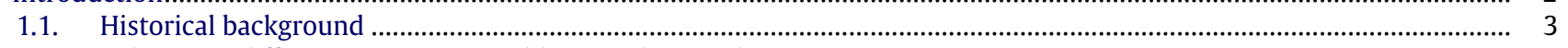

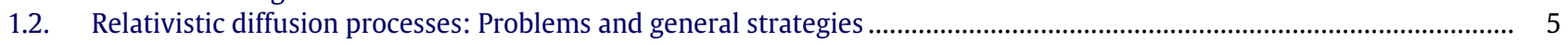

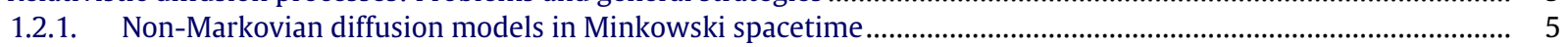

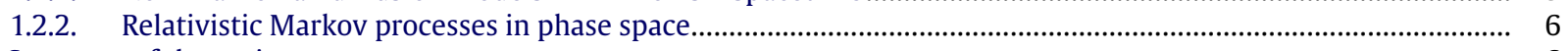

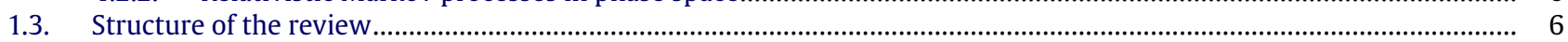

\footnotetext{
* Corresponding author.

E-mail addresses: jorn.dunkel@physics.ox.ac.uk (J. Dunkel), peter.hanggi@physik.uni-augsburg.de (P. Hänggi).

URLs: http://www-thphys.physics.ox.ac.uk/people/JornDunkel/ (J. Dunkel), http://www.physik.uni-augsburg.de/theo1/hanggi/ (P. Hänggi).
} 
2. Nonrelativistic Brownian motion.

2.1. Langevin and Fokker-Planck equations ...

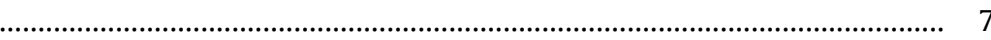

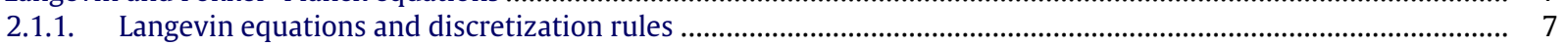

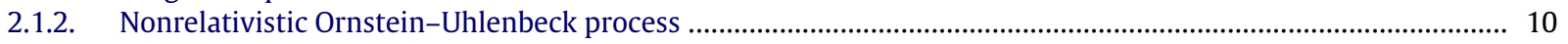

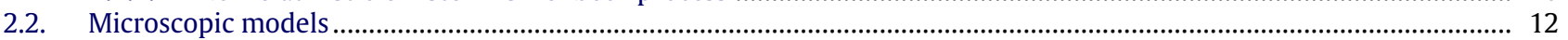

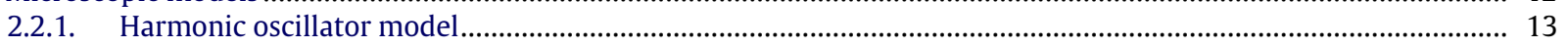

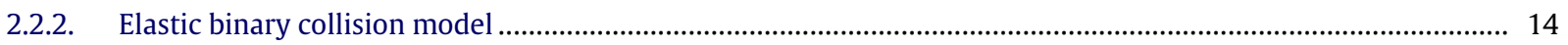

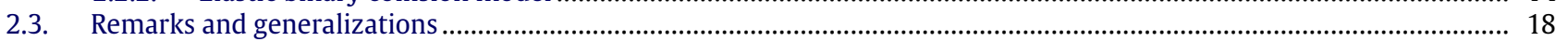

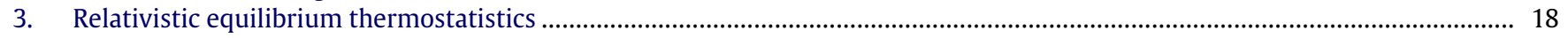

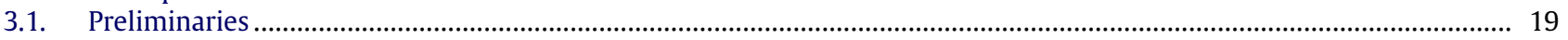

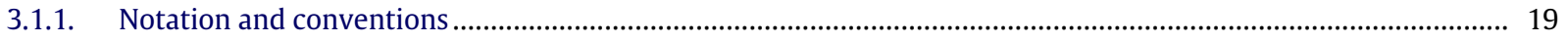

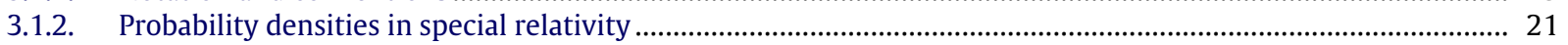

3.2. Stationary systems with confinement .................................................................................................................. 23

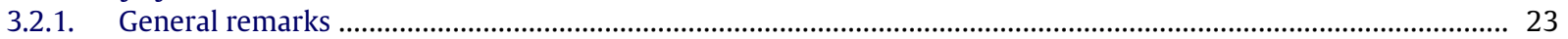

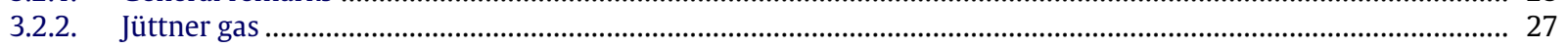

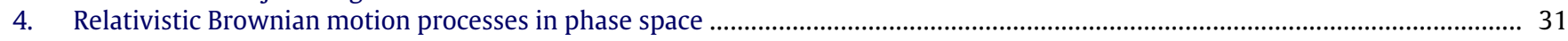

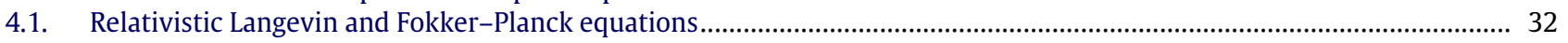

4.1.1. Relativistic Langevin equations: General construction principles ............................................................... 32

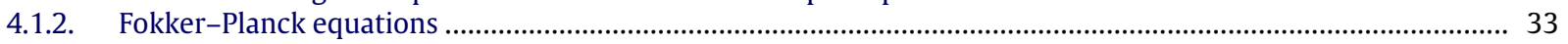

4.1.3. Free motions in an isotropic bath and Einstein relations............................................................................. 34

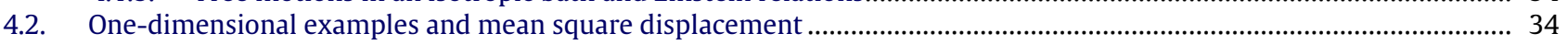

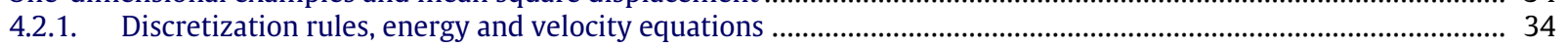

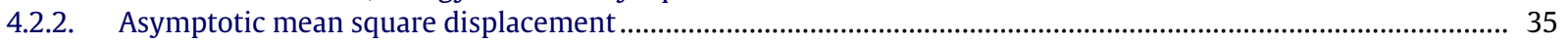

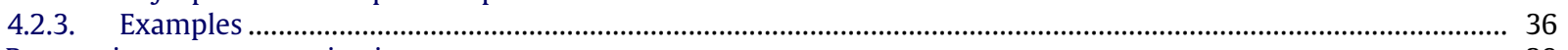

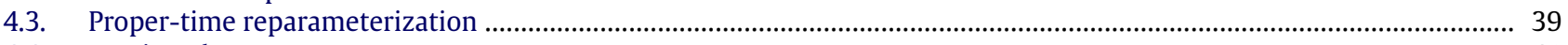

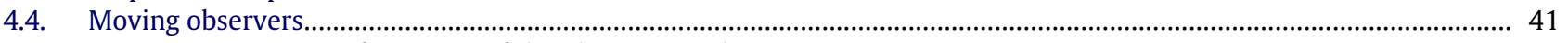

4.4.1. Lorentz transformation of the phase space density ...................................................................................

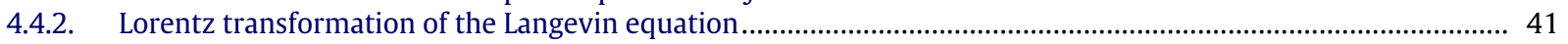

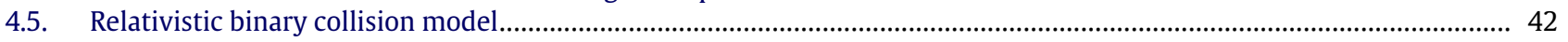

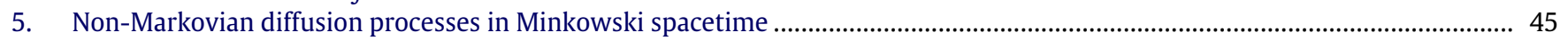

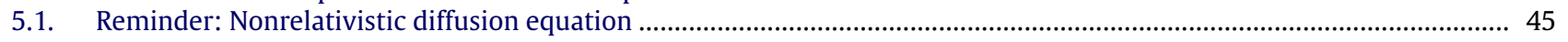

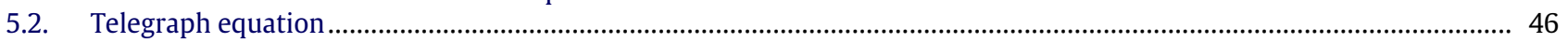

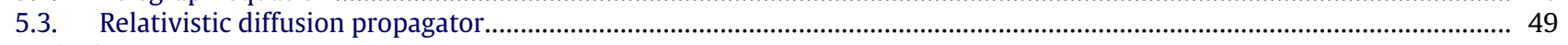

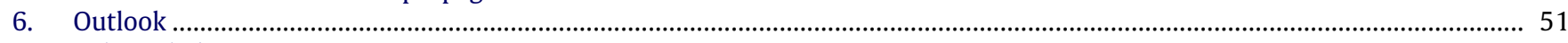

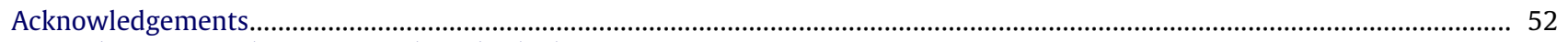

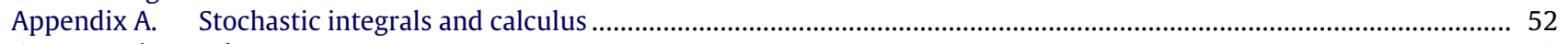

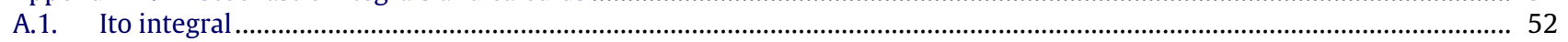

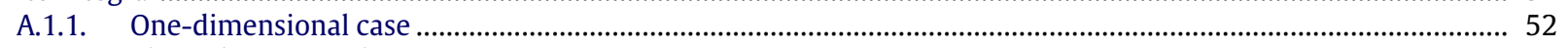

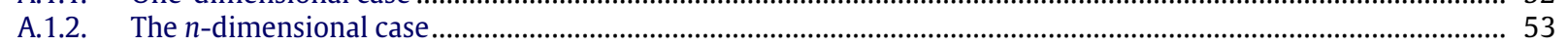

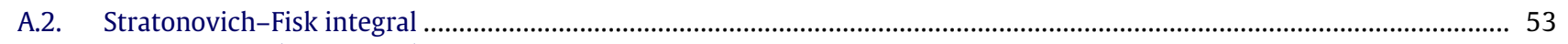

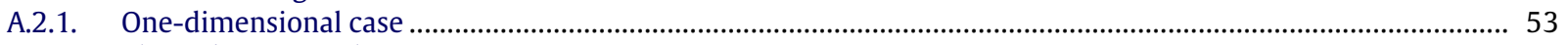

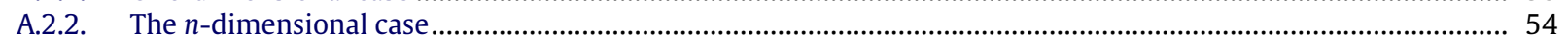

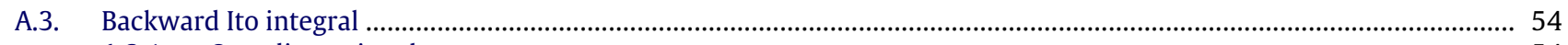

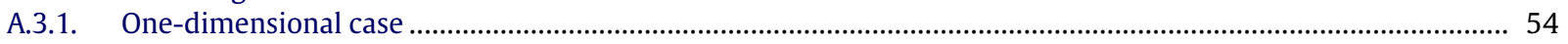

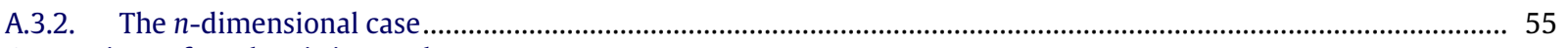

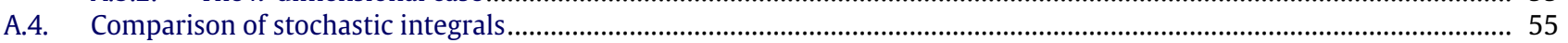

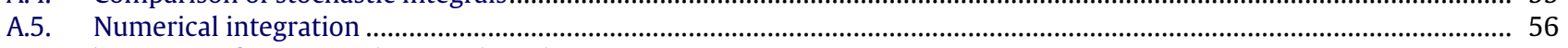

Appendix B. Surface integrals in Minkowski spacetime .................................................................................................. 56

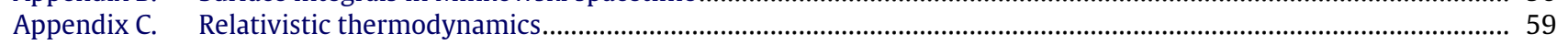

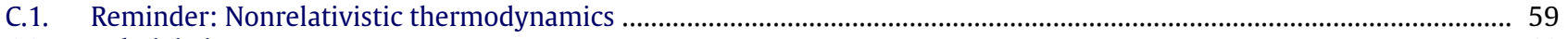

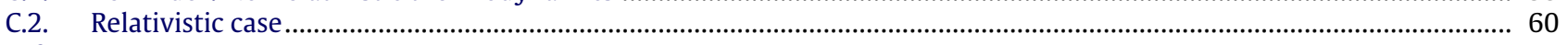

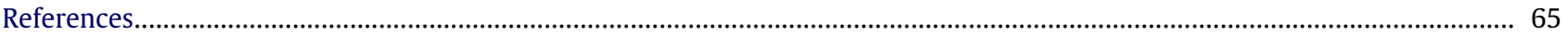

\section{Introduction}

In his annus mirabilis 1905 Albert Einstein published four manuscripts [1-4] that would forever change the world of physics. Two of those papers [2,3] laid the foundations for the special theory of relativity, while another one [4] solved the longstanding problem of classical (nonrelativistic) Brownian motion. ${ }^{1}$ Barring gravitational effects $[5,6]$, special relativity has proven to be the correct framework for describing physical processes on all terrestrial scales [7,8]. Accordingly, during the past century extensive efforts have been made to adapt established nonrelativistic theories such as, for example, thermodynamics, quantum mechanics or field theories [9] to the requirements of special relativity. Following this tradition, the present review focuses on recent progress in the theory of special relativistic Brownian motion and diffusion processes [10-34].

${ }^{1}$ Einstein's first paper [1] provided the explanation for the photoelectric effect. 


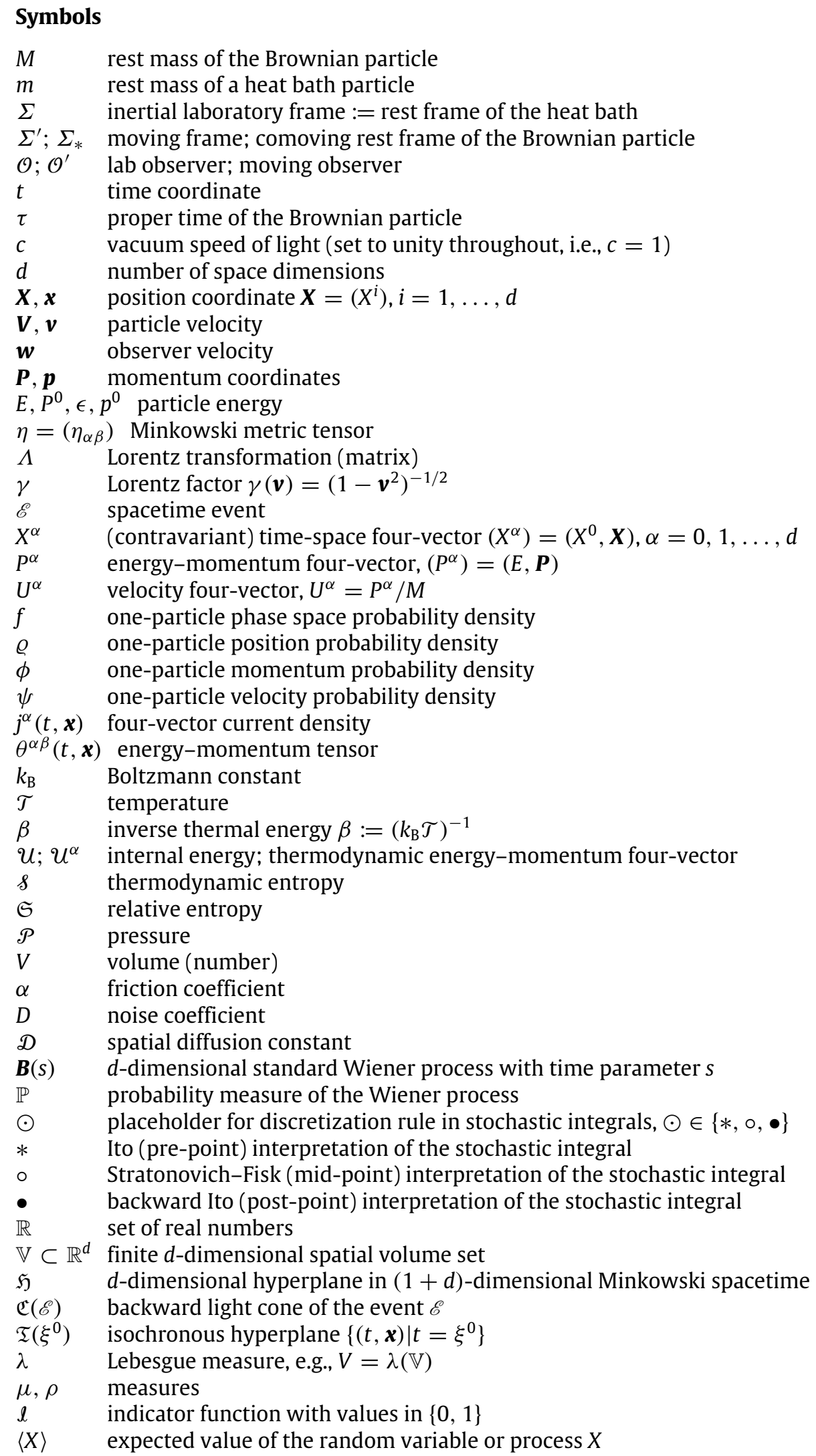

\subsection{Historical background}

Historically, the term 'Brownian motion' refers to the irregular dynamics exhibited by a test particle (e.g., dust or pollen) in a liquid environment. This phenomenon, already mentioned in 1784 by the Dutch physician Ingen-Housz [35,36], was first 
analyzed in detail by the Scottish botanist Robert Brown [37] in 1827. About eighty years later, Sutherland [38], Einstein [4] and von Smoluchowski [39] were able to theoretically explain these observations. They proposed that Brownian motion is caused by quasi-random, microscopic interactions with molecules forming the liquid. In 1909 their theory was confirmed experimentally by Perrin [40], providing additional evidence for the atomistic structure of matter. During the first half of the 20th century the probabilistic description of Brownian motion processes was further elaborated in seminal papers by Langevin [41,42], Fokker [43], Planck [44], Klein [45], Uhlenbeck and Ornstein [46] and Kramers [47]. ${ }^{2}$

In parallel with these early theoretical studies in the field of physics, outstanding mathematicians like Bachelier [50], Wiener [51-53], Kolmogoroff [54-56], Feller [57], and Lévy [58,59] developed a rigorous basis for the theory of Brownian motions and stochastic processes. Between 1944 and 1968 their groundbreaking work was complemented by Ito [60,61], Gihman [62-64], Fisk [65,66] and Stratonovich [67-69], who introduced and characterized different types of stochastic integrals or, equivalently, stochastic differential equations (SDEs). SDEs present a very efficient tool for modeling random processes and their analysis has attracted an ever-growing interest over the past decades [70-76]. ${ }^{3}$ Nowadays, the modern theory of stochastic processes goes far beyond the original problem considered by Einstein and his contemporaries, and the applications cover a wide range of different areas, including physics [77-84], biology [85,86], economy and finance [87-89].

A central topic of this review concerns the question of how SDE-based Brownian motion models can be generalized within the framework of special relativity. In the physics literature [75], SDEs are often referred to as Langevin equations [41, 42], and we shall use both terms synonymously here. From a mathematical perspective, SDEs [74] determine well-defined models of stochastic processes; from the physicist's point of view, their usefulness for the description of a real system is $a$ priori an open issue. Therefore, the derivation of nonrelativistic Langevin equations from microscopic models has attracted considerable interest over the past sixty years [21,90-96]. Efforts in this direction not only helped to clarify the applicability of SDEs to physical problems but led, among others, also to the concept of quantum Brownian motion [92,97-110]. ${ }^{4}$

If one aims at generalizing the classical Brownian motion concepts to special relativity, then several elements from relativistic equilibrium thermodynamics and relativistic statistical mechanics play an important role. More precisely, thermostatistic principles govern the stationary behavior of Brownian particles and, thus, impose constraints on the structure of relativistic Langevin equations. The first papers on relativistic thermodynamics were published in 1907 by Planck [120,121] and Einstein [122]. A main objective of theirs was to identify the Lorentz transformation laws of thermodynamic variables (temperature, pressure, etc.). ${ }^{5}$ In 1963 the results of Einstein and Planck became questioned by Ott [127], whose work initiated an intense debate about the correct relativistic transformation behavior of thermodynamic quantities [128-177]. ${ }^{6}$ However, as clarified by van Kampen [150] and Yuen [178], the controversy surrounding relativistic thermodynamics can be resolved by realizing that thermodynamic quantities can be defined in different, equally consistent ways.

While some authors considered relativistic thermodynamics as a purely macroscopic theory, others tried to adopt a more fundamental approach by focusing on relativistic equilibrium statistical mechanics. Early pioneering work in the latter direction was provided by Planck's students, von Mosengeil [184] and von Laue [185], and his collaborator Jüttner [186], who derived in 1911 the relativistic generalization of Maxwell's velocity distribution [187]. ${ }^{7}$ Research on relativistic equilibrium thermostatistics experienced its most intense phase between 1960 and 1970 [137,138,146,150,159,203-219]. An excellent exposition on the conceptual foundations and difficulties of relativistic statistical mechanics was given by Hakim [220-222] in 1967. Over the past years, the field has continued to attract interest [22,191,195-202,223-242].

The recurring debate on relativistic thermostatistics can be traced back to the difficulty of treating many-particle interactions in a relativistically consistent manner. ${ }^{8}$ In nonrelativistic physics, interactions may propagate at infinite speed, i.e., they can be modeled via instantaneous interaction potentials which enter additively in the Hamilton function; from

\footnotetext{
2 Excellent early reviews are given by Chandrasekhar [48], and Wang and Uhlenbeck [49].

3 The history of the mathematical literature on Brownian motions and stochastic processes is discussed extensively in Section 2.11 of Ref. [74]; see also Sections 2-4 in Nelson [71].

4 The vast literature on classical Brownian motion processes and their various applications in nonrelativistic physics is discussed in several survey articles [78-83,111-115]. Nonrelativistic generalizations of the standard theory as, for example, anomalous diffusion processes have been summarized in $[83,116,117]$, while review articles on nonrelativistic quantum Brownian motion can be found in $[107,108,118,119]$.

5 See also Pauli [123], Eddington [124], Tolman [125] and van Dantzig [126] for early discussions of this problem.

6 The pre-1970 literature on this disputed issue has been reviewed by Yuen [178] and Ter Haar and Wegland [179], see also Israel [177]; more recent surveys can be found in [180-183].

7 A general introduction to relativistic gases is given in Synge's textbook classic [188]. Relativistic generalizations of equipartition and virial theorems [189] are discussed by Pauli [123] and Einbinder [190]; more recent investigations of these two specific topics can be found in, for example, Refs. [191-193]. Equilibrium distributions for ideal relativistic quantum gases were also derived by Jüttner [194] in 1928. A few recent papers [22, 195-200] have raised doubts about the correctness of Jüttner's results [186,194], but relativistic molecular dynamics simulations confirm Jüttner's prediction [201,202], cf. discussion in Sections 3.2 .2 and 4.3 below.

8 Seminal contributions to the theory of relativistic many-particle interactions were provided by Fokker [243], Wheeler and Feynman [244,245] Pryce [246], Havas and Goldberg [247,248], and Van Dam and Wigner [249,250]. Over the past decades, several no-interaction theorems were proven [250253]. These forbid, within their respective qualifications, certain types of interaction models within the framework of special relativity. The mathematical structure of relativistic many-particle interactions was analyzed in detail by Arens and Babbitt [254]; various semi-relativistic approximations have been discussed, for example, in Refs. [255-257]. Kerner [258] has edited a reprint collection covering large parts of the pre-1972 literature on relativistic action-at-a-distance models; more recent contributions include [228,229,259-268]. An alternative, intensely studied method for describing relativistic interactions is based on the so-called constraint formalism [196,229,269-285]. The foundations of this approach were worked out by Dirac [269], who
} 
that point on, nonrelativistic statistical mechanics emerges without much difficulty [287,288]. Unfortunately, the situation becomes significantly more complicated in the relativistic case: Due to their finite propagation speed, relativistic interactions should be modeled by means of fields that can exchange energy with the particles [6]. These fields add an infinite number of degrees of freedom to the particle system. Eliminating the field variables from the dynamical equations may be possible in some cases, but this procedure typically leads to retardation effects, i.e., the particles' equations of motions become nonlocal in time $[220,221,244,245,249,250]$. Thus, in special relativity it is usually very difficult or even impossible to develop a consistent field-free Hamilton formalism of interacting particles.

In spite of the difficulties impeding a rigorous treatment of classical relativistic many-particle systems, considerable progress was made during the second half of the past century in constructing an approximate relativistic kinetic theory [167, 225,255,289-309] based on relativistic Boltzmann equations for the one-particle phase space probability density functions (PDFs). ${ }^{9}$ From such a kinetic theory, it is only a relatively small step to formulating a theory of relativistic Brownian motion processes in terms of Fokker-Planck equations and Langevin equations. While the relativistic Boltzmann equation [311, 312] is a nonlinear partial integro-differential equation for the PDF, Fokker-Planck equations are linear partial differential equations and, therefore, can be more easily solved or analyzed [73].

The present article focuses primarily on relativistic stochastic processes that are characterized by linear evolution equations for their respective one-particle (transition) PDFs. The corresponding phenomenological theory of relativistic Brownian motion and diffusion processes has experienced considerable progress during the past decade, with applications in various areas of high-energy physics [315-322] and astrophysics [323-327]. From a general perspective, relativistic stochastic processes provide a useful approach whenever one has to model the quasi-random behavior of relativistic particles in a complex environment. Therefore, it may be expected that relativistic Brownian motion and diffusion concepts will play an increasingly important role in future investigations of, e.g., thermalization and relaxation processes in astrophysics [323-326] or high-energy collision experiments [315,316,318,319,328,329].

\subsection{Relativistic diffusion processes: Problems and general strategies}

According to our knowledge, the first detailed mathematical studies on relativistic diffusion processes were performed independently by Łopuszaǹski [330], Rudberg [331], and Schay [332] between 1953 and 1961. In the 1960s and 70s their pioneering work was further elaborated by Dudley who published a series of papers [333-336] that aimed at providing an axiomatic approach to Lorentz invariant Markov processes [74] in phase space. Independently, a similar program was pursued by Hakim [220-222,337,338], whose insightful analysis helped to elucidate the conceptual subtleties of relativistic stochastic processes [338]. Dudley (Theorem 11.3 in [333]) and Hakim (Proposition 2 in [338]) proved the non-existence of nontrivial ${ }^{10}$ Lorentz invariant Markov processes in Minkowski spacetime, as already suggested by Łopuszaǹski [330]. This fundamental result implies that it is difficult to find acceptable relativistic generalizations of the well-known nonrelativistic diffusion equation $[287,339]$

$$
\frac{\partial}{\partial t} \varrho=D \nabla^{2} \varrho,
$$

where $\mathscr{D}>0$ is the diffusion constant and $\varrho(t, \boldsymbol{x}) \geq 0$ the PDF for the particle positions $\boldsymbol{x} \in \mathbb{R}^{d}$ at time $t$. In order to circumvent this 'no-go' theorem for relativistic Markov processes in spacetime, one usually adopts either of the following two strategies ${ }^{11}$ :

- One considers non-Markovian diffusion processes $\boldsymbol{X}(t)$ in Minkowski spacetime [10,23,365-367].

- One constructs relativistically acceptable Markov processes in phase space by considering not only the position coordinate $\boldsymbol{X}(t)$ of the diffusing particle, but also its momentum coordinate $\boldsymbol{P}(t)$ [11-22,24,26,31,32,220-222,332-338].

\subsubsection{Non-Markovian diffusion models in Minkowski spacetime}

A commonly considered 'relativistic' generalization of Eq. (1) is the telegraph equation [10,365-367]

$$
\tau_{v} \frac{\partial^{2}}{\partial t^{2}} \varrho+\frac{\partial}{\partial t} \varrho=D \nabla^{2} \varrho
$$

\footnotetext{
aimed at constructing a relativistic quantum theory for interacting many-particle systems [250-253]. For a detailed discussion of relativistic many-particle theory, we refer to the insightful considerations in the original papers of Van Dam and Wigner [249,250] and Hakim [220-222,224] as well as to the recent review by Hakim and Sivak [286].

9 Comprehensive introductions to relativistic Boltzmann equations can be found in the textbooks by Stewart [310], de Groot et al. [311], and Cercignani and Kremer [312], or also in the reviews by Ehlers [313] and Andréasson [314].

10 A diffusion process is considered as 'nontrivial' if a typical path has a non-constant, non-vanishing velocity.

11 The mathematical interest in relativistic diffusion processes increased in the 1980s and 1990s, when several authors [340-362] considered the possibility of extending Nelson's stochastic quantization approach [363] to the framework of special relativity. These studies, although interesting from a mathematical point of view, appear to have relatively little physical relevance because Nelson's stochastic dynamics [363] fails to reproduce the correct quantum correlation functions even in the nonrelativistic case [364].

12 Masoliver and Weiss [10] discuss several possibilities of deriving Eq. (2) from different underlying models.
} 
where $\tau_{v}>0$ denotes a finite relaxation time scale. Unlike the classical diffusion equation (1), which is recovered for $\tau_{v}=0$, the telegraph equation (2) contains a second order time-derivative and, therefore, describes a non-Markovian process. While the classical diffusion equation (1) permits superluminal propagation speeds, the diffusion fronts described by Eq. (2) travel at finite absolute velocity $v=\left(\mathscr{D} / \tau_{v}\right)^{1 / 2}$; cf. the discussion in Section 5 below.

Historically, Eq. (2) was first obtained by Thomson (Lord Kelvin) in 1854 [365], while studying the signal transduction for the first transatlantic cable. The first probabilistic derivation of Eq. (2) for the one-dimensional case was given by Goldstein [366] in 1950. His approach was based on a so-called persistent random walk model originally introduced by Fürth [368,369] in 1917 as a paradigm for diffusive motion in biological systems and later also considered by Taylor [370] in an attempt to treat turbulent diffusion. ${ }^{13}$ In contrast to standard non-directed random walk models, which lead to the classical diffusion equation (1) when performing an appropriate continuum limit [74], the random jumps of a persistent walk take into account the history of a path by assigning a larger probability to those jumps that point in the direction of the motion before the jump [366,367]. Persistent random walk models can be used to describe the transmission of light in multiple scattering media [372] such as foams [373-375] and thin slabs [371,376]. Similarly, the telegraph equation (2) has been applied in various areas of physics over the past decades, e.g., to model the propagation of electric signals and heat waves. ${ }^{14}$

Moreover, an interesting connection between the free particle Dirac equation [382] and the telegraph equation (2) was pointed out by Gaveau et al. [383] in 1984: The solutions of both equations may be linked by means of an analytic continuation quite similar to the relation between the classical diffusion equation (1) and the free-particle Schrödinger equation in the nonrelativistic case. ${ }^{15}$ On the other hand, the telegraph equation (2) is not the only possible generalization of Eq. (1) and a rather critical discussion of Eq. (2) in the context of relativistic heat transport was given by van Kampen [153] in 1970. Section 5 below takes a closer look at the properties of Eq. (2) and also addresses potential alternatives [23,390].

\subsubsection{Relativistic Markov processes in phase space}

As an alternative to non-Markovian diffusion models in spacetime, one can consider relativistic Markov processes in phase space [11-22,24,26,31-34,220-222,332-338]. Typical examples are processes described by Fokker-Planck equations (FPEs) or Langevin equations [11-15,17,18,20,21,24-26,31-34,391-395]. Similar to the relativistic Boltzmann equation, relativistic FPEs in phase space can be used to describe non-equilibrium and relaxation phenomena in relativistic many-particle systems. FPEs can be derived from Langevin equations, as approximations to more general linear master equations [75,332] or by approximating the collision integrals in nonlinear Boltzmann equation by differential expressions that contain effective friction and diffusion coefficients [16,299]. In particular, the latter method was successfully applied in different areas of physics over the past decades, including plasma physics [299,396-408], high-energy physics [315-319,328,329], and astrophysics [324,325,409-412]. ${ }^{16}$ In the 1980s and 90s this approach was further elaborated [396-404] and several numerical methods for solving FPEs were developed [399,417-419]. ${ }^{17}$ Recent applications include the modeling of diffusion and thermalization processes in quark-gluon plasmas, as produced in relativistic heavy ion collision experiments [315,316,318,319, 328,329], as well as the description of complex high-energy processes in astrophysics [323-325,409-412].

A complementary approach towards relativistic stochastic processes in phase space starts from Langevin equations [11$15,17,18,20,21,24-26,31,32,391-395]$. Stochastic differential equations of the Langevin type yield explicit sample trajectories for the stochastic motion of a relativistic Brownian particle. Relativistic Langevin equations may either be postulated as phenomenological model equations [11,17] or obtained from more precise microscopic models by imposing a sequence of approximations [21]. Compared with the nonrelativistic case, the latter task becomes considerably more complicated due to the aforementioned conceptual and technical difficulties in formulating consistent relativistic manyparticle theories. The phenomenological Langevin approach to relativistic Brownian motion was initiated by Debbasch et al. [11] who, in 1997, proposed a simple relativistic generalization of the classical Ornstein-Uhlenbeck process [46], representing a special limit cases of a larger class of relativistic Langevin processes $[15,19,26]$. From a practical point of view, relativistic Langevin equations provide a useful tool for modeling the dynamics of relativistic particles in a random environment, because these SDEs may be simulated by using well-established Monte-Carlo techniques that are numerically robust and efficient $[76,89,420]$. Recent applications include the analysis of thermalization effects in quarkgluon plasmas [318,319,329,421] and ultrarelativistic plasma beam collisions [326].

\subsection{Structure of the review}

The present article intends to provide a comprehensive introduction to the theory of relativistic Brownian motions, with a particular emphasis on relativistic Langevin equations. For this purpose, the subsequent parts are organized as follows.

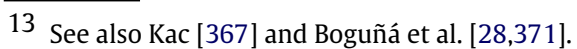

14 A detailed review of the pre-1990 research on heat waves was provided by Joseph and Preziosi [377,378], while more recent discussions and applications of Eq. (2) can be found in [10,27,29,320,321,379-381].

15 For further reading about path integral representations of the Dirac propagator we refer to the papers of Ichinose [384,385], Jacobson and Schulman [386], Barut and Duru [387], and Gaveau and Schulman [388]; see also footnote 7 in Gaveau et al. [383] and pp. 34-36 in Feynman and Hibbs [389].

16 Relativistic Fokker-Planck-type equations also played a role in the debate about whether or not the black body radiation spectrum is compatible with Jüttner's relativistic equilibrium distribution [191,413-416].

17 A main reason for the lively interest in relativistic FPEs at that time was the prospect of building plasma fusion reactors.
} 
Section 2 summarizes the Langevin theory of nonrelativistic Brownian motions in phase space. Section 3 discusses aspects of relativistic equilibrium thermostatistics as far as relevant for the subsequent discussion. Relativistic Langevin equations in phase space and their associated FPEs are considered in Section 4. Section 5 is dedicated to relativistic diffusion processes in Minkowski spacetime; as outlined above, such processes must necessarily be non-Markovian. Our review concludes with a summary of open questions in Section 6, which may serve as a starting point for future investigations and extensions of the theory. Since the topic of this article resides in the intersection between statistical and high-energy physics, we hope that the presentation is accessible for members of both communities. To keep the discussion as self-contained as possible, the article includes three appendices which summarize a few essentials about stochastic integrals, surface integrals in Minkowski spacetime and relativistic thermodynamics.

\section{Nonrelativistic Brownian motion}

This section summarizes basic definitions and mathematical tools, as well as relevant results from the nonrelativistic theory of nonrelativistic Brownian motions. In particular, the Langevin and Fokker-Planck equations considered in this part define the nonrelativistic limit case of the relativistic theory described Section 4. In Section 2.1 we shall briefly recall the general mathematical structure of Langevin and Fokker-Planck equations, the relevance of discretization rules and the choice of fluctuation-dissipation relations. Section 2.2 focuses on the question of how stochastic differential equations (SDEs) can be motivated and/or derived from microscopic models. As typical examples, the well-known harmonic oscillator model [90-96] and a recently proposed binary collision model [21] will be considered. In contrast to the oscillator model, the collision model can be generalized to the framework of special relativity, and its relativistic version will be discussed in Section 4.5 .

\subsection{Langevin and Fokker-Planck equations}

The condensed discussion in this part is primarily based on the papers of Uhlenbeck and Ornstein [46], Wang and Uhlenbeck [49], and Klimontovich [112]. For further reading about nonrelativistic stochastic processes and their numerous applications in physics and mathematics, we refer to the review articles of Chandrasekhar [48], Fox [111], Hänggi and Thomas [77], Bouchaud and Georges [116], Metzler and Klafter [117], Hänggi and Marchesoni [81], Frey and Kroy [82], or the textbooks references [74-76,422]. For conceptual clarity, we restrict ourselves to the simplest case where motions are confined to one space dimension $(d=1)$. The generalization to higher space dimensions is obvious.

\subsubsection{Langevin equations and discretization rules}

As a standard paradigm for Brownian motion, we consider the one-dimensional motion of a point-like Brownian particle (mass $M$ ), which is surrounded by a stationary homogeneous heat bath consisting, for example, of smaller liquid particles (mass $m \ll M$ ) at constant temperature $\mathcal{T}$. The inertial rest frame ${ }^{18} \Sigma$ of the heat bath will be referred to as lab frame hereafter. The position of the Brownian particle in $\Sigma$ at time $t$ is denoted by $X(t)$ and its velocity is given by $V(t):=\mathrm{d} X(t) / \mathrm{d} t$. The associated nonrelativistic momentum of the Brownian particle is defined by $P(t):=M V(t)$.

According to the Langevin picture of Brownian motion, the stochastic dynamics of the Brownian particle due to the interaction with the bath, and in the presence of a conservative external force field, $\mathcal{F}(t, x)$ can be described by the differential equations $[25,41,42,46,48,49,77,112,423]$

$$
\begin{aligned}
& \frac{\mathrm{d} X}{\mathrm{~d} t}=\frac{P}{M}, \\
& \frac{\mathrm{d} P}{\mathrm{~d} t}=\mathcal{F}(t, X)-\alpha(P) P+\mathscr{L}(P, t),
\end{aligned}
$$

complemented by the initial conditions ${ }^{19} X(0)=x_{0}$ and $P(0)=p_{0}$. The second term on the rhs of Eq. (3b) is a friction force, with the shape of the friction coefficient function $\alpha(p)>0$ depending on the microscopic details of the particle-bath interaction. The stochastic Langevin force

$$
\mathcal{L}(P(t), t)=[2 D(P)]^{1 / 2} \odot \zeta(t)
$$

reflects fluctuations in the surrounding heat bath. The symbol $\odot$ signals the choice of a specific discretization rule to be discussed in more detail below. The amplitude of the fluctuating force $\mathscr{L}$ is tuned by function $D(p)>0$. For a spatially inhomogeneous heat bath, the functions $\alpha$ and $D$ would also depend on the position coordinate $x$. The stochastic 'driving' function $\zeta(t)$ is often taken to be a Gaussian white noise, i.e., $\zeta(t)$ is characterized by:

$$
\begin{aligned}
& \langle\zeta(t)\rangle=0, \\
& \langle\zeta(t) \zeta(s)\rangle=\delta(t-s),
\end{aligned}
$$

18 By definition, the mean velocity of the heat bath particles vanishes in $\Sigma$.

19 Without loss of generality we fix the initial time $t_{0}=0$. 
with all higher cumulants being zero. In Eq. (4), the bracket $\langle\cdot\rangle$ symbolizes an average over all possible realizations of the noise process $\zeta(t)$.

In the mathematical literature [74,76], SDEs like the Langevin equation (3) are usually written in the differential notation

$$
\begin{aligned}
& \mathrm{d} X(t)=(P / M) \mathrm{d} t, \\
& \mathrm{~d} P(t)=\mathcal{F}(t, X) \mathrm{d} t-\alpha(P) P \mathrm{~d} t+[2 D(P)]^{1 / 2} \odot \mathrm{d} B(t) .
\end{aligned}
$$

Here, $\mathrm{d} X(t):=X(t+\mathrm{d} t)-X(t)$ denotes the position increment and $\mathrm{d} P(t):=P(t+\mathrm{d} t)-P(t)$ the momentum change. The random function $B(t)$ is a standardized one-dimensional Brownian motion or, equivalently, a standard Wiener process [51, 74,76,77], whose increments

$$
\mathrm{d} B(t):=B(t+\mathrm{d} t)-B(t)
$$

are defined to be stochastically independent ${ }^{20}$ and characterized by the Gaussian probability distribution

$$
\mathbb{P}\{\mathrm{d} B(t) \in[y, y+\mathrm{d} y]\}=(2 \pi \mathrm{d} t)^{-1 / 2} \exp \left[-y^{2} /(2 \mathrm{~d} t)\right] \mathrm{d} y ;
$$

i.e., the increments $d B(t)$ are independent random numbers drawn from a normal distribution with variance $d t$. From Eq. (5d) and the independence of the increments at different times $s \neq t$, it follows that

$$
\langle\mathrm{d} B(t)\rangle=0, \quad\langle\mathrm{~d} B(t) \mathrm{d} B(s)\rangle= \begin{cases}0, & t \neq s, \\ \mathrm{~d} t, & t=s,\end{cases}
$$

where now the expectation $\langle\cdot\rangle$ is taken with respect to the probability measure $\mathbb{P}$ of the Wiener process $B(t)$. The two different representations ( 3 ) and (5) may be connected by formally identifying

$$
\mathrm{d} B(t)=\zeta(t) \mathrm{d} t .
$$

In the remainder, we will write SDEs primarily in the differential notation of Eq. (5), which may also be viewed as a simple numerical integration scheme, cf., e.g., Ref. [89,420] and Appendix A.

It is worthwhile to summarize the physical assumptions, implicitly underlying Eqs. (3) and (5):

- The heat bath is spatially homogeneous and stationary; i.e., relaxation processes within the heat bath occur on time scales much shorter than the relevant dynamical time scales associated with the motion of the heavy Brownian particle. ${ }^{21}$

- Stochastic impacts between the Brownian particle and the constituents of the heat bath occur virtually uncorrelated.

- On a macroscopic level, the interaction between Brownian particle and heat bath is sufficiently well described by the friction coefficient $\alpha$ and the stochastic Langevin force $\mathcal{L}$.

- Eqs. (3) and (5) hold in the lab frame $\Sigma$, corresponding to the specific inertial system, where the average velocity of the heat bath particles vanishes for all times $t$.

In Section 2.2 we shall review how stochastic dynamical equations similar to Eqs. (3) and (5) can be derived or, at least, motivated by means of specific microscopic models.

Discretization rules. A stochastic force with a momentum dependent noise amplitude function $D(p)$ as in Eq. (5b) is usually referred to as 'multiplicative' noise. When considering SDEs that contain multiplicative noise terms, the specification of the discretization rule $\odot$ is necessary since, for fixed functions $\alpha(p)$ and $D(p)$, different discretization schemes in general lead to non-equivalent stochastic processes [74,76,77,422]; put differently, the values of the stochastic integral $P(t)$ defined by Eq. (5b) depend on the choice of discretization rule. This is the most essential difference compared with ordinary differential equations, whose integral curves (i.e., solutions) are independent of the underlying discretization scheme when taking the continuum limit $\mathrm{d} t \rightarrow 0 .^{22}$ The three most commonly considered discretization rules are the following ones [424] ${ }^{23}$ :

- The pre-point discretization of Ito $[60,61]$, denoted by “ $\odot=*$ ", is defined by computing the function $C(P)=[D(P)]^{1 / 2}$ at $P(t)$, i.e.,

$$
C(P) * \mathrm{~d} B(t):=C(P(t)) \mathrm{d} B(t) .
$$

- The post-point rule " $\odot=\bullet$ ", sometimes also referred to as kinetic [112] or backward Ito rule [424], is defined by evaluating the function $C(P)=[D(P)]^{1 / 2}$ at $P(t+\mathrm{d} t)$, i.e.,

$$
C(P) \bullet \mathrm{d} B(t):=C(P(t+\mathrm{d} t)) \mathrm{d} B(t) .
$$

\footnotetext{
20 This means that the joint probability density of an arbitrary collection of subsequent increments $\mathrm{d} B\left(t_{i}\right)$ is a product of the Gaussian distributions - see, for example, $[74,76]$ for a precise mathematical definition.

21 Interaction with a spatially inhomogeneous non-stationary heat bath can be modeled, e.g., by considering friction and noise amplitude functions of the form $\alpha(t, x, p)$ and $D(t, x, p)$.

22 For example, the discretization rule is irrelevant when the driving process $B(t)$ is a regular function, e.g., if $B(t)=\sin (\omega t) \operatorname{such}$ that $\mathrm{d} B(t)=\cos (\omega t) \mathrm{d} t$. If, however, the driving process is a strongly fluctuating function (of unbounded variation) as, for example, a Wiener (white noise) process, then different integration rules may yield non-equivalent trajectories; cf. Problem 2.29 in [74].

23 A brief summary of the different discretization rules and their consequences with regard to stochastic differential calculus is given in Appendix A.
} 
- The mid-point rule " $\odot=0$ " of Stratonovich [67-69] and Fisk [65,66] is defined by taking the mean value of the Ito and the backward Ito stochastic integral, i.e.,

$$
C(P) \circ \mathrm{d} B(t):=\frac{1}{2}[C(P) * \mathrm{~d} B(t)+C(P) \bullet \mathrm{d} B(t)] .
$$

From the mathematical point of view, the choice of the discretization rule reduces more or less to a matter of convenience $[74,76]$. To briefly illustrate this, consider Eq. (5) with post-point rule " $\odot=\bullet$ ", reading

$$
\begin{aligned}
& \mathrm{d} X(t)=(P / M) \mathrm{d} t, \\
& \mathrm{~d} P(t)=[\mathcal{F}(t, X)-\alpha(P) P] \mathrm{d} t+[2 D(P)]^{1 / 2} \bullet \mathrm{d} B(t) .
\end{aligned}
$$

Then, for each pair of sufficiently smooth functions $(\alpha(p), D(p))$, one can determine new friction coefficients $\alpha_{\circ \mid *}(p)$ such that the pairs $\left(\alpha_{\circ \mid *}(p), D(p)\right)$ describe exactly the same stochastic dynamics as Eq. (8) when combined with the corresponding discretization rule $\circ$ and $*$, respectively. Specifically, one can replace Eq. (8b) by the equivalent Stratonovich-Fisk SDE

$$
\begin{aligned}
& \mathrm{d} P(t)=\left[\mathcal{F}(t, X)-\alpha_{\circ}(P) P\right] \mathrm{d} t+[2 D(P)]^{1 / 2} \circ \mathrm{d} B(t), \\
& \alpha_{\circ}(p):=\alpha(p)-D^{\prime}(p) /(2 p),
\end{aligned}
$$

or, alternatively, by the equivalent Ito SDE

$$
\begin{aligned}
& \mathrm{d} P(t)=\left[\mathcal{F}(t, X)-\alpha_{*}(P) P\right] \mathrm{d} t+[2 D(P)]^{1 / 2} * \mathrm{~d} B(t), \\
& \alpha_{*}(p):=\alpha(p)-D^{\prime}(p) / p,
\end{aligned}
$$

where $D^{\prime}(p):=\mathrm{d} D(p) / \mathrm{d} p$. The modified friction coefficients in Eqs. (9b) and (10b) account for the fact that the three discretization rules are characterized by different conditional expectations, respectively:

$$
\begin{aligned}
& \left\langle[2 D(P)]^{1 / 2} \bullet \mathrm{d} B(t) \mid P(t)=p\right\rangle=D^{\prime}(p) \mathrm{d} t, \\
& \left\langle[2 D(P)]^{1 / 2} \circ \mathrm{d} B(t) \mid P(t)=p\right\rangle=D^{\prime}(p) \mathrm{d} t / 2, \\
& \left\langle[2 D(P)]^{1 / 2} * \mathrm{~d} B(t) \mid P(t)=p\right\rangle=0 .
\end{aligned}
$$

From a practical point of view, each of the three above mentioned discretization methods has its own merits and drawbacks: Ito's pre-point rule $(*)$ is particularly convenient for numerical simulations, but care is required when considering nonlinear transformations $G(P)$ of the momentum coordinate due to modifications of the differential calculus, cf. Appendix A. By contrast, if one adopts the Stratonovich-Fisk mid-point rule (o), then the transformation rules from ordinary differential calculus carry over, but it becomes more difficult to implement this mid-point rule in numerical simulations. The latter disadvantage also applies to the post-point rule employed in Eq. (5b). However, as we shall see next, the post-point rule $(\bullet)$ leads to a particularly simple form of the fluctuation-dissipation relation. ${ }^{24}$

Fokker-Planck equation. When studying SDEs of the type (5), one is typically interested in the probability

$$
f(t, x, p) \mathrm{d} x \mathrm{~d} p
$$

of finding the Brownian particle at time $t$ in the infinitesimal phase space interval $[x, x+\mathrm{d} x] \times[p, p+\mathrm{d} p]$. The non-negative phase space probability density function (PDF) $f(t, x, p) \geq 0$ of the Brownian particle is normalized at all times, i.e.

$$
1=\int \mathrm{d} x \mathrm{~d} p f(t, x, p), \quad \forall t>0 ;
$$

where, here and below, unspecified integrals range over the full phase space, position space, or momentum space, respectively. Given the phase space $\operatorname{PDF} f(t, x, p)$, the marginal momentum $\operatorname{PDF} \phi(t, p)$ and the marginal position $\operatorname{PDF} \varrho(t, x)$ are defined by

$$
\begin{aligned}
& \phi(t, p)=\int \mathrm{d} x f(t, x, p), \\
& \varrho(t, x)=\int \mathrm{d} p f(t, x, p) .
\end{aligned}
$$

Deterministic initial data $X(0)=x_{0}$ and $P(0)=p_{0}$ translate into the initial conditions

$$
\begin{aligned}
& f(0, x, p)=\delta\left(x-x_{0}\right) \delta\left(p-p_{0}\right), \\
& \phi(0, p)=\delta\left(p-p_{0}\right), \\
& \varrho(0, x)=\delta\left(x-x_{0}\right) .
\end{aligned}
$$

\footnotetext{
$\overline{24}$ The general conversion formulae for the different discretization rules are summarized in Appendix A.
} 
Adopting the post-point rule $(\bullet)$, the FPE describing the phase space density $f(t, x, p)$ of the stochastic process (5) is given by $^{25}$

$$
\frac{\partial f}{\partial t}+\frac{p}{M} \frac{\partial f}{\partial x}+\mathcal{F}(t, x) \frac{\partial f}{\partial p}=\frac{\partial}{\partial p}\left[\alpha(p) p f+D(p) \frac{\partial f}{\partial p}\right] .
$$

Eq. (15) is a linear partial differential equation in $f$, i.e., more general general solutions can be obtained by integrating the special solution with deterministic initial condition (14) over some arbitrary initial distribution $f_{0}\left(x_{0}, p_{0}\right)$. Moreover, the FPE (15) is of first order in time, reflecting the fact that the Langevin equations (5) describe a Markovian process.

If there are no external forces present (i.e., $\mathcal{F}(t, x) \equiv 0$ ) then Eq. (15) yields the following FPE for the momentum PDF $\phi(t, p)$

$$
\frac{\partial \phi}{\partial t}=\frac{\partial}{\partial p}\left[\alpha(p) p \phi+D(p) \frac{\partial \phi}{\partial p}\right] .
$$

The stationary solution of Eq. (16) is given by

$$
\phi_{\infty}(p)=\mathcal{N} \exp \left[-\int_{-p_{*}}^{p} \mathrm{~d} p^{\prime} \frac{\alpha\left(p^{\prime}\right)}{D\left(p^{\prime}\right)} p^{\prime}\right],
$$

where $\mathcal{N}$ is a normalization constant, and $p_{*}$ some arbitrary constant such that the integral in the exponential exists.

The general form (17) of the stationary solution implies that one may generate arbitrary momentum distributions (e.g., Maxwell, Bose, Fermi or power law distributions) by choosing the friction and noise amplitude functions $\alpha(p)$ and $D(p)$ in a suitable manner $[19,425]$. To briefly illustrate this, consider some normalized target $\operatorname{PDF} \hat{\phi}(p) \geq 0$. We would like to fix the relation between $\alpha$ and $D$ such that the stationary solution $\phi_{\infty}(p)$ coincides with $\hat{\phi}(p)$. Equating $\hat{\phi}(p)$ with $\phi_{\infty}(p)$ from Eq. (17), taking the logarithm and differentiating with respect to $p$ we find the condition ${ }^{26}$

$$
\frac{\alpha(p)}{D(p)} p \equiv-\frac{\mathrm{d}}{\mathrm{d} p} \log \hat{\phi}(p)
$$

For instance, if a classical nonrelativistic Brownian particle is in thermal equilibrium with the surrounding heat bath, then $\hat{\phi}$ is a Maxwell distribution

$$
\hat{\phi}_{\mathrm{M}}(p)=(2 \pi M / \beta)^{-1 / 2} \exp \left[-\beta p^{2} /(2 M)\right], \quad \beta:=\left(k_{\mathrm{B}} \mathcal{T}\right)^{-1},
$$

where $\mathcal{T}$ is the temperature of the heat bath and $k_{\mathrm{B}}$ the Boltzmann constant. In this case, Eq. (18) reduces to the generalized Einstein fluctuation-dissipation relation $[25,112,113]$

$$
D(p)=\alpha(p) M k_{\mathrm{B}} \mathcal{T}=\alpha(p) M / \beta .
$$

The fluctuation-dissipation relations (18) and (20) do fix only one of the two coefficients $\alpha(p)$ and $D(p)$. Put differently, one is still free to adapt, for example, the function $\alpha(p)$ such that the stochastic process (5b) exhibits the correct relaxation behavior. This freedom is a main reason why the Langevin approach is successfully applicable to a wide range of thermalization processes [112]. Physically reasonable expressions for $\alpha(p)$ may be deduced from kinetic theory [315,316, 426-429] or microscopic Hamiltonian models that take into account the interactions as well as the statistical properties of the heat bath $[21,90-93,96,430]$. Examples will be discussed in Section 2.2 .

With regard to our subsequent discussion of relativistic Brownian motions, it will be important to keep in mind that the nonlinear Langevin equations like Eq. (5b) provide a tool for constructing Brownian motion processes with arbitrary stationary velocity and momentum distributions $[19,425]$.

\subsubsection{Nonrelativistic Ornstein-Uhlenbeck process}

The standard paradigm for a nonrelativistic Brownian motion process in the absence of external forces is the classical Ornstein-Uhlenbeck process [46], corresponding to constant coefficients

$$
D(P) \equiv D_{0}, \quad \alpha(p) \equiv \alpha_{0},
$$

yielding, e.g., the Ito SDE

$$
\begin{aligned}
& \mathrm{d} X=(P / M) \mathrm{d} t \\
& \mathrm{~d} P=\mathcal{F}(t, X) \mathrm{d} t-\alpha_{0} P \mathrm{~d} t+\left(2 D_{0}\right)^{1 / 2} * \mathrm{~d} B(t) .
\end{aligned}
$$

Note that in this particular case the choice of the discretization rule is not relevant when integrating the momentum equation (22b), but a rule must be specified when considering nonlinear transformations $G(P)$; cf. remarks in Appendix A.

\footnotetext{
25 If we had considered Eq. (5b) with another stochastic integral interpretation (e.g., pre-point or mid-point discretization), then the corresponding FPE would be different from Eq. (16)

26 If re-expressed in terms of the corresponding Ito or Stratonovich-Fisk friction coefficients $\alpha_{* \mid 0}(p)$ from Eqs. (10b) and (9b), then the derivative $D^{\prime}(p)$ enters the lhs. of Eq. (18), which thus would take the form of a differential equation.
} 
Free motion. Considering free Brownian motions with $\mathcal{F}(t, x) \equiv 0$ first, the solutions of Eqs. (5) read explicitly

$$
\begin{aligned}
& X(t)=X(0)+\int_{0}^{t} \mathrm{~d} s P(s) / M, \\
& P(t)=P(0) \mathrm{e}^{-\alpha_{0} t}+\left(2 D_{0}\right)^{1 / 2} \mathrm{e}^{-\alpha_{0} t} \int_{0}^{t} \mathrm{e}^{\alpha_{0} s} * \mathrm{~d} B(s) .
\end{aligned}
$$

Combining the solution (23) with Eq. (5d), one finds for the first two moments of the momentum coordinate [46,48]

$$
\begin{aligned}
& \langle P(t)\rangle=P(0) \mathrm{e}^{-\alpha_{0} t}, \\
& \left\langle P(t)^{2}\right\rangle=P(0)^{2} \mathrm{e}^{-2 \alpha_{0} t}+\frac{D_{0}}{\alpha_{0}}\left(1-\mathrm{e}^{-2 \alpha_{0} t}\right),
\end{aligned}
$$

while the first centered moments of the position coordinate are obtained as

$$
\begin{aligned}
& \langle X(t)-X(0)\rangle=\frac{P(0)}{\alpha_{0} M}\left(1-\mathrm{e}^{-\alpha_{0} t}\right), \\
& \left\langle[X(t)-X(0)]^{2}\right\rangle=\frac{2 D_{0}}{\left(\alpha_{0} M\right)^{2}} t+\left[\frac{P(0)}{\alpha_{0} M}\right]^{2}\left(1-\mathrm{e}^{-\alpha_{0} t}\right)^{2}+\frac{D_{0}}{\alpha_{0}^{3} M^{2}}\left(-3+4 e^{-\alpha_{0} t}-\mathrm{e}^{-2 \alpha_{0} t}\right) .
\end{aligned}
$$

The asymptotic spatial diffusion constant $\mathscr{D}_{\infty}$, not to be confused with the noise amplitude $D_{0}$, is usually defined by

$$
2 \mathscr{D}_{\infty}:=\lim _{t \rightarrow \infty} \frac{1}{t}\left\langle[X(t)-X(0)]^{2}\right\rangle .
$$

From Eq. (25b) we find for the Ornstein-Uhlenbeck process the classical result ${ }^{27}$

$$
D_{\infty}:=D_{0} /\left(\alpha_{0} M\right)^{2} \text {. }
$$

The FPE governing the momentum $\operatorname{PDF} \phi(t, p)$ of the free Ornstein-Uhlenbeck process reads [287]

$$
\frac{\partial \phi}{\partial t}=\frac{\partial}{\partial p}\left(\alpha_{0} p \phi+D_{0} \frac{\partial \phi}{\partial p}\right) .
$$

Adopting the deterministic initial condition (14b), $\phi(0, p)=\delta\left(p-p_{0}\right)$, the time-dependent solution of Eq. (28) is given by $[46,287]$

$$
\phi(t, p)=\left\{\frac{\alpha_{0}}{2 \pi D_{0}\left[1-\exp \left(-2 \alpha_{0} t\right)\right]}\right\}^{1 / 2} \exp \left\{-\frac{\alpha_{0}\left[p-p_{0} \exp \left(-\alpha_{0} t\right)\right]^{2}}{2 D_{0}\left[1-\exp \left(-2 \alpha_{0} t\right)\right]}\right\} .
$$

In the limit $t \rightarrow \infty$ this solution reduces to the stationary Gaussian distribution

$$
\phi_{\infty}(p)=\left(\frac{\alpha_{0}}{2 \pi D_{0}}\right)^{1 / 2} \exp \left(-\frac{\alpha p^{2}}{2 D_{0}}\right)
$$

For a given momentum distribution $\phi(t, p)$ of the Brownian particle, the corresponding velocity PDF $\psi(t, v)$ is defined by

$$
\psi(t, v):=\left|\frac{\mathrm{d} p}{\mathrm{~d} v}\right| \phi(t, p(v))
$$

where $p=M v$ in the nonrelativistic case. Hence, by imposing again the Einstein relation from Eq. (20), which now reduces to $D_{0}=\alpha_{0} M k_{\mathrm{B}} \mathcal{T}$, the stationary momentum PDF (30) is seen to be equivalent to Maxwell's velocity distribution

$$
\psi_{\mathrm{M}}(v)=\left(\frac{M}{2 \pi k_{\mathrm{B}} \mathcal{T}}\right)^{1 / 2} \exp \left(-\frac{M v^{2}}{2 k_{\mathrm{B}} \mathcal{T}}\right) .
$$

Moreover, by virtue of the Einstein relation $D_{0}=\alpha_{0} M k_{\mathrm{B}} \mathcal{T}$, the asymptotic diffusion constant from Eq. (27) can be written in the form

$$
D_{\infty}:=k_{\mathrm{B}} \mathcal{T} /\left(\alpha_{0} M\right) \text {. }
$$

27 A useful integral formula for the diffusion constant for nonlinear one-dimensional Brownian motion processes was derived by Lindner [25] recently, see Eq. (149). 
External force fields. The FPE describing the phase space density $f(t, x, p)$ of the stochastic process (22) in an external force field $\mathcal{F}(t, x)$ reads

$$
\frac{\partial f}{\partial t}+\frac{p}{M} \frac{\partial f}{\partial x}+\mathcal{F}(t, x) \frac{\partial f}{\partial p}=\frac{\partial}{\partial p}\left(\alpha_{0} p f+D_{0} \frac{\partial f}{\partial p}\right) .
$$

Models of this type have been intensely studied during the past century, covering a wide range of application (see, for example, Ref. [78] for a review). However, for arbitrary time and position dependent force fields $\mathcal{F}(t, x)$ it is generally very difficult, and in many cases even impossible, to find exact time-dependent solutions of the Fokker-Planck equation (34). In the simpler case of a time-independent, conservative force field $\mathcal{F}(t, x) \equiv F(x)$ with confining ${ }^{28}$ potential $\Phi(x)$, i.e.,

$$
F(x)=-\frac{\partial}{\partial x} \Phi(x),
$$

one can determine the stationary solution attained in the limit $t \rightarrow \infty$. Imposing, as above, the Einstein relation $D_{0}=$ $\alpha_{0} M k_{\mathrm{B}} \mathcal{T}$, the stationary solution of Eq. (34) is given by the Maxwell-Boltzmann distribution $[287,288]$

$$
f(x, p)=\mathcal{Z}^{-1} \exp \left\{-\beta\left[\frac{p^{2}}{2 M}+\Phi(x)\right]\right\}, \quad \beta:=\left(k_{\mathrm{B}} \mathcal{T}\right)^{-1},
$$

where the normalization constant $Z$ is determined by Eq. (12).

Another important class of applications concerns time periodic force fields, satisfying $\mathcal{F}(t, x)=\mathcal{F}(t+\Delta t, x)$ for some fixed period $\Delta t$. In this case it is sometimes possible to derive approximate asymptotic solutions of the FPE (34) by considering the limit $t \rightarrow \infty$. These asymptotic solutions are usually also time periodic and can exhibit phase shifts. They may give rise to a number of interesting phenomena such as, for example, stochastic resonance [431-441].

From the purely mathematical perspective, SDEs define well-defined models of stochastic processes [74]; from the physicist's point of view, their usefulness for the description of real systems is a priori an open issue. Hence, before directing our attention to the relativistic case, it is worthwhile to recall how nonrelativistic Langevin equations can be justified by means of microscopic models [21,90-96].

\subsection{Microscopic models}

When considering Langevin equations of the type (5), one may in principle distinguish between the two following tasks:

(a) One can postulate Langevin equations as phenomenological model equations, study the mathematical consequences and compare the resulting predictions with experiments in order to (in)validate the theory. Adopting this approach, the parameters and the explicit functional form of the friction and noise amplitude functions have to be determined from experimental data [442].

(b) Alternatively, one can try to motivate and derive Langevin equations from microscopic models. If successful, this approach yields explicit expressions for the friction and noise functions in terms of the microscopic model parameters.

The remainder of this section addresses the latter problem, which has attracted considerable interest over the past decades $[16,21,90-96,415,428,430,443]$. Langevin equations provide an approximate stochastic description of the 'exact' microscopic dynamics. Hence, in order to derive SDEs from, for example, Hamilton mechanics, one has to impose certain approximations. These approximations determine the range of applicability of the Langevin approach. Generally, one can pursue at least two different routes for deriving SDEs of the type (5) from more precise models:

(1) Starting from a Boltzmann-type equation [289,312,444] or master equation [75] for the one-particle probability density of the Brownian particle, one can try to reduce these integro-differential equations to a Fokker-Planck equation by performing suitable approximations [16,315,316,415,427-429,443,445-447]. Once the Fokker-Planck equation has been found, it is straightforward to write down a corresponding Langevin dynamics $[75,76]$. The microscopic collision dynamics is then encoded in the scattering cross-sections appearing in the collision integral of the Boltzmann equation $[312,444]$.

(2) Alternatively, one may start from a microscopic (e.g., Hamiltonian) model describing the interaction between Brownian particle and heat bath. After eliminating the heat bath degrees of freedom from the equations of motion for the Brownian particle [21,90-96,430], one obtains a generalized Langevin equation which may be reduced to the form (5) in certain limit cases. As a byproduct, the fluctuation-dissipation relations arise quite naturally within this approach upon assuming a probability distribution for the (initial) bath configuration.

To briefly illustrate the second procedure by example, we next consider the oscillator model [90-96] and the elastic binary collision model [21]. In contrast to the more frequently studied harmonic oscillator model, the collision model from Section 2.2.2 can be extended to the relativistic case; cf. discussion in Section 4.5.

\footnotetext{
28 Conventionally, a potential $\Phi(x)$ is called 'confining' if it increases sufficiently fast for $|x| \rightarrow \infty$ so that the phase space PDF $f$ is normalizable.
} 


\subsubsection{Harmonic oscillator model}

The harmonic oscillator model presents the classical paradigm for constructing a generalized Langevin equation from a Hamiltonian model [78,90-96]. The Hamiltonian function upon which the derivation is based reads [96]

$$
H=\frac{P^{2}}{2 M}+\Phi(X)+\sum_{r}\left[\frac{p_{r}^{2}}{2 m_{r}}+\frac{m_{r} \omega_{r}^{2}}{2}\left(x_{r}-\frac{c_{r}}{m_{r} \omega_{r}^{2}} X\right)^{2}\right] .
$$

Here $M, X$ and $P$ are the mass, position and momentum of the Brownian particle, and $\Phi(x)$ is an external potential field; $x_{r}$ and $p_{r}$ denote the position and momentum of a heat bath particle with mass $m_{r}$, oscillator frequency $\omega_{r}$ and coupling constant $c_{r}$. Eq. (37) yields the following Hamilton equations of motions:

$$
\begin{aligned}
& \dot{X}=(P / M), \quad \dot{P}=F(X)+\sum_{r} c_{r}\left(x_{r}-\frac{c_{r}}{m_{r} \omega_{r}^{2}} X\right) ; \\
& \dot{x}_{r}=\left(p_{r} / m_{r}\right), \quad \dot{p}_{r}=-m_{r} \omega_{r}^{2} x_{r}+c_{r} X, \quad r=1, \ldots, N,
\end{aligned}
$$

where $F(x)=-\mathrm{d} \Phi(x) / \mathrm{d} x$ is the conservative external force acting on the Brownian particle. As evident from Eq. (38), Brownian particle and heat bath are coupled via linear forces in this model. By formally integrating Eq. (38b) and inserting the solutions into Eq. (38a), one may eliminate the heat bath coordinates from Eq. (38a), yielding the exact generalized Langevin equations $[93,96]$

$$
\begin{aligned}
& \dot{X}=(P / M), \\
& \dot{P}=F(X)-\int_{0}^{t} \mathrm{~d} s v(t-s) P(s)+L(t),
\end{aligned}
$$

where, for given initial values $X(0), P(0),\left\{x_{r}(0), p_{r}(0)\right\}$, the memory friction kernel $v(t-s)$ and the Langevin noise force $L(t)$ are given by [96]

$$
\begin{aligned}
& v(t-s):=\frac{1}{M} \sum_{r} \frac{c_{r}^{2}}{m_{r} \omega_{r}^{2}} \cos \left[\omega_{r}(t-s)\right], \\
& L(t):=\sum_{r} c_{r}\left\{\left[x_{r}(0)-\frac{c_{r}}{m_{r} \omega_{r}^{2}} X(0)\right] \cos \left(\omega_{r} t\right)+\frac{p_{r}(0)}{m_{r} \omega_{r}} \sin \left(\omega_{r} t\right)\right\} .
\end{aligned}
$$

In order to be able to characterize the properties of the noise force $L(t)$ by means of a fluctuation-dissipation relation, one still needs to impose a distribution for the initial conditions $\left\{x_{r}(0), p_{r}(0)\right\}$ of the bath variables. In principle, this initial distribution can be chosen arbitrarily. Of particular interest in canonical thermostatistics are equilibrium distributions of the Maxwell-Boltzmann type. In the case of the generalized Langevin equation (39), a plausible choice for the initial bath distribution corresponds to the PDF

$$
f_{\mathrm{b}}\left(\left\{x_{r}(0), p_{r}(0)\right\} \mid X(0)=x_{0}\right)=\mathcal{Z}^{-1} \exp \left\{-\beta \sum_{r}\left[\frac{p_{r}(0)^{2}}{2 m_{r}}+\frac{m_{r} \omega_{r}^{2}}{2}\left(x_{r}(0)-\frac{c_{r}}{m_{r} \omega_{r}^{2}} x_{0}\right)^{2}\right]\right\},
$$

where $\beta=\left(k_{\mathrm{B}} \mathcal{T}\right)^{-1}$ is the inverse thermal energy, $\mathcal{T}$ the temperature, and $\mathcal{Z}$ the normalization constant. The initial position $X(0)=x_{0}$ of the Brownian particle enters in Eq. (40) as an independent parameter, i.e., averages with respect to $f_{\mathrm{b}}\left(\left\{x_{r}(0), p_{r}(0)\right\} \mid X(0)=x_{0}\right)$ are conditional on the initial Brownian particle position $X(0)=x_{0}$. Averaging the stochastic force $L(t)$ with respect to $f_{\mathrm{b}}$ from Eq. (40), one finds

$$
\begin{aligned}
& \langle L(t)\rangle_{\mathrm{b}}=0, \\
& \langle L(t) L(s)\rangle_{\mathrm{b}}=M k_{\mathrm{B}} \mathcal{T} v(t-s) .
\end{aligned}
$$

Eq. (41b) represents the fluctuation-dissipation relation for the generalized Langevin equation (39) given the initial bath distribution (40). The generalized Langevin equation (39) differs from Eqs. (5) and (22) through the memory friction $v(t-s)$. The SDE (22), describing the Ornstein-Uhlenbeck process in an external force field, is recovered from Eq. (39) in the limit case 29

$$
v(t-s)=2 \alpha_{0} \delta(t-s)
$$

where $\alpha$ is a constant friction coefficient. The limit case (42) can be illustrated by rewriting the friction kernel (39c) in the more general form

$$
v(t-s)=\int_{0}^{\infty} \mathrm{d} \omega C(\omega) \cos [\omega(t-s)] .
$$

29 The prefactor '2' is required in Eq. (42) because of the convention $\int_{0}^{t} \mathrm{~d} s \delta(t-s) P(s)=P(t) / 2$. 
By fixing the amplitude function $C(\omega)$ as

$$
C(\omega)=\frac{1}{M} \sum_{r} \frac{c_{r}^{2}}{m_{r} \omega_{r}^{2}} \delta\left(\omega-\omega_{r}\right)
$$

one recovers the memory friction (39c) as a special case of Eq. (43). In order to obtain the limit case (42) from Eq. (43), one can use the cosine-decomposition of the Dirac $\delta$-function

$$
\delta(t-s)=\frac{1}{2 \pi} \int_{-\infty}^{\infty} \mathrm{d} \omega \mathrm{e}^{\mathrm{i} \omega(t-s)}=\frac{1}{\pi} \int_{0}^{\infty} \mathrm{d} \omega \cos [\omega(t-s)] .
$$

Hence, upon comparing Eqs. (45) and (43), the white noise limit (42) corresponds to the particular choice

$$
C(\omega)=\left(2 \alpha_{0}\right) / \pi \text {. }
$$

The harmonic oscillator model ${ }^{30}$ provides a useful microscopic justification for the Langevin equations (5) and (22) of the Ornstein-Uhlenbeck process. Unfortunately, this model cannot be transferred to special relativity, as it is based on instantaneous harmonic interactions-at-distance which violate fundamental relativistic principles. Therefore, in the last part of this section we shall consider another microscopic model which is based on strictly localized elastic binary collisions and, thus, can be extended to special relativity.

\subsubsection{Elastic binary collision model}

The one-dimensional elastic binary collision model $[21,448]$ is based on the idea that the stochastic motion of a Brownian particle (mass $M$ ) is caused by frequent elastic collisions with smaller heat bath particles (mass $m \ll M$ ). ${ }^{31}$ As before, we denote the coordinates and momenta of the heat bath particles by $\left\{x_{r}, p_{r}\right\}$, where $r=1, \ldots, N$ and $N \gg 1$.

Collision kinematics. An elastic collision of the Brownian particle [velocity $V$, momentum $P=M V$, kinetic energy $E=$ $P^{2} /(2 M)$ ] with a heat bath particle [velocity $v_{r}$, momentum $p_{r}=m v_{r}$, kinetic energy $\epsilon_{r}=p_{r}^{2} /(2 m)$ ] is governed by the energy-momentum conservation laws

$$
E+\epsilon_{r}=\hat{E}+\hat{\epsilon}_{r}, \quad P+p_{r}=\hat{P}+\hat{p}_{r} .
$$

Here, hat-symbols refer to the state after the collision. Taking into account the kinematic conservation laws (47), we find that the momentum gain $\Delta P_{r}$ of the Brownian particle per single collision is given by

$$
\Delta P_{r}:=\hat{P}-P=-\frac{2 m}{M+m} P+\frac{2 M}{M+m} p_{r} .
$$

To construct a Langevin-like equation from Eqs. (47) and (48), one considers the total momentum change $\delta P(t)=P(t+$ $\delta t)-P(t)$ of the Brownian particle within the 'mesoscopic' time interval $[t, t+\delta t]$, assuming that:

- collisions occurring within $[t, t+\delta t]$ can be viewed as independent events;

- the time step $\delta t$ is sufficiently small, so that there occurs at most only one collision between the Brownian particle and a specific heat bath particle $r$ and that $|\delta P(t) / P(t)| \ll 1$ holds true;

- $\delta t$ is still sufficiently large, so that the total number of collisions within $\delta t$ is larger than 1.

These requirements can be fulfilled simultaneously only if $m \ll M$ holds. With the above assumptions, one can approximate

$$
\delta P(t) \approx \sum_{r=1}^{N} \Delta P_{r} I_{r}(t, \delta t)
$$

where $I_{r}(t, \delta t) \in\{0,1\}$ is the indicator function for a collision with the heat bath particle $r$ during the interval $[t, t+\delta t]$; i.e.

$$
I_{r}(t, \delta t)= \begin{cases}1 & \text { if a collision has occurred in }[t, t+\delta t] \\ 0 & \text { otherwise. }\end{cases}
$$

In the one-dimensional case, $I_{r}(t, \delta t)$ can be expressed in the form ${ }^{32}$

$$
I_{r}(t, \delta t)=\Theta\left(X-x_{r}\right) \Theta\left(x_{r}^{\prime}-X^{\prime}\right)+\Theta\left(x_{r}-X\right) \Theta\left(X^{\prime}-x_{r}^{\prime}\right)
$$

\footnotetext{
30 The quantum mechanical generalization of the harmonic oscillator model represents a paradigm for quantum Brownian motions and has been studied, for example, in [92,97-103,109]; see also the reviews by Grabert et al. [118] and Hänggi and Ingold [108].

31 Similar approaches are known from unimolecular rate theory - see, for example, Section V in [78]. In the context of quantum Brownian motions, a quantum-mechanical version of the collision model was proposed and studied by Pechukas [105], and Tsonchev and Pechukas [106].

32 The Heaviside-function $\Theta(x)$ is defined as the integral over the Dirac $\delta$-function, i.e., $\Theta(x):=0, x<0 ; \Theta(0):=1 / 2 ; \Theta(x):=1, x>0$. When considering higher space dimensions, the expression (51) for the indicator function has to be modified accordingly - e.g., by taking into account the geometric shape of the Brownian particle.
} 
where $X=X(t), x_{r}=x_{r}(t)$ are the 'initial' positions of the colliding particles at time $t$, and

$$
X^{\prime}=X+V \delta t, \quad x_{r}^{\prime}=x_{r}+v_{r} \delta t
$$

their projected positions ${ }^{33}$ at time $t+\delta t$. The collision indicator from Eq. (51) is characterized by

$$
I_{r}(t, 0)=0, \quad\left[I_{r}(t, \delta t)\right]^{j}=I_{r}(t, \delta t), \quad j=1,2, \ldots
$$

The Taylor-expansion of $I_{r}$ at $\delta t=0$ reads [21]

$$
I_{r}(t, \delta t) \approx \frac{\delta t}{2}\left|v_{r}-V\right| \delta\left(x_{r}-X\right) .
$$

Combining Eqs. (48), (49) and (53a) yields

$$
\delta P(t) \approx-2\left[\sum_{r=1}^{N} \frac{m}{M+m} I_{r}(t, \delta t)\right] P(t)+2 \sum_{r=1}^{N} \frac{M}{M+m} p_{r} I_{r}(t, \delta t),
$$

where, additionally, it was assumed that for each collision occurring within $[t, t+\delta t]$, the momentum of the Brownian particle before the collision is approximately equal to the 'initial' value $P(t)$. In view of $m \ll M$, Eq. (53b) can be simplified further to give

$$
\delta P(t) \approx-2\left[\sum_{r=1}^{N} \frac{m}{M} I_{r}(t, \delta t)\right] P(t)+2 \sum_{r=1}^{N} p_{r} I_{r}(t, \delta t) .
$$

A comparison with the Langevin equation (5b) suggests that, heuristically, the first term on the rhs of Eq. (53c) can be interpreted as a 'friction' term, while the second term represents 'noise'. However, although looking quite similar to a Langevin equation, Eq. (53c) is still considerably more complicated than, for example, the Langevin equation (5b). This is due to the fact that the collision indicators $I_{r}(t, \delta t)$ from Eq. (53a) depend not only on the Brownian particle's position and velocity but also on the stochastic bath variables $\left\{x_{r}, v_{r}\right\}$. Nevertheless, it is possible to calculate the statistical properties of the momentum increments $\delta P(t)$ from Eq. (53), provided one specifies a distribution for the heat bath particles.

Bath distribution. In principle, one can use Eq. (53) to calculate the statistical moments $\left\langle(\delta P)^{j}\right\rangle_{\mathrm{b}}$ for an arbitrarily given heat bath $\operatorname{PDF} f_{\mathrm{b}}^{N}\left(\left\{x_{r}, p_{r}\right\}\right)$. Here, we shall focus on the situation where the (infinitely large) heat bath is given by a quasi-ideal gas which is in thermal equilibrium with its environment. In this case, the one-particle $\operatorname{PDF} f_{\mathrm{b}}^{1}\left(x_{r}, p_{r}\right)$ is given by the spatially homogeneous Maxwell distribution

$$
f_{\mathrm{b}}^{1}\left(x_{r}, p_{r}\right)=\left(2 \pi m k_{\mathrm{B}} \mathcal{T}\right)^{-1 / 2} L^{-1} \exp \left[-p_{r}^{2} /\left(2 m k_{\mathrm{B}} \mathcal{T}\right)\right],
$$

where $x_{r} \in[0, L]$ with $L$ being the one-dimensional container volume. Moreover, we will assume that:

- the heat bath particles are independently and identically distributed;

- the distribution of the bath particles is not affected by the collisions with the Brownian particle.

The above assumptions can be justified for a sufficiently large bath, if collisions between the bath particles rapidly reestablish a spatially homogeneous bath distribution.

Mean drift force. We define the mean (momentum) drift as the average momentum change $\langle\delta P\rangle_{\mathrm{b}}$ over the interval $[t, t+\delta t]$, given the momentum value $P$ at time $t .^{34}$ In the case of Eq. (53c), one finds $[21,448]$

$$
\langle\delta P(t)\rangle_{\mathrm{b}}=-2 N\left(\frac{m}{M}\right)\left\langle I_{r}(t, \delta t)\right\rangle_{\mathrm{b}} P+2 N\left\langle p_{r} I_{r}(t, \delta t)\right\rangle_{\mathrm{b}} .
$$

To calculate the averages on the rhs we note that, for a spatially uniform bath distribution, as in Eq. (54), one-particle expectation values of the form $\left\langle G\left(x_{r}, v_{r}\right) I_{r}(t, \delta t)\right\rangle_{\mathrm{b}}$ can be calculated to first order in $\delta t$ as [21]

$$
\left\langle G\left(x_{r}, v_{r}\right) I_{r}(t, \delta t)\right\rangle_{\mathrm{b}}=\frac{\delta t}{2 L} \int_{-\infty}^{\infty} \mathrm{d} v_{r} G\left(X, v_{r}\right)\left|v_{r}-V\right| \psi_{\mathrm{b}}\left(v_{r}\right) .
$$

Here, $\psi_{\mathrm{b}}\left(v_{r}\right)$ denotes the one-particle velocity PDF of the heat bath particles which, in the case of Eq. (54), is given by the Maxwellian

$$
\psi_{\mathrm{b}}\left(v_{r}\right)=\left(v_{\mathrm{B}}^{2} \pi\right)^{-1 / 2} \exp \left(-v_{r}^{2} / v_{\mathrm{B}}^{2}\right), \quad v_{\mathrm{B}}:=\left(2 k_{\mathrm{B}} \mathcal{T} / m\right)^{1 / 2} .
$$

\footnotetext{
33 Of course, in the case of a collision the position of the particles at time $t+\delta t$ will be different from the 'projected' positions $X^{\prime}$ and $x^{\prime}$.

34 More precisely, one should write the mean momentum drift in the form of a conditional expectation $\langle\delta P(t) \mid P(t)=p\rangle_{\mathrm{b}}$; however, for ease of notation we write $\langle\delta P\rangle_{\mathrm{b}}$ at this stage.
} 


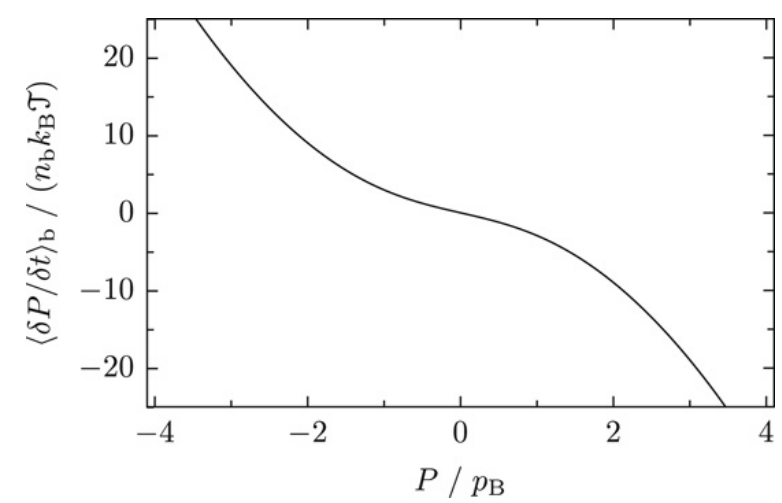

Fig. 1. Nonrelativistic binary collision model. Mean drift force $\mathcal{K}(P)=\langle\delta P(t) / \delta t\rangle_{\mathrm{b}}$ from Eq. (58) with $n_{\mathrm{b}}=N / L$ denoting the number density of the heat bath particles and $p_{\mathrm{B}}:=M\left(2 k_{\mathrm{B}} \mathcal{T} / m\right)^{1 / 2}$ the characteristic momentum of a Brownian particle (mass $M$ ), surrounded by heat bath particles of mass $m$ and temperature $\mathcal{T}$.

By making use of Eq. (56), one obtains for the mean drift of the collision model: ${ }^{35}$

$$
\langle\delta P(t)\rangle_{\mathrm{b}} \approx-2 n_{\mathrm{b}} k_{\mathrm{B}} \mathcal{T}\left\{\pi^{-1 / 2}\left(\frac{P}{p_{\mathrm{B}}}\right) \exp \left[-\left(\frac{P}{p_{\mathrm{B}}}\right)^{2}\right]+\left[\left(\frac{P}{p_{\mathrm{B}}}\right)^{2}+\frac{1}{2}\right] \operatorname{erf}\left(\frac{P}{p_{\mathrm{B}}}\right)\right\} \delta t,
$$

where $n_{\mathrm{b}}=N / L$ is the number density of the heat bath particles, $p_{\mathrm{B}}:=M v_{\mathrm{B}}=M\left(2 k_{\mathrm{B}} \mathcal{T} / m\right)^{1 / 2}$ a characteristic thermal momentum scale, and the error function $\operatorname{erf}(z)$ is defined by

$$
\operatorname{erf}(z):=\frac{2}{\sqrt{\pi}} \int_{0}^{z} \mathrm{~d} x \mathrm{e}^{-x^{2}}
$$

Fig. 1 depicts the mean drift force

$$
\mathcal{K}(P):=\langle\delta P(t) / \delta t\rangle_{\mathrm{b}},
$$

obtained from Eq. (58). The absolute value of this drift force grows linearly for small momentum values (Ornstein-Uhlenbeck regime) and quadratically for large momentum values.

In the remainder of this section, we are going to illustrate how one can use the result (58) to obtain a systematic procedure for approximating the model equations (53) by a nonlinear SDE of the type (5b).

Langevin approximation. Similar to the oscillator equation (39), the Eq. (53c) for the momentum increments $\delta P(t)$ in the binary collision model is not yet a Langevin equation. SDEs of the type

$$
\mathrm{d} P(t)=-\alpha(P) P \mathrm{~d} t+[2 D(P)]^{1 / 2} \bullet \mathrm{d} B(t)
$$

are phenomenological model equations that provide a simplified description of the microscopic dynamics, which in the case of the collision model is more precisely described by Eq. (53c). Therefore, to obtain a useful Langevin model, the coefficients $\alpha(p)$ and $D(p)$ have to be chosen such that they yield the best possible approximation within this class of SDEs defined by Eq. (60). Plausible general criteria for the 'best approximation' can be formulated as follows:

The stochastic process described by Eq. (60) should

- approach the correct stationary momentum distribution;

- exhibit the correct mean relaxation behavior.

The first criterion is equivalent to imposing the appropriate fluctuation-dissipation relation on the functions $\alpha$ and $D$. For the elastic collision model considered here, the expected stationary momentum PDF of the Brownian particle is given by the Maxwell distribution

$$
\phi_{\infty}(p)=\left(2 \pi M k_{\mathrm{B}} \mathcal{T}\right)^{-1 / 2} \exp \left[-p^{2} /\left(2 M k_{\mathrm{B}} \mathcal{T}\right)\right]
$$

According to the discussion in Section 2.1.1, this implies that $\alpha$ and $D$ must be coupled by the Einstein condition

$$
D(P)=\alpha(P) M k_{\mathrm{B}} \mathcal{T} .
$$

The second (drift) criterion can be expressed mathematically as ${ }^{36}$

$$
\left\langle\frac{\mathrm{d} P(t)}{\mathrm{d} t} \mid P(t)=p\right\rangle \stackrel{!}{=}\left\langle\frac{\delta P(t)}{\delta t} \mid P(t)=p\right\rangle_{\mathrm{b}} .
$$

\footnotetext{
35 Higher moments and correlation functions may be calculated in a similar manner.

$36\langle\cdot \mid P(t)=p\rangle$ denotes by the conditional expectation with respect to the Wiener measure of the Brownian motion $B(t)$.
} 

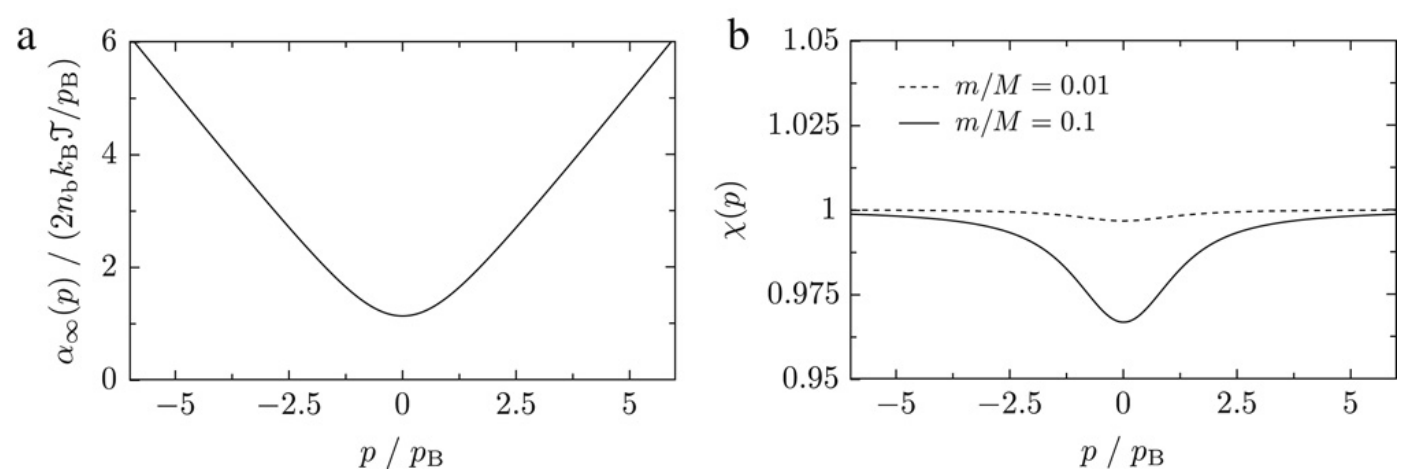

Fig. 2. Nonrelativistic binary collision model. (a) Friction coefficient $\alpha_{\infty}(p)=-\mathcal{K}(p) / p$ from Eq. (66) with $n_{\mathrm{b}}=N / L$ denoting the number density of the heat bath particles and $p_{\mathrm{B}}:=M\left(2 k_{\mathrm{B}} \mathcal{T} / m\right)^{1 / 2}$ the characteristic momentum of a Brownian particle (mass $\left.M\right)$, surrounded by heat bath particles of mass $m$ and temperature $\mathcal{T}$. (b) The ratio $\chi(p)$ from Eq. (67) as a measure for the quality of the approximation $\alpha_{\infty}(p)$.

Taking into account the Einstein relation and Eq. (11a), the lhs. of Eq. (63) is given by

$$
\left\langle\frac{\mathrm{d} P(t)}{\mathrm{d} t} \mid P(t)=p\right\rangle=-\left[\alpha(p) p-\alpha^{\prime}(p) M k_{\mathrm{B}} \mathcal{T}\right],
$$

where $\alpha^{\prime}(p):=\mathrm{d} \alpha(p) / \mathrm{d} p$. The rhs of Eq. (63) is obtained by substituting $P=p$ on the rhs of Eq. (58), yielding, i.e.,

$$
\left\langle\frac{\delta P(t)}{\delta t} \mid P(t)=p\right\rangle_{\mathrm{b}}=\mathcal{K}(p),
$$

where the mean drift force $\mathcal{K}$ was defined in Eq. (59). Hence, by virtue of Eq. (64), we see that the drift criterion (63) is equivalent to the following ordinary differential equation (ODE) for $\alpha(p)$ :

$$
-\alpha(p) p+\alpha^{\prime}(p) M k_{\mathrm{B}} \mathcal{T}=\mathcal{K}(p) .
$$

With respect to the two criteria formulated above, the solution of this ODE gives the friction function $\alpha$ that provides the 'best' Langevin approximation to the binary collision model. The initial condition for $\alpha(p)$ must be specified such that the correct asymptotic behavior is obtained [448]. Information about the collision model and the bath distribution is encoded in the mean drift force $\mathcal{K}(p)$. Evidently, the procedure leading to Eq. (65) can be generalized to other interaction models/bath distributions as well - provided the stationary distribution of the Brownian particle is known. Other types of interactions (e.g., nonelastic) would result in another function $\mathcal{K}(p)$. A non-Maxwellian bath distribution would affect not only the rhs of Eq. (65) but also its lhs. due to a modified fluctuation-dissipation relation.

Unfortunately, it is usually very difficult or even impossible to find the exact analytical solution of the ODE (65) for a realistic drift function $\mathcal{K}(p)$. For practical purposes, one can obtain useful approximations, for example, by considering the asymptotic behavior for $p \rightarrow \infty$ and $p \rightarrow 0$, respectively. In the case of the collision model, one finds that the approximation

$$
\alpha(p) \simeq-\mathcal{K}(p) / p=: \alpha_{\infty}(p),
$$

which becomes exact for $p \rightarrow \infty$, is also applicable at small $|p|$-values, if $m \ll M$. This is illustrated in Fig. 2(b), which depicts the dimensionless ratio

$$
\chi(p):=\left[-\alpha_{\infty}(p) p+\alpha_{\infty}^{\prime}(p) M k_{\mathrm{B}} \mathcal{T}\right] / \mathcal{K}(p) .
$$

The function $\chi$ is an indicator for the quality of the approximative solution $\alpha_{\infty}(p)$, which is plotted in Fig. 2(a). As evident from the dotted curve in Fig. 2(b), for $m \ll M$ the function $\chi$ is close to unity even for small values $|p|$. This means that $\alpha_{\infty}$ is a good approximation to the exact solution of Eq. (65). Thus, a Langevin equation of the type (60) with $\alpha_{\infty}(p)$ from Eq. (66) and $D(p)=\alpha_{\infty}(p) M k_{\mathrm{B}} \mathcal{T}$ yields the correct stationary momentum distribution (61) and exhibits almost exactly the same mean relaxation behavior as Eq. (53). In particular, such a nonlinear Langevin equation provides a considerably more accurate description of the Brownian motion in a gaseous heat bath than, for example, a classical Ornstein-Uhlenbeck process with constant friction and noise coefficients, cf. Eq. (22b). For instance, an Ornstein-Uhlenbeck (or Stokes-like) approximation is obtained by replacing $\alpha_{\infty}(p)$ through its minimum value

$$
\alpha_{0}:=\alpha_{\infty}(0)=-\mathcal{K}^{\prime}(0)=n_{\mathrm{b}} \frac{m}{M}\left(\frac{8 k_{\mathrm{B}} \mathcal{T}}{\pi m}\right)^{1 / 2}
$$

and fixing the Einstein relation $D(p)=\alpha_{0} M k_{\mathrm{B}} \mathcal{T}=: D_{0}$. Adopting these additional simplifications, the corresponding Ornstein-Uhlenbeck process can be expected to provide a useful description for slow Brownian particles at sufficiently low temperature values $\mathcal{T}$. 
Similar to the harmonic oscillator model from Section 2.2.1, the collision model provides useful insights into the approximations that must be made in order to obtain a Langevin equation from a microscopic model. Compared with the oscillator model, an advantage of the collision model is given by the fact that it can be extended to special relativity, cf. Section 4.5.

From a more general perspective, the above examples illustrate which objectives can(not) be achieved by phenomenological Langevin models that are based on Brownian motion processes. Langevin equations of this type, and their corresponding Fokker-Planck equations, provide a simplified description of the underlying microscopic dynamics. The coefficient functions in the Langevin/Fokker-Planck equations allow one to construct stochastic processes that exhibit the same asymptotic relaxation behavior and approach the same stationary or asymptotic distribution as the actual physical process. Stationary distributions can often be inferred from thermostatistical (e.g., maximum entropy) principles, while the relaxation behavior must be deduced from the exact microscopic dynamics. In many cases, the resulting stochastic models are sufficient for comparing with experimentally accessible data, but they may become inaccurate for describing higher order correlations and/or the relaxation dynamics far from the asymptotic state.

\subsection{Remarks and generalizations}

In this section we have focused on the most commonly considered examples of nonrelativistic Brownian motion processes, described by SDEs of the type (5). Such nonrelativistic Langevin equations have been studied extensively in various contexts over the past decades (see, e.g., the reviews [78,112]). The list of successful applications covers a wide range of different areas including laser physics [112,113,449], optical lattices [450,451], plasma physics [452-455], high energy physics [315,316], biologically and chemically motivated population and reaction dynamics [456], active Brownian motion models [429,457-462], or theoretical and experimental studies of excitation and transition phenomena in nonlinear systems [463-466].

The stochastic processes defined by Eq. (5) share, as a common feature, that the underlying noise source is modeled by a standard Wiener process $B(t)$. In general, one can also consider other driving processes such as Poisson processes $[74,76]$ or Lévy processes [467-471], which may give rise to so-called anomalous super- or sub-diffusion effects; see, e.g., the reviews by Bouchaud and Georges [116] and Metzler and Klafter [117]. ${ }^{37}$ Furthermore, one can abandon the assumption (4b) of $\delta$-correlated 'white' noise by considering stochastic processes that are driven by 'colored' noise, for example, by replacing Eq. (4b) with an exponential correlation

$$
\langle\zeta(t) \zeta(s)\rangle=\frac{1}{\tau_{\mathrm{n}}} \exp \left(-|t-s| / \tau_{\mathrm{n}}\right),
$$

where the parameter $\tau_{\mathrm{n}}$ is the relaxation time of the driving noise $\zeta$. The mathematical analysis of processes driven by colored noise is considerably more complicated than in the case of $\delta$-correlated white noise (see Ref. [114] for a review). The study of non-Gaussian and/or colored driving processes within a relativistic framework still represents an open problem for the future. By contrast, the relativistic generalization of stochastic processes of the type (5) has been the subject of intense research in recent years, leading to the relativistic Brownian motion theory described in Section 4.

\section{Relativistic equilibrium thermostatistics}

The brief summary of nonrelativistic Brownian motion theory in the preceding section shows that equilibrium thermostatistics plays an important role in constraining the relation between friction and noise coefficients in Langevin equations by means of suitably chosen fluctuation-dissipation relations. Moreover, a priori knowledge about the equilibrium properties of the heat bath is required if one wishes to derive (generalized) Langevin equations from microscopic models. Therefore, the present section intends to summarize relevant aspects of relativistic equilibrium thermostatistics in order to provide for the subsequent discussion of relativistic Langevin equations in Section 4. This part is structured as follows.

Section 3.1 introduces some notation and discusses general properties of relativistic probability density functions. Section 3.2 focuses on the thermostatistics of stationary systems, since these will play the role of a heat bath later on. In this context, particular emphasis will be placed on the relativistic generalization of Maxwell's distribution for the following reason: Nonrelativistic Brownian motion models such as the classical Ornstein-Uhlenbeck process are in obvious conflict with special relativity because they permit particles to move faster than the speed of light $c$. Most directly, this can be seen from the stationary velocity distribution, which is a Maxwell velocity distribution and thus non-zero for velocities $|\boldsymbol{v}|>c$. The recent literature has seen some debate about the correct generalization of Maxwell's distribution in special relativity $[22,196,198,199,232,475]$. In Section 3.2 we shall discuss recent molecular dynamics simulations [202] which favor a distribution that was proposed by Jüttner [186] in 1911, i.e., six years after Einstein had formulated his theory of special relativity [2,3]. In addition to its relevance with regard to relativistic Brownian motion theory, the Jüttner gas also

\footnotetext{
37 Anomalous diffusion processes [472-474] exhibit a nonlinear growth of the mean square displacement, for example, of the form to $t^{\kappa}$ with $\kappa>1$ and $\kappa<1$ corresponding super- and sub-diffusion, respectively [117,467].
} 
provides a useful model for illustrating the subtleties of relativistic thermodynamics. The latter topic is discussed separately in Appendix C, also addressing the controversy that has surrounded the Lorentz transformation laws of temperature and other thermodynamic quantities over the past 100 years $[121,122,127-149,156,158-160,165,166,169,171,172,178,214,215$, $217,234,476]$.

\subsection{Preliminaries}

Section 3.1.1 summarizes definitions and notational conventions. ${ }^{38}$ Subsequently, the 'peculiar' Lorentz transformation behavior of one-particle phase space probability density functions (PDFs) will be addressed.

\subsubsection{Notation and conventions}

In special relativity, an inertial frame $\Sigma$ corresponds to a global Cartesian spacetime coordinate system. A spacetime event $\mathscr{E}$ is labeled by a $(1+d)$-dimensional coordinate tuple $\bar{x}=\left(x^{\alpha}\right)=(c t, \boldsymbol{x})=\left(t, x^{1}, \ldots, x^{d}\right)$ in $\Sigma$, where $d$ is the number of space dimensions and, adopting natural units from now on, the speed of light $c=1$. Upper and lower Greek indices $\alpha, \beta, \ldots$ take values $0,1, \ldots, d$, and we use Latin indices $i, k, \ldots \in\{1, \ldots, d\}$ for the spatial components. Vectors with upper indices are called contravariant.

With respect to the Cartesian coordinate frame $\Sigma$, the components $\eta_{\alpha \beta}$ of the metric tensor of flat Minkowski spacetime are defined by [6]

$$
\eta_{\alpha \beta}= \begin{cases}-1 & \alpha=\beta=0 \\ +1 & \alpha=\beta=1, \ldots, d \\ 0 & \alpha \neq \beta .\end{cases}
$$

By definition, the components of the covariant vector $\left(x_{\alpha}\right)$ are obtained by contracting the contravariant vector $\left(x^{\alpha}\right)$ with $\eta_{\alpha \beta}$, i.e., ${ }^{39}$

$$
x_{\alpha}:=\sum_{\beta=0}^{d} \eta_{\alpha \beta} x^{\beta}=: \eta_{\alpha \beta} x^{\beta} \Rightarrow\left(x_{\alpha}\right)=(-t, \boldsymbol{x}) .
$$

The tuples $\left(x^{\alpha}\right)$ and $\left(x_{\alpha}\right)$ will be called four-vectors, regardless of the number of space dimensions. The corresponding fourvector gradients are defined by

$$
\begin{aligned}
\left(\partial_{\alpha}\right) & :=\left(\frac{\partial}{\partial x^{\alpha}}\right)=\left(\frac{\partial}{\partial t}, \frac{\partial}{\partial x^{1}}, \ldots, \frac{\partial}{\partial x^{d}}\right)=\left(\frac{\partial}{\partial t}, \nabla\right), \\
\left(\partial^{\alpha}\right) & :=\left(\frac{\partial}{\partial x_{\alpha}}\right)=\left(-\frac{\partial}{\partial t}, \frac{\partial}{\partial x^{1}}, \ldots, \frac{\partial}{\partial x^{d}}\right)=\left(-\frac{\partial}{\partial t}, \nabla\right) .
\end{aligned}
$$

The components $\eta^{\alpha \beta}$ of the inverse metric tensor are determined by the condition

$$
x^{\alpha} \stackrel{!}{=} \eta^{\alpha \beta} x_{\beta}=\eta^{\alpha \beta} \eta_{\beta \gamma} x^{\gamma} \quad \forall\left(x^{\alpha}\right),
$$

or, equivalently, by

$$
\eta^{\alpha \beta} \eta_{\beta \gamma} \stackrel{!}{=} \delta^{\alpha}{ }_{\gamma}
$$

where $\delta^{\alpha}{ }_{\gamma}$ is the Kronecker $\delta$-symbol, yielding

$$
\eta^{\alpha \beta}=\eta_{\alpha \beta}
$$

The Minkowski spacetime distance between two events $\bar{x}_{A}=\left(x_{A}^{\alpha}\right)=\left(t_{A}, \boldsymbol{x}_{A}\right)$ and $\bar{x}_{B}=\left(x_{B}^{\alpha}\right)=\left(t_{B}, \boldsymbol{x}_{B}\right)$ is defined by

$$
\begin{aligned}
d\left(\bar{x}_{A}, \bar{x}_{B}\right)^{2} & :=\eta_{\alpha \beta}\left(x_{A}^{\alpha}-x_{B}^{\alpha}\right)\left(x_{A}^{\beta}-x_{B}^{\beta}\right) \\
& =-\left(t_{A}-t_{B}\right)^{2}+\left(\boldsymbol{x}_{A}-\boldsymbol{x}_{B}\right)^{2} .
\end{aligned}
$$

By definition, the separation of two events is

- time-like, if $d\left(\bar{x}_{A}, \bar{x}_{B}\right)^{2}<0$;

- light-like, if $d\left(\bar{x}_{A}, \bar{x}_{B}\right)^{2}=0$;

- space-like, if $d\left(\bar{x}_{A}, \bar{x}_{B}\right)^{2}>0$.

38 For a more detailed introduction to special relativity we refer to Weinberg [6] or Sexl and Urbantke [8]

39 Throughout, we will use the Einstein summation convention defined in Eq. (71). 
Events with time-like separation can be causally connected by (a series of) signals travelling slower or equal to the speed of light. Events with light-like separation can be causally related only by undisturbed signals travelling at the speed of light. Events with space-like separation are causally disconnected.

The classical motion of a massive point-like particle through spacetime corresponds to a sufficiently smooth, time-like curve in $\Sigma$, referred to as world-line. Consider a stationary observer $\mathcal{O}$, who is at rest in $\Sigma$. It is natural that $\mathcal{O}$ parameterizes the particle motion using the coordinate time $t$ of $\Sigma$, i.e., $\mathcal{O}$ describes the world-line as a curve $\left(x^{0}(t), x^{i}(t)\right)$ with $x^{0}(t)=t$. In the vicinity of any point (event) on the particle's world-line, an infinitesimal proper time differential can be defined by

$$
\mathrm{d} \tau:=\left(-\eta_{\alpha \beta} \mathrm{d} x^{\alpha} \mathrm{d} x^{\beta}\right)^{1 / 2}=\left(\mathrm{d} t^{2}-\mathrm{d} \boldsymbol{x}^{2}\right)^{1 / 2}=\mathrm{d} t\left(1-\boldsymbol{v}^{2}\right)^{1 / 2}
$$

where $\boldsymbol{v}(t):=\mathrm{d} \boldsymbol{x}(t) / \mathrm{d} t$ is the particle velocity in $\Sigma$. According to the postulates of special relativity, $\mathrm{d} \tau$ is the time interval measured by an intrinsic clock, comoving with the particle, whereas $\mathrm{d} t$ is the coordinate time interval measured by a clock at rest in $\Sigma$. The four-velocity $\left(u^{\alpha}\right)$ of a massive particle is defined as the derivative of the world-line with respect to its proper time,

$$
u^{\alpha}:=\frac{\mathrm{d} x^{\alpha}}{\mathrm{d} \tau} \Rightarrow u_{\alpha} u^{\alpha}=-1
$$

For a point-like particle with rest mass $m>0$, the energy-momentum four-vector $\left(p^{\alpha}\right)=\left(p^{0}, p^{1}, \ldots, p^{d}\right)=(\epsilon, \boldsymbol{p})$ is defined by

$$
p^{\alpha}:=m u^{\alpha} \Rightarrow p_{\alpha} p^{\alpha}=-m^{2}
$$

Upon comparing with (76), one finds for a particle with velocity $\boldsymbol{v}$ in $\Sigma$

$$
p^{0}=\epsilon=m \gamma(\boldsymbol{v}), \quad \boldsymbol{p}=m \gamma(\boldsymbol{v}) \boldsymbol{v}, \quad \gamma(\boldsymbol{v}):=\left(1-\boldsymbol{v}^{2}\right)^{-1 / 2} .
$$

Lorentz-Poincaré transformations. In special relativity affine-linear Lorentz-Poincaré transformations (LPTs) of the form

$$
\bar{x}^{\prime}=\Lambda \bar{x}+\bar{a} \quad \Leftrightarrow \quad x^{\prime \alpha}=\Lambda_{\beta}^{\alpha} x^{\beta}+a^{\alpha}
$$

describe the transition from an inertial frame $\Sigma$ to another inertial frame $\Sigma^{\prime}$. The constant four-vector $a^{\alpha}$ shifts the origins of time and space, while the constant Lorentz matrix $\left(\Lambda_{\beta}^{\alpha}\right)$ may account for a spatial rotation, a change of orientation and/or a relative velocity between the two frames $\Sigma$ and $\Sigma^{\prime}$ [6]. The matrix components $\Lambda^{\alpha}{ }_{\beta}$ are determined by the condition

$$
d\left(\bar{x}_{A}^{\prime}, \bar{x}_{B}^{\prime}\right)^{2} \stackrel{!}{=} d\left(\bar{x}_{A}, \bar{x}_{B}\right)^{2} \Leftrightarrow \Lambda_{\gamma}^{\alpha} \Lambda_{\delta}^{\beta} \eta_{\alpha \beta} \stackrel{!}{=} \eta_{\gamma \delta},
$$

which means that causal relations remain preserved during transitions between inertial systems. The LPTs (80) form a group. ${ }^{40}$ Of particular interest for our purpose, is the subgroup of proper Lorentz transformations (LTs), defined by $a^{\alpha}=0$ and the additional constraints

$$
\Lambda_{0}^{0} \geq 1, \quad \operatorname{det}\left(\Lambda_{\beta}^{\alpha}\right)=+1 .
$$

The requirements (81) exclude time reversal and space inversion. Examples are pure rotations

$$
\Lambda_{0}^{0}=1, \quad \Lambda_{0}^{i}=\Lambda^{0}{ }_{i}=0, \quad \Lambda_{j}^{i}=R_{i j},
$$

where $\left(R_{i j}\right)$ is a rotation matrix [i.e., $\operatorname{det}\left(R_{i j}\right)=1$ and $R_{i j} R_{k j}=\delta_{i j}$ ], and Lorentz boosts [6]

$$
\Lambda_{0}^{0}=\gamma, \quad \Lambda_{0}^{i}=\Lambda^{0}{ }_{i}=-\gamma w^{i}, \quad \Lambda_{j}^{i}=\delta_{j}^{i}+\frac{w^{i} w^{j}}{\boldsymbol{w}^{2}}(\gamma-1)
$$

with velocity $\boldsymbol{w}=\left(w^{1}, \ldots, w^{d}\right)$ and Lorentz factor $\gamma:=\left(1-\boldsymbol{w}^{2}\right)^{-1 / 2}$. To briefly illustrate the effect of a boost, consider a particle at rest in the spatial origin of $\Sigma$ and, therefore, being described by the world-line $\left(x^{\alpha}(t)\right) \equiv(t, \boldsymbol{0})$ in $\Sigma$. By applying the Lorentz boost $(83)$ to $\left(x^{\alpha}\right)=(t, \mathbf{0})$, we find

$$
x^{\prime 0}=\Lambda_{0}^{0} x^{0}=\gamma t=t^{\prime}, \quad x^{i}=\Lambda_{0}^{i} x^{0}=-\gamma w^{i} t=-w^{i} t^{\prime},
$$

which means that the particle travels at constant velocity $\boldsymbol{w}^{\prime}=-\boldsymbol{w}$ through $\Sigma^{\prime}$; i.e., $\Sigma^{\prime}$ moves with velocity $\boldsymbol{w}$ relative to $\Sigma$. The inverse of the transformation matrix Eq. (83) is obtained by replacing $\boldsymbol{w}$ with $-\boldsymbol{w}$.

From Eq. (80) and the definition (78) of the four-momentum, one finds the relativistic energy-momentum transformation law

$$
p^{\prime \alpha}=\Lambda_{\beta}^{\alpha} p^{\beta} \text {. }
$$

Combining Eqs. (85) and (80b), one can verify the well-known mass-shell condition

$$
m^{2}=\epsilon^{2}-\boldsymbol{p}^{2}=\epsilon^{\prime 2}-\boldsymbol{p}^{2}=m^{\prime 2}
$$

which means that the rest mass $m$ is a Lorentz invariant. In particular, the mass shell condition (86) implies that Eq. (85) is equivalent to a restricted nonlinear transformation $\boldsymbol{p} \mapsto \boldsymbol{p}^{\prime}=\boldsymbol{p}\left(\boldsymbol{p}^{\prime}\right)$, given by

$$
p^{\prime i}(\boldsymbol{p})=\Lambda_{0}^{i}\left(m^{2}+\boldsymbol{p}^{2}\right)^{1 / 2}+\Lambda_{j}^{i} p^{j}
$$

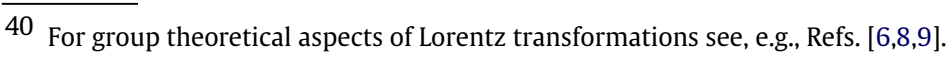




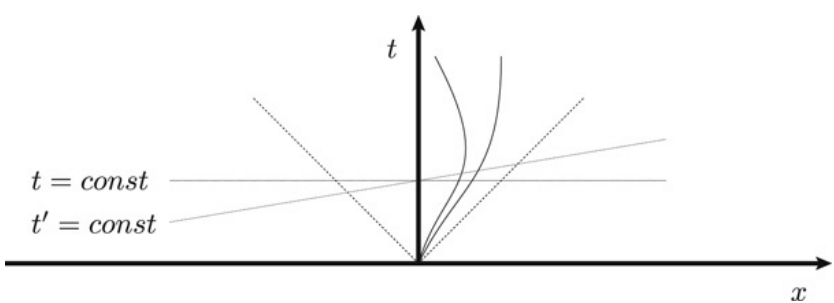

Fig. 3. The solid curves represent two world-lines starting at the same spacetime point (dashed lines indicate the forward lightcone) The one-particle phase space PDF $f(t, \boldsymbol{x}, \boldsymbol{p})$ measures the number of world-lines that pierce through the hyperplane " $t=$ constant" within the interval $[\boldsymbol{x}, \boldsymbol{x}+\mathrm{d} \boldsymbol{x}]$ while having momentum values in the range $[\boldsymbol{p}, \boldsymbol{p}+\mathrm{d} \boldsymbol{p}]$. Similarly, the PDF $f^{\prime}\left(t^{\prime}, \boldsymbol{x}^{\prime}, \boldsymbol{p}^{\prime}\right)$ measures the number of world-lines piercing through the hyperplane " $t^{\prime}=$ constant" within the interval $\left[\boldsymbol{x}^{\prime}, \boldsymbol{x}^{\prime}+\mathrm{d} \boldsymbol{x}^{\prime}\right]$ while having momenta in the range $\left[\boldsymbol{p}^{\prime}, \boldsymbol{p}^{\prime}+\mathrm{d} \boldsymbol{p}^{\prime}\right]$.

\subsubsection{Probability densities in special relativity}

With regard to the subsequent discussion, it is worthwhile to address a few subtleties concerning the definition and transformation behavior of probability density functions (PDFs) in special relativity [151,230].

Relativistic one-particle phase space distributions. To start with, we consider the one-particle phase space PDF $f(t, \boldsymbol{x}, \boldsymbol{p}) \geq 0$, where $(t, \boldsymbol{x}, \boldsymbol{p})$ are measured with respect to the inertial 'lab' frame $\Sigma$. For a relativistic many-particle system with conserved particle number $N$ (e.g., a gas of identical particles), the function $f$ can be defined operationally as follows [151]:

An observer $\mathcal{O}$, who is at rest in $\Sigma$ and observes the system at $\Sigma$-time $t$, finds

$$
N f(t, \boldsymbol{x}, \boldsymbol{p}) \mathrm{d}^{d} x \mathrm{~d}^{d} p
$$

particles in the $2 d$-dimensional phase space interval $[\boldsymbol{x}, \boldsymbol{x}+\mathrm{d} \boldsymbol{x}] \times[\boldsymbol{p}, \boldsymbol{p}+\mathrm{d} \boldsymbol{p}]$. Assuming that the dynamics of each particle is described by functions $\boldsymbol{X}_{r}(t)$ and $\boldsymbol{P}_{r}(t)$ in $\Sigma$, the fine-grained one-particle PDF $f$ of the $N$-particle system is defined by ${ }^{41}$

$$
f(t, \boldsymbol{x}, \boldsymbol{p})=N^{-1} \sum_{r=1}^{N} \delta\left(\boldsymbol{x}-\boldsymbol{X}_{r}(t)\right) \delta\left(\boldsymbol{p}-\boldsymbol{P}_{r}(t)\right) .
$$

From this definition it is evident that $f$ satisfies the $t$-simultaneous normalization condition

$$
1=\int \mathrm{d}^{d} x \mathrm{~d}^{d} p f(t, \boldsymbol{x}, \boldsymbol{p})
$$

Note that this integral is taken along the hyperplane " $t=$ constant" in $\Sigma$.

Alternatively, when considering, for example, the random motion of a single Brownian particle in a fluctuating medium, the quantity $f(t, \boldsymbol{x}, \boldsymbol{p}) \mathrm{d}^{d} x \mathrm{~d}^{d} p$ can be interpreted as the probability offinding the Brownian particle at lab time $t$ in $[\boldsymbol{x}, \boldsymbol{x}+\mathrm{d} \boldsymbol{x}] \times$ $[\boldsymbol{p}, \boldsymbol{p}+\mathrm{d} \boldsymbol{p}]$. In the latter case, it is usually assumed that a potential trajectory is realized with a certain $a$ priori probability. Mathematically, this idea is implemented by introducing latent variables $\omega$ in order to label the potential trajectories ${ }^{42}$ by writing $\boldsymbol{X}(t ; \omega)$ and $\boldsymbol{P}(t ; \omega)$. The assignment of a priori probabilities is equivalent to specifying a PDF $\Phi(\omega)$ on the set of the latent variables $\{\omega\}$. In this case, the phase space density $f$ in $\Sigma$ is defined by $[151,230]$

$$
f(t, \boldsymbol{x}, \boldsymbol{p})=\int \mathrm{d} \omega \Phi(\omega) \delta(\boldsymbol{x}-\boldsymbol{X}(t ; \omega)) \delta(\boldsymbol{p}-\boldsymbol{P}(t ; \omega)),
$$

and this $f$ is again subject to the normalization condition (88b).

Eq. (88) refer explicitly to the inertial rest frame $\Sigma$ of the observer $\mathcal{O}$. Now consider a second observer $\mathcal{O}^{\prime}$ at rest in another inertial frame $\Sigma^{\prime}$ that moves with constant velocity $\boldsymbol{w} \neq 0$ relative to $\Sigma$. Employing an analogous operational definition as $\mathcal{O}$, the moving observer $\mathcal{O}^{\prime}$ will measure another distribution $f^{\prime}\left(t^{\prime}, \boldsymbol{x}^{\prime}, \boldsymbol{p}^{\prime}\right)$ and so arises the question how the two functions $f^{\prime}\left(t^{\prime}, \boldsymbol{x}^{\prime}, \boldsymbol{p}^{\prime}\right)$ and $f(t, \boldsymbol{x}, \boldsymbol{p})$ are related to each other. In the nonrelativistic theory, the change from one inertial system to another does not affect the time coordinate; hence, one can use the standard transformation laws for PDFs in that case [see, e.g., Eq. (31)]. By contrast, the situation becomes more complicated in the relativistic theory, because now the definition of $f$ and $f^{\prime}$ is based on an observer-dependent notion of simultaneity: The measurements of $\mathcal{O}$ and $\mathcal{O}^{\prime}$ refer to the two different hyperplanes " $t=$ constant" and " $t$ ' $=$ constant" in Minkowski space, respectively. This is illustrated in Fig. 3.

\footnotetext{
41 Since the kinetic momentum $\boldsymbol{P}_{r}$ is uniquely linked to the velocity $\boldsymbol{V}_{r}(t):=\mathrm{d} \boldsymbol{X}_{r}(t) / \mathrm{d} t$, the definition (88a) formalizes the idea of classifying the particle curves $\boldsymbol{X}_{r}(t)$ according to their positions and time derivatives at time $t$.

42 For example, if the particle dynamics in $\Sigma$ is described by differential equations of the form $\mathrm{d} \boldsymbol{X}(t) / \mathrm{d} t=\boldsymbol{V}[\boldsymbol{X}(t), \boldsymbol{P}(t)], \mathrm{d} \boldsymbol{P}(t) / \mathrm{d} t=\boldsymbol{K}[\boldsymbol{X}(t), \boldsymbol{P}(t)]$ with given (well-behaved) functions $\boldsymbol{V}: \mathbb{R}^{d} \times \mathbb{R}^{d} \rightarrow \mathbb{R}^{d}$ and $\boldsymbol{K}: \mathbb{R}^{d} \times \mathbb{R}^{d} \rightarrow \mathbb{R}^{d}$, then a trajectory is uniquely determined by specifying the values $\boldsymbol{X}\left(t_{0}\right)=\boldsymbol{x}_{0}$ and $\boldsymbol{P}\left(t_{0}\right)=\boldsymbol{p}_{0}$ at some instant $t_{0}$ in $\Sigma$. In this case, one could choose $\omega=\left(\boldsymbol{x}_{0}, \boldsymbol{p}_{0}\right)$. More generally, $\omega$ could also label different realizations of some background field which affects the particle dynamics.
} 
In an insightful paper [151] published in 1969, van Kampen proved that the one-particle phase space PDF $f$ transforms as a Lorentz scalar, i.e., ${ }^{43}$

$$
f(t, \boldsymbol{x}, \boldsymbol{p})=f^{\prime}\left(t^{\prime}, \boldsymbol{x}^{\prime}, \boldsymbol{p}^{\prime}\right),
$$

where $(t, \boldsymbol{x}, \boldsymbol{p})$ and $\left(t^{\prime}, \boldsymbol{x}^{\prime}, \boldsymbol{p}^{\prime}\right)$ are connected by Lorentz transformations. His (first) proof of Eq. (89) merely uses an assumption about the uniqueness of particle trajectories and a reparameterization of the particles trajectories in terms of their invariant proper times (cf. Section 3 in [151]). As a consequence, Eq. (89) represents a generic kinematical result and applies to a broad class of interaction models. Furthermore, van Kampen showed that ${ }^{44}$

$$
\int \mathrm{d}^{d} x^{\prime} \mathrm{d}^{d} p^{\prime} f\left(t^{\prime}, \boldsymbol{x}^{\prime}, \boldsymbol{p}^{\prime}\right)=\int \mathrm{d}^{d} x \mathrm{~d}^{d} p f(t, \boldsymbol{x}, \boldsymbol{p}),
$$

implying that the function $f^{\prime}$ satisfies the $t^{\prime}$-simultaneous normalization condition

$$
1=\int \mathrm{d}^{d} x^{\prime} \mathrm{d}^{d} p^{\prime} f\left(t^{\prime}, \boldsymbol{x}^{\prime}, \boldsymbol{p}^{\prime}\right) .
$$

We next summarize a few consequences of Eq. (89).

Density-current vector. Equation (89) implies that the quantities

$$
\begin{aligned}
& \varrho(t, \boldsymbol{x})=\int \mathrm{d}^{d} p f(t, \boldsymbol{x}, \boldsymbol{p}) \\
& \boldsymbol{j}(t, \boldsymbol{x})=\int \mathrm{d}^{d} p f(t, \boldsymbol{x}, \boldsymbol{p}) \boldsymbol{v},
\end{aligned}
$$

where $\boldsymbol{v}=\boldsymbol{p} / \epsilon=\boldsymbol{p} / p^{0}$ is the particle velocity, can be combined into a density-current four-vector field $\bar{j}(\bar{x})=\left(j^{\alpha}\right)=(\varrho, \boldsymbol{j})$. As any covariant vector field, $\bar{j}$ transforms under a Lorentz transformation $\bar{x} \mapsto \bar{\chi}^{\prime}=\Lambda \bar{x}$ as

$$
\bar{j}^{\prime}\left(\bar{x}^{\prime}\right)=\Lambda \bar{j}\left(\Lambda^{-1} \bar{x}^{\prime}\right)=\Lambda \bar{j}(\bar{x}) .
$$

The four-vector character of $\left(j^{\alpha}\right)$ becomes particularly evident by rewriting Eq. (91) in the form

$$
j^{\alpha}(t, \boldsymbol{x})=\int \frac{\mathrm{d}^{d} p}{p^{0}} f(t, \boldsymbol{x}, \boldsymbol{p}) p^{\alpha},
$$

where

$$
\frac{\mathrm{d}^{d} p}{p^{0}}=\frac{\mathrm{d}^{d} p^{\prime}}{p^{\prime 0}}
$$

is invariant under Lorentz transformations [151,312]. Furthermore, as shown in Section 4 of van Kampen's paper [151], $j^{\alpha}$ satisfies the continuity equation

$$
\partial_{\alpha} j^{\alpha}=\frac{\partial}{\partial t} \varrho+\nabla \cdot \boldsymbol{j}=0
$$

stating the conservation of particle number or probability, in agreement with Eq. (90a). The kinematical proof of Eq. (95) as given in [151] does not require knowledge about the equations of motions, but uses only the existence of the particle trajectories.

Energy-momentum density tensor. Equation (93) can be generalized to define a symmetric energy-momentum (density) tensor field by $[151,312]$

$$
\theta^{\alpha \beta}(t, \boldsymbol{x})=\int \frac{\mathrm{d}^{d} p}{p^{0}} f(t, \boldsymbol{x}, \boldsymbol{p}) p^{\alpha} p^{\beta} .
$$

In particular, the "00"-component gives the (kinetic) energy density per particle. In contrast to the particle number conservation law (95), the conservation of the energy-momentum tensor is a dynamical property which requires knowledge about the equations of motions (see example in the next section).

In principle, "higher moment tensors" can be defined in a similar manner:

$$
\hat{\theta}^{\alpha \beta \gamma \cdots}(t, \boldsymbol{x})=\int \frac{\mathrm{d}^{d} p}{p^{0}} f(t, \boldsymbol{x}, \boldsymbol{p}) p^{\alpha} p^{\beta} p^{\gamma} \ldots
$$

However, conventional thermodynamic and hydrodynamic analysis $[164,177,477]$ usually focuses on relations involving $j^{\alpha}$ and $\theta^{\alpha \beta}$.

\footnotetext{
43 One can find several insufficient 'proofs' of Eq. (89) in the literature, cf. the discussion in [151,230]. In this context it is sometimes claimed, erroneously, that the phase space element $\mathrm{d}^{d} x \mathrm{~d}^{d} p$ is a Lorentz scalar; in Section 2 of their paper, Debbasch et al. [230] demonstrate that this is not true in general.

44 The result (90a) is nontrivial due the fact that the integrals refer to different hyperplanes " $t=$ constant" and " $t$ ' $=$ constant" in Minkowski spacetime, respectively. The proof of Eq. (90a) follows from Gauss' theorem, by making use of the fact that the absolute particle velocities are bounded by the speed of light $c=1$, cf. Eqs. (34) and (35) in [151].
} 
Marginal momentum distributions. The zero-component $\varrho(t, \boldsymbol{x})$ of the density-current vector $\bar{j}(\bar{x})$, defined in Eq. (91a), can be interpreted as the marginal distribution of the particle positions. Similarly, one may define marginal momentum distributions with respect to $\Sigma$ and $\Sigma^{\prime}$ by

$$
\begin{aligned}
& \phi(t, \boldsymbol{p})=\int \mathrm{d}^{d} x f(t, \boldsymbol{x}, \boldsymbol{p}), \\
& \phi^{\prime}\left(t^{\prime}, \boldsymbol{p}^{\prime}\right)=\int \mathrm{d}^{d} x^{\prime} f^{\prime}\left(t^{\prime}, \boldsymbol{x}^{\prime}, \boldsymbol{p}^{\prime}\right) .
\end{aligned}
$$

Again, $\phi$ and $\phi^{\prime}$ refer to different hyperplanes in Minkowski spacetime, respectively. Thus, in general, it is impossible to calculate $\phi^{\prime}$ from $\phi$ alone or vice versa. There exist, however, a few exceptions such as, for example, a system of freely moving, identical particles (i.e., no interactions, no external fields, no walls). In any inertial frame, such a collection of particles is described by a time-dependent ${ }^{45}$ one-particle phase space PDF $f$ satisfying [151]

$$
f(t, \boldsymbol{x}, \boldsymbol{p})=f(0, \boldsymbol{x}-\boldsymbol{v} t, \boldsymbol{p}),
$$

where $\boldsymbol{v}=\boldsymbol{p} /\left(m^{2}+\boldsymbol{p}^{2}\right)^{1 / 2}$ is the velocity. Moreover, since the individual particle momenta do not change, the marginal momentum distribution must be time-independent in any inertial frame. In particular, in this case - and only in this case it is true that each particle observed by $\mathcal{O}$ as having momentum $\boldsymbol{p}$ is observed by $\mathcal{O}^{\prime}$ as having momentum $\boldsymbol{p}^{\prime}$, i.e., [151]

$$
\phi(\boldsymbol{p}) \mathrm{d}^{d} p=\phi^{\prime}\left(\boldsymbol{p}^{\prime}\right) \mathrm{d}^{d} p^{\prime},
$$

where $\boldsymbol{p}^{\prime}(\boldsymbol{p})$ is the restricted Lorentz transformation from Eq. (87). Taking into account that $\mathrm{d}^{d} p^{\prime}=\left(p^{\prime 0} / p^{0}\right) \mathrm{d}^{d} p$, cf. Eq. (94), one thus obtains

$$
\left(m^{2}+\boldsymbol{p}^{2}\right)^{1 / 2} \phi(\boldsymbol{p})=\left(m^{2}+\boldsymbol{p}^{\prime 2}\right)^{1 / 2} \phi^{\prime}\left(\boldsymbol{p}^{\prime}\right) .
$$

It should be stressed again that this formula holds true only in the case of an unconfined, non-interacting systems; it is not valid anymore in the presence of a confinement (see example in Section 3.2).

Multi-particle distributions. The discussion in the remainder mostly concerns one-particle distributions. Nonetheless, we mention that Eq. (89) can be generalized to the case of $N$-particle phase space PDFs $f_{N}$, yielding [151]

$$
f_{N}\left(t_{1}, \boldsymbol{x}_{1}, \boldsymbol{p}_{1} ; \ldots ; t_{N}, \boldsymbol{x}_{N}, \boldsymbol{p}_{N}\right)=f_{N}^{\prime}\left(t_{1}^{\prime}, \boldsymbol{x}_{1}^{\prime}, \boldsymbol{p}_{1}^{\prime} ; \ldots ; t_{N}^{\prime}, \boldsymbol{x}_{N}^{\prime}, \boldsymbol{p}_{N}^{\prime}\right),
$$

where for $n=1, \ldots, N$ the coordinates $\left(t_{n}^{\prime}, \boldsymbol{x}_{n}^{\prime}, \boldsymbol{p}_{n}^{\prime}\right)$ and $\left(t_{n}, \boldsymbol{x}_{n}, \boldsymbol{p}_{n}\right)$ are connected by a Lorentz-transformation, and $f_{N}$ is the multiple-time probability density for the lab observer $\mathcal{O}$ to observe particle 1 at time $t_{1}$ near $\left(\boldsymbol{x}_{1}, \boldsymbol{p}_{1}\right)$, and particle 2 at time $t_{2}$ near $\left(\boldsymbol{x}_{2}, \boldsymbol{p}_{2}\right)$, etc.

The above results clarify the transformation behavior of PDFs in special relativity, but they do not yet answer the question as to which PDF provides the correct description for a given physical system as, for example, a relativistic gas in equilibrium. The latter problem will be addressed in the next part.

\subsection{Stationary systems with confinement}

Of particular relevance in equilibrium thermodynamics are confined systems that can be described by an isotropic, stationary PDF $f(t, \boldsymbol{x}, \boldsymbol{p})=\varphi(\boldsymbol{x}, \boldsymbol{p})$ in a specific inertial frame $\Sigma$. A typical example is an equilibrated gas, enclosed in a container box which is at rest in the lab frame $\Sigma$. In standard Brownian motion theory, such systems often play the role of the heat bath.

\subsubsection{General remarks}

Lab frame. In the lab frame $\Sigma$, a spatially homogeneous gas is described by a PDF of the form

$$
\varphi(\boldsymbol{x}, \boldsymbol{p})=V^{-1} \ell(\boldsymbol{x} ; \mathbb{V}) \phi(\boldsymbol{p}),
$$

where $V=\lambda_{d}(\mathbb{V})$ is the rest volume number (i.e., the Lebesgue measure [478]) of the spatial container region $\mathbb{V} \subset \mathbb{R}^{d}$ in $\Sigma$, and $\ell(\boldsymbol{x} ; \mathbb{V})$ denotes the indicator function of the box, i.e.,

$$
\ell(\boldsymbol{x} ; \mathbb{V}):= \begin{cases}1, & \boldsymbol{x} \in \mathbb{V} \\ 0, & \boldsymbol{x} \notin \mathbb{V}\end{cases}
$$

\footnotetext{
$\overline{45}$ We assume that at least two particles have different velocities.
} 
To be more specific, we consider a cubic container of length $L$ such that $\mathbb{V}=[-L / 2, L / 2]^{d}$. In this case, $V=\lambda_{d}(\mathbb{V})=L^{d}$ and the indicator function can be expressed as

$$
\ell(\boldsymbol{x} ; \mathbb{V}):=\prod_{i=1}^{d} \Theta\left(L / 2+x^{i}\right) \Theta\left(L / 2-x^{i}\right) .
$$

Isotropy and stationarity of the gas in the lab frame $\Sigma$ require the marginal momentum distribution to be rotationally invariant, $\phi(\boldsymbol{p})=\hat{\phi}(|\boldsymbol{p}|)$, yielding for the four-current $\left(j^{\alpha}\right)=(\varrho, \boldsymbol{j})$ in $\Sigma$

$$
\begin{aligned}
& \varrho(t, \boldsymbol{x})=V^{-1} \ell(\boldsymbol{x} ; \mathbb{V}), \\
& \boldsymbol{j}(t, \boldsymbol{x})=\mathbf{0} .
\end{aligned}
$$

It is obvious that this current satisfies the continuity equation (95), $\partial_{\alpha} j^{\alpha}=0$. Furthermore, due to the isotropy of the momentum distribution $\phi$ in $\Sigma$, the energy-momentum tensor (96) becomes diagonal with components given by ${ }^{46}$

$$
\theta^{\alpha \beta}(t, \boldsymbol{x})=V^{-1} \ell(\boldsymbol{x} ; \mathbb{V}) \begin{cases}\langle\epsilon\rangle, & \alpha=\beta=0, \\ \langle\boldsymbol{p} \cdot \boldsymbol{v}\rangle / d, & \alpha=\beta=1, \ldots, d, \\ 0, & \alpha \neq \beta .\end{cases}
$$

Here, we have defined the one-particle mean values

$$
\begin{aligned}
& \langle\epsilon\rangle:=\int \mathrm{d}^{d} p \phi(\boldsymbol{p}) \epsilon, \\
& \langle\boldsymbol{p} \cdot \boldsymbol{v}\rangle:=\int \mathrm{d}^{d} p \phi(\boldsymbol{p}) \boldsymbol{p} \cdot \boldsymbol{v}
\end{aligned}
$$

with $\epsilon(\boldsymbol{p})=\left(\boldsymbol{p}^{2}+m^{2}\right)^{1 / 2}$ denoting the energy of a gas particle, and $\boldsymbol{v}(\boldsymbol{p})=\boldsymbol{p} / \epsilon$ the velocity.

It is worthwhile to calculate the four-divergence of the energy momentum tensor:

$$
\partial_{\alpha} \theta^{\alpha \beta}=(V d)^{-1}\langle\boldsymbol{p} \cdot \boldsymbol{v}\rangle \begin{cases}0, & \beta=0, \\ \partial_{i} \ell(\boldsymbol{x} ; \mathbb{V}), & \beta=i,\end{cases}
$$

where, in the case of a cubic box $V:=\lambda_{d}(\mathbb{V})=[-L / 2, L / 2]^{d}$, we find

$$
\partial_{i} \ell(\boldsymbol{x} ; \mathbb{V})=\left[\delta\left(L / 2+x^{i}\right)-\delta\left(L / 2-x^{i}\right)\right] \prod_{j \neq i} \Theta\left(L / 2+x^{j}\right) \Theta\left(L / 2-x^{j}\right) ;
$$

i.e., the boundaries are sources of stress [152]. ${ }^{47}$

To illustrate the meaning of the energy-momentum tensor $\theta^{\alpha \beta}$, consider the mean (integrated) energy-momentum vector

$$
\left\langle p^{\beta}\right\rangle_{t}:=\int \mathrm{d}^{d} x \mathrm{~d}^{d} p f(t, \boldsymbol{x}, \boldsymbol{p}) p^{\beta} .
$$

This quantity can be rewritten in terms of $\theta^{\alpha \beta}$ as follows:

$$
\left\langle p^{\beta}\right\rangle_{t}=\int \mathrm{d}^{d} x \theta^{0 \beta}(t, \boldsymbol{x})=\int_{t} \mathrm{~d} \sigma_{\alpha} \theta^{\alpha \beta}(t, \boldsymbol{x}) .
$$

The directed surface element normal to the hyperplane " $t=$ constant" in $\Sigma$-coordinates is given by $\left(\mathrm{d} \sigma_{\alpha}\right)=\left(\mathrm{d}^{d} x, \mathbf{0}\right)$; cf. Appendix B. Thus, a $d$-dimensional spatial integration in $\Sigma$ is equivalent to a surface integral over the hyperplane " $t=$ constant" in $(1+d)$-dimensional Minkowski space. For the energy-momentum tensor (106) one finds explicitly

$$
\left\langle p^{\beta}\right\rangle_{t}= \begin{cases}\langle\epsilon\rangle, & \beta=0 \\ 0, & \beta \neq 0 .\end{cases}
$$

It is important to note that $\left\langle p^{\beta}\right\rangle_{t}$ is a non-local quantity, as it represents a sum over components of the energy-momentum tensor at different spacetime points. As emphasized by Gamba [476] and discussed below, this aspect becomes relevant if one considers the question of how a moving observer $\mathcal{O}^{\prime}$ could, in principle, determine $\left\langle p^{\beta}\right\rangle_{t}$.

\footnotetext{
46 As usual, we denote by $\boldsymbol{p} \cdot \boldsymbol{v}$ the ordinary Euclidean scalar product of two $d$-dimensional vectors $\boldsymbol{p}=\left(p^{i}\right)$ and $\boldsymbol{v}=\left(v^{i}\right)$, i.e., $\boldsymbol{p} \cdot \boldsymbol{v}:=\sum_{i=1}^{d} p^{i} v^{i}$. Moreover, we abbreviate $\boldsymbol{p}^{2}:=\boldsymbol{p} \cdot \boldsymbol{p}=p_{i} p^{i}=\sum_{i=1}^{d} p^{i} p^{i}$.

47 Usually, it is assumed that the momentum conservation violation of the gas, $\partial_{\alpha} \theta_{\text {gas }}^{\alpha \beta} \neq 0$, is compensated for by the energy-momentum tensor $\theta_{\text {conf }}^{\alpha \beta}$ of the confinement (environment), i.e., $\partial_{\alpha}\left(\theta_{\text {gas }}^{\alpha \beta}+\theta_{\text {conf }}^{\alpha \beta}\right) \equiv 0$; a similar problem occurs in continuum models of the electron, cf. [479].
} 
Moving frame. Consider an observer $\mathcal{O}^{\prime}$ who is at rest in a frame $\Sigma^{\prime}$ that moves at velocity $\boldsymbol{w}=(w, 0, \ldots, 0)$ along the $x^{1}$-axis of $\Sigma$. Denoting the spacetime coordinates and four-momentum in $\Sigma$ by $\left(x^{\alpha}\right)=\left(t, x^{1}, \ldots, x^{d}\right)$ and $\left(p^{\alpha}\right)=$ $\left(\epsilon, p^{1}, \ldots, p^{d}\right)=(\epsilon, \boldsymbol{p})$ and those in $\Sigma^{\prime}$ by $\left(x^{\prime \alpha}\right)=\left(t^{\prime}, x^{\prime 1}, \ldots, x^{\prime d}\right)$ and $\left(p^{\prime \alpha}\right)=\left(\epsilon^{\prime}, p^{\prime 1}, \ldots, p^{\prime d}\right)=\left(\epsilon^{\prime}, \boldsymbol{p}^{\prime}\right)$, we obtain from Eqs. (80a), (83) and (87) the explicit Lorentz transformations

$$
\begin{aligned}
t^{\prime} & =\gamma\left(t-w x^{1}\right) & t & =\gamma\left(t^{\prime}+w x^{\prime 1}\right) \\
x^{\prime 1} & =\gamma\left(-w t+x^{1}\right) & x^{1} & =\gamma\left(w t^{\prime}+x^{\prime 1}\right) \\
\epsilon^{\prime} & =\gamma\left(\epsilon-w p^{1}\right) & \epsilon & =\gamma\left(\epsilon^{\prime}+w p^{\prime 1}\right) \\
p^{\prime 1} & =\gamma\left(-w \epsilon+p^{1}\right) & p^{1} & =\gamma\left(w \epsilon^{\prime}+p^{\prime 1}\right) \\
x^{\prime j} & =x^{j} & p^{j} & =p^{\prime j} \quad j=2, \ldots, d,
\end{aligned}
$$

where $\gamma=\gamma(w)=\left(1-w^{2}\right)^{-1 / 2}$ is the Lorentz factor; $\epsilon=\left(m^{2}+\boldsymbol{p}^{2}\right)^{1 / 2}$ and $\epsilon^{\prime}=\left(m^{2}+\boldsymbol{p}^{\prime 2}\right)^{1 / 2}$ denotes the relativistic energy, respectively. From Eq. (109) one can obtain an explicit expression for the phase space PDF $f^{\prime}$ measured by the moving observer. If the observer moves at three-velocity $\boldsymbol{w}=(w, 0, \ldots, 0)$ through the lab frame, then from her point of view, the container box moves at velocity

$$
\boldsymbol{w}^{\prime}=\left(w^{\prime}, 0, \ldots, 0\right)=(-w, 0, \ldots, 0)=-\boldsymbol{w}
$$

through $\Sigma^{\prime}$. By inserting Eqs. (109) and (110) into Eq. (89) for a stationary lab distribution of the form (103a), one obtains the $\operatorname{PDF} f^{\prime}$ in the moving frame $\Sigma^{\prime}$ as

$$
f^{\prime}\left(t^{\prime}, \boldsymbol{x}^{\prime}, \boldsymbol{p}^{\prime}\right)=V^{-1} \ell\left(\gamma\left(-w^{\prime} t^{\prime}+x^{\prime 1}\right), x^{\prime 2} \ldots, x^{\prime d} ; \mathbb{V}\right) \phi\left(\gamma\left(-w^{\prime} \epsilon^{\prime}+p^{\prime 1}\right), p^{\prime 2}, \ldots, p^{\prime d}\right) .
$$

While the phase space PDF $f$ was stationary in $\Sigma$, the associated PDF $f^{\prime}$ is not stationary in $\Sigma^{\prime}$ due to the motion of the container box. ${ }^{48}$ Considering the representation (104) of the indicator function $\ell$ in $\Sigma$, one can introduce a corresponding quantity $\ell^{\prime}$ in $\Sigma^{\prime}$ by

$$
\begin{aligned}
\ell^{\prime}\left(t^{\prime}, \boldsymbol{x}^{\prime} ; \mathbb{V}\right) & :=\ell\left(\gamma\left(-w^{\prime} t^{\prime}+x^{\prime 1}\right), x^{\prime 2}, \ldots, x^{\prime d} ; \mathbb{V}\right) \\
& =\Theta\left(L / 2+\gamma\left(x^{\prime 1}-w^{\prime} t^{\prime}\right)\right) \Theta\left(L / 2-\gamma\left(x^{\prime 1}-w^{\prime} t^{\prime}\right)\right) \prod_{i=2}^{d} \Theta\left(L / 2+x^{\prime i}\right) \Theta\left(L / 2-x^{\prime i}\right) \\
& =\Theta\left(L^{\prime} / 2+\left(x^{\prime 1}-w^{\prime} t^{\prime}\right)\right) \Theta\left(L^{\prime} / 2-\left(x^{\prime 1}-w^{\prime} t^{\prime}\right)\right) \prod_{i=2}^{d} \Theta\left(L / 2+x^{\prime i}\right) \Theta\left(L / 2-x^{\prime i}\right),
\end{aligned}
$$

where $L^{\prime}=L / \gamma$ is the Lorentz-contracted length in $x^{\prime 1}$-direction. By means of Eq. (112), one can express the components of the conserved four-current $\left(j^{\prime \alpha}\right)=\left(\varrho^{\prime}, \boldsymbol{j}^{\prime}\right)=(\gamma \varrho,-\gamma \boldsymbol{w} \varrho)$ in $\Sigma^{\prime}$ as [cf. Eq. (105)]

$$
\begin{aligned}
& \varrho^{\prime}\left(t^{\prime}, \boldsymbol{x}^{\prime}\right)=V^{\prime-1} \ell^{\prime}\left(t^{\prime}, \boldsymbol{x}^{\prime} ; \mathbb{V}\right) \\
& \boldsymbol{j}^{\prime}\left(t^{\prime}, \boldsymbol{x}^{\prime}\right)=V^{\prime-1} \ell^{\prime}\left(t^{\prime}, \boldsymbol{x}^{\prime} ; \mathbb{V}\right) \boldsymbol{w}^{\prime}=\varrho^{\prime} \boldsymbol{w}^{\prime},
\end{aligned}
$$

with $V^{\prime}:=V / \gamma$ denoting the Lorentz-contracted box volume in the moving frame $\Sigma^{\prime}$. By inserting the expression (112) into Eq. (111), one may perform a volume integration along the hyperplane " $t$ ' $=$ constant" to obtain the marginal momentum density in the moving frame as [151]

$$
\phi^{\prime}\left(\boldsymbol{p}^{\prime}\right):=\int \mathrm{d}^{d} x^{\prime} f^{\prime}\left(t^{\prime}, \boldsymbol{x}^{\prime}, \boldsymbol{p}^{\prime}\right)=\gamma^{-1} \phi\left(\boldsymbol{p}\left(\boldsymbol{p}^{\prime}\right)\right) .
$$

This result was already obtained by Dirac [480] in 1924; it is sometimes written in the equivalent form [151] ${ }^{49}$

$$
\phi^{\prime}\left(\boldsymbol{p}^{\prime}\right) / V^{\prime}=\phi(\boldsymbol{p}) / V \text {. }
$$

As anticipated above, Eq. (114) differ from the corresponding result (101) for an unconfined system of non-interacting particles. At first sight, it may seem surprising that the presence of the box alters the transformation properties of the momentum distribution. The underlying physical explanation is that the observations by $\mathcal{O}$ and $\mathcal{O}^{\prime}$ are not synchronous and that in the time between their observations some particles collide with the container walls. ${ }^{50}$

\footnotetext{
48 In fact, this also happens in the nonrelativistic case.

49 Experimentally, the momentum PDF $\phi(\boldsymbol{p})$ can be determined by collecting the momentum values $\boldsymbol{p}_{r}(t)$ of the $N$ gas particles, measured at the same instant of time $t$ in $\Sigma$, into a histogram. Similarly, the function $\phi^{\prime}\left(\boldsymbol{p}^{\prime}\right)$ can be determined from a histogram of the momentum values $\boldsymbol{p}_{r}^{\prime}\left(t^{\prime}\right)$, measured at the same time $t^{\prime}$ in $\Sigma^{\prime}$.

50 Cf. discussion in Section 6 of van Kampen's paper [151].
} 
The marginal momentum distributions present another example of non-locally defined quantities. In the history of relativity, such quantities have been a source of considerable confusion. ${ }^{51}$ Pitfalls may be avoided by keeping in mind that [476]:

In general, non-locally defined quantities are not connected by Lorentz transformations if they refer to different subsets (e.g., hyperplanes) in Minkowski spacetime. Therefore, a complete measuring instruction for a nonlocal observable must include a statement about the set of events over which the quantity is defined.

To illustrate this, we may consider the non-locally defined vector

$$
\left\langle p^{\prime \beta}\right\rangle_{t^{\prime}}:=\int \mathrm{d}^{d} x^{\prime} \mathrm{d}^{d} p^{\prime} f^{\prime}\left(t^{\prime}, \boldsymbol{x}^{\prime}, \boldsymbol{p}^{\prime}\right) p^{\beta}=\int_{t^{\prime}} \mathrm{d} \sigma_{\alpha}^{\prime} \theta^{\prime \alpha \beta}\left(t^{\prime}, \boldsymbol{x}^{\prime}\right),
$$

representing a surface integral along the hyperplane " $t$ ' $=$ constant" ${ }^{52}$ Although the average four-momentum vector $\left\langle p^{\prime \beta}\right\rangle_{t^{\prime}}$ from Eq. (115a) looks quite similar to $\left\langle p^{\beta}\right\rangle_{t}$ from Eq. (108), the crucial difference is given by the fact that these two objects refer to two distinct hyperplanes, respectively, as signaled by the different labels $t$ and $t^{\prime}$. For the energy-momentum tensor (106), the rhs of Eq. (115a) can be evaluated to give [218]

$$
\left\langle p^{\prime \beta}\right\rangle_{t^{\prime}}= \begin{cases}\gamma\left(\langle\epsilon\rangle+w^{2}\langle\boldsymbol{p} \cdot \boldsymbol{v}\rangle / d\right), & \beta=0 \\ -\gamma w(\langle\epsilon\rangle+\langle\boldsymbol{p} \cdot \boldsymbol{v}\rangle / d), & \beta=1 \\ 0, & \beta>1\end{cases}
$$

Upon comparing this result with Eq. (108c), we note that $\left\langle p^{\beta}\right\rangle_{t}$ and $\left\langle p^{\prime}\right\rangle_{t^{\prime}}$ are indeed not related by a Lorentz transformation, i.e.,

$$
\left\langle p^{\prime \alpha}\right\rangle_{t^{\prime}} \neq\left\langle p^{\prime \alpha}\right\rangle_{t}=\Lambda_{\beta}^{\alpha}\left\langle p^{\beta}\right\rangle_{t}
$$

This inequality is, in fact, caused by the non-vanishing divergence of the energy-momentum vector in the presence of a container, cf. Eq. (107). The actual Lorentz transformation $\left\langle p^{\prime}{ }^{\beta}\right\rangle_{t}$ of $\left\langle p^{\beta}\right\rangle_{t}$ is obtained by keeping the underlying hypersurface " $t=$ constant" fixed $[178,476]$, i.e.,

$$
\left\langle p^{\prime \beta}\right\rangle_{t}:=\Lambda_{\mu}^{\beta} \int \mathrm{d}^{d} x \theta^{0 \mu}(t, \boldsymbol{x})=\Lambda_{\mu}^{\beta} \int_{t} \mathrm{~d} \sigma_{\alpha} \theta^{\alpha \mu}=\int_{t} \mathrm{~d} \sigma_{\alpha}^{\prime} \theta^{\prime \alpha \beta},
$$

producing the expected result

$$
\left\langle p^{\prime \beta}\right\rangle_{t}= \begin{cases}\gamma\langle\epsilon\rangle, & \beta=0 \\ -\gamma w\langle\epsilon\rangle, & \beta=1 \\ 0, & \beta>1\end{cases}
$$

With regard to experimental observations this means that, in order to determine $\left\langle p^{\prime}{ }^{\beta}\right\rangle_{t}$, a moving observer $\mathcal{O}^{\prime}$ must first reconstruct the momentum values on the hyperplane " $t=$ constant" from her data before being able to compare with the averages $\left\langle p^{\beta}\right\rangle_{t}$ of a lab observer $\mathcal{O}$.

This gives rise to a bit of a dilemma when attempting to formulate a relativistic thermodynamic theory that is based on global system averages such as, for example, the total internal energy, momentum, etc.:

- Following Planck [121] and Einstein [122], one can build a theory based on observer-simultaneously defined quantities, as those in Eq. (115b), but this leads to a loss of covariance because different observers would use different hyperplanes depending on their velocities.

\footnotetext{
51 Gamba [476] gives a detailed list of examples, including the Lorentz contraction of length: "Only rarely is it pointed out clearly that the measurements of the two observers ... do not refer to the same set of events. The 'ends' of the rod being taken as contemporary for both observer at rest and moving observer, are in fact different points in the four-dimensional (absolute) spacetime. Once this is clearly indicated, the accepted definition [of length] is not particularly harmful".

52 As discussed in more detail in Appendix B, for a surface integral along the hyperplane " $t$ ' $=$ constant" in $\Sigma^{\prime}$ one finds in $\Sigma^{\prime}$-coordinates
}

$$
\int_{t^{\prime}} \mathrm{d} \sigma_{\alpha}^{\prime} \theta^{\prime \alpha \beta \cdots}\left(t^{\prime}, \boldsymbol{x}^{\prime}\right)=\int \mathrm{d}^{d} x^{\prime} \theta^{\prime 0 \beta \cdots}\left(t^{\prime}, \boldsymbol{x}^{\prime}\right) .
$$


- Alternatively, one can define thermodynamic quantities with respect to a specific, fixed hyperplane to obtain a manifestly covariant formalism [178]. In this case, however, the question arises as to which hyperplane is the most appropriate one (cf. discussion below).

These difficulties can, in principle, be avoided by formulating a thermodynamic field theory $[164,170,173,177,183$, $290,477,481]$ in terms of local tensorial quantities as, e.g., $j^{\alpha}$ and $\theta^{\alpha \beta}$. In view of the fact that traditional nonrelativistic thermodynamics [287,482] intends to describe a many-particle system by means of a few macroscopic control parameters, one could argue that such a field theoretic approach is already somewhat closer to hydrodynamics.

\subsubsection{Jüttner gas}

The above results apply to an arbitrary stationary momentum PDF $\phi(\boldsymbol{p})$ in the lab frame $\Sigma$. With regard to Brownian motion theory, one is particularly interested in thermal equilibrium distributions. When postulating relativistic Langevin equations $[11,17,18,20]$, these distributions must be known in advance in order to correctly specify the relativistic fluctuation-dissipation relation. Similarly, thermal equilibrium distributions are required as an input, if one wishes to derive Langevin-type equations from microscopic models, cf. Section 2.2. ${ }^{53}$

At the beginning of the last century, it was commonly accepted that a dilute (quasi-ideal) gas in equilibrium is described by the Maxwellian velocity PDF [187]

$$
\psi_{\mathrm{M}}(\boldsymbol{v} ; m, \beta, d)=[\beta m /(2 \pi)]^{d / 2} \exp \left(-\beta m \boldsymbol{v}^{2}\right),
$$

or, equivalently, by the one-particle momentum distribution

$$
\phi_{\mathrm{M}}(\boldsymbol{p} ; m, \beta, d)=[\beta /(2 \pi m)]^{d / 2} \exp \left[-\beta \boldsymbol{p}^{2} /(2 m)\right],
$$

where $m$ is the rest mass of a gas particle, $\boldsymbol{v}=\boldsymbol{p} / m \in \mathbb{R}^{d}$ the nonrelativistic velocity, and $\mathcal{T}=\left(k_{\mathrm{B}} \beta\right)^{-1}$ the temperature. After Einstein [2,3] had formulated his theory of special relativity in 1905, Planck and others noted immediately that the distribution (117) is in conflict with the fundamental relativistic postulate that velocities cannot exceed the light speed $c$. In 1911 a solution to this problem was put forward by Jüttner [186], who proposed to replace Maxwell's PDF by

$$
\psi_{\mathrm{J}}(\boldsymbol{v} ; m, \beta, d)=\mathcal{Z}_{d}^{-1} m^{d} \gamma(\boldsymbol{v})^{2+d} \exp [-\beta m \gamma(\boldsymbol{v})] \Theta(1-|\boldsymbol{v}|),
$$

yielding for the relativistic momentum $\boldsymbol{p}=m \boldsymbol{v} \gamma(\boldsymbol{v})$ the PDF

$$
\phi_{\mathrm{J}}(\boldsymbol{p} ; m, \beta)=\mathcal{Z}_{d}^{-1} \exp \left[-\beta\left(m^{2}+\boldsymbol{p}^{2}\right)^{1 / 2}\right] .
$$

Similar to the Maxwell distribution (117), Jüttner's distribution (118) refers to a lab frame $\Sigma$ where the vessel, enclosing the gas, is at rest. For space dimensions $d=1,2,3$, the normalization constant

$$
\mathcal{Z}_{d}(m, \beta)=\int \mathrm{d}^{d} p \exp \left[-\beta\left(m^{2}+\boldsymbol{p}^{2}\right)^{1 / 2}\right]
$$

can be expressed as [186]

$$
\begin{aligned}
& Z_{1}=2 m K_{1}(\beta m), \\
& Z_{2}=2 \pi m^{2} \exp (-\beta m)(1+\beta m) /(\beta m)^{2}, \\
& Z_{3}=4 \pi m^{3} K_{2}(\beta m) /(\beta m),
\end{aligned}
$$

with $K_{n}(z)$ denoting modified Bessel functions of the second kind [486]. The average energy per particle is obtained by logarithmic differentiation

$$
\langle\epsilon\rangle_{d}=-\frac{\partial}{\partial \beta} \ln \mathcal{Z}_{d},
$$

yielding

$$
\begin{aligned}
\langle\epsilon\rangle_{1} & =m \frac{K_{0}(\beta m)+K_{2}(\beta m)}{2 K_{1}(\beta m)}, \\
\langle\epsilon\rangle_{2} & =\frac{2}{\beta}+\frac{m^{2} \beta}{1+m \beta}, \\
\langle\epsilon\rangle_{3} & =\frac{3}{\beta}+m \frac{K_{1}(\beta m)}{K_{2}(\beta m)},
\end{aligned}
$$

\footnotetext{
53 Knowledge of the relativistic equilibrium distributions is essential for the correct interpretation of experimental observations in high energy and astrophysics [318,319,326,411]. Examples include thermalization processes in heavy ion collision experiments [318,319] and ultra-relativistic plasma beams [309,326], or the relativistic Sunyaev-Zel'dovich (SZ) effect [411], describing the distortion of the cosmic microwave background (CMB) radiation spectrum due to the interaction of CMB photons with hot electrons in clusters of galaxies [483-485]. The predicted strength of the spectral distortions and the cosmological parameters inferred from the SZ effect depend on the assumed velocity distribution of the relativistic electrons [411].
} 
and exhibiting the limiting behavior

$$
\begin{aligned}
& \lim _{\beta \rightarrow \infty}\langle\epsilon\rangle_{d}=m, \\
& \lim _{\beta \rightarrow 0} \beta\langle\epsilon\rangle_{d}=d .
\end{aligned}
$$

Moreover, one can show that, for arbitrary space dimensions $d$ the expectation value $\langle\boldsymbol{p} \cdot \boldsymbol{v}\rangle$ is independent of the mass $m$; more precisely

$$
\langle\boldsymbol{p} \cdot \boldsymbol{v}\rangle=\left\langle\boldsymbol{p}^{2} / \epsilon\right\rangle=d / \beta,
$$

which allows one to regard $\langle\boldsymbol{p} \cdot \boldsymbol{v}\rangle$ as a statistical thermometer (cf. discussion below).

Maximum (relative) entropy principle. Jüttner [186] originally derived the distribution (118) from a maximum entropy principle $[219,242,475]$ by postulating that, for a sufficiently large particle number $N \gg 1$, the one-particle equilibrium distribution in phase space $\varphi(\boldsymbol{x}, \boldsymbol{p})$ be a maximizer of the Boltzmann-Gibbs-Shannon (BGS) entropy ${ }^{54}$

$$
\mathfrak{S}\left[\phi \mid h^{-d}\right]=-k_{\mathrm{B}} \int_{\mathbb{V}} \mathrm{d}^{d} x \int \mathrm{d}^{d} p \varphi(\boldsymbol{x}, \boldsymbol{p}) \ln \left[h^{d} \varphi(\boldsymbol{x}, \boldsymbol{p})\right],
$$

while satisfying the constraints

$$
\begin{aligned}
& 1=\int_{\mathbb{V}} \mathrm{d}^{d} x \int \mathrm{d}^{d} p \varphi(\boldsymbol{x}, \boldsymbol{p}), \\
& \langle\epsilon\rangle=\int_{\mathbb{V}} \mathrm{d}^{d} x \int \mathrm{d}^{d} p \varphi(\boldsymbol{x}, \boldsymbol{p}) \epsilon,
\end{aligned}
$$

for a given mean energy value per particle $\langle\epsilon\rangle=\mathscr{E} / N$. Pursuing a similar approach, Jüttner later also derived the corresponding expressions for relativistic quantum gases obeying Bose and Fermi statistics [194]. ${ }^{55}$

Microcanonical approach. An alternative way [123,227,242] of justifying the Jüttner distribution (118) is based on the microcanonical ensemble. To illustrate this, consider a dilute system of weakly interacting relativistic particles enclosed in a vessel $\mathbb{V} \subset \mathbb{R}^{d}$ of volume $V=\lambda_{d}(\mathbb{V})$ which is at rest in the lab frame $\Sigma$. Assume that, with respect to $\Sigma$, this system can - in some approximation - be described by a truncated Hamiltonian of the form

$$
H\left(\boldsymbol{x}_{1}, \ldots, \boldsymbol{p}_{N}\right)=\sum_{s=1}^{N}\left(m^{2}+\boldsymbol{p}_{s}^{2}\right)^{1 / 2}=\mathcal{E},
$$

complemented by the condition of elastic reflections at the boundaries of the vessel. By writing (124a) one demands that the energy stored in the interaction is negligible compared with the energy carried by the particles, so that $\mathscr{E}$ is approximately conserved. The corresponding microcanonical $N$-particle distribution is given by the PDF

$$
f_{N}^{\mathrm{MC}}\left(\boldsymbol{x}_{1}, \ldots, \boldsymbol{p}_{N}\right)=\omega^{-1} \begin{cases}\delta\left(\mathcal{E}-H\left(\boldsymbol{x}_{1}, \ldots \boldsymbol{p}_{\mathrm{N}}\right)\right), & \boldsymbol{x}_{s} \in \mathbb{V}, \\ 0, & \boldsymbol{x}_{s} \notin \mathbb{V} .\end{cases}
$$

The normalization constant $\omega=\omega(\mathcal{E}, V, N)$ can be obtained from the integrated phase volume $\Omega=\Omega(\mathcal{E}, V, N)$ by performing a differentiation with respect to $\mathcal{E}$, i.e.,

$$
\begin{aligned}
\Omega & :=\int \mathrm{d} \Gamma_{N} \Theta\left(\mathcal{E}-H\left(\boldsymbol{x}_{1}, \ldots, \boldsymbol{p}_{N}\right)\right), \\
\omega & :=\int \mathrm{d} \Gamma_{N} \delta\left(\mathcal{E}-H\left(\boldsymbol{x}_{1}, \ldots, \boldsymbol{p}_{N}\right)\right)=\frac{\partial \Omega}{\partial \mathcal{E}},
\end{aligned}
$$

where, for identical particles, the integration measure in $N$-particle phase space is defined by

$$
\mathrm{d} \Gamma_{N}:=\left(N ! h^{d N}\right)^{-1} \begin{cases}\mathrm{~d}^{d} x_{1} \cdots \mathrm{d}^{d} x_{N} \mathrm{~d}^{d} p_{1} \cdots \mathrm{d}^{d} p_{N}, & \boldsymbol{x}_{s} \in \mathbb{V}, \\ 0, & \boldsymbol{x}_{s} \notin \mathbb{V} .\end{cases}
$$

\footnotetext{
54 The BGS entropy $\mathfrak{S}\left[\phi \mid h^{-d}\right]$ from Eq. (123a) is a relative entropy [487-491] with respect to the Lebesgue measure (normalized by the constant $h^{d}$ ) on the $2 d$-dimensional one-particle phase space $\{(\boldsymbol{x}, \boldsymbol{p})\}$; i.e., by writing Eq. (123) one has already fixed a specific reference measure $[475,491]$.

55 Alternative derivations of the Jüttner distribution are discussed by Pauli [123], Synge [188], Matolcsi et al. [227], and Debbasch [242].
} 

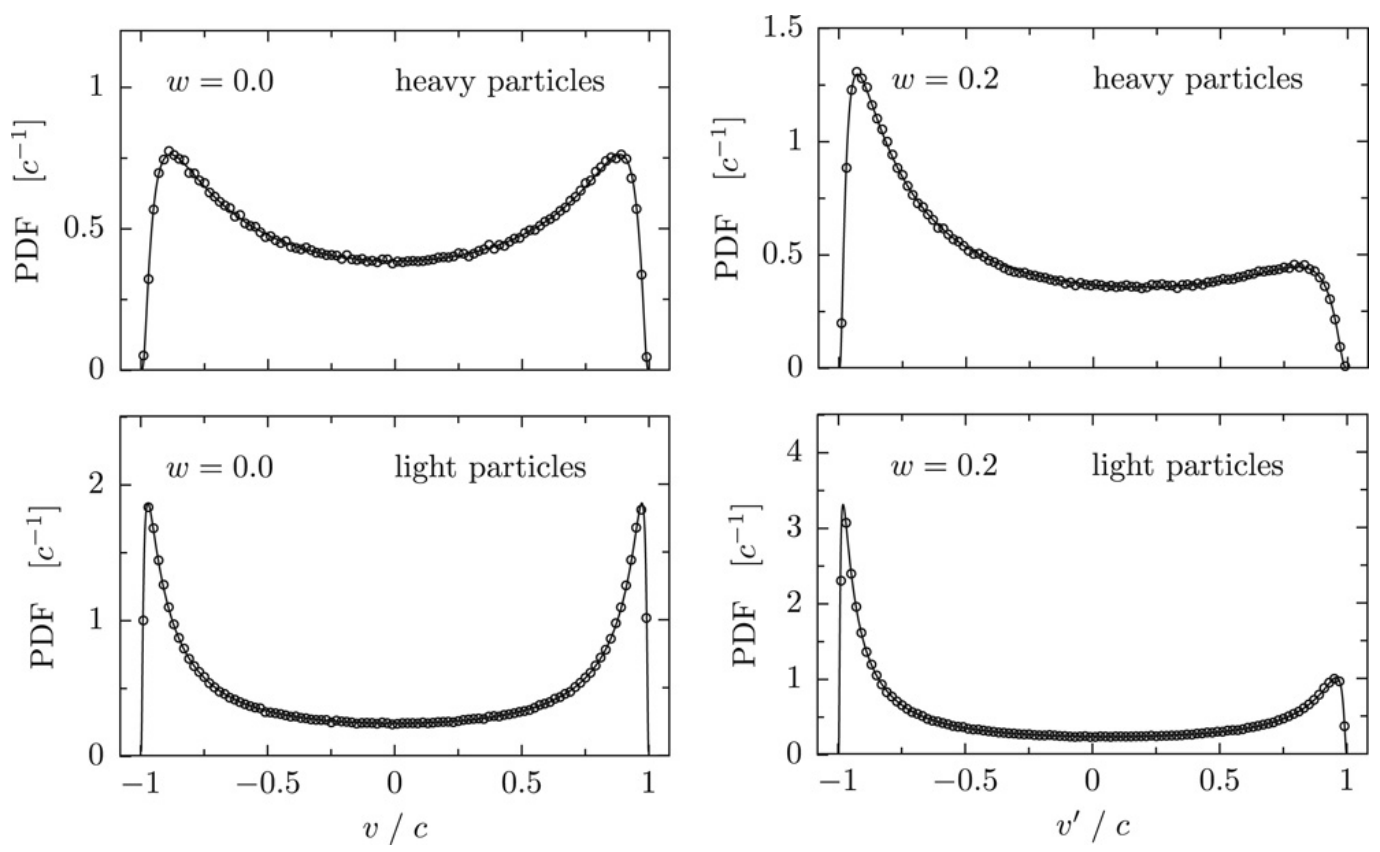

Fig. 4. Left column: Equilibrium velocity PDFs as measured by an observer with velocity $w=0$ in the lab frame $\Sigma$. The numerically obtained one-particle velocity PDFs $(O)$ are based on a fully relativistic, deterministic $1 \mathrm{D}$ molecular dynamics algorithm [202] with $N_{1}=1000$ light particles of mass $m_{1}$ and $N_{2}=1000$ heavy particles with mass $m_{2}=2 m_{1}$. Considering elastic interactions between the gas particles and elastic reflections at the boundaries, the mean energy per particle in the lab frame, $\bar{\epsilon}=\left[\sum_{r=1}^{N_{1}} m_{1} \gamma\left(v_{r}\right) v_{r}+\sum_{s=1}^{N_{2}} m_{2} \gamma\left(v_{s}\right) v_{s}\right] /\left(N_{1}+N_{2}\right)$, remains conserved during the simulation. The solid curves in the upper and lower panel correspond to Jüttner functions (118a) with different particle masses but same inverse temperature parameter $\beta=0.701\left(m_{1} c^{2}\right)^{-1}$. The latter can be determined from the value $\bar{\epsilon}=2.5 m_{1} c^{2}$ used in the simulation by the procedure described in [202]. Right column: Equilibrium velocity PDFs as measured by a moving observer $\Sigma^{\prime}$ who travels at constant velocity $w=0.2 c$ relative to the lab frame $\Sigma$. Parameter values and initial conditions are the same as those in the left column. In contrast to the diagrams with $w=0$, the PDFs for $w=0.2$ are based $\Sigma^{\prime}$-simultaneously measured velocities. The solid curves represent the theoretically expected, Lorentz-boosted Jüttner function $\psi_{\mathrm{J}}^{\prime}\left(v^{\prime} ; m, \beta, w\right)$ from Eq. (129). The very good agreement with the simulation data corroborates the validity of the Jüttner distribution. In particular, since in the stationary (equilibrium) state, both particle species are characterized by the same $\beta$-value, the definition $\mathcal{T}:=\left(k_{\mathrm{B}} \beta\right)^{-1}$ yields a meaningful statistical temperature concept.

The one-particle momentum PDF corresponding to $f_{N}^{\mathrm{MC}}$ from Eq. (124b) is defined by

$$
\begin{aligned}
\phi_{(N)}(\boldsymbol{p}) & :=\left\langle N^{-1} \sum_{s=1}^{N} \delta\left(\boldsymbol{p}-\boldsymbol{p}_{N}\right)\right\rangle^{\mathrm{MC}} \\
& =\int \mathrm{d} \Gamma_{N} f_{N}^{\mathrm{MC}}\left(\boldsymbol{x}_{1}, \ldots, \boldsymbol{p}_{N}\right) N^{-1} \sum_{s=1}^{N} \delta\left(\boldsymbol{p}-\boldsymbol{p}_{N}\right) .
\end{aligned}
$$

As discussed in Pauli's early review [123] and more recently also by Matolsci et al. [227], in the limit of an infinite particle number $N \rightarrow \infty$, the PDFs $\phi_{(N)}$ converge to the Jüttner distribution $\phi_{\mathrm{J}}$ from Eq. (118b).

Quite generally, a microcanonical PDF of the form Eq. (124b) gives rise to a number of rigorous equipartition theorems [287, 288,492 ] such as, e.g., ${ }^{56}$

$$
\left\langle p_{s}^{i} \frac{\partial H}{\partial p_{s}^{i}}\right\rangle^{\mathrm{MC}}=\frac{\Omega}{\omega}=\left[\frac{\partial}{\partial \mathcal{E}} \ln \Omega\right]^{-1}=: k_{\mathrm{B}} \mathcal{T}^{\mathrm{MC}}(\mathcal{E}, N),
$$

for each $s=1, \ldots, N$ and $i=1, \ldots, d$. The rhs of Eq. (126) defines [287,288,492] the usual microcanonical temperature of the gas in the lab frame. In the case of the Hamiltonian (124a), the equipartition theorem (126) is consistent with Eq. (122) upon identifying $\beta=\left(k_{\mathrm{B}} \mathcal{T}^{\mathrm{MC}}\right)^{-1}$ in the limit $N \gg 1$.

Computer experiments. Although the Jüttner distribution (118) became widely accepted [123,179,188,242,294,311], several modifications have been discussed in the literature [22,195,196,198,199,232,235,475]. The validity of Jüttner's (118) was recently confirmed in fully relativistic one-dimensional (1D) molecular dynamics simulations [202], see Fig. 4, as well as in (semi-)relativistic two and three-dimensional simulations [201,493]. By restricting the dynamics to one space dimension, it is possible simulate localized elastic particle interactions in a relativistically consistent manner without needing to introduce fields. This is due to the fact that, in the $1 \mathrm{D}$ case, the outgoing momenta $\left(\hat{p}_{A}, \hat{p}_{B}\right)$ of two colliding particles $A$ and $B$ are

56 In this case, the summation convention is abrogated on the lhs. of Eq. (126); i.e., Eq. (126) holds separately for each $s=1, \ldots, N$ and $i=1, \ldots, d$. 
uniquely determined by the momentum values $\left(p_{A}, p_{B}\right)$ before the collision, by means of the relativistic energy momentum conservation laws [22]

$$
\begin{aligned}
& \epsilon\left(m_{A}, p_{A}\right)+\epsilon\left(m_{B}, p_{B}\right)=\epsilon\left(m_{A}, \hat{p}_{A}\right)+\epsilon\left(m_{B}, \hat{p}_{B}\right), \\
& p_{A}+p_{B}=\hat{p}_{A}+\hat{p}_{B},
\end{aligned}
$$

where $\epsilon(m, p)=\left(m^{2}+p^{2}\right)^{1 / 2}$. In higher space dimensions, additional assumptions about the spatio-temporal structure of the collision processes need to be made [245,249-251,253,273]. In order to be fully consistent with the requirements of special relativity, relativistic interactions $d>1$ space dimensions must be formulated in terms of fields. Direct simulation of the field dynamics is numerically expensive and, therefore, practically infeasible in most cases. Alternatively, one can use simplified semi-relativistic models such as, for example, effective hard-sphere models where the interaction radius is defined with respect to the center-of-mass frame of the colliding particles. Generally, it can be expected that such simplified models yield satisfactory results in the low density regime [201], but they may lead to inconsistencies at high densities e.g., when three-body encounters become relevant.

In addition to (in)validating theoretically predicted distribution functions, numerical simulations can be useful for illustrating the meaning of concepts such as 'temperature' and 'thermal equilibrium' in special relativity. Fig. 4 shows results of a 1D relativistic gas simulation as described in [202]. The simulated gas consists of $N_{1}$ 'light' particles (mass $m_{1}$ ) and $N_{2}$ 'heavy' particles (mass $m_{2}>m_{1}$ ). Particle collisions in the gas are governed by the conservation laws (127) and collisions with the boundaries are elastic in the lab frame (i.e., $p \rightarrow-p$ in $\Sigma$ ). As evident from Fig. 4, the numerically obtained stationary velocity distributions are very well matched by 1D Jüttner functions (118) with different masses but same parameter $\beta$. Hence, the Jüttner distribution not only agrees with the numerical data, it also yields a well-defined concept of 'temperature' in the lab frame: Intuitively, the temperature $\mathcal{T}$ is thought to be an intensive quantity that equilibrates to a common value if two or more systems are brought into contact with each other (i.e., may exchange different forms of energy). In the example considered here, it is natural to regard the particle species as two different subsystems that may exchange energy via elastic collisions. After a certain relaxation time, the combined system approaches a thermodynamic equilibrium state', where each subsystem is described by the same asymptotic, two-parametric velocity PDF $\psi_{\mathrm{J}}\left(v ; m_{i}, \beta\right)$, differing only via the rest masses $m_{i}$. The commonly shared distribution parameter $\beta$ may thus be used to define a relativistic (statistical) equilibrium temperature in $\Sigma$ by

$$
\mathcal{T}:=\left(k_{\mathrm{B}} \beta\right)^{-1},
$$

in agreement with the interpretation of $\beta$ in the nonrelativistic case.

However, for this concept of temperature to be meaningful, a restriction of the accessible spatial volume is required - be it by means of periodic boundary conditions, by imposing reflecting walls or other types of confinements [157]. ${ }^{57}$ Otherwise, it cannot be expected that a many-particle system approaches a universal stationary equilibrium state independent of the specific initial conditions.

The simulations can also be used to determine the equilibrium velocity distributions as seen from another frame $\Sigma^{\prime}$, moving with velocity $w$ relative to the lab frame $\Sigma$, see Fig. 4 . In contrast to the diagrams for $w=0$, the numerical data points for $w=0.2$ are obtained by measuring the particle velocities $v_{s}^{\prime}$ simultaneously with respect to $\Sigma^{\prime}$. The solid curves in the right column of Fig. 4 correspond to the PDF

$$
\psi_{\mathrm{J}}^{\prime}\left(v^{\prime} ; m, \beta, w\right)=\gamma(w)^{-1} \frac{m \gamma\left(v^{\prime}\right)^{3}}{Z_{1}} \exp \left[-\beta \gamma(w) m \gamma\left(v^{\prime}\right)\left(1+w v^{\prime}\right)\right],
$$

obtained by making use of transformation law (114). The excellent agreement between the simulation data and Eq. (129) confirms the validity of Eq. (114) and, thereby, also the scalar Lorentz transformation behavior of the phase space PDF $f(t, \boldsymbol{x}, \boldsymbol{p})$ [151]. Moreover, for the model under consideration, one may state more precisely: Two relativistic gas components are in 'thermodynamic equilibrium' for any observer if their one-particle velocity PDFs are given by generalized Jüttner functions (129) with same parameters $\beta$ and $w$. It is only in this case that the net energy-momentum transfer between the different gas components in the container vanishes (a more general version of this criterion is discussed in Ref. [150]).

The numerical simulations illustrate that $\mathcal{T}=\left(k_{\mathrm{B}} \beta\right)^{-1}$ yields a useful statistical temperature concept in the lab frame $\Sigma$, defined by the boundary (container box). The question as to which temperature value is measured by a moving observer $\mathcal{O}^{\prime}$ cannot be uniquely answered - the answer depends on the respective definitions (i.e., measurement conventions) for heat, internal energy, etc. as employed by $\mathcal{O}^{\prime}$. These aspects are discussed in more detail in the Appendix C. At this point, it suffices to mention that $\mathcal{T}:=\left(k_{\mathrm{B}} \beta_{\mathrm{J}}\right)^{-1}$ can also be determined from $t^{\prime}$-simultaneously sampled velocity data (right column

\footnotetext{
57 The critical role of the boundary conditions in relativistic systems has been emphasized by Sinyukov [494] and van Kampen [150,151]. Loosely speaking, if a many-particle system has reached a universal stationary equilibrium state, then each particle 'knows' about the presence of the confinement/walls because equilibration typically requires momentum reversal at the walls, e.g., in order to maintain a uniform density and a well-balanced average collision frequency. In particular, if the walls are considered to be stationary objects then they single out a preferred frame of reference. The relevance of the boundary conditions is even more obvious in quantum mechanics/statistics due to their direct effect on the energy spectra and, thus, on the density of states [194].
} 

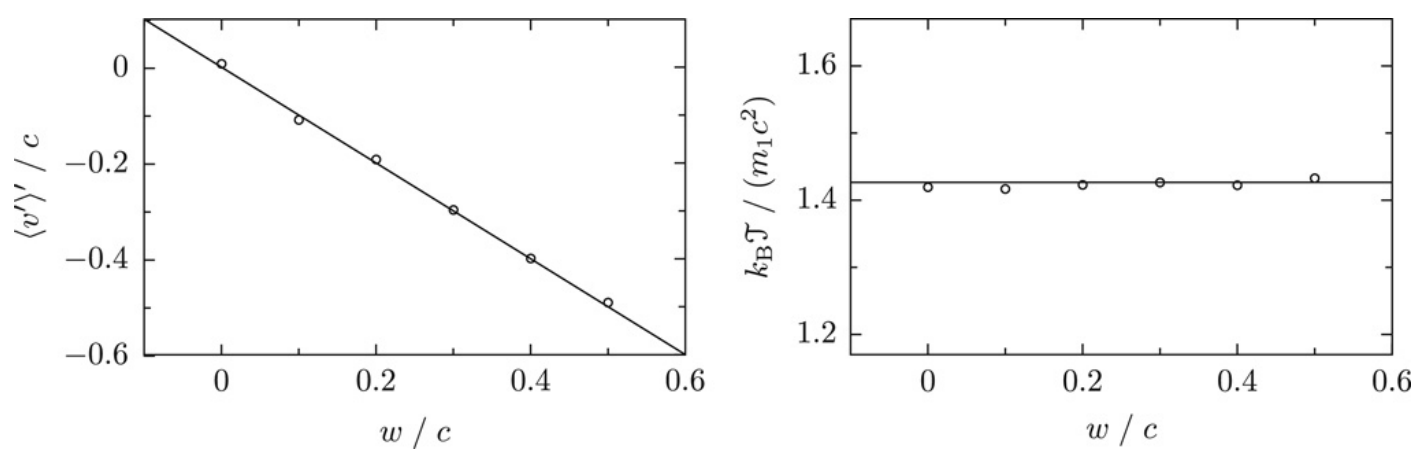

Fig. 5. Measured mean particle velocity $(\bigcirc$, left digram) and estimated temperature ( $\bigcirc$, right diagram) based on Eqs. (130) as a function of the observer velocity $w$. Solid lines correspond to the theoretically expected values, respectively, using the same simulation parameters and initial conditions as in Fig. 4 .

of Fig. 4) by means of the expectation value ${ }^{58}$ [141]

$$
k_{\mathrm{B}} \mathcal{T}=m \gamma(w)^{3}\left\langle\gamma\left(v^{\prime}\right)\left(v^{\prime}+w\right)^{2}\right\rangle_{t^{\prime}},
$$

where

$$
w=-\left\langle v^{\prime}\right\rangle_{t^{\prime}}
$$

is the mean velocity of the gas measured by the moving observer. One can test the validity of Eq. (130a) explicitly by using simulation data obtained for different values of $w$, see Fig. 5. Hence, in this sense, Eq. (130) defines a Lorentz invariant statistical gas thermometer - when adopting this thermometer a moving body appears neither hotter nor colder [202]. ${ }^{59}$

We conclude this part with another general remark: The above discussion has focused on the case where an 'ordinary' thermal equilibrium state is approached, corresponding to a stationary 'exponential' one-particle momentum distribution of the Jüttner type $[186,194]$. In general, one could also imagine non-equilibrium scenarios that give rise to (quasi-)stationary momentum distributions which differ from the Jüttner function (see, e.g, [197,232,233,235-237,239]). When attempting to develop a thermodynamic formalism for such non-standard distributions, the entropy functional needs to be modified appropriately [492]. However, our subsequent discussion of relativistic Langevin equations will concentrate on the case where the heat bath, which surrounds the Brownian particle, is described by a spatially homogeneous Jüttner function.

\section{Relativistic Brownian motion processes in phase space}

The present section describes the generalization of the Langevin theory of Brownian motions to the framework of special relativity [11-15,17,18,20-22,24-26,31-34,394,395,475,495]. More precisely, we will consider stochastic differential equations (SDEs) that describe Markov processes in relativistic one-particle phase space. ${ }^{60}$ Relativistic Langevin equations present a useful tool for modeling the dynamics of relativistic particles in a random environment. Examples include the analysis of thermalization effects in relativistic plasmas [318,319,329,421] and astrophysical systems [326,496]. Here, we would like to provide a comprehensive introduction to the underlying mathematical theory.

From a conceptual perspective, one can distinguish two complementary approaches towards modeling relativistic stochastic processes in one-particle phase space: The first approach is based on postulating evolution equations for the (transition) probability density of the stochastic process, as for example, integro-differential master-type equations or Fokker-Planck-type partial differential equations. Within a relativistic framework, the condition of subluminal particle propagation imposes stringent constraints on the structure of the evolution equations. These aspects were studied in the early pioneering works of Łopuszaǹski [330], Schay [332] and Hakim [338]. Alternatively, one may start by postulating SDEs as phenomenological model equations and subsequently derive the evolution equations for the associated (transition) probability densities. Here, we are going to pursue the latter route for mainly two reasons: We will be able to directly compare with the nonrelativistic case discussed in Section 2 and, more importantly, the physical origin of the stochastic dynamics - the interaction with a complex background medium - is 'less hidden' within the Langevin approach. ${ }^{61}$

According to our knowledge, Debbasch et al. [11] were the first to propose relativistic Langevin equations as models for relativistic Brownian motions. More precisely, these authors constructed a relativistic Ornstein-Uhlenbeck process (ROUP)

\footnotetext{
58 Equation (130a) is obtained by combining the microcanonical equipartition theorem for a Hamiltonian $H=\sum_{i=1}^{N_{1}} E\left(m_{1}, p_{i}\right)+\sum_{j=1}^{N_{2}} E\left(m_{2}, p_{j}\right)$ with the Lorentz invariance of the relativistic phase space $\operatorname{PDF} f$.

59 The mean value from Eq. (130a) can be used to measure the rest temperature, which plays a central role in van Kampen's [150] approach to relativistic thermodynamics. Evidently, upon multiplying Eq. (130a) by factors $\gamma(w)^{\alpha}, \alpha \neq 0$ one can construct thermometers that measure 'other' temperatures; e.g., $\alpha=-1$ would correspond to Planck's [121] formulation of relativistic thermodynamics and $\alpha=1$ to proposals made by Eddington [124] and Ott [127]. 60 A main motivation for considering processes $(\boldsymbol{X}(t), \boldsymbol{P}(t))$ rather than 'pure' position processes $\boldsymbol{X}(t)$ is that in the relativistic case, it is impossible to define nontrivial relativistic Markov processes $\boldsymbol{X}(t)$ in Minkowski spacetime; cf. Łopuszaǹski [330], Dudley (Theorem 11.3 in [333]), Hakim (Proposition 2 in [338]), and also the discussion in Section 5.

61 For a detailed analysis of the complementary approach we refer to Hakim [338].
} 
by postulating an additive Gaussian white noise force in the rest frame of the bath while adapting the friction such that the stationary momentum distribution is given by a Jüttner function. During the past decade, various properties of the ROUP were studied by Debbasch and Rivet [12], Barbachoux et al. [13,14], and Zygadło [20]. As will be illustrated below, the ROUP represents a special limit case of a larger class of relativistic Langevin models [15,19,26,31,32], which also includes the relativistic Brownian motion (RBM) process proposed in $[17,18]$. Processes belonging to this class share the common feature that their stationary momentum distribution is given by a Jüttner function, but they may exhibit a significantly different relaxation behavior, resulting, for example, in a different temperature dependence of the spatial diffusion constant [15].

The present section is structured as follows. Section 4.1 introduces the axiomatic Langevin approach, i.e., suitable SDEs and fluctuation-dissipation relations are postulated in order to provide a simplified model of the complex interaction between the Brownian particle and its environment (heat bath). Specific example processes will be analyzed in Section 4.2 . Section 4.2.2 discusses the temperature dependence of the asymptotic mean square displacement for three different relativistic Brownian motion processes [15,25]. It is demonstrated that the diffusion constant is sensitive to variations of the friction coefficients. This implies that measurements of the diffusion constant may reveal information about the underlying microscopic interactions. In Section 4.4 relativistic Brownian motion processes will be analyzed from the viewpoint of a moving observer. The relativistic generalization of the binary collision model from Section 2.2.2 is discussed in Section 4.5, representing a simple microscopic model for relativistic Brownian motions.

\subsection{Relativistic Langevin and Fokker-Planck equations}

When considering Langevin equations as models of Brownian motion, one implicitly assumes that it is possible and reasonable to separate the degrees of freedom of the Brownian particle from those of the environment (heat bath). Adopting this point of view, one can specify two distinguished frames of reference: the lab frame $\Sigma$, defined as the inertial rest-frame of the heat bath, and the inertial frame $\Sigma_{*}$ that is comoving with the Brownian particle at a given instant of time. ${ }^{62}$ To start with, we consider the question how one can model the dynamics of a relativistic Brownian particle in the lab frame $\Sigma$.

\subsubsection{Relativistic Langevin equations: General construction principles}

Roughly speaking, a relativistic Brownian motion process is a stochastic process whose absolute velocity $|\boldsymbol{V}(t)|=$ $|\mathrm{d} \boldsymbol{X} / \mathrm{d} t|$ does not exceed the light speed $c=1$ at any time; i.e., the process must satisfy the condition

$$
|\boldsymbol{V}(t)|=\frac{|\boldsymbol{P}|}{P^{0}}=\frac{|\boldsymbol{P}|}{\left(M^{2}+\boldsymbol{P}^{2}\right)^{1 / 2}} \leq 1 \quad \forall t
$$

where $M>0$ denotes the rest mass of the Brownian particle. Of particular interest here are processes that can be modeled by means of SDEs similar to the nonrelativistic Langevin equations (5). The basic idea for constructing such processes is to couple the noise sources (e.g., Wiener processes) only to the relativistic momentum $\boldsymbol{P}=\left(P^{i}\right), i=1, \ldots, d$, which can take values in whole $\mathbb{R}^{d}$. By doing so, the condition (131) is automatically fulfilled. However, before one can actually write down specific SDEs for the relativistic momentum components $P^{i}$, an additional question needs to be addressed, namely $[30,338,497]$ :

The choice of the time parameter. A fundamental assumption (postulate) of nonrelativistic Galilean physics is the existence of a universal time $t$. Therefore, within the nonrelativistic Langevin theory, it is quite natural to identify this universal time $t$ with the time parameter of the stochastic driving process, which is often taken to be a multi-dimensional Wiener process $\boldsymbol{B}(t)$. By contrast, in special relativity, the notion of time becomes frame-dependent and it is important to specify in advance which time parameter is used to quantify the fluctuations of the stochastic driving process.

When considering the stochastic motion of a relativistic Brownian particle, two characteristic time parameters can be distinguished: The coordinate time $t$ of the inertial lab frame $\Sigma$, which may be interpreted as the proper time of the heat bath, and the proper time $\tau$ of the Brownian particle. In principle, either of the two parameters could be used to formulate SDEs for the spatial components of the particle momentum, $\boldsymbol{P}=\left(P^{i}\right)$. Within the conventional Langevin picture of Brownian motion, one usually considers friction and noise as externally imposed forces that act upon the Brownian particle and reflect the fluctuations within the heat bath. Therefore, it seems more natural to characterize the statistical properties of the noise source in terms of the lab time $t$. However, as we shall discuss in Section 4.3 one can reparameterize a given lab-time Langevin equation in term of the associated proper-time. ${ }^{63}$

Lab-time Langevin equation. Within the lab-time approach [11-14,17,18,20,394,498] one aims at constructing $t$ parameterized $2 d$-dimensional stochastic processes $\{\boldsymbol{X}(t), \boldsymbol{P}(t)\}$ with respect to the lab frame $\Sigma$, where the position

\footnotetext{
62 Apart from an irrelevant shift of the origin, the inertial lab frame $\Sigma$ is uniquely determined by the requirement that the mean velocity of the heat bath particles, which is assumed to be constant in any inertial frame, vanishes in $\Sigma$. Similarly, the instantaneously comoving frame $\Sigma_{*}$ is determined by the condition that the $\Sigma_{*}$-velocity of the Brownian particle is equal to zero at the given instant of time. Generally, we assume that the time coordinates $t$ and $t_{*}$ can be measured, e.g., by using atomic clocks that are at rest in $\Sigma$ or $\Sigma_{*}$, respectively.

63 Within this lab-time approach, the proper time becomes a stochastic quantity, and one could, for example, ask for the probability of finding at lab time $t$ the particle's proper time in the interval $[\tau, \tau+\mathrm{d} \tau]$. Conversely, if adopting the proper time $\tau$ as the primary deterministic evolution parameter, one could ask for the probability to find the particle at proper time $\tau$ within the spacetime interval $[t, t+\mathrm{d} t] \times[\boldsymbol{x}, \boldsymbol{x}+\mathrm{d} \boldsymbol{x}]$ with respect to the lab frame. The proper-time approach was studied in Refs. [337,338,391] and most recently in [30].
} 
coordinates $\boldsymbol{X}=\left(X^{i}\right)$ and the spatial momentum coordinates $\boldsymbol{P}=\left(P^{i}\right)$ are connected by the standard relativistic differential relation

$$
\mathrm{d} X^{i}(t)=\left(P^{i} / P^{0}\right) \mathrm{d} t
$$

with $P^{0}(t)=E(t)=\left(M^{2}+\boldsymbol{P}^{2}\right)^{1 / 2}$ denoting the relativistic energy of the particle and $V^{i}(t)=P^{i} / P^{0}$ its velocity components in $\Sigma$. Stochasticity is implemented into the dynamics by coupling the momentum components $P^{i}(t)$ to an external noise source via the $\mathrm{SDES}^{64}$

$$
\mathrm{d} P^{i}(t)=\mathcal{F}^{i} \mathrm{~d} t-a_{j}^{i} P^{j} \mathrm{~d} t+c^{i} \odot{ }_{j} \odot B^{j}(t),
$$

where

- $\mathcal{F}^{i}$ denotes a deterministic external force (e.g., Lorentz force), $-a_{j}^{i} P^{j}$ is a phenomenological friction force, and the last term represents noise;

- $\odot \in\{*, \circ, \bullet\}$ signals the discretization rule (i.e., the stochastic integral definition, cf. Appendix A);

- in general, the functions $\mathcal{F}^{i}, a_{j}^{i}$ and $c_{j}^{i}$ can depend on $(t, \boldsymbol{X}, \boldsymbol{P})$;

- the noise source is usually modeled by the $d$-dimensional standard Wiener ${ }^{65}$ process $\boldsymbol{B}(t)=\left(B^{1}(t), \ldots, B^{d}(t)\right)$ in a relativistic context.

Equations (132) govern the spatial components of the four-vectors $\left(X^{0}, \boldsymbol{X}\right)$ and $\left(P^{0}, \boldsymbol{P}\right)$. One can still add an equation for time component $X^{0}$ by setting

$$
\mathrm{d} X^{0}(t)=\left(P^{0} / P^{0}\right) \mathrm{d} t=\mathrm{d} t .
$$

The equation for the energy component $P^{0}$ can be derived from Eq. (132b) by applying the (backward) Ito formula, cf. Appendix A, to the mass-shell condition $P^{0}(t)=\left(M^{2}+\boldsymbol{P}^{2}\right)^{1 / 2}$, yielding

$$
\mathrm{d} P^{0}(t)=\left\{\left(\mathcal{F}_{i}-a_{i j} P^{j}\right) \frac{P^{i}}{P^{0}}+\lambda_{\odot} \frac{D_{i j}}{2}\left[\frac{\delta^{i j}}{P^{0}}-\frac{P^{i} P^{j}}{\left(P^{0}\right)^{3}}\right]\right\} \mathrm{d} t+\frac{P^{i}}{P^{0}} c_{i r} \odot \mathrm{d} B^{r}(t),
$$

where

$$
\mathcal{F}_{i}:=\mathcal{F}^{i}, \quad D_{i j}:=D^{i j}:=c^{i}{ }_{r} c^{j}, \quad c_{i r}:=c^{i}{ }_{r},
$$

and, depending on the discretization rule,

$$
\lambda_{*}=1, \quad \lambda_{\circ}=0, \quad \lambda_{\bullet}=-1 .
$$

The $\lambda_{\odot}$-term in Eq. (133b) reflects the modification of differential calculus for Ito and backward-Ito SDEs, cf. Appendix A.

Finally, we still note that the proper-time process associated with Eq. (132) is defined by

$$
\mathrm{d} \tau(t)=\left(M / P^{0}\right) \mathrm{d} t .
$$

In Section 4.3, we shall discuss how the lab-time Langevin equations (132) can be reparameterized in terms of $\tau$.

\subsubsection{Fokker-Planck equations}

In the case of the Ito rule " $\odot=*$ " in Eq. (132b), the corresponding Fokker-Planck equation (FPE) is given by [cf. Eq. (A.29)]

$$
\left(\frac{\partial}{\partial t}+\frac{p^{i}}{p^{0}} \frac{\partial}{\partial x^{i}}\right) f_{*}=\frac{\partial}{\partial p^{i}}\left[\left(-\mathcal{F}^{i}+a_{j}^{i} p^{j}\right) f_{*}+\frac{1}{2} \frac{\partial}{\partial p^{k}}\left(c_{r}^{i} c^{k}{ }_{r} f_{*}\right)\right],
$$

in the case of the Stratonovich-Fisk mid-point rule " $\odot=0$ " by

$$
\left(\frac{\partial}{\partial t}+\frac{p^{i}}{p^{0}} \frac{\partial}{\partial x^{i}}\right) f_{\circ}=\frac{\partial}{\partial p^{i}}\left[\left(-\mathcal{F}^{i}+a_{j}^{i} p^{j}-\frac{1}{2} c^{k}{ }_{r} \frac{\partial}{\partial p^{k}}{ }^{i}{ }_{r}\right) f_{\circ}+\frac{1}{2} \frac{\partial}{\partial p^{k}}\left(c^{i}{ }_{r} c^{k}{ }_{r} f_{\circ}\right)\right]
$$

and in the case of the post-point (backward Ito) rule " $\odot=\bullet$ " by

$$
\left(\frac{\partial}{\partial t}+\frac{p^{i}}{p^{0}} \frac{\partial}{\partial x^{i}}\right) f_{\bullet}=\frac{\partial}{\partial p^{i}}\left[\left(-\mathcal{F}^{i}+a_{j}^{i} p^{j}-c^{k}{ }_{r} \frac{\partial}{\partial p^{k}} c_{r}^{i}\right) f_{\bullet}+\frac{1}{2} \frac{\partial}{\partial p^{k}}\left(c_{r}^{i} c^{k}{ }_{r} f_{\bullet}\right)\right]
$$

with $f_{\odot}(t, \boldsymbol{x}, \boldsymbol{p})$ denoting the phase space PDF of the relativistic Brownian particle in $\Sigma$, and $p^{0}=\left(M^{2}+\boldsymbol{p}^{2}\right)^{1 / 2}$, respectively.

Deterministic initial data $\boldsymbol{X}(0)=\boldsymbol{x}_{0}$ and $\boldsymbol{P}(0)=\boldsymbol{p}_{0}$ corresponds to the localized initial condition

$$
f_{\odot}(0, \boldsymbol{x}, \boldsymbol{p})=\delta\left(\boldsymbol{x}-\boldsymbol{x}_{0}\right) \delta\left(\boldsymbol{p}-\boldsymbol{p}_{0}\right) .
$$

Due to the linearity of Eq. (135), more general solutions can be obtained by integrating the solutions of Eqs. (135) over a nonlocalized initial distribution $f_{0}\left(\boldsymbol{x}_{0}, \boldsymbol{p}_{0}\right)$. However, it should be noted that, within a relativistic framework, it is very difficult to determine non-localized lab-isochronous initial data by means of experimental measurements.

\footnotetext{
64 E.g., for the post-point rule " $\odot=\bullet$ ", Eqs. (132) constitute a special case of the general SDE (A.28), upon identifying $n=2 d, \boldsymbol{Y}=$ $\left(X^{1}, \ldots, X^{d}, P^{1}, \ldots, P^{d}\right)$ and $A^{i}=P^{i} / P^{0}, C^{i}{ }_{j}=0, A^{i+d}=-a^{i}{ }_{j} P^{j}, C^{i+d}{ }_{j}=c^{i}{ }_{j}$ for $i=1, \ldots, d$.

65 In principle, one could also consider other driving process (as, e.g., Levy or Poisson processes) but we are not aware of such studies.
} 


\subsubsection{Free motions in an isotropic bath and Einstein relations}

Similar to the nonrelativistic case, physical constraints on the coefficient functions $a_{j}^{i}$ and $c_{j}^{i}$ come from symmetry properties of the heat bath and from the requirement that Eqs. (132) must reproduce the correct stationary distribution and the correct relaxation behavior. For example, in the absence of external force-fields, $\mathcal{F}^{i} \equiv 0$, and if the heat bath is stationary, isotropic and homogeneous in the lab frame $\Sigma$, the coefficient matrices take the simplified diagonal form

$$
a_{j}^{i}=\alpha \delta_{j}^{i}, \quad c_{j}^{i}=(2 D)^{1 / 2} \delta_{j}^{i} .
$$

Under the stated assumptions, the functions $\alpha$ and $D$ depend only on the Brownian particles' absolute momentum - or, equivalently, on its relativistic energy $p^{0}=\left(M^{2}+\boldsymbol{p}^{2}\right)^{1 / 2}$ i.e., $\alpha(\boldsymbol{p})=\hat{\alpha}\left(p^{0}\right)$ and $D(\boldsymbol{p})=\hat{D}\left(p^{0}\right)$. In this case, the relativistic Langevin equations (132) simplify to

$$
\begin{aligned}
& \mathrm{d} X^{i}(t)=\left(P^{i} / P^{0}\right) \mathrm{d} t, \\
& \mathrm{~d} P^{i}(t)=-\hat{\alpha} P^{i} \mathrm{~d} t+(2 \hat{D})^{1 / 2} \odot \mathrm{d} B^{i}(t),
\end{aligned}
$$

and the Fokker-Planck equations (135) take the form

$$
\begin{aligned}
& \left(\frac{\partial}{\partial t}+\frac{p^{i}}{p^{0}} \frac{\partial}{\partial x^{i}}\right) f_{*}=\frac{\partial}{\partial p^{i}}\left[\hat{\alpha} p^{i} f_{*}+\frac{\partial}{\partial p^{i}}\left(\hat{D} f_{*}\right)\right], \\
& \left(\frac{\partial}{\partial t}+\frac{p^{i}}{p^{0}} \frac{\partial}{\partial x^{i}}\right) f_{\circ}=\frac{\partial}{\partial p^{i}}\left[\hat{\alpha} p^{i} f_{\circ}+\hat{D}^{1 / 2} \frac{\partial}{\partial p^{i}}\left(\hat{D}^{1 / 2} f_{\circ}\right)\right], \\
& \left(\frac{\partial}{\partial t}+\frac{p^{i}}{p^{0}} \frac{\partial}{\partial x^{i}}\right) f_{\bullet}=\frac{\partial}{\partial p^{i}}\left[\hat{\alpha} p^{i} f_{\bullet}+\hat{D} \frac{\partial}{\partial p^{i}} f_{\bullet}\right] .
\end{aligned}
$$

An additional constraint on the functions $\alpha(p)=\hat{\alpha}\left(p^{0}\right)$ and $D(p)=\hat{D}\left(p^{0}\right)$ arises from thermostatistical considerations: If the motion of the Brownian particle is restricted to a finite volume $\mathbb{V} \subset \mathbb{R}^{d}$ and if the heat bath is in a thermal equilibrium state at temperature $\mathcal{T}=\left(k_{\mathrm{B}} \beta\right)^{-1}$, then the expected stationary solution $f_{\infty}(\boldsymbol{x}, \boldsymbol{p})$ of Eq. (135) is a spatially homogeneous Jüttner distribution [186], 66

$$
f_{\infty}(\boldsymbol{x}, \boldsymbol{p})=\mathcal{N} \exp \left[-\beta\left(M^{2}+\boldsymbol{p}^{2}\right)^{1 / 2}\right] \ell(\boldsymbol{x} ; \mathbb{V}),
$$

with $\ell(\boldsymbol{x} ; \mathbb{V})$ being the indicator function of the accessible volume $\mathbb{V}$ as defined in Eq. (103b). By inserting Eq. (139) into the Fokker-Planck equations (135) one finds that, depending on the discretization rule, the functions $\alpha(p)=\hat{\alpha}\left(p^{0}\right)$ and $D(p)=\hat{D}\left(p^{0}\right)$ must satisfy the generalized fluctuation-dissipation relations $[11,17,18]$

$$
\begin{array}{ll}
\odot=*: & 0 \equiv \hat{\alpha}\left(p^{0}\right) p^{0}-\beta \hat{D}\left(p^{0}\right)+\hat{D}^{\prime}\left(p^{0}\right), \\
\odot=\circ: & 0 \equiv \hat{\alpha}\left(p^{0}\right) p^{0}-\beta \hat{D}\left(p^{0}\right)+\hat{D}^{\prime}\left(p^{0}\right) / 2, \\
\odot=\bullet: & 0 \equiv \hat{\alpha}\left(p^{0}\right) p^{0}-\beta \hat{D}\left(p^{0}\right),
\end{array}
$$

where $\hat{D}^{\prime}\left(p^{0}\right):=\mathrm{d} \hat{D}\left(p^{0}\right) / \mathrm{d} p^{0}$. Equations (140) are also referred to as the relativistic Einstein relations. In particular, by comparing Eq. (140c) with the nonrelativistic Einstein relation (20), we note that the mass has been replaced with energy $p^{0}$ in the relativistic case.

\subsection{One-dimensional examples and mean square displacement}

In this part, we will consider one-dimensional example processes $(d=1)$. Their generalization to higher space dimensions is straightforward but analytic calculations become more tedious. We first summarize the SDEs for the energy process $P^{0}(t)$ and the velocity process $V(t):=P(t) / P^{0}(t)$ in Section 4.2.1. Subsequently, analytical and numerical results for the asymptotic diffusion constants of specific example processes will be discussed $[15,25]$.

\subsubsection{Discretization rules, energy and velocity equations}

Considering an isotropic thermalized heat bath in $d=1$ space dimensions and the post-point discretization, Eq. (137) takes form

$$
\begin{aligned}
& \mathrm{d} X(t)=\left(P / P^{0}\right) \mathrm{d} t, \\
& \mathrm{~d} P(t)=-\alpha_{\bullet}(P) P \mathrm{~d} t+[2 D(P)]^{1 / 2} \bullet \mathrm{d} B(t),
\end{aligned}
$$

where $B(t)$ is characterized by the Gaussian distribution $(5 \mathrm{~d})$ and $\alpha_{\bullet}(p)=\hat{\alpha}_{\bullet}\left(p^{0}\right)$ and $D(p)=\hat{D}\left(p^{0}\right)$. Similar to the nonrelativistic case, one can replace Eq. (141a) by the equivalent Stratonovich-Fisk SDE ${ }^{67}$

66 Cf. the discussion in Section 3.2.2.

67 See also the corresponding discussion by Hänggi [499], and Hänggi and Thomas (page 293 of Ref. [77]). 


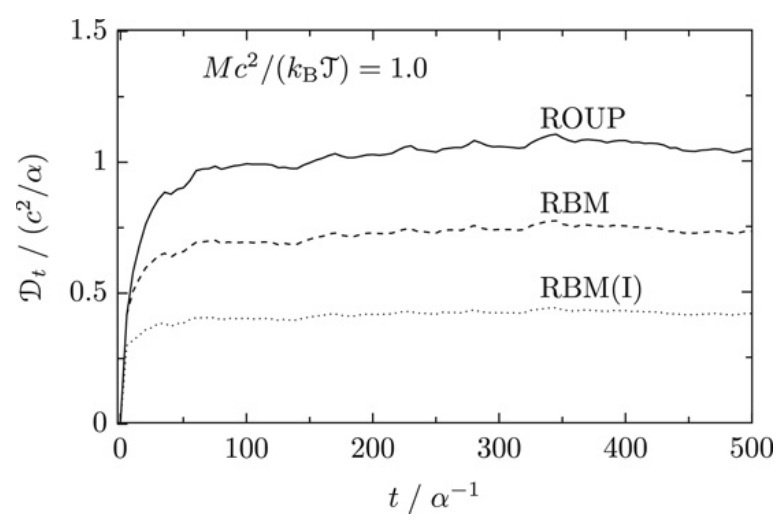

Fig. 6. Time evolution of the spatial mean square displacement $\mathcal{D}_{t}:=\left\langle[X(t)-X(0)]^{2}\right\rangle /(2 t)$ for the ROUP [11] model (solid line) from Eq. (151d), the RBM [17] model (dashed) from Eq. (155b) and the RBM(I) model (dotted) from Eq. (159a) at same temperature-mass ratio $\chi^{-1}:=k_{\mathrm{B}} \mathcal{T} /\left(M c^{2}\right)=1$. The plots are based on a simulation with $N=1000$ trajectories, initial conditions $X(0)=0, P(0)=0$ for each trajectory, and discretization time step $\Delta t=10^{-4} \alpha_{c / \uparrow}^{-1}$.

$$
\begin{aligned}
& \mathrm{d} P(t)=-\alpha_{\circ}(P) P \mathrm{~d} t+[2 D(P)]^{1 / 2} \circ \mathrm{d} B(t) \\
& \alpha_{\circ}(p):=\alpha_{\bullet}(p)-D^{\prime}(p) /(2 p),
\end{aligned}
$$

or by the equivalent Ito SDE

$$
\begin{aligned}
& \mathrm{d} P(t)=-\alpha_{*}(P) P \mathrm{~d} t+[2 D(P)]^{1 / 2} * \mathrm{~d} B(t) \\
& \alpha_{*}(p):=\alpha_{\bullet}(p)-D^{\prime}(p) / p,
\end{aligned}
$$

where $D^{\prime}(p):=\mathrm{d} D(p) / \mathrm{d} p$. Compared with Eqs. (141a) and (141b), the Ito form (141c) is most convenient for numerical simulations.

Imposing that the solution of corresponding FPE (138) be given by a one-dimensional Jüttner function of the form (139), the friction and noise coefficients must satisfy the Einstein relations (140). In terms of the friction coefficients $\alpha_{\odot}(p)$, the Einstein relations can also be rewritten as

$$
\begin{aligned}
& 0 \equiv \alpha_{\bullet}(p) p^{0}-\beta D(p) \\
& 0 \equiv \alpha_{\circ}(p) p^{0}-\beta D(p)+D^{\prime}(p) p^{0} /(2 p) \\
& 0 \equiv \alpha_{*}(p) p^{0}-\beta D(p)+D^{\prime}(p) p^{0} / p .
\end{aligned}
$$

From Eq. (133b) one obtains for the relativistic energy process $P^{0}(t):=\left(M^{2}+P^{2}\right)^{1 / 2}$ the following SDE

$$
\begin{aligned}
\mathrm{d} P^{0}(t)= & \left\{-\hat{\alpha}_{\odot}\left(P^{0}\right) P^{0}\left[1-\left(\frac{M}{P^{0}}\right)^{2}\right]+\lambda_{\odot} \frac{\hat{D}\left(P^{0}\right)}{P^{0}}\left(\frac{M}{P^{0}}\right)^{2}\right\} \mathrm{d} t \\
& +\left\{2 \hat{D}\left(P^{0}\right)\left[1-\left(\frac{M}{P^{0}}\right)^{2}\right]\right\}^{1 / 2} \odot \mathrm{d} B(t),
\end{aligned}
$$

where $\lambda_{*}=1, \lambda_{\circ}=0$, and $\lambda_{\bullet}=-1$. Furthermore, by defining for $P(V)=M V\left(1-V^{2}\right)^{-1 / 2}$ new coefficients

$$
\tilde{\alpha}(V):=\alpha(P(V)), \quad \tilde{D}(V):=D(P(V))
$$

and applying the (backward) Ito formula, cf. Appendix A, to the relativistic velocity formula $V(t)=P /\left(M^{2}+\boldsymbol{P}^{2}\right)^{1 / 2}$, one finds the following SDE for the velocity process

$$
\mathrm{d} V(t)=\left[-\tilde{\alpha}(V)\left(1-V^{2}\right)-\lambda_{\odot}\left(\frac{3 \tilde{D}}{M^{2}}\right)\left(1-V^{2}\right)^{2}\right] V \mathrm{~d} t+\left[\left(\frac{2 \tilde{D}}{M^{2}}\right)\left(1-V^{2}\right)^{3}\right]^{1 / 2} \odot \mathrm{d} B(t) .
$$

As discussed in the next section, this equation can be used to calculate the asymptotic mean square displacement.

\subsubsection{Asymptotic mean square displacement}

A primary objective within any Brownian theory is to determine the asymptotic diffusion constant $\mathscr{D}_{\infty}$, corresponding to the plateau values in Fig. 6. For a one-dimensional diffusion process $X(t)$ with velocity $V(t)$, the asymptotic diffusion constant $\mathcal{D}_{\infty}$ is defined by

$$
D_{\infty}=\lim _{t \rightarrow \infty}\left\langle[X(t)-X(0)]^{2}\right\rangle /(2 t),
$$


where the spatial displacement is given by

$$
X(t)-X(0)=\int_{0}^{t} \mathrm{~d} s V(s) .
$$

The asymptotic diffusion constant $\mathscr{D}_{\infty}$ may be expressed in terms of the velocity correlation function $\langle V(t) V(s)\rangle$ by virtue of

$$
\begin{aligned}
\mathscr{D}_{\infty} & =\lim _{t \rightarrow \infty} \frac{1}{2} \frac{\mathrm{d}}{\mathrm{d} t}\left\langle[X(t)-X(0)]^{2}\right\rangle \\
& =\lim _{t \rightarrow \infty} \frac{1}{2} \frac{\mathrm{d}}{\mathrm{d} t} \int_{0}^{t} \mathrm{~d} s \int_{0}^{t} \mathrm{~d} s^{\prime}\left\langle V(s) V\left(s^{\prime}\right)\right\rangle \\
& =\lim _{t \rightarrow \infty} \int_{0}^{t} \mathrm{~d} s\langle V(t) V(s)\rangle .
\end{aligned}
$$

Assuming that the velocity process $V(t)$ is (approximately) stationary, which means that $\langle V(t) V(s)\rangle=\langle V(t-s) V(0)\rangle$ holds (at least in good approximation), and substituting $u=t-s$, we recover Kubo's formula

$$
\mathscr{D}_{\infty}=\lim _{t \rightarrow \infty} \int_{0}^{t} \mathrm{~d} u\langle V(u) V(0)\rangle .
$$

As recently discussed by Lindner [25], for a one-dimensional Langevin equation of the form

$$
\mathrm{d} V(t)=-a_{\bullet}(V) V \mathrm{~d} t+[2 b(V)]^{1 / 2} \bullet \mathrm{d} B(t)
$$

with symmetric coefficient functions, $a_{\bullet}(v)=a_{\bullet}(-v)$ and $b(v)=b(-v)$, the Kubo formula (147) gives rise to the following integral representation for the asymptotic diffusion constant:

$$
D_{\infty}=\frac{\int_{0}^{v_{+}} \mathrm{d} y \mathrm{e}^{U(y)}\left[\int_{y}^{v_{+}} \mathrm{d} x \mathrm{e}^{-U(x)} x / b(x)\right]^{2}}{\int_{0}^{v_{+}} \mathrm{d} z \mathrm{e}^{-U(z)} / b(z)} .
$$

Here, $v_{+} \in[0, \infty]$ represents the upper bound for the velocity range, and

$$
U(v):=\int_{0}^{v} \mathrm{~d} w \mu_{*}(w) / b(w)
$$

is an effective velocity potential with Ito drift

$$
\mu_{*}(v):=a_{*}(v) v=a_{\bullet}(v) v-b^{\prime}(v) .
$$

In general, the formula (149) has to be integrated numerically, but for the first two models from Section 4.2 .3 the integrals may also be evaluated analytically. The $d$-dimensional generalization of Eq. (149) was recently derived by Angst and Franchi [15].

\subsubsection{Examples}

We discuss three specific one-dimensional relativistic Langevin models whose stationary momentum distributions are Jüttner functions $\phi_{\mathrm{J}}(p) \propto \exp \left[-\beta\left(p^{2}+M^{2}\right)^{1 / 2}\right]$ with heat bath temperature $\mathcal{T}=\left(k_{\mathrm{B}} \beta\right)^{-1}$. In this case, the relativistic Einstein relation (140) implies that only one of the two functions $\alpha_{\bullet}(p)$ and $D(p)$ can be chosen arbitrarily.

Constant noise amplitude. As a first example, we consider the so-called 'Relativistic Ornstein-Uhlenbeck process' (ROUP), proposed by Debbasch et al. [11,12] and also studied by Zygadło [20]. The ROUP is defined by the choice

$$
\alpha_{\bullet}(p)=\alpha_{c} M / p^{0},
$$

where $\alpha_{c}>0$ is a constant friction parameter. From the relativistic Einstein relation (140), one then finds

$$
D(p) \equiv \alpha_{\bullet}(p) p^{0} \beta^{-1}=\alpha_{c} M \beta^{-1}=: D_{c},
$$

i.e., the ROUP corresponds to the limit case of constant noise amplitude. The associated Langevin equation reads

$$
\begin{aligned}
\mathrm{d} P(t) & =-\alpha_{c} \frac{M}{P^{0}} P \mathrm{~d} t+\left(\frac{2 \alpha_{c} M}{\beta}\right)^{1 / 2} \bullet \mathrm{d} B(t) \\
& =-\alpha_{c} \frac{M}{P^{0}} P \mathrm{~d} t+\left(\frac{2 \alpha_{c} M}{\beta}\right)^{1 / 2} * \mathrm{~d} B(t)
\end{aligned}
$$


The discretization rule is irrelevant here, because the noise amplitude $D_{c}=\alpha_{c} M / \beta$ does not depend on the momentum $P$ for this particular case. However, the rules of stochastic calculus have to be specified, if one wishes to write down the SDE for the associated velocity process $V(t):=P / P^{0}$. Defining the useful abbreviation

$$
\chi:=\beta M=M /\left(k_{\mathrm{B}} \mathcal{T}\right),
$$

one finds

$$
\begin{aligned}
\mathrm{d} V(t) & =-\alpha_{c}\left[\left(1-V^{2}\right)^{3 / 2}-\frac{3}{\chi}\left(1-V^{2}\right)^{2}\right] V \mathrm{~d} t+\left[\frac{2 \alpha_{c}}{\chi}\left(1-V^{2}\right)^{3}\right]^{1 / 2} \bullet \mathrm{d} B(t) \\
& =-\alpha_{c}\left[\left(1-V^{2}\right)^{3 / 2}+\frac{3}{\chi}\left(1-V^{2}\right)^{2}\right] V \mathrm{~d} t+\left[\frac{2 \alpha_{c}}{\chi}\left(1-V^{2}\right)^{3}\right]^{1 / 2} * \mathrm{~d} B(t) .
\end{aligned}
$$

Using the Ito form, as required in Eq. (150b), we see that the ROUP (151d) corresponds to

$$
\begin{aligned}
& \mu_{*}(v)=\alpha_{c}\left[\left(1-v^{2}\right)^{3 / 2}+\frac{3}{\chi}\left(1-v^{2}\right)^{2}\right] v, \\
& b(v)=\frac{\alpha_{c}}{\chi}\left(1-v^{2}\right)^{3}
\end{aligned}
$$

with an upper velocity bound $v_{+}=c=1$ in Eq. (149). In this case, the general integral formula (149) for the asymptotic diffusion constant can be evaluated analytically by making use of the identity (153)

$$
(-1)^{v} \frac{\mathrm{d}^{\nu}}{\mathrm{d} \chi^{\nu}} K_{0}(\chi)=\int_{0}^{1} \mathrm{~d} v \exp \left(-\frac{\chi}{\sqrt{1-v^{2}}}\right)\left(1-v^{2}\right)^{-(v+2) / 2}
$$

where, for $v=0,1,2 \ldots, K_{v}(z)$ denotes the modified Bessel function of the second kind [486]. Remarkably, one recovers for the ROUP the 'classical' result [15], cf. Eq. (33),

$$
\mathcal{D}_{\infty}^{\text {ROUP }}=\left(\alpha_{c} \chi\right)^{-1}=k_{\mathrm{B}} \mathcal{T} /\left(M \alpha_{c}\right)
$$

for all parameter values $\left(\alpha_{c}, \mathcal{T}, M\right)$.

Constant friction coefficient in the backward-Ito SDE. An alternative relativistic Brownian motion (RBM) model [17,18] corresponds to the special case of a constant friction function $\alpha_{\bullet}(p) \equiv \alpha_{\dagger}$ in the backward-Ito SDE (141). In this case, the relativistic Einstein relation (140) yields the momentum dependent noise amplitude

$$
D(p)=\alpha_{\dagger} p^{0} \beta^{-1} .
$$

The relativistic (backward) Ito Langevin equations of this model read explicitly

$$
\begin{aligned}
\mathrm{d} P(t) & =-\alpha_{\dagger} P \mathrm{~d} t+\left(\frac{2 \alpha_{\dagger} P^{0}}{\beta}\right)^{1 / 2} \bullet \mathrm{d} B(t) \\
& =-\alpha_{\dagger}\left(\frac{\beta P^{0}-1}{\beta P^{0}}\right) P \mathrm{~d} t+\left(\frac{2 \alpha_{\dagger} P^{0}}{\beta}\right)^{1 / 2} * \mathrm{~d} B(t) .
\end{aligned}
$$

The corresponding SDEs for the velocity process $V(t)=P / P^{0}$ are given by

$$
\begin{aligned}
\mathrm{d} V(t) & =-\alpha_{\dagger}\left[\left(1-V^{2}\right)-\frac{3}{\chi}\left(1-V^{2}\right)^{3 / 2}\right] V \mathrm{~d} t+\left[\frac{2 \alpha_{\dagger}}{\chi}\left(1-V^{2}\right)^{5 / 2}\right]^{1 / 2} \bullet \mathrm{d} B(t) \\
& =-\alpha_{\dagger}\left[\left(1-V^{2}\right)+\frac{2}{\chi}\left(1-V^{2}\right)^{3 / 2}\right] V \mathrm{~d} t+\left[\frac{2 \alpha_{\dagger}}{\chi}\left(1-V^{2}\right)^{5 / 2}\right]^{1 / 2} * \mathrm{~d} B(t) .
\end{aligned}
$$

With regard to numerical simulations, the Ito form is more convenient, cf. Appendix A.

Various properties of the RBM process (155) have been analyzed by Fa [24], Lindner [25], Fingerle, [395] ${ }^{68}$ and Angst and Franchi [15]. In particular, from Eq. (155e) we see that this model corresponds to

$$
\begin{aligned}
& \mu_{*}(v)=\alpha_{\dagger}\left[\left(1-v^{2}\right)+\frac{2}{\chi}\left(1-v^{2}\right)^{3 / 2}\right] v, \\
& b(v)=\frac{\alpha_{\dagger}}{\chi}\left(1-v^{2}\right)^{5 / 2},
\end{aligned}
$$

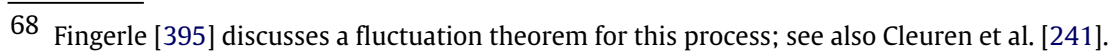




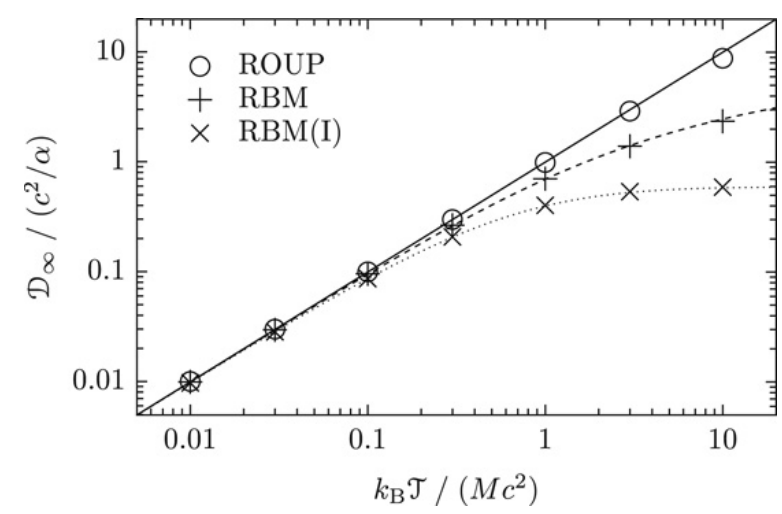

Fig. 7. Temperature dependence of the asymptotic diffusion constant $\mathscr{D}_{\infty}$ for the ROUP [11] from Eq. (151d), the RBM model [17] from Eq. (155b), and the RBM(I) model from Eq. (159a). Symbols 'o', ' + ' and ' $X$ ' represent the results of computer simulations $(N=100$ trajectories, initial conditions $X(0)=0$, $P(0)=0$ for each trajectory, discretization time step $\Delta t=10^{-4}$ in units of $\alpha_{c / \dagger / *}^{-1}$ ), obtained by averaging over the numerically determined values $\left\{\mathscr{D}_{100}, \mathscr{D}_{110}, \mathscr{D}_{120}, \ldots, \mathscr{D}_{500}\right\}$. Solid, dashed and dotted lines correspond to the theoretical predictions from Eqs. (154), (157) and (161), respectively.

with an upper velocity bound $v_{+}=c=1$ in Eq. (149). As for the ROUP, the integral formula (149) can be calculated analytically by making use of the identity (153) and one then finds that ${ }^{69}$

$$
D_{\infty}^{\mathrm{RBM}}=\left(\alpha_{\dagger} \chi\right)^{-1} \frac{K_{0}(\chi)}{K_{1}(\chi)}
$$

At low temperatures $\beta:=\left(k_{\mathrm{B}} \mathcal{T}\right)^{-1} \rightarrow \infty$, Eq. (157) reduces to the well-known classical result $\mathscr{D}_{\infty}^{\text {class }}=k_{\mathrm{B}} \mathcal{T} /\left(M \alpha_{\dagger}\right)$, cf. Eq. (33). In the opposite limit of very high temperatures (i.e., for $\beta M \ll 1$ ) one finds a logarithmic dependence [15]

$$
\mathcal{D}_{\infty}^{\mathrm{RBM}}=\left(\alpha_{\dagger} M\right)^{-1}\left\{-\gamma_{\epsilon}+\ln (2 / \chi)+\mathcal{O}\left[(\beta M)^{2}\right]\right\},
$$

where $\gamma_{\epsilon} \simeq 0.577216$ is the Euler constant. However, it should be kept in mind that, due to the increasing importance of particle annihilation/creation at high energies, classical non-quantum theories become invalid in the high temperature limit $\chi=\beta M \ll 1$, and, therefore, the asymptotic expansion (158) appears to be of limited practical use.

By comparing with the ROUP, we observe that $\mathscr{D}_{\infty}^{\mathrm{RBM}} \leq \mathcal{D}_{\infty}^{\mathrm{ROUP}}$ holds true for same values of the friction coefficients $\alpha_{c}=\alpha_{+}$, cf. Fig. 7. Intuitively, this can be explained by the fact that, for the ROUP, the absolute value of the friction force is bounded by $\alpha_{c} M$, cf. Eq. (151d), whereas the friction force is unbounded for the RBM model (155b), thereby suppressing spatial diffusion more strongly in the latter case.

Constant friction coefficient in the Ito SDE. The RBM process defined by Eq. (155b) is characterized by a constant friction coefficient $\alpha_{\dagger}$, when adopting the post-point discretization rule $(\bullet)$. Another model, referred to as RBM(I) hereafter, is obtained by considering a constant friction coefficient $\alpha_{*}$ in the Ito Langevin equation

$$
\mathrm{d} P(t)=-\alpha_{*} P \mathrm{~d} t+\left[\frac{2 \alpha_{*}}{\beta^{2}}\left(1+\beta P^{0}\right)\right]^{1 / 2} * \mathrm{~d} B(t),
$$

where the noise amplitude is chosen such that the Einstein relation (142c) is satisfied. The Ito SDE of the associated velocity process $V(t):=P / P^{0}$ reads

$$
\begin{aligned}
\mathrm{d} V(t)= & -\alpha_{*}\left[\left(1-V^{2}\right)+\frac{3}{\chi}\left(1-V^{2}\right)^{3 / 2}+\frac{3}{\chi^{2}}\left(1-V^{2}\right)^{2}\right] V \mathrm{~d} t \\
& +\left\{\frac{2 \alpha_{*}}{\chi}\left[\left(1-V^{2}\right)^{5 / 2}+\chi^{-1}\left(1-V^{2}\right)^{3}\right]\right\}^{1 / 2} * \mathrm{~d} B(t) .
\end{aligned}
$$

In this case, we have

$$
\begin{aligned}
& \mu_{*}(v)=\alpha_{*}\left[\left(1-v^{2}\right)+\frac{3}{\chi}\left(1-v^{2}\right)^{3 / 2}+\frac{3}{\chi^{2}}\left(1-v^{2}\right)^{2}\right] v, \\
& b(v)=\frac{\alpha_{*}}{\chi}\left[\left(1-v^{2}\right)^{5 / 2}+\chi^{-1}\left(1-v^{2}\right)^{3}\right] .
\end{aligned}
$$

From these equations the velocity potential is obtained as

$$
U(v)=\ln \left[\frac{\chi+1}{\chi\left(1-v^{2}\right)+\left(1-v^{2}\right)^{3 / 2}}\right]-\chi\left[1-\left(1-v^{2}\right)^{-1 / 2}\right]
$$

69 Eq. (157) is an equivalent, more compact representation of Lindner's result Eq. (10) in [25]. 
yielding for the asymptotic diffusion constant:

$$
\mathscr{D}_{\infty}^{\mathrm{RBM}(\mathrm{I})}=\left[\alpha_{*} K_{1}(\chi)\right]^{-1} \int_{0}^{1} \mathrm{~d} v \frac{\mathrm{e}^{-\chi\left(1-v^{2}\right)^{-1 / 2}}}{\chi\left(1-v^{2}\right)+\left(1-v^{2}\right)^{3 / 2}} .
$$

The remaining integral can be evaluated numerically. As illustrated in Fig. 7, the theoretical predictions from Eqs. (157), (154) and (161) are in good agreement with the numerically obtained estimates of the asymptotic diffusion constant.

The three model processes (151d), (155b) and (159a) give rise to the same stationary momentum PDF $\phi_{\mathrm{J}}(p)$, but their respective relaxation behavior differs strongly. This is illustrated in Fig. 6, which depicts the time evolution of the spatial mean square displacement divided by time,

$$
\mathcal{D}_{t}:=\left\langle[X(t)-X(0)]^{2}\right\rangle /(2 t),
$$

for all three models at same temperature $\mathcal{T}$. The curves in Fig. 6 were calculated numerically from Eqs. (151d), (155c) and (159a), respectively, using an algorithm similar to those described in [15,25], see also Appendix A.5.

The ROUP and the two RBM models considered in this part represent special limit cases of the general Langevin equation (141) with arbitrarily chosen friction coefficient functions. Nonetheless, they yield useful insights: As evident from Fig. 7, at moderate-to-high temperatures the diffusion constant can vary significantly for different friction models. For realistic systems, the exact functional shape (i.e., energy dependence) of the friction function $\alpha$ is determined by the microscopic interactions. This result implies that simultaneous measurements of the temperature and the diffusion constants can reveal information about the underlying microscopic forces. Below, in Section 4.5, we will outline a general procedure for deducing more realistic friction coefficients $\alpha$ from microscopic models.

\subsection{Proper-time reparameterization}

The relativistic Langevin equations considered thus far are parameterized in terms of the time-coordinate $t$ of the lab frame $\Sigma$. We shall now discuss how these equations can be re-parameterized in terms of the proper-time $\tau$. For this purpose, we start from the Ito Langevin equation ${ }^{70}$

$$
\begin{aligned}
& \mathrm{d} X^{\alpha}(t)=\left(P^{\alpha} / P^{0}\right) \mathrm{d} t, \\
& \mathrm{~d} P^{i}(t)=A^{i} \mathrm{~d} t+c^{i}{ }_{j} * \mathrm{~d} B^{j}(t) .
\end{aligned}
$$

In general, the functions $A^{i}$ and $C_{j}^{i}$ may depend on $\left(t, X^{i}, P^{i}\right)$. Upon applying Ito's formula [74,500] to the mass-shell condition $P^{0}(t)=\left(M^{2}+\boldsymbol{P}^{2}\right)^{1 / 2}$, Eq. (163b) yields the following equation for the relativistic energy

$$
\mathrm{d} P^{0}(t)=\left\{A_{i} \frac{P^{i}}{P^{0}}+\frac{D_{i j}}{2}\left[\frac{\delta^{i j}}{P^{0}}-\frac{P^{i} P^{j}}{\left(P^{0}\right)^{3}}\right]\right\} \mathrm{d} t+\frac{P^{i}}{P^{0}} c_{i r} * \mathrm{~d} B^{r}(t),
$$

where $A_{i}:=A^{i}, D_{i j}:=D^{i j}=c_{r}^{i} c_{r}^{j}:=\sum_{r} c_{r}^{i} c_{r}^{j}$ and $c_{i r}:=c_{r}^{i}$. The Fokker-Planck equation for the associated phase space PDF $f(t, \boldsymbol{x}, \boldsymbol{p})$ reads

$$
\left(\frac{\partial}{\partial t}+\frac{p^{i}}{p^{0}} \frac{\partial}{\partial x^{i}}\right) f=\frac{\partial}{\partial p^{i}}\left[-A^{i} f+\frac{1}{2} \frac{\partial}{\partial p^{k}}\left(D^{i k} f\right)\right] .
$$

As before, we consider deterministic initial data $\boldsymbol{X}(0)=\boldsymbol{x}_{0}$ and $\boldsymbol{P}(0)=\boldsymbol{p}_{0}$, which translates into $f(0, \boldsymbol{x}, \boldsymbol{p})=\delta\left(\boldsymbol{x}-\boldsymbol{x}_{0}\right)$ $\delta\left(\boldsymbol{p}-\boldsymbol{p}_{0}\right)$.

We are interested in rewriting Eq. (163) in terms of the proper-time $\tau$, defined by

$$
\mathrm{d} \tau(t)=\left(1-\boldsymbol{V}^{2}\right)^{1 / 2} \mathrm{~d} t, \quad \tau(0)=0 .
$$

The proper-time differential may also be expressed as

$$
\mathrm{d} \tau(t)=\left(M / P^{0}\right) \mathrm{d} t .
$$

The inverse of the function $\tau(t)$ will be denoted by $\hat{X}^{0}(\tau)=t(\tau)$ and represents the time coordinate of the particle in the frame $\Sigma$ parameterized by the proper time $\tau$. Our goal is to find SDEs for the reparameterized processes $\hat{X}^{\alpha}(\tau):=X^{\alpha}(t(\tau))$ and $\hat{P}^{\alpha}(\tau)=P^{\alpha}(t(\tau))$ in $\Sigma$. Heuristically, the SDEs can be derived from the relation

$$
\mathrm{d} B^{j}(t) \simeq \sqrt{\mathrm{d} t}=\left(\hat{P}^{0} / M\right)^{1 / 2} \sqrt{\mathrm{d} \tau} \simeq\left(\hat{P}^{0} / M\right)^{1 / 2} * \mathrm{~d} \hat{B}^{j}(\tau),
$$

where $\hat{B}^{j}(\tau)$ is a standard Wiener process with time-parameter $\tau$. Equation (166b) can be justified rigorously by applying theorems for the time-change of (local) martingale processes [30,500]. Inserting Eqs. (166) in Eqs. (132) one finds

\footnotetext{
70 For simplicity, we assume that $\boldsymbol{B}(t)=\left(B^{j}(t)\right)$ is $d$-dimensional, implying that $C_{j}^{i}$ is a square matrix. However, all results still hold if $\boldsymbol{B}(t)$ has a different dimension.
} 


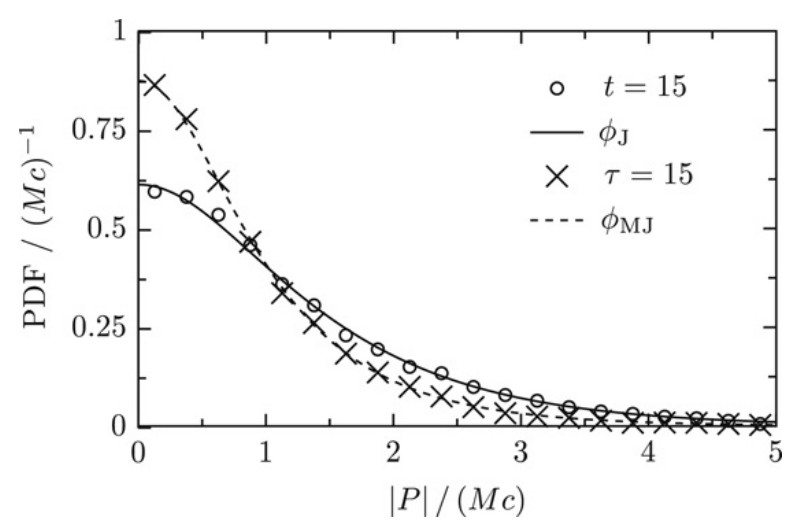

Fig. 8. 'Stationary' probability density function (PDF) of the absolute momentum $|P|$ measured at time $t=15(x)$ and proper-time $\tau=15(\bigcirc)$, respectively, from 10000 sample trajectories of the one-dimensional $(d=1)$ relativistic Ornstein-Uhlenbeck process [11], corresponding to coefficients $D\left(p^{0}\right)=D_{c}=$ const and $\alpha\left(p^{0}\right)=\beta D_{c} / p^{0}$ in Eqs. (163) and (169). Simulation parameters: $\mathrm{d} t=0.001, M=c=\beta=D_{c}=1$. The solid and dashed lines show the corresponding standard and modified Jüttner functions $\phi_{\mathrm{J}} \propto \exp \left(-\beta p^{0}\right)$ and $\phi_{\mathrm{MJ}} \propto \exp \left(-\beta p^{0}\right) / p^{0}$, respectively.

$$
\begin{aligned}
& \mathrm{d} \hat{X}^{\alpha}(\tau)=\left(\hat{P}^{\alpha} / M\right) \mathrm{d} \tau, \\
& \mathrm{d} \hat{P}^{i}(\tau)=\hat{A}^{i} \mathrm{~d} \tau+\hat{c}_{j}^{i} * \mathrm{~d} \hat{B}^{j}(\tau),
\end{aligned}
$$

where the transformed coefficients are given by

$$
\begin{aligned}
& \hat{A}^{i}:=\left(\hat{P}^{0} / M\right) A^{i}\left(\hat{X}^{0}, \hat{\boldsymbol{X}}, \hat{\boldsymbol{P}}\right), \\
& \hat{c}_{j}^{i}:=\left(\hat{P}^{0} / M\right)^{1 / 2} c_{j}^{i}\left(\hat{X}^{0}, \hat{\boldsymbol{X}}, \hat{\boldsymbol{P}}\right) .
\end{aligned}
$$

Analogous to Eq. (163c), the reparameterized energy equation is obtained as

$$
\mathrm{d} \hat{P}^{0}(\tau)=\left\{\hat{A}_{i} \frac{\hat{P}^{i}}{\hat{P}^{0}}+\frac{\hat{D}_{i j}}{2}\left[\frac{\delta^{i j}}{\hat{P}^{0}}-\frac{\hat{P}^{i} \hat{P}^{j}}{\left(\hat{P}^{0}\right)^{3}}\right]\right\} \mathrm{d} t+\frac{\hat{P}^{i}}{\hat{P}^{0}} \hat{c}_{i r} * \mathrm{~d} \hat{B}^{r}(\tau)
$$

where now $\hat{D}^{i k}:=\hat{c}_{r}^{i} \hat{c}_{r}^{k}$. The FPE for the associated probability density $\hat{f}\left(\tau, x^{0}, \boldsymbol{x}, \boldsymbol{p}\right)$ reads

$$
\left(\frac{\partial}{\partial \tau}+\frac{p^{\alpha}}{M} \frac{\partial}{\partial x^{\alpha}}\right) \hat{f}=\frac{\partial}{\partial p^{i}}\left[-\hat{A}^{i} \hat{f}+\frac{1}{2} \frac{\partial}{\partial p^{k}}\left(\hat{D}^{i k \hat{f}}\right)\right] .
$$

We note that now $\hat{f} \mathrm{~d} x^{0} \mathrm{~d}^{d} x \mathrm{~d}^{d} p$ gives the probability of finding the particle at proper-time $\tau$ in the $(2 d+1)$-dimensional interval $[t, t+\mathrm{d} t] \times[\boldsymbol{x}, \boldsymbol{x}+\mathrm{d} \boldsymbol{x}] \times[\boldsymbol{p}, \boldsymbol{p}+\mathrm{d} \boldsymbol{p}]$ in the inertial frame $\Sigma$.

An interesting consequence of this reparameterization can be illustrated by considering the free motion in a thermalized heat bath, which is stationary, isotropic and position independent in the lab frame $\Sigma$. As discussed above, in this case a plausible ansatz reads

$$
A^{i}=-\hat{\alpha}\left(p^{0}\right) p^{i}, \quad c_{j}^{i}=\left[2 \hat{D}\left(p^{0}\right)\right]^{1 / 2} \delta_{j}^{i},
$$

where the friction and noise coefficients $\hat{\alpha}$ and $\hat{D}$ depend on the energy $p^{0}=\left(M^{2}+\boldsymbol{p}^{2}\right)^{1 / 2}$ and satisfy the relativistic Einstein relation

$$
0 \equiv \hat{\alpha}\left(p^{0}\right) p^{0}+\hat{D}^{\prime}\left(p^{0}\right)-\beta D\left(p^{0}\right) .
$$

Then, the stationary momentum distribution of the lab-time FPE (164) is given by a Jüttner function [186,202], i.e.,

$$
f_{\infty}:=\lim _{t \rightarrow \infty} f \propto \frac{\exp \left(-\beta p^{0}\right)}{Z_{\mathrm{J}}}=\phi_{\mathrm{J}}(\boldsymbol{p}) .
$$

Remarkably, however, in this case the stationary solution $\hat{f}_{\infty}$ of the corresponding proper-time FPE (168) is given by the modified Jüttner function [475]

$$
\hat{f}_{\infty}:=\lim _{\tau \rightarrow \infty} \hat{f} \propto \frac{\exp \left(-\beta p^{0}\right)}{Z_{\mathrm{J}} p^{0}}=: \phi_{\mathrm{MJ}}(\boldsymbol{p})
$$

This can be confirmed by direct numerical simulation of Eqs. (163). An example is given in Fig. 8, which depicts the numerically obtained PDF of the absolute momentum $|P|$ for the one-dimensional ROUP [11]. The numerical PDFs were 
calculated from 10000 sample trajectories, by measuring the momentum at constant lab-time $t(x)$ and constant propertime $\tau(\circ)$, respectively. The solid and dashed lines show the corresponding standard and modified Jüttner functions, respectively. The physical explanation for the difference between $f_{\infty}$ and $\hat{f}_{\infty}$ is that measurements at $t=$ const and $\tau=$ const are non-equivalent even if both $\tau, t \rightarrow \infty$.

The modified Jüttner distribution $\phi_{\mathrm{M}]}$, representing the stationary solution of the proper-time FPE, differs from the standard Jüttner function $\phi_{\mathrm{J}}(118 \mathrm{~b})$ by a prefactor proportional to the inverse energy. As discussed in Ref. [475], the modified distribution $\phi_{\mathrm{MJ}}$ can be derived from a relative entropy principle by using a Lorentz invariant reference measure in momentum space, whereas the Jüttner function $\phi_{\mathrm{J}}$ is obtained when adopting a constant reference density (Lebesgue measure), cf. Eq. (123a).

We may summarize: If a relativistic Langevin-Ito process has been specified in the inertial frame $\Sigma$ and is parameterized by the associated $\Sigma$-coordinate time $t$, then this process can be reparameterized by its proper-time $\tau$ and the resulting process is again of the Langevin-Ito type but exhibits a modified stationary distribution. With regard to applications [316, 319] the latter fact means that the correct form of the fluctuation-dissipation relation depends on the choice of the timeparameter in the relativistic Langevin equation. In principle, however, one can - similar to the case of purely deterministic relativistic equations of motions - choose freely between different time parameterizations by taking into account that the noise part needs to be transformed differently than the deterministic part.

\subsection{Moving observers}

The preceding discussion has focused on Langevin equations that describe the random motion of a relativistic Brownian particle in the lab frame, defined as the rest frame of the heat bath. In this part we would like to address the following question [26,30]: Assuming that a Langevin equation of the type (141) holds in the lab frame $\Sigma-$ how does the corresponding process appear to a moving observer?

There exist (at least) two different ways to tackle this problem: Either one uses the Lorentz invariance of the phase space density, or one applies a Lorentz transformation directly to the Langevin equation $[26,30]$.

\subsubsection{Lorentz transformation of the phase space density}

An observer at rest in the lab frame would measure the PDF $f$ governed by the Fokker-Planck equations (135) or (138). As discussed in Section 3.1.2 the one-particle phase space density transforms as a Lorentz scalar [151]; i.e.,

$$
f^{\prime}\left(t^{\prime}, \boldsymbol{x}^{\prime}, \boldsymbol{p}^{\prime}\right)=f\left(t\left(t^{\prime}, \boldsymbol{x}^{\prime}\right), \boldsymbol{x}\left(t^{\prime}, \boldsymbol{x}^{\prime}\right), \boldsymbol{p}\left(\boldsymbol{p}^{\prime}\right)\right),
$$

and, conversely,

$$
f(t, \boldsymbol{x}, \boldsymbol{p})=f^{\prime}\left(t^{\prime}(t, \boldsymbol{x}), \boldsymbol{x}^{\prime}(t, \boldsymbol{x}), \boldsymbol{p}^{\prime}(\boldsymbol{p})\right),
$$

where $\left(t^{\prime}, \boldsymbol{x}^{\prime}, \boldsymbol{p}^{\prime}\right)$ and $(t, \boldsymbol{x}, \boldsymbol{p})$ are related by the Lorentz transformation

$$
\begin{aligned}
& x^{\prime \lambda}(t, \boldsymbol{x})=\Lambda^{\lambda}{ }_{0} t+\Lambda^{\lambda}{ }_{i} x^{i}, \\
& p^{\prime i}(\boldsymbol{p})=\Lambda_{0}^{i}\left(m^{2}+\boldsymbol{p}^{2}\right)^{1 / 2}+\Lambda_{j}^{i} p^{j} .
\end{aligned}
$$

For an observer moving at constant velocity $\boldsymbol{w}$ through the lab frame, the corresponding Lorentz boost matrix elements are given by Eq. (83). Therefore, in order to find $f^{\prime}$, it suffices to solve the Fokker-Planck equations (135) in the lab frame $\Sigma$, e.g., for a given $t$-simultaneous initial condition $f(0, \boldsymbol{x}, \boldsymbol{p})$, and to insert the solution into (171a).

\subsubsection{Lorentz transformation of the Langevin equation}

Alternatively, in order to obtain explicit SDEs for the Brownian motion in $\Sigma^{\prime}$, one has to apply a Lorentz transformation to the Langevin equations in the lab frame $\Sigma[26,30]$. This can be done by employing similar heuristics as in the case of the proper-time reparameterization, cf. Section 4.3 .

To illustrate this in more detail, we start again from the Langevin equations (163) and consider a Lorentz transformation [8] from the lab frame $\Sigma$ to another inertial frame $\Sigma^{\prime}$, mediated by a constant matrix $\Lambda^{v}{ }_{\mu}$ that leaves the metric tensor $\eta_{\alpha \beta}$ invariant. For convenience, we exclude time reversal transformations, i.e., we restrict ourselves to proper Lorentz transformations with $\Lambda^{0}{ }_{0}>0$. It is convenient to proceed in two steps: First we define

$$
Y^{\prime \nu}(t):=\Lambda^{\nu}{ }_{\mu} X^{\mu}(t), \quad G^{\prime \nu}(t):=\Lambda^{\nu}{ }_{\mu} P^{\mu}(t) .
$$

Then we replace $t$ by the coordinate time $t^{\prime}$ of $\Sigma^{\prime}$ to obtain processes

$$
X^{\prime \alpha}\left(t^{\prime}\right)=Y^{\prime \alpha}\left(t\left(t^{\prime}\right)\right), \quad P^{\prime \alpha}\left(t^{\prime}\right)=G^{\prime \alpha}\left(t\left(t^{\prime}\right)\right) .
$$

In order to find the SDEs for $X^{\prime \alpha}\left(t^{\prime}\right)$ and ${P^{\prime \alpha}}^{\alpha}\left(t^{\prime}\right)$, we note that $\mathrm{d}^{\prime 0}(t)=\Lambda^{0}{ }_{\mu} \mathrm{d} X^{\mu}(t)$ and, therefore,

$$
\mathrm{d} t^{\prime}(t)=\mathrm{d} Y^{\prime 0}(t)=\frac{\Lambda^{0}{ }_{\mu} P^{\mu}}{P^{0}} \mathrm{~d} t=\frac{G^{0}}{P^{0}} \mathrm{~d} t=\frac{P^{\prime 0}\left(t^{\prime}(t)\right)}{\left(\Lambda^{-1}\right)_{\mu}^{0} P^{\prime \mu}\left(t^{\prime}(t)\right)} \mathrm{d} t
$$


where $\Lambda^{-1}$ is the inverse Lorentz transformation. A similar heuristics as in Eq. (166b) then gives

$$
\mathrm{d} B^{j}(t) \simeq \sqrt{\mathrm{d} t}=\left(\frac{P^{0}}{P^{\prime 0}}\right)^{1 / 2} \sqrt{\mathrm{d} t^{\prime}} \simeq\left[\frac{\left(\Lambda^{-1}\right)_{\mu}^{0} P^{\prime \mu}}{P^{\prime 0}}\right]^{1 / 2} * \mathrm{~d} B^{j}\left(t^{\prime}\right),
$$

where $B^{j}\left(t^{\prime}\right)$ is a Wiener process with time parameter $t^{\prime}$. Defining

$$
\begin{aligned}
& A^{0}:=A_{i} \frac{p^{i}}{p^{0}}+\frac{D_{i j}}{2}\left[\frac{\delta^{i j}}{p^{0}}-\frac{p^{i} p^{j}}{\left(p^{0}\right)^{3}}\right], \\
& c_{j}^{0}:=\frac{p^{i}}{p^{0}} c_{i j},
\end{aligned}
$$

we can introduce Lorentz-transformed coefficient functions in $\Sigma^{\prime}$ by

$$
\begin{aligned}
& A^{\prime i}\left(x^{\prime 0}, \boldsymbol{x}^{\prime}, \boldsymbol{p}^{\prime}\right)=\left[\frac{\left(\Lambda^{-1}\right)^{0}{ }_{\mu} p^{\prime \mu}}{p^{0}}\right] \Lambda_{\nu}^{i} A^{v}\left(\left(\Lambda^{-1}\right)^{0}{ }_{\mu} x^{\prime \mu},\left(\Lambda^{-1}\right)^{i}{ }_{\mu} x^{\prime \mu},\left(\Lambda^{-1}\right)_{\mu}^{i} p^{\prime \mu}\right), \\
& c^{\prime i}{ }_{j}\left(x^{\prime 0}, \boldsymbol{x}^{\prime}, \boldsymbol{p}^{\prime}\right):=\left[\frac{\left(\Lambda^{-1}\right)^{0}{ }_{\mu} p^{\prime \mu}}{p^{\prime 0}}\right]^{1 / 2} \Lambda^{i}{ }_{\nu} c^{\nu}{ }_{j}\left(\left(\Lambda^{-1}\right)^{0}{ }_{\mu}{x^{\prime \mu}}^{\prime \mu},\left(\Lambda^{-1}\right)^{i}{ }_{\mu} x^{\prime \mu},\left(\Lambda^{-1}\right)^{i}{ }_{\mu} p^{\prime \mu}\right) .
\end{aligned}
$$

Substituting the formulas (173)-(175) into the Langevin equations (163), one finds that the particle's trajectory $\left(\boldsymbol{X}^{\prime}\left(t^{\prime}\right), \boldsymbol{P}^{\prime}\left(t^{\prime}\right)\right)$ in $\Sigma^{\prime}$ is governed by an SDE of the form $[11,17,20]$

$$
\begin{aligned}
& \mathrm{d} X^{\prime \alpha}\left(t^{\prime}\right)=\left({P^{\prime \alpha}}^{\prime \alpha} P^{\prime 0}\right) \mathrm{d} t^{\prime}, \\
& \mathrm{d} P^{\prime i}\left(t^{\prime}\right)=A^{\prime i} \mathrm{~d} t^{\prime}+{c^{\prime}}^{i}{ }_{j} * \mathrm{~d} B^{j}\left(t^{\prime}\right) .
\end{aligned}
$$

It should be noted that, due to the [ $\cdot]$-prefactors in Eq. (175), the coefficients $A^{\mu}$ and $c^{v}{ }_{\mu}$ do not form Lorentz tensors. This is essentially a consequence of the coordinate-time parameterization in Eqs. (163) and (176). ${ }^{71}$ Furthermore, it is straightforward now to obtain the corresponding SDE for the energy ${P^{\prime}}^{0}=\left(M^{2}+\boldsymbol{P}^{\prime 2}\right)^{1 / 2}$ in $\Sigma^{\prime}$ as well as the Fokker-Planck equation by replacing unprimed with primed quantities in Eqs. (163c) and (164), respectively. ${ }^{72}$

\subsection{Relativistic binary collision model}

The preceding subsections have focused on general aspects of relativistic Langevin and Fokker-Planck equations. Similar to the nonrelativistic case, relativistic SDEs present a useful tool for analytical and numerical studies of relaxation processes in relativistic systems. Stochastic models of this type provide a simplified picture of the underlying microscopic dynamics. In order for the Langevin approach to be successful, one must know in advance which friction coefficient function $\alpha(P)$ and noise amplitude $D(P)$ are appropriate for the system under consideration. Realistic friction models can be obtained, e.g., by deriving Fokker-Planck equations from relativistic Boltzmann equations $[16,299]$. In this context, however, it should be noted that the validity of the relativistic Boltzmann equation [312] is less understood than that of its nonrelativistic counterpart. In the remainder of this section, we will sketch an alternative procedure for obtaining friction coefficients and noise amplitudes from a simple microscopic interaction model [21]. The latter can be viewed as the direct relativistic generalization of the elastic binary collision model from Section 2.2.2. More precisely, we consider a one-dimensional system consisting of a heavy Brownian particle (mass $M$ ) which is embedded into a heat bath of smaller particles (mass $m \ll M$, total number $N \gg 1$ ). Our model assumes that the stochastic motion of a Brownian particle arises due to frequent elastic interactions with the surrounding heat bath particles. Similar to Section 2.2.2, we are interested in finding the 'best' approximation of the 'exact' dynamics within the class of SDEs defined by Eq. (141).

Relativistic collision kinematics. To begin with, we consider a single collision of the Brownian particle (momentum $P$, energy $E=P^{0}$ ) with a heat bath particle (momentum $p$, energy $\epsilon$ ). The relativistic energy, momentum and velocity of the two particles before the collision are given by

$$
\begin{aligned}
& P=M V \gamma(V), \quad E(P)=\left(M^{2}+P^{2}\right)^{1 / 2}, \\
& p=m v \gamma(v), \quad \epsilon(p)=\left(m^{2}+p^{2}\right)^{1 / 2} .
\end{aligned}
$$

\footnotetext{
71 The coefficients $\hat{A}^{i}$ of the corresponding $\tau$-parameterized process are the spatial components of a Lorentz four-vector $\hat{A}^{\mu}$; similarly, the quantities $\hat{D}^{i j}=\sum_{r} \hat{c}_{r}^{i} \hat{c}_{r}^{k}$ are spatial components of a second rank Lorentz tensor.

72 In particular, if the coefficients of the lab frame Langevin equation satisfy the relativistic Einstein relation (169b), then the stationary momentum distribution in the moving frame $\Sigma^{\prime}$ is given by a boosted Jüttner distribution of the form $\phi^{\prime}\left(\boldsymbol{p}^{\prime}\right) \propto \exp \left(\beta U_{\alpha}^{\prime} p^{\prime \alpha}\right)$, where $U_{\alpha}^{\prime}$ is the four-velocity of the heat bath in $\Sigma^{\prime}$.
} 
where $\gamma(v):=\left(1-v^{2}\right)^{-1 / 2}$. Considering elastic interactions, the collision kinematics is governed by the relativistic mass-energy-momentum conservation laws

$$
\hat{M}=M, \quad \hat{m}=m, \quad E+\epsilon=\hat{E}+\hat{\epsilon}, \quad P+p=\hat{P}+\hat{p},
$$

where hat-symbols refer to the state after the collision. Inserting Eqs. (177) into the conservation laws (178), and solving for the momentum of Brownian particle after the collision, $\hat{P}$, we obtain [21]

$$
\hat{P}=\gamma(u)^{2}\left[2 u E-\left(1+u^{2}\right) P\right],
$$

where the collision-invariant center-of-mass velocity $u$ is given by

$$
u(p, P)=\frac{P+p}{E+\epsilon} .
$$

Accordingly, the momentum change $\Delta P_{r}:=\hat{P}-P$ of the Brownian particle in a single collision with the heat bath particle ' $r$ ' is given by

$$
\Delta P_{r}=-2 \gamma\left(u_{r}\right)^{2} \frac{\epsilon_{r}}{E+\epsilon_{r}} P+2 \gamma\left(u_{r}\right)^{2} \frac{E}{E+\epsilon_{r}} p_{r},
$$

where $u_{r}:=u\left(p_{r}, P\right)$ and $\epsilon_{r}:=\epsilon\left(p_{r}\right)$. In the non-relativistic limit case, where $u_{r}^{2} \ll 1, E \simeq M$ and $\epsilon_{r} \simeq m$, Eq. (180) reduces to Eq. (48).

Furthermore, by making the same assumptions as in Section 2.2.2, we find that the momentum change $\delta P(t):=$ $P(t+\delta t)-P(t)$ of the Brownian particle during a small-but-sufficiently-long time interval $[t, t+\delta t]$ can be approximated by

$$
\delta P(t) \approx-2 \sum_{r=1}^{N} \gamma\left(u_{r}\right)^{2} \frac{\epsilon_{r}}{E+\epsilon_{r}} P(t) I_{r}(t, \delta t)+2 \sum_{r=1}^{N} \gamma\left(u_{r}\right)^{2} \frac{E}{E+\epsilon_{r}} p_{r} I_{r}(t, \delta t) .
$$

Formally, the collision indicator $I_{r}(t, \delta t)$ is again given by [cf. Eq. (53a)]

$$
I_{r}(t, \delta t) \approx \frac{\delta t}{2}\left|v_{r}-V\right| \delta\left(x_{r}-X\right),
$$

but now one has to use the relativistic velocities $V=P /\left(M^{2}+P^{2}\right)^{1 / 2}$ and $v_{r}=p_{r} /\left(M^{2}+p_{r}^{2}\right)^{1 / 2}$, respectively. Eq. (181a) is the relativistic counterpart of Eq. (53b). Heuristically, the first term on the rhs of Eq. (181a) can again be interpreted as 'friction', while the second contribution may be viewed as 'noise'.

Bath distribution and drift. Similar to the nonrelativistic case, Eqs. (181) can be used to calculate the statistical moments of the momentum increments - provided one specifies the phase space distribution of the heat bath particles. We will assume here that the heat bath is in a thermal equilibrium state, so that the one-particle phase space PDF is given by a spatially homogeneous Jüttner function on $\mathbb{V}=[0, L]$, i.e.,

$$
f_{\mathrm{b}}^{1}\left(x_{r}, p_{r}\right)=\left(\mathcal{Z}_{1} L\right)^{-1} \exp \left[-\beta\left(m^{2}+p_{r}^{2}\right)^{1 / 2}\right] \Theta\left(L-x_{r}\right) \Theta\left(x_{r}\right),
$$

where $\mathcal{T}=\left(\beta k_{\mathrm{B}}\right)^{-1}$ is the temperature, and $\mathcal{Z}_{1}=2 m K_{1}(\beta m)$ with $K_{1}(z)$ denoting the modified Bessel function. With regard to our subsequent discussion, we are interested in calculating the mean drift force $\mathcal{K}$, defined by ${ }^{73}$

$$
\mathcal{K}(p):=\left\langle\frac{\delta P(t)}{\delta t} \mid P(t)=p\right\rangle_{\mathrm{b}} .
$$

Inserting $\delta P(t)$ from Eq. (181a), we find

$$
\begin{aligned}
\mathcal{K}(p)= & -n_{\mathrm{b}}\left\langle\gamma\left(u_{r}\right)^{2} \frac{\epsilon_{r}}{E+\epsilon_{r}} L\left|v_{r}-V\right| \delta\left(x_{r}-X\right) \mid P(t)=p\right\rangle_{\mathrm{b}} P \\
& +n_{\mathrm{b}}\left\langle\gamma\left(u_{r}\right)^{2} \frac{E}{E+\epsilon_{r}} p_{r} L\left|v_{r}-V\right| \delta\left(x_{r}-X\right) \mid P(t)=p\right\rangle_{\mathrm{b}},
\end{aligned}
$$

where $n_{\mathrm{b}}=N / L$ is the number density of the bath particles. In order to determine $\mathcal{K}(p)$, we note that for some arbitrary function $G(p, P)$, we have

$$
\begin{aligned}
\left\langle G\left(p_{r}, P\right) L\left|v_{r}-V\right| \delta\left(x_{r}-X\right) \mid P(t)=p\right\rangle_{\mathrm{b}}= & Z_{1}^{-1} \int \mathrm{d} p_{r} G\left(p_{r}, P\right) \exp \left[-\beta\left(m^{2}+p_{r}^{2}\right)^{1 / 2}\right] \\
& \times\left|\frac{p_{r}}{\left(m^{2}+p_{r}^{2}\right)^{1 / 2}}-\frac{P}{\left(M^{2}+P^{2}\right)^{1 / 2}}\right| .
\end{aligned}
$$

\footnotetext{
73 In principle, higher moments can be calculated in a similar manner, but then one has to specify the corresponding many-particle heat bath PDFs.
} 


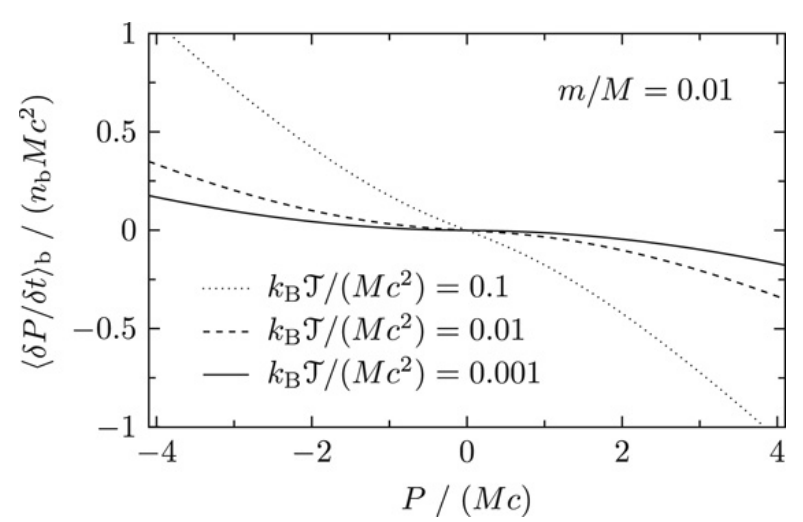

Fig. 9. Relativistic binary collision model. Mean drift force $\mathcal{K}(P):=\langle\delta P(t) / \delta t\rangle_{\mathrm{b}}$ numerically evaluated from Eq. (184) for different values $k_{\mathrm{B}} \mathcal{T}$, with $n_{\mathrm{b}}=N / L$ denoting the number density of the heat bath particles.

The rhs of Eq. (184) involves the functions

$$
\begin{aligned}
& G_{1}\left(p_{r}, P\right):=\gamma\left(u_{r}\right)^{2} \frac{\epsilon\left(p_{r}\right)}{E(P)+\epsilon\left(p_{r}\right)}, \\
& G_{2}\left(p_{r}, P\right):=\gamma\left(u_{r}\right)^{2} \frac{E(P)}{E(P)+\epsilon\left(p_{r}\right)} p_{r} .
\end{aligned}
$$

Unfortunately, it is very difficult or perhaps even impossible to analytically evaluate the integral (185) for the functions $G_{1 / 2}$. Fig. 9 depicts the mean drift force $\mathcal{K}$, obtained by numerically integrating the formula (185) for different values of $P$.

\section{Langevin approximation}

We conclude this section by discussing how one could, in principle, approximate Eqs. (181) by, for example, a nonlinear backward Ito SDE of the form (141), reading

$$
\mathrm{d} P(t)=-\alpha(P) P \mathrm{~d} t+[2 D(P)]^{1 / 2} \bullet \mathrm{d} B(t) .
$$

The considerations for the two-component Jüttner gas from Section 3 imply that the stationary momentum distribution of the Brownian particle in the binary collision model is given by the Jüttner function

$$
\phi_{\mathrm{J}}(p)=\exp \left[-\beta\left(p^{2}+M^{2}\right)^{1 / 2}\right] /\left[2 M K_{1}(\beta M)\right] .
$$

Hence, in order for Eq. (187) to yield the expected Jüttner distribution, the functions $\alpha$ and $D$ must be coupled by the relativistic Einstein relation (140),

$$
D(p)=\beta^{-1} \alpha(p) E(p),
$$

where $E=\left(p^{2}+M^{2}\right)^{1 / 2}$. In order to determine the function $\alpha$, we demand that the Langevin equation yields the same mean drift force $\mathcal{K}$ as the collision model, i.e.,

$$
\left\langle\frac{\mathrm{d} P(t)}{\mathrm{d} t} \mid P(t)=p\right\rangle \stackrel{!}{=}\left\langle\frac{\delta P(t)}{\delta t} \mid P(t)=p\right\rangle_{\mathrm{b}} .
$$

For the post-point (backward-Ito) Langevin equation (187) we know that [cf. Eq. (A.25)]

$$
\left\langle\frac{\mathrm{d} P(t)}{\mathrm{d} t} \mid P(t)=p\right\rangle=-\alpha(p) p+\frac{\mathrm{d}}{\mathrm{d} p} D(p) .
$$

Thus, by means of the Einstein relation (189), the lhs. of Eq. (190) is given by

$$
\left\langle\frac{\mathrm{d} P(t)}{\mathrm{d} t} \mid P(t)=p\right\rangle=-\alpha(p) p+\beta^{-1} \frac{\mathrm{d}}{\mathrm{d} p}[\alpha(p) E(p)],
$$

and the condition (190) becomes equivalent to the differential equation [cf. Eq. (65)]

$$
-\alpha(p) p+\beta^{-1} \frac{\mathrm{d}}{\mathrm{d} p}[\alpha(p) E(p)]=\mathcal{K}(p) .
$$

In the case of the collision model, where the function $\mathcal{K}(p)$ is not exactly known, one could, for example, try to fit $\mathcal{K}(p)$ by a simple analytic expression and, subsequently, use this approximation in Eq. (193). 


\section{Non-Markovian diffusion processes in Minkowski spacetime}

The preceding section has focused on relativistic Brownian motions in phase space. In the remainder we will discuss relativistic diffusion models in Minkowski spacetime, i.e., continuous relativistic stochastic processes that do not explicitly depend on the momentum coordinate. On the one hand, such spacetime processes may be constructed, for example, from a Brownian motion process in phase space by integrating out the momentum coordinates. As a result of this averaging procedure, the reduced process for the position coordinate will be non-Markovian. Alternatively, one can try to derive or postulate a relativistic diffusion equation and/or diffusion propagators in spacetime on the basis of microscopic models [10, $28,153,366$ ] or plausibility considerations [23]. Regardless of the approach adopted, in order to comply with the principles of special relativity, the resulting spacetime process must be non-Markovian, in accordance with the results of Dudley (Theorem 11.3 in [333]) and Hakim (Proposition 2 in [338]). Roughly speaking, this means that any relativistically acceptable generalization of the classical diffusion equation (1) should be of at least second order in the time coordinate.

The construction and analysis of relativistic diffusion models in Minkowski spacetime poses an interesting problem in its own right. Additionally, the investigation of these processes becomes relevant in view of potential analogies with relativistic quantum theory [383,391], similar to the analogy between Schrödinger's equation and the diffusion equation (1) in the nonrelativistic case [501,502]. The present section intends to provide an overview over classical relativistic diffusion models that have been discussed in the literature [10,23,27-29,337,366,367,379-381,391]. For this purpose, we first recall basic properties of the Wiener (Gaussian) process, which constitutes the standard paradigm for nonrelativistic diffusions in position space (Section 5.1). Subsequently, relativistic generalizations of the nonrelativistic diffusion equation (1) and/or the nonrelativistic Gaussian diffusion propagator will be discussed [23].

\subsection{Reminder: Nonrelativistic diffusion equation}

We start by briefly summarizing a few relevant facts about the standard nonrelativistic diffusion equation $[287,339,422$, 502]

$$
\frac{\partial}{\partial t} \varrho=D \nabla^{2} \varrho, \quad t \geq t_{0},
$$

where $\mathscr{D}>0$ denotes the spatial diffusion constant, and $\varrho(t, \boldsymbol{x}) \geq 0$ the one-particle PDF for the particle positions $\boldsymbol{x} \in \mathbb{R}^{d}$ at time $t$. Within classical diffusion theory, Eq. (194) is postulated to describe the (overdamped) random motion of a representative particle in a fluctuating environment (heat bath). In particular, Eq. (194) refers to the rest frame of the bath.

There exist several well-known ways to motivate or derive the phenomenological diffusion equation (194) by means of microscopic models (see, e.g., [287,339,422,502]). With regard to our subsequent discussion of relativistic alternatives, it is useful to briefly consider a 'hydrodynamic' derivation [503], which starts from the continuity equation

$$
\frac{\partial}{\partial t} \varrho(t, \boldsymbol{x})=-\nabla \cdot \boldsymbol{j}(t, \boldsymbol{x}),
$$

where $\boldsymbol{j}(t, \boldsymbol{x})$ denotes the current density vector. In order obtain a closed equation for the density $\varrho$, the current $\boldsymbol{j}$ has to be expressed in terms of $\varrho$. One way of doing this is to postulate the following rather general ansatz \{cf. Eq. (2.81) in [503]\}

$$
\boldsymbol{j}(t, \boldsymbol{x})=-\nabla \int_{t_{0}}^{t} \mathrm{~d} t^{\prime} K\left(t-t^{\prime}\right) \varrho\left(t^{\prime}, \boldsymbol{x}\right),
$$

where, in general, $K$ may be a memory kernel. However, considering for the moment the memory-less kernel function ${ }^{74}$

$$
K_{\mathrm{F}}\left(t-t^{\prime}\right):=2 \mathcal{D} \delta\left(t-t^{\prime}\right),
$$

one finds

$$
\boldsymbol{j}_{\mathrm{F}}(t, \boldsymbol{x})=-\mathscr{D} \nabla \varrho(t, \boldsymbol{x}) .
$$

Upon inserting this expression into the continuity equation (195), we recover the classical diffusion equation (194).

Now, it has been well-known for a long time that the diffusion equation (194) is in conflict with the postulates of special relativity. To briefly illustrate this, we specialize to simplest case of $d=1$ space dimensions, where $\nabla^{2}=\partial^{2} / \partial x^{2}$. In this case, the propagator of Eq. (194) at times $t>t_{0}$ is given by the Gaussian

$$
p\left(t, x \mid t_{0}, x_{0}\right)=\left[\frac{1}{4 \pi \mathscr{D}\left(t-t_{0}\right)}\right]^{1 / 2} \exp \left[-\frac{\left(x-x_{0}\right)^{2}}{4 \mathscr{D}\left(t-t_{0}\right)}\right] .
$$

The propagator (199) represents the solution of Eq. (194) for the initial condition

$$
\varrho\left(t_{0}, x\right)=\delta\left(x-x_{0}\right) .
$$

That is, if $X(t)$ denotes the random path of a particle with fixed initial position $X\left(t_{0}\right)=x_{0}$, then $p\left(t, x \mid t_{0}, x_{0}\right) \mathrm{d} x$ gives

$\overline{74}$ The factor '2' in Eq. (197) appears because of the convention $\int_{t_{0}}^{t} \mathrm{~d} t^{\prime} \delta\left(t-t^{\prime}\right) f\left(t^{\prime}\right)=f(t) / 2$. 


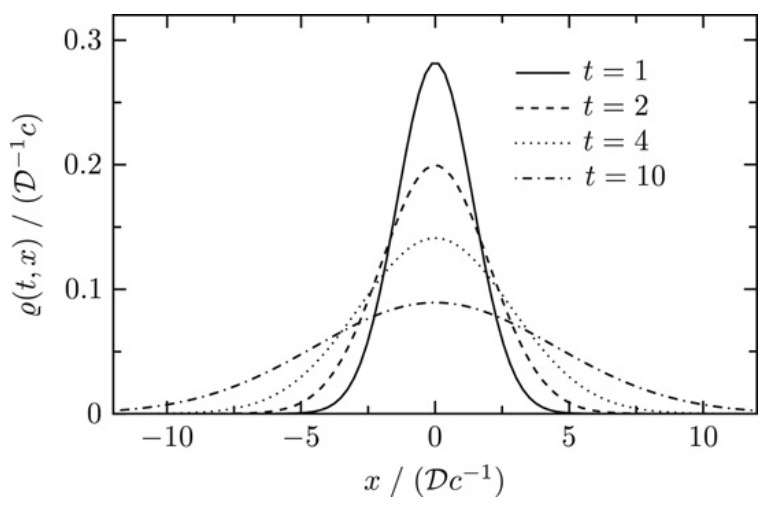

Fig. 10. Spreading of the Gaussian PDF $\varrho(t, x)=p(t, x \mid 0,0)$ from Eq. (199) at different times $t$, where $t$ is measured in units of $\mathscr{D} / c^{2}$. At initial time $t=t_{0}=0$, the PDF corresponds to a $\delta$-function centered at the origin.

the probability that the particle is found in the infinitesimal volume element $[x, x+\mathrm{d} x]$ at time $t>t_{0}$. As evident from Eq. (199), for each $t>t_{0}$ there is a small, but non-vanishing probability that the particle may be observed at distances $\left|x-x_{0}\right|>c\left(t-t_{0}\right)$, where $c=1$ is the speed of light in natural units. The evolution of the nonrelativistic Gaussian PDF from Eq. (199) is depicted in Fig. 10.

It is worthwhile to summarize a few essential properties of Eqs. (194) and (199): Eq. (194) is a linear parabolic partial differential equation. Due to the linearity, more general solutions may be constructed by superpositioning, i.e., by integrating the solution (199) over some given initial PDF $\varrho_{0}\left(x_{0}\right)$. Eq. (194) describes a Markov process which means that the transition PDF (199) satisfies the Chapman-Kolmogoroff criterion

$$
p\left(t, x \mid t_{0}, x_{0}\right)=\int_{\mathbb{R}} \mathrm{d} x_{1} p\left(t, x \mid t_{1}, x_{1}\right) p\left(t_{1}, x_{1} \mid t_{0}, x_{0}\right)
$$

for all $t_{1} \in\left(t_{0}, t\right)$. The corresponding diffusion process $X(t)$ can be characterized in terms of the following SDE:

$$
\mathrm{d} X(t)=(2 D))^{1 / 2} * \mathrm{~d} B(t), \quad X\left(t_{0}\right)=x_{0},
$$

where $B(t)$ is a standard Wiener process as defined in Section 2.1.2. Formally, Eq. (201) may be obtained from the Langevin equations (22) of the classical Ornstein-Uhlenbeck process with $\mathcal{F} \equiv 0$ as follows: First we rewrite Eq. (22b) as

$$
\frac{\mathrm{d} V(t)}{\alpha}=-V \mathrm{~d} t+\left(\frac{2 D_{0}}{M^{2} \alpha^{2}}\right)^{1 / 2} * \mathrm{~d} B(t) .
$$

Upon letting $\alpha \rightarrow \infty$ and $D_{0} \rightarrow \infty$ such that $\mathscr{D}=D_{0} /(\alpha M)^{2}$ remains constant, the lhs. of Eq. (202) should become negligible. Then, by making use of $\mathrm{d} X=V \mathrm{~d} t$, Eq. (201) is recovered. ${ }^{75}$ This limiting procedure defines the so-called overdamped regime of the Ornstein-Uhlenbeck process. The mean square displacement of the overdamped process (201) is given by [287]

$$
\begin{aligned}
\left\langle\left[X(t)-X\left(t_{0}\right)\right]^{2}\right\rangle & :=\int \mathrm{d} x\left(x-x_{0}\right)^{2} p\left(t, x \mid t_{0}, x_{0}\right) \\
& =2 \mathscr{D}\left(t-t_{0}\right),
\end{aligned}
$$

qualitatively similar to the asymptotic behavior of the classical Ornstein-Uhlenbeck process; cf. Eq. (25). Finally, we note that the solution of Eq. (194) with initial condition

$$
\varrho\left(t_{0}, x\right) \equiv \varrho_{0}(x),
$$

can be expressed in terms of the Feynman-Kac formula $[74,76]$

$$
\left.\varrho(t, x)=\left\langle\varrho_{0}(x+(2 D))^{1 / 2} B(t)\right)\right\rangle,
$$

where $\langle\cdot\rangle$ indicates an average with respect to the Wiener measure of the standard Wiener process $B(t)$ with initial condition $B\left(t_{0}\right)=0$. Equation (205) yields an efficient Monte-Carlo simulation scheme for computing the solutions of the diffusion equation (194) for a broad class of initial distributions $\varrho_{0}$.

\subsection{Telegraph equation}

The problem of constructing continuous diffusion models which, in contrast to the classical nonrelativistic equations (194) and (199), avoid superluminal velocities, has attracted considerable interest over the past years [10,23,27-29,327,337, 366,367,379-381,391]. Nonetheless, it seems fair to say that a commonly accepted solution is still outstanding. Apart from

\footnotetext{
75 Debbasch and Rivet [12] discuss the difficulties that arise when attempting a similar reduction for the relativistic Ornstein-Uhlenbeck process.
} 
the theoretical challenge of developing a consistent relativistic diffusion theory, there exist several practical applications including, for example, the analysis of data from high energy collision experiments [320-322] or the diffusion of light through turbid media [372,504,505] and foams [373-375]. In this context, a frequently considered alternative to the classical diffusion equation (194) is given by the telegraph equation $[10,27,29,320,321,366,370,377-380]$

$$
\left(\tau_{v} \frac{\partial^{2}}{\partial t^{2}}+\frac{\partial}{\partial t}\right) \varrho=\mathscr{D} \nabla^{2} \varrho .
$$

Here, $\mathscr{D}>0$ plays again the role of a diffusion constant, while $\tau_{v}>0$ is an additional relaxation time parameter. Equation (206) can also be obtained from the continuity equation (195), provided one uses the exponential memory kernel (Section 2.10 in Ref. [503])

$$
K_{\mathrm{T}}\left(t-t^{\prime}\right):=\frac{\mathscr{D}}{\tau_{v}} \exp \left[-\left(t-t^{\prime}\right) / \tau_{v}\right],
$$

instead of $K_{\mathrm{F}}$ from Eq. (197). Similar to Eq. (194), the telegraph equation (206) refers to a special frame where the background medium, causing the random motion of the diffusing test particle, is at rest (on average). The 'nonrelativistic limit' corresponds to letting $\tau_{v} \rightarrow 0$ in Eq. (206), which leads back to Eq. (194). For $\tau_{v}>0$, Eq. (206) is a linear hyperbolic partial differential equation. Because of the second order time derivative in Eq. (206), one now also has to specify the first order time derivative of the initial distribution at time $t_{0}$.

Considering again $d=1$ space dimensions and particular initial conditions

$$
\varrho\left(t_{0}, x\right)=\delta\left(x-x_{0}\right), \quad \frac{\partial}{\partial t} \varrho\left(t_{0}, x\right) \equiv 0,
$$

one finds that the corresponding solution of Eq. (206) is given by [10,366]

$$
p\left(t, x \mid t_{0}, x_{0}\right)=\frac{\mathrm{e}^{-\left(t-t_{0}\right) /\left(2 \tau_{v}\right)}}{2}\left\{\delta\left[\left|x-x_{0}\right|-v\left(t-t_{0}\right)\right]+\frac{\Theta\left(\xi^{2}\right)}{2 \tau_{v} v}\left[I_{0}(\xi)+\frac{t}{2 \tau_{v}} \frac{I_{1}(\xi)}{\xi}\right]\right\} .
$$

Here, we have abbreviated

$$
\xi:=\frac{1}{2}\left[\left(\frac{t-t_{0}}{\tau_{v}}\right)^{2}-\left(\frac{x-x_{0}}{\tau_{v} v}\right)^{2}\right]^{1 / 2}, \quad v:=\left(\mathscr{D} / \tau_{v}\right)^{1 / 2},
$$

and the modified Bessel functions of the first kind, $I_{v}(z)$, are defined by

$$
I_{v}(z):=\sum_{k=0}^{\infty} \frac{1}{\Gamma(k+v+1) k !}\left(\frac{z}{2}\right)^{2 k+v}
$$

with $\Gamma(z)$ denoting the Euler gamma function. According to our knowledge, the solution (209) was first obtained by Goldstein in 1938/1939. Actually, Goldstein derived the result (209) by considering the continuum limit of a persistent random walk model [369]; subsequently, he proved that this function satisfies the telegraph equation (206), cf. Section 8 of his paper [366].

The propagator (209) is characterized by two salient features:

(1) As evident from the $\delta$-function term, the solution exhibits two singular diffusion fronts traveling at absolute velocity $v:=\left(D / \tau_{v}\right)^{1 / 2}$ to the left and right, respectively.

(2) Due to the appearance of the Heaviside $\Theta$-function, the solution is non-zero only within the region $\left|x-x_{0}\right| \leq v\left(t-t_{0}\right)$, i.e., upon fixing $\tau_{v}$ such that $v=c=1$ the solution vanishes outside the light cone.

Thus, in contrast to the nonrelativistic propagator (199), Eqs. (209) define a relativistically acceptable diffusion model. Because of the second order time derivative, the telegraph equation (206) describes a non-Markovian process, in accordance with the aforementioned theorems of Dudley [333] and Hakim [338]. The non-Markovian character of the propagator (209) can also be proven directly by verifying that this solution does not fulfill the condition (200).

The linearity of Eq. (206) implies that more general solutions can be obtained by integrating the propagator (209) over some given initial distribution $\varrho_{0}\left(x_{0}\right)$. In principle, one may also construct other classes of solutions with $\partial \varrho\left(t_{0}, x\right) / \partial t \not \equiv 0$, e.g., by applying a Laplace-Fourier transformation [10,506,507] to Eq. (206). We note, however, that in order for the solution $\varrho(t, x)$ to remain normalized and positive at all times $t>t_{0}$, additional constraints on the initial conditions must be imposed, cf. Eq. (208). Various solutions and extensions of the telegraph equation (206), including different types of boundary conditions, additional external sources etc., have been discussed, e.g., by Goldstein [366], Masoliver et al. [506, 507], Foong [508], Foong and Kanno [509], Renardy [510], and Dorogovtsev [511].

Similar to the nonrelativistic diffusion equation (194), the telegraph equation (206) may be derived and/or motivated in many different ways. A detailed overview is given by Masoliver and Weiss [10], who discuss four different possibilities of deducing Eq. (206) from underlying models; see also Koide [27,29]. During the past decades, the telegraph equation (206) 
has been used to describe a number of different phenomena. The applications include:

- Transmission of electrical signals. According to Masoliver and Weiss (206), the earliest derivation of the telegraph equation is based on a paper by William Thomson [365] (who later became Lord Kelvin), published in 1855. He considered the problem of how to transmit electrical signals without distortion, a question closely related to the design of the first transatlantic cable.

- Continuum models of persistent diffusion. It seems that the concept of persistent diffusion was first proposed in 1917/1922 by Fürth $[368,369]$ who aimed at describing the random motion of biological objects. Independently, a similar approach was suggested by Taylor [370] in an attempt to treat turbulent diffusion [367]. Fürth and Taylor considered discrete models, assuming that a particle moves with constant absolute velocity between neighboring lattice points. At each lattice point, the particle is either back-scattered or transmitted, with the transmission probability being larger than the back-scattering probability (persistence). A few decades later, in 1950, Goldstein [366] demonstrated for the 1D case ${ }^{76}$ that, for a suitable choice of the transition probabilities, the continuum limit of this model leads to the telegraph equation. Hence, in contrast to the ordinary diffusion equation (194), the telegraph equation (206) relies on asymmetric transition probabilities, causing the non-vanishing probability concentration at the diffusion fronts. In more recent years, persistent diffusion models have been employed to describe the propagation of photons in thin slabs and foams [371-375].

- Heat transport and propagation of heat waves. In this case, the function $\varrho(t, x)$ in Eq. (206) is interpreted as a temperature field and the normalization condition is dropped. For a detailed account of the vast literature on heat waves, we refer to the review articles of Joseph and Preziosi [377,378].

- High energy ion collision experiments. In recent years, the telegraph equation has been used to estimate the dissipation of net charge fluctuations, which may obliterate signals of QCD phase transitions in nuclear collisions [320,321]. In this context, however, the coordinate $x$ in Eq. (206) is interpreted as a rapidity variable.

Another interesting aspect of the telegraph equation is elucidated in a paper by Kac [367]. He observed that the solutions of Eq. (206) with initial conditions

$$
\varrho\left(t_{0}, x\right) \equiv \varrho_{0}(x), \quad \frac{\partial}{\partial t} \varrho\left(t_{0}, x\right) \equiv 0,
$$

may be expressed in the form ${ }^{77}$

$$
\varrho(t, x)=\frac{1}{2}\left\langle\varrho_{0}\left(x-v \int_{t_{0}}^{t} \mathrm{~d} s(-1)^{N(s)}\right)\right\rangle+\frac{1}{2}\left\langle\varrho_{0}\left(x+v \int_{t_{0}}^{t} \mathrm{~d} s(-1)^{N(s)}\right)\right\rangle,
$$

where $v=\left(\mathscr{D} / \tau_{v}\right)^{1 / 2}$, and $\langle\cdot\rangle$ indicates an average with respect to the $\tau_{v}$-parameterized Poisson process $N(t)$; i.e., for any given time $t>t_{0}$ we have

$$
\operatorname{Prob}\{N(t)=k\}=\frac{\mathrm{e}^{-\left(t-t_{0}\right) /\left(2 \tau_{v}\right)}}{k !}\left(\frac{t-t_{0}}{2 \tau_{v}}\right)^{k}, \quad k=0,1,2, \ldots ;
$$

and for any finite sequence $t_{0}<t_{1}<\cdots<t_{n}$ the increments

$$
N\left(t_{1}\right)-N\left(t_{0}\right), N\left(t_{2}\right)-N\left(t_{1}\right), \ldots, N\left(t_{n}\right)-N\left(t_{n-1}\right)
$$

are independent. Equation (211) is the direct counterpart of Feynman-Kac formula (205) for the classical diffusion equation. Eqs. (211) and (212) together provide an efficient Monte-Carlo simulation scheme for computing solutions of the telegraph equation (206). Moreover, the Poisson path integral representation (211) discloses an interesting correspondence between the free-particle Dirac equation [382] and the telegraph equation (206), which was first pointed out by Gaveau et al. [383] in 1984: The solutions of both equations may be linked by means of an analytic continuation. This connection is similar to the relation between the diffusion equation (194) and the free particle Schrödinger equation in the nonrelativistic case. ${ }^{78}$ The crucial difference is given by the fact that the measures of the functional integration refer to different underlying processes, respectively.

However, the telegraph equation (206) is not the only possible relativistic generalization of the nonrelativistic diffusion equation (1) and, recently, there has been some controversy about its applicability and validity [379-381]. An early critical discussion of Eq. (206) in the context of relativistic heat transport was given by van Kampen [153] in 1970. Starting from a simple microscopic model, consisting of a cloud of material particles that exchange electromagnetic radiation, van Kampen derived an integral equation for the temperature of the particles as function of time and space. He then showed how the telegraph equation (206) can be recovered as an approximation to the more precise integral equation, but that the validity of this approximation breaks down in the vicinity of the diffusion fronts.

\footnotetext{
76 Boguñá et al. [28] discuss persistent random walks in higher space dimensions.

77 The result (211) may be generalized to an arbitrary number of space dimensions; cf. p. 500 in Kac's paper [367].

78 For further reading about path integral representations of the Dirac propagator, we refer to the papers of Ichinose [384,385], Jacobson and Schulman [386], Barut and Duru [387], and Gaveau and Schulman [388]; see also footnote 7 in Gaveau et al. [383] and problem 2-6, pp. 34-36 in Feynman and Hibbs [389].
} 
Similarly, the singular diffusion fronts predicted by Eq. (209) represent a source of concern if one wishes to adopt the telegraph equation (206) as a model for particle transport in a random medium. While these singularities may be acceptable in the case of photon diffusion [371-375], they seem unrealistic for massive particles, because such fronts would imply that a finite fraction of particles carries a huge amount of kinetic energy (much larger than $m c^{2}$ ). In view of these shortcomings, it appears reasonable to explore other constructions of relativistic diffusion processes [23,390]. In the next part we will discuss a different approach [23] that may provide a viable alternative to the solutions of the telegraph equation.

\subsection{Relativistic diffusion propagator}

In principle, one can distinguish two different routes towards constructing relativistic diffusion processes: One can either try to find an acceptable relativistic diffusion equation, or one can focus directly on the structure of the diffusion propagator. In the present part we shall consider the latter approach [23]. The basic idea is to rewrite the nonrelativistic diffusion propagator (199) in such a form that its relativistic generalization follows in a straightforward manner. This can be achieved be re-expressing Eq. (199) in terms of an integral-over-actions.

For this purpose, we consider a nonrelativistic particle traveling from the event $\bar{x}_{0}=\left(t_{0}, x_{0}\right)$ to $\bar{x}=(t, x)$ and assume that the particle can experience multiple scatterings on its way, and that the velocity is approximately constant between two successive scattering events. Then the total action (per mass) required along the path is given by

$$
a\left(\bar{x} \mid \bar{x}_{0}\right)=\frac{1}{2} \int_{t_{0}}^{t} \mathrm{~d} t^{\prime} v\left(t^{\prime}\right)^{2}
$$

where the velocity $v\left(t^{\prime}\right)$ is a piecewise constant function, satisfying

$$
x=x_{0}+\int_{t_{0}}^{t} \mathrm{~d} t^{\prime} v\left(t^{\prime}\right)
$$

Clearly, the nonrelativistic action (213) becomes minimal for the deterministic (direct) path, i.e., if the particle does not collide at all. In this case, it moves with constant velocity $v\left(t^{\prime}\right) \equiv\left(x-x_{0}\right) /\left(t-t_{0}\right)$ for all $t^{\prime} \in\left[t_{0}, t\right]$, yielding the smallest possible action value

$$
a_{-}\left(\bar{x} \mid \bar{x}_{0}\right)=\frac{\left(x-x_{0}\right)^{2}}{2\left(t-t_{0}\right)} .
$$

On the other hand, to match the boundary conditions it is merely required that the mean velocity equals $\left(x-x_{0}\right) /\left(t-t_{0}\right)$. Consequently, in the nonrelativistic case, the absolute velocity of a particle may become arbitrarily large during some intermediate time interval $\left[t^{\prime}, t^{\prime \prime}\right] \subset\left[t_{0}, t\right]$. Hence, the largest possible action value is $a_{+}\left(\bar{x}, \bar{x}_{0}\right)=+\infty$. These considerations put us in the position to rewrite the Wiener propagator (199) as an integral-over-actions:

$$
p\left(\bar{x} \mid \bar{x}_{0}\right) \propto \int_{a_{-}\left(\bar{x} \mid \bar{x}_{0}\right)}^{a_{+}\left(\bar{x} \mid \bar{x}_{0}\right)} \mathrm{d} a \exp \left(-\frac{a}{2 \mathscr{D}}\right),
$$

supplemented by the normalization condition

$$
1=\int \mathrm{d} x p\left(\bar{x} \mid \bar{x}_{0}\right)
$$

The representation (216) may be generalized to the relativistic case in a straightforward manner: One merely needs to insert the corresponding relativistic expressions into the boundaries of the integral (216a). A commonly considered relativistic generalization of Eq. (213), based on the particle's proper time, reads [6]

$$
a\left(\bar{x} \mid \bar{x}_{0}\right)=-\int_{t_{0}}^{t} \mathrm{~d} t^{\prime}\left[1-v\left(t^{\prime}\right)^{2}\right]^{1 / 2} .
$$

Analogous to the nonrelativistic case, the relativistic action (217) assumes its minimum $a_{-}$for the deterministic (direct) path from $x_{0}$ to $x$, characterized by a constant velocity $v\left(t^{\prime}\right) \equiv\left(x-x_{0}\right) /\left(t-t_{0}\right)$. One explicitly obtains

$$
a_{-}\left(\bar{x} \mid \bar{x}_{0}\right)=-\left[\left(t-t_{0}\right)^{2}-\left(x-x_{0}\right)^{2}\right]^{1 / 2},
$$

i.e., $a_{-}$is the negative Minkowski distance of the two spacetime events $\bar{x}_{0}$ and $\bar{x}$. The maximum action value $a_{+}=0$ is realized for particles that move at light speed. ${ }^{79}$ Hence, the transition PDF for the relativistic generalization of the Wiener process reads

$$
p\left(\bar{x} \mid \bar{x}_{0}\right)=\mathcal{N}^{-1}\left\{\exp \left[-\frac{a_{-}\left(\bar{x}, \bar{x}_{0}\right)}{2 \mathscr{D}}\right]-1\right\},
$$

if $\left(x-x_{0}\right)^{2} \leq\left(t-t_{0}\right)^{2}$, and $p\left(\bar{x} \mid \bar{x}_{0}\right) \equiv 0$ otherwise, with $a_{-}$determined by Eq. (218a).

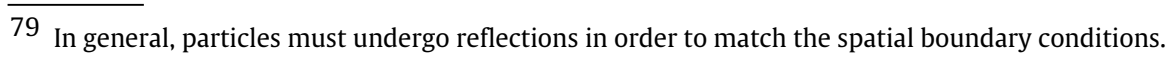




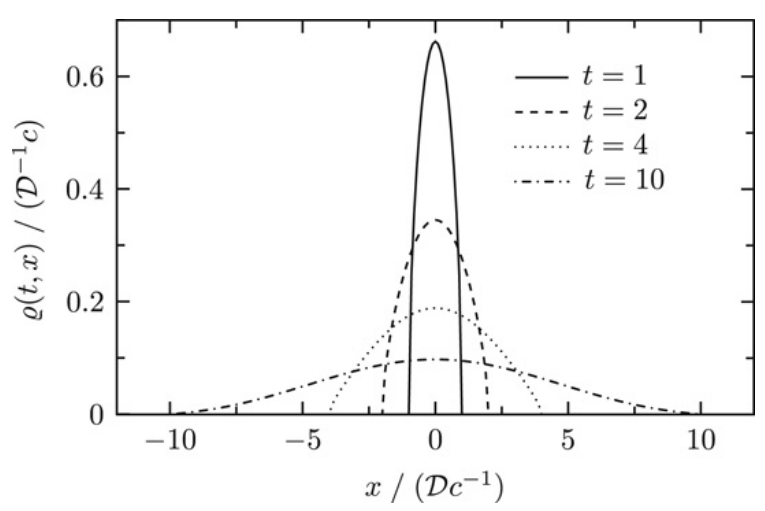

Fig. 11. Transition PDF $\varrho(t, x)=p(t, x \mid 0,0)$ for the one-dimensional $(d=1)$ relativistic diffusion process (218) at different times $t$ (measured in units of $\left.\mathscr{D} / c^{2}\right)$. At time $t=t_{0}=0$, the function $\varrho(t, x)$ reduces to a $\delta$-function centered at $x_{0}=0$. In contrast to the nonrelativistic diffusion propagator, cf. Fig. 10 , the PDF (218) vanishes outside of the light cone.

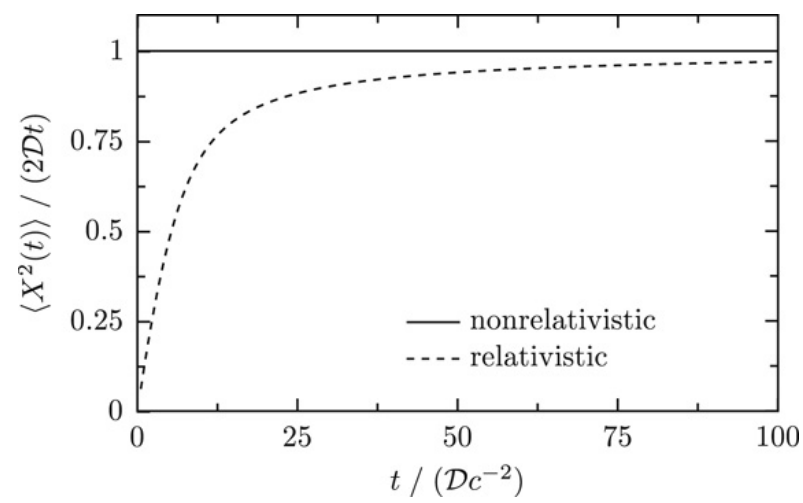

Fig. 12. Comparison of the mean square displacements $\left\langle X^{2}(t)\right\rangle$, divided by $2 \mathscr{D} t$, for the one-dimensional $(d=1)$ nonrelativistic Wiener process $(199)$ and its relativistic generalization from Eq. (218) with initial condition $\left(t_{0}, x_{0}\right)=(0,0)$.

The relativistic diffusion process described by Eq. (218) is non-Markovian, i.e., it does not fulfill Chapman-Kolmogoroff criterion (200). The functional form of the propagator (218b) remains the same for higher space dimensions $d>1$. The normalization constants $\mathcal{N}_{d}$ for $d=1,2,3$ read

$$
\mathcal{N}_{d}=\mathcal{N}_{d}^{\prime}-\frac{u^{d}}{d} O_{d},
$$

where $u:=t-t_{0}, O_{d}=2 \pi^{d / 2} / \Gamma(d / 2)$ is surface area of the $d$-dimensional unit sphere, and $\mathcal{N}_{d}^{\prime}$ can be expressed in terms of modified Bessel functions of the first kind $I_{n}$ and modified Struve functions $L_{k}$ [486], as

$$
\begin{aligned}
& \mathcal{N}_{1}^{\prime}=u \pi\left[I_{1}(\chi)+L_{-1}(\chi)\right], \\
& \mathcal{N}_{2}^{\prime}=u^{2} \frac{2 \pi}{\chi^{2}}[1+(\chi-1) \exp (\chi)], \\
& \mathcal{N}_{3}^{\prime}=u^{3} \frac{2 \pi^{2}}{\chi^{2}}\left\{\chi\left[I_{2}(\chi)+L_{0}(\chi)\right]-2 L_{1}(\chi)\right\},
\end{aligned}
$$

with $\chi=u /(2 D)$.

In contrast to the solution (209) of the telegraph equation, the propagator (218b) vanishes continuously at the diffusion fronts. Fig. 11 depicts the PDF $\varrho(t, x)=p(t, x \mid 0,0)$ of the diffusion process (218) for the one-dimensional case $d=1$ at different times $t$. The corresponding mean square displacement is plotted in Fig. 12 (dashed curve).

It is also interesting to note that the PDF (216) is a special case of a larger class of diffusion processes, defined by

$$
p_{w}\left(\bar{x} \mid \bar{x}_{0}\right)=\mathcal{N}[w]^{-1} \int_{a_{-}\left(\bar{x} \mid \bar{x}_{0}\right)}^{a_{+}\left(\bar{x} \mid \bar{x}_{0}\right)} \mathrm{d} a w(a),
$$

where $w(a) \geq 0$ is a weighting function, and $\mathcal{N}[w]$ the time-dependent normalization constant. In particular, Eq. (221) may be viewed as a path integral definition in the following sense: Physically permissible paths from $\bar{x}_{0}$ to $\bar{x}$ have action values (per mass) $a$ in the range $\left[a_{-}, a_{+}\right]$. Grouping the different paths together according to their action values, one may assign to each such class of paths, denoted by $\mathcal{C}\left(a ; \bar{x}, \bar{x}_{0}\right)$, the statistical weight $w(a)$. The integral $(221)$ can then be read as an integral 
over the equivalence classes $\mathcal{C}\left(a ; \bar{x}, \bar{x}_{0}\right)$ and their respective weights $w(a)$. The nonrelativistic Wiener process corresponds to the specific choice $w(a)=\exp [-a /(2 D)]$; hence, it appears natural to define the relativistic generalization by using the same weighting function. It is, however, worth mentioning that a very large class of functions $w(a)$ yields an asymptotic growth of the spatial mean square displacement that is proportional to $t$, corresponding to 'ordinary' diffusion. Moreover, Eq. (221) could also be used to describe super-diffusion or sub-diffusion processes [116,117,512], whose asymptotic mean square displacements grow as $t^{\alpha}, \alpha \neq 1 .{ }^{80}$

\section{Outlook}

We would like to conclude our discussion by summarizing problems which, in our opinion, deserve further consideration in the future:

- Microscopic models. The one-dimensional binary collision model from Section 4.5 appears to be the simplest example for motivating relativistic Langevin-type equations by means of an underlying microscopic model. Future investigations should focus on constructing relativistic Langevin equations from more precise particle-field interaction models; e.g., one could consider the motion of a classical relativistic point particle in quasi-static external random fields. If successful, this approach would yield more precise noise and friction models for relativistic systems ${ }^{81}$ In particular, this would contribute to clarifying under which circumstances stochastic differential equations may provide a feasible approximation to complex relativistic systems. A useful starting point for future research in this direction could be the work of Blanco et al. [416], Johnson and Hu [519-521] and Galley et al. [522], who proposed to model the interaction between quantum test particles and vacuum fluctuations of quantum fields by means of effective Langevin equations.

- Relativistic processes driven by non-Gaussian noise. The most frequently studied relativistic stochastic differential equations [11-14,17,18,20,21,24,31-34,391,394] are driven by Brownian motion (Wiener) processes which couple to the momentum coordinates. It would be interesting to also consider other driving processes (e.g., Poisson or Lévy noise) and to compare with the results of the corresponding nonrelativistic equations $[83,467,470]$. On the one hand, the properties of such generalized relativistic processes deserve to be studied from a purely mathematical point of view - e.g., with regard to potential modifications of normal or anomalous diffusion effects. On the other hand, it would be desirable to identify classes of physical systems that can be described by these processes. For example, it seems likely that the quasirandom particle acceleration [523] in various astrophysical systems may be efficiently modeled by non-Gaussian driving processes.

- Relativistic fluctuation theorems. Fluctuation theorems are mathematical relations that may be used to deduce certain thermodynamic properties of equilibrium systems by measuring suitable averages from different realizations of a nonequilibrium process [524-529]. During the past two decades, numerous fluctuation theorems have been established for various closed and open nonrelativistic systems. Both from a theoretical and practical point of view, it would be most interesting to generalize these results to the relativistic case. Recently, a first step in this direction was made by Fingerle [395], who derived a relativistic fluctuation theorem for the special relativistic Brownian motion process proposed in [17]. It will be worthwhile to extend these investigations to other, more general relativistic processes (see also Cleuren et al. [241]).

- Relativistic diffusion models in Minkowski space. The above problems refer to stochastic processes in relativistic phase space. Alternatively, one may focus on constructing and analyzing novel types of relativistic diffusion processes in Minkowski spacetime, similar to those discussed in Section 5. One particularly important issue in this context concerns the existence of reasonable path integral representations for the propagators of such processes. The latter question is closely related to the problem of finding path integral formulations of relativistic quantum propagators $[384,385,387$, 389].

- Relativistic quantum Brownian motions. Another potential generalization of the above ideas concerns the construction of relativistic quantum Brownian motion processes. Recent efforts in this direction include the aforementioned papers by Johnson and Hu [519-521] and Galley et al. [522], who considered the motion of quasi-particles in fluctuating quantum fields. Similar concepts have also been studied within the theory of stochastic semiclassical gravity, where the gravitational field plays the role of a 'Brownian particle', with the vacuum fluctuations of quantum fields forming a stochastic environment ('bath'); for details we refer to the recent review by Hu and Verdaguer [530]. Another promising starting point may be the work of Pechukas [105], and Tsonchev and Pechukas [106], who developed a simple elastic collision model of nonrelativistic quantum Brownian motions. In this context, we also mention the work of Breuer and Petruccione [531-533], who proposed a Langevin equation approach to describe quantum state diffusion in the framework of special relativity, see also Diosi [534].

- Extensions to general relativity. Last but not least, notwithstanding recent progress [395,495,498,535-538,538-540], the generalization of stochastic concepts and their applications within the framework of general relativity offers many interesting challenges for the future.

80 This can be achieved, for example, by choosing the integral boundaries as $\tilde{a}_{-}=\left(x-x_{0}\right)^{2} /\left(t-t_{0}\right)^{\alpha}, \alpha \neq 1$ and $a_{+}=\infty$, but then the variable $a$ may not be interpreted as a conventional action anymore.

81 In this context, technical and conceptual challenges are likely to arise when considering the energy loss of the particle due to radiation emission [513-518]. 


\section{Acknowledgements}

It is a great pleasure to thank our colleagues David Cubero, Fabrice Debbasch, Werner Ebeling, Igor Goychuk, Stefan Hilbert, Gert Ingold, Siegmund Kohler, Benjamin Lindner, Jesús Casado-Pascual, Lutz Schimansky-Geier, Peter Talkner, Stefan Weber and Martijn Wubs for many helpful discussions and comments.

\section{Appendix A. Stochastic integrals and calculus}

This appendix summarizes the most commonly considered stochastic integral definitions and the corresponding rules of stochastic calculus. For a more rigorous and comprehensive introduction, we refer to, e.g., Refs. [73,74,76,422].

We consider a Wiener process (standard Brownian motion) $B(t)$ as defined in Section 2.1.2; i.e., the increments $\mathrm{d} B(t):=$ $B(t+\mathrm{d} t)-B(t)$ are stochastically independent $[74,76]$ and characterized by the Gaussian distribution

$$
\mathbb{P}\{\mathrm{d} B(t) \in[y, y+\mathrm{d} y]\}=(2 \pi \mathrm{d} t)^{-1 / 2} \exp \left[-y^{2} /(2 \mathrm{~d} t)\right] \mathrm{d} y .
$$

We are interested in defining integrals of the form

$$
I=\int_{0}^{t} f(Y(s)) \odot \mathrm{d} B(s),
$$

where $f(y)$ is some real-valued function, $Y(s)$ a real-valued time-dependent process, and $\odot$ signals a discretization rule discussed below. If $B(s)$ were some ordinary differentiable function of $s \in[0, t]$, then the integral in Eq. (A.2) would simply be given by ${ }^{82}$

$$
I=\int_{0}^{t} f(Y(s)) \dot{B}(s) \mathrm{d} s,
$$

where $\dot{B}=\mathrm{d} B / \mathrm{d} s$. Unfortunately, $\dot{B}(s)$ is not well-defined for the Wiener process [74,422], but it is possible to generalize the concept of integration to also include the Wiener process and other stochastic processes [74,76,422]. However, in contrast to the standard Riemann-Stieltjes integral (A.3), the integral with respect to a stochastic process may depend on the choice of the discretization scheme $\odot$ and, in particular, also require modifications of differential calculus.

To illustrate these aspects for the most commonly considered stochastic integral definitions, we will always consider the following equidistant partition $\left\{t_{0}, t_{1}, \ldots, t_{N}\right\}$ of the time interval $[0, t]$ :

$$
\Delta t=t_{k}-t_{k-1}=t / N, \quad k=1, \ldots, N, \quad t_{0}=0, \quad t_{N}=t .
$$

\section{A.1. Ito integral}

We first summarize the properties of Ito's stochastic integral [60,61]. Its relationship to other stochastic integrals is discussed in Appendix A.4.

\section{A.1.1. One-dimensional case}

The Ito stochastic integral of some real-valued function $f(Y(t))$ with respect to a standard Brownian motion process $B(t)$ over the time-interval $[0, t]$ can be defined by

$$
\int_{0}^{t} f(Y(s)) * \mathrm{~d} B(s):=\lim _{N \rightarrow \infty} \sum_{k=0}^{N-1} f\left(Y\left(t_{k}\right)\right)\left[B\left(t_{k+1}\right)-B\left(t_{k}\right)\right],
$$

where the partition $\left\{t_{0}, \ldots, t_{N}\right\}$ is given by (A.4). The peculiar, defining feature of this integral is that, on the rhs of Eq. (A.5), the argument of the function $f$ must be evaluated at the lower boundary points $t_{k}$ of the discrete intervals $\left[t_{k}, t_{k+1}\right]$; i.e., the definition of the Ito integral is non-anticipating. Accordingly, the Ito discretization scheme is also known as the pre-point rule.

Now consider a stochastic process $Y(t)$ which, for two given functions $A(y)$ and $C(y)$, is defined by

$$
Y(t)=Y(0)+\int_{0}^{t} A(Y(s)) \mathrm{d} s+\int_{0}^{t} C(Y(s)) * \mathrm{~d} B(s),
$$

and where the last term is interpreted as an Ito integral (A.5). Stochastic integral equations like Eq. (A.6) are usually abbreviated by rewriting them as an Ito stochastic differential equation (I-SDE)

$$
\mathrm{d} Y(t)=A(Y) \mathrm{d} t+C(Y) * \mathrm{~d} B(t),
$$

\footnotetext{
82 By writing Eq. (A.3), it is implicitly assumed that $f(y), Y$ and $\dot{B}$ are sufficiently smooth functions so that this integral exists in the sense of Riemann-Stieltjes; in this case, the value of the integral (A.3) is independent of the underlying discretization scheme [76].
} 
complemented by the initial condition $Y(0)$. From the non-anticipating definition (A.5) of the Ito integral and the properties of the Wiener process it follows that [422]

83

$$
\langle C(Y) * \mathrm{~d} B(t) \mid Y(t)=y\rangle=0 .
$$

The Fokker-Planck equation for the $\operatorname{PDF} f(t, y)$ of the stochastic process defined by Eq. (A.7) reads

$$
\frac{\partial f}{\partial t}=\frac{\partial}{\partial y}\left[-A f+\frac{1}{2} \frac{\partial}{\partial y}\left(C^{2} f\right)\right]
$$

where $A=A(y)$ and $C=C(y)$. A deterministic initial condition $Y(0)=y_{0}$ translates into $f(0, y)=\delta\left(y-y_{0}\right)$.

Finally, an important peculiarity arises when one considers nonlinear transformations $G$ of the stochastic process $Y(t)$. More precisely, assuming that $Y$ is defined by the I-SDE (A.7), then the differential change of the process $Z(t):=G(Y(t))$ is given by (see, e.g., Section 4.3.2 in [422])

$$
\begin{aligned}
\mathrm{d} Z(t) & =G^{\prime}(Y) * \mathrm{~d} Y+\frac{1}{2} C(Y)^{2} G^{\prime \prime}(Y) \mathrm{d} t \\
& =\left[A(Y) G^{\prime}(Y)+\frac{1}{2} C(Y)^{2} G^{\prime \prime}(Y)\right] \mathrm{d} t+C(Y) G^{\prime}(Y) * \mathrm{~d} B(t),
\end{aligned}
$$

where $G^{\prime}(y)=\mathrm{d} G(y) / \mathrm{d} y$ and $G^{\prime \prime}(y)=\mathrm{d}^{2} G(y) / \mathrm{d} y^{2}$. Within ordinary differential calculus, the term containing $G^{\prime \prime}$ is absent. Eq. (A.10) is usually referred to as Ito formula.

\section{A.1.2. The n-dimensional case}

Consider the $n$-dimensional stochastic process $\boldsymbol{Y}(t)=\left(Y^{1}(t), \ldots, Y^{n}(t)\right)$, defined by the following $n$-dimensional generalization of Eq. (A.7):

$$
\mathrm{d} Y^{i}(t)=A^{i}(\boldsymbol{Y}) \mathrm{d} t+C_{r}^{i}(\boldsymbol{Y}) * \mathrm{~d} B^{r}(t),
$$

where $i=1, \ldots, n$ and $r=1, \ldots, K$. In Eq. (A.11), the Wiener processes $B^{r}(t)$ represent $K$ independent noise sources, and each term $C_{r}^{i}(\boldsymbol{Y}) * \mathrm{~d} B^{r}(t)$ symbolizes an Ito integral. The Fokker-Planck equation for the $\operatorname{PDF} f\left(t, y^{1}, \ldots, y^{n}\right)$ reads

$$
\frac{\partial f}{\partial t}=\frac{\partial}{\partial y^{i}}\left[-A^{i} f+\frac{1}{2} \frac{\partial}{\partial y^{j}}\left(C_{r}^{i} C_{r}^{j} f\right)\right] .
$$

The generalized Ito formula reads (see, e.g., Section 4.3.2 in [422])

$$
\mathrm{d} G[\boldsymbol{Y}(t)]=\left[A^{i} \partial_{i} G+\frac{1}{2} C^{i}{ }_{r} C_{r}^{j} \partial_{i} \partial_{j} G\right] \mathrm{d} t+C^{i}{ }_{r} \partial_{i} G * \mathrm{~d} B^{r}(t),
$$

where $\partial_{i}:=\partial / \partial y^{i}$.

\section{A.2. Stratonovich-Fisk integral}

Next we summarize the properties of an alternative stochastic integral definition which was proposed by Stratonovich [67-69] and Fisk [65,66]. In contrast to the non-anticipating Ito integral, the Stratonovich-Fisk (SF) integral is semi-anticipating, but satisfies the rules of ordinary stochastic calculus.

\section{A.2.1. One-dimensional case}

The SF stochastic integral of some real-valued function $f(Y(t))$ with respect to a standard Brownian (Wiener) motion process $B(t)$ over the time-interval $[0, t]$ can be defined by

$$
\int_{0}^{t} f(Y(s)) \circ \mathrm{d} B(s):=\lim _{N \rightarrow \infty} \sum_{k=0}^{N-1} \frac{1}{2}\left[f\left(Y\left(t_{k+1}\right)\right)+f\left(Y\left(t_{k}\right)\right)\right]\left[B\left(t_{k+1}\right)-B\left(t_{k}\right)\right]
$$

where the partition $\left\{t_{0}, \ldots, t_{N}\right\}$ is given by (A.4). In contrast to Ito's integral (A.5), the SF definition (A.14) uses the mean of the boundary values of $f$ on the intervals $\left[t_{k}, t_{k+1}\right]$; i.e., the definition of the SF integral is semi-anticipating. This discretization scheme is also known as the mid-point rule.

$\overline{83\langle\cdot| Y(t)}=y\rangle$ denotes the conditional expectation with respect to the Gaussian measure of the Wiener process $B(t)$. 
Similar to Eq. (A.6), we may consider a stochastic process $Y(t)$ defined by

$$
Y(t)=Y(0)+\int_{0}^{t} A(Y(s)) \mathrm{d} s+\int_{0}^{t} C(Y(s)) \circ \mathrm{d} B(s)
$$

where now the last term is interpreted as an SF integral (A.14). The integral equation (A.15) can be abbreviated in terms of the equivalent SF stochastic differential equation (SF-SDE)

$$
\mathrm{d} Y(t)=A(Y) \mathrm{d} t+C(Y) \circ \mathrm{d} B(t),
$$

with initial condition $Y(0)$. From the semi-anticipating definition (A.14) of the SF integral and the properties of the Wiener process it follows that [422]

$$
\langle C(Y) \circ \mathrm{d} B(t) \mid Y(t)=y\rangle=\frac{1}{2} C(y) C^{\prime}(y) \mathrm{d} t,
$$

where $C^{\prime}(y)=\mathrm{d} C(y) / \mathrm{d} y$. The Fokker-Planck equation for the PDF $f(t, y)$ of the stochastic process (A.16) reads

$$
\frac{\partial f}{\partial t}=\frac{\partial}{\partial y}\left[-A f+\frac{1}{2} C \frac{\partial}{\partial y}(C f)\right]
$$

where $A=A(y)$ and $C=C(y)$. The deterministic initial condition $Y(0)=y_{0}$ translates into $f(0, y)=\delta\left(y-y_{0}\right)$.

It can be shown [76,422] that the SF integral definition preserves the rules of ordinary stochastic calculus; i.e., if $Y(t)$ is defined by the SF-SDE (A.16), then the differential change of the process $Z(t):=G(Y(t)$ ) is given by (see, e.g., Section 4.3 .2 in [422])

$$
\begin{aligned}
\mathrm{d} Z(t) & =G^{\prime}(Y) \circ \mathrm{d} Y \\
& =A(Y) G^{\prime}(Y) \mathrm{d} t+C(Y) G^{\prime}(Y) \circ \mathrm{d} B(t),
\end{aligned}
$$

where $G^{\prime}(y)=\mathrm{d} G(y) / \mathrm{d} y$.

However, as will be discussed in Appendix A.4, for a given SF-SDE with sufficiently smooth coefficient functions $A$ and $C$ one can always find an I-SDE, which yields the same Fokker-Planck equation. Hence, in order to describe a certain physical process, one may choose that integral definition which is most convenient for the problem under consideration.

\section{A.2.2. The n-dimensional case}

Consider the $n$-dimensional stochastic process $\boldsymbol{Y}(t)=\left(Y^{1}(t), \ldots, Y^{n}(t)\right)$, defined by the following $n$-dimensional generalization of Eq. (A.16):

$$
\mathrm{d} Y^{i}(t)=A^{i}(\boldsymbol{Y}) \mathrm{d} t+C_{r}^{i}(\boldsymbol{Y}) \circ \mathrm{d} B^{r}(t)
$$

where $i=1, \ldots, n$ and $r=1, \ldots, K$. In Eq. (A.20), the Wiener processes $B^{r}(t)$ represent $K$ independent noise sources, and each term $C_{r}^{i}(\boldsymbol{Y}) \circ \mathrm{d} B^{r}(t)$ symbolizes an SF integral. The Fokker-Planck equation for the PDF $f\left(t, y^{1}, \ldots, y^{n}\right)$ reads

$$
\frac{\partial f}{\partial t}=\frac{\partial}{\partial y^{i}}\left[-\left(A^{i}+\frac{1}{2} C^{j}{ }_{r} \frac{\partial}{\partial y^{j}} C_{r}^{i}\right) f+\frac{1}{2} \frac{\partial}{\partial y^{j}}\left(C^{i}{ }_{r} C^{j}{ }_{r} f\right)\right]
$$

and the transformation rules of ordinary differential calculus apply.

\section{A.3. Backward Ito integral}

We still consider a third stochastic integral definition which is also known as the backward Ito (BI) integral [70,74]. Its relationship to the other stochastic integrals is discussed in Appendix A.4.

\section{A.3.1. One-dimensional case}

The BI stochastic integral of some real-valued function $f(Y(t))$ with respect to $B(t)$ over the time-interval [0,t] can be defined by

$$
\int_{0}^{t} f(Y(s)) \bullet \mathrm{d} B(s):=\lim _{N \rightarrow \infty} \sum_{k=0}^{N-1} f\left(Y\left(t_{k+1}\right)\right)\left[B\left(t_{k+1}\right)-B\left(t_{k}\right)\right],
$$

where the partition $\left\{t_{0}, \ldots, t_{N}\right\}$ is given by (A.4). On the rhs of Eq. (A.22), in contrast to the Ito and SF integrals, the argument of the function $f$ must be evaluated at the upper boundary points $t_{k+1}$ of the discrete intervals [ $t_{k}, t_{k+1}$ ]; i.e., the definition of this integral is anticipating. This discretization scheme is also known as the post-point rule. 
Similar to above, we may consider a stochastic process $Y(t)$ which, for two given functions $A(y)$ and $C(y)$, is defined by

$$
Y(t)=Y(0)+\int_{0}^{t} A(Y(s)) \mathrm{d} s+\int_{0}^{t} C(Y(s)) \bullet \mathrm{d} B(s),
$$

and where the last term is now interpreted as a BI integral (A.22). Equation (A.6) can be abbreviated by rewriting it as a backward Ito stochastic differential equation (BI-SDE)

$$
\mathrm{d} Y(t)=A(Y) \mathrm{d} t+C(Y) \bullet \mathrm{d} B(t),
$$

complemented by the deterministic initial condition $Y(0)$. From the anticipating definition (A.22) of the BI integral and the properties of the Wiener process, it follows that [422]

$$
\langle C(Y) \bullet \mathrm{d} B(t) \mid Y(t)=y\rangle=C(y) C^{\prime}(y) \mathrm{d} t .
$$

The Fokker-Planck equation for the $\operatorname{PDF} f(t, y)$ of the stochastic process defined by Eq. (A.24) reads

$$
\frac{\partial f}{\partial t}=\frac{\partial}{\partial y}\left[-A f+\frac{1}{2} C^{2} \frac{\partial}{\partial y} f\right],
$$

where $A=A(y)$ and $C=C(y)$. The deterministic initial condition $Y(0)=y_{0}$ translates into $f(0, y)=\delta\left(y-y_{0}\right)$.

It can be shown that, similar to the Ito integral, also the BI integral requires a modification of differential calculus. More precisely, assuming that $Y$ is defined by the BI-SDE (A.24), the differential change of the process $Z(t):=G(Y(t))$ is given by

$$
\begin{aligned}
\mathrm{d} Z(t) & =G^{\prime}(Y) \bullet \mathrm{d} Y-\frac{1}{2} C(Y)^{2} G^{\prime \prime}(Y) \mathrm{d} t \\
& =\left[A(Y) G^{\prime}(Y)-\frac{1}{2} C(Y)^{2} G^{\prime \prime}(Y)\right] \mathrm{d} t+C(Y) G^{\prime}(Y) \bullet \mathrm{d} B(t),
\end{aligned}
$$

where $G^{\prime}(y)=\mathrm{d} G(y) / \mathrm{d} y$ and $G^{\prime \prime}(y)=\mathrm{d}^{2} G(y) / \mathrm{d} y^{2}$.

\section{A.3.2. The n-dimensional case}

Consider the $n$-dimensional stochastic process $Y(t)=\left(Y^{1}(t), \ldots, Y^{n}(t)\right)$, defined by the following $n$-dimensional generalization of Eq. (A.24):

$$
\mathrm{d} Y^{i}(t)=A^{i}(\boldsymbol{Y}) \mathrm{d} t+C_{r}^{i}(\boldsymbol{Y}) \bullet \mathrm{d} B^{r}(t),
$$

where $i=1, \ldots, n$ and $r=1, \ldots, K$. In Eq. (A.28), the Wiener processes $B^{r}(t)$ represent $K$ independent noise sources, and each term $C_{r}^{i}(\boldsymbol{Y}) \bullet \mathrm{d} B^{r}(t)$ symbolizes a BI integral. The Fokker-Planck equation for the associated $\operatorname{PDF} f\left(t, y^{1}, \ldots, y^{n}\right)$ reads

$$
\frac{\partial f}{\partial t}=\frac{\partial}{\partial y^{i}}\left[-\left(A^{i}+C^{j}{ }_{r} \frac{\partial}{\partial y^{j}} C_{r}^{i}\right) f+\frac{1}{2} \frac{\partial}{\partial y^{j}}\left(C_{r}^{i} C_{r}{ }_{r} f\right)\right]
$$

The generalized backward Ito formula reads

$$
\mathrm{d} G[\boldsymbol{Y}(t)]=\left[A^{i} \partial_{i} G-\frac{1}{2} C^{i}{ }_{r} C^{j}{ }_{r} \partial_{i} \partial_{j} G\right] \mathrm{d} t+C^{i}{ }_{r} \partial_{i} G \bullet \mathrm{d} B^{r}(t),
$$

where $\partial_{i}:=\partial / \partial y^{i}$.

\section{A.4. Comparison of stochastic integrals}

As anticipated in the preceding sections, the three different stochastic integrals/SDEs may be transformed into each other. In particular, a given Fokker-Planck equation can usually be realized by any of three SDE types, upon choosing the coefficient functions appropriately. To illustrate this by example, we reconsider the $n$-dimensional SDEs from above, assuming identical noise coefficients $C_{r}^{i}$ but different drift coefficients $A_{*||_{\bullet}}^{i}(\boldsymbol{Y})$, respectively, i.e.

$$
\begin{aligned}
\mathrm{d} Y^{i}(t) & =A_{*}^{i}(\boldsymbol{Y}) \mathrm{d} t+C_{r}^{i}(\boldsymbol{Y}) * \mathrm{~d} B^{r}(t), \\
\mathrm{d} Y^{i}(t) & =A_{\circ}^{i}(\boldsymbol{Y}) \mathrm{d} t+C_{r}^{i}{ }_{r}(\boldsymbol{Y}) \circ \mathrm{d} B^{r}(t), \\
\mathrm{d} Y^{i}(t) & =A_{\bullet}^{i}(\boldsymbol{Y}) \mathrm{d} t+C_{r}^{i}(\boldsymbol{Y}) \bullet \mathrm{d} B^{r}(t),
\end{aligned}
$$


where $i=1, \ldots, n$ and $r=1, \ldots, K$. We would like to determine the drift coefficients such that these three different types of SDEs describe the same $n$-dimensional stochastic process $\boldsymbol{Y}(t)=\left(Y^{1}(t), \ldots, Y^{n}(t)\right)$ on the level of the Fokker-Planck equations ${ }^{84}$ which may be compactly summarized as follows

$$
\frac{\partial f_{\odot}}{\partial t}=\partial_{i}\left[-\left(A_{\odot}^{i}+\lambda_{\odot} C_{r}^{j} \partial_{j} C_{r}^{i}\right) f_{\odot}+\frac{1}{2} \partial_{j}\left(C_{r}^{i} C_{r}^{j} f_{\odot}\right)\right],
$$

where $\partial_{i}:=\partial / \partial y^{i}$, and $\lambda_{*}=0, \lambda_{\circ}=1 / 2$, and $\lambda_{\bullet}=1$. We distinguish three cases.

Eq. (A.31a) is given: Eq. (A.32) implies that Eqs. (A.31b) and (A.31c) describe the same process like Eq. (A.31a), if we fix

$$
A_{\circ}^{i}=A_{*}^{i}-\frac{1}{2} C^{j}{ }_{r} \partial_{j} C_{r}^{i}, \quad A_{\bullet}^{i}=A_{*}^{i}-C^{j}{ }_{r} \partial_{j} C^{i}{ }_{r} .
$$

Eq. (A.31b) is given: Eqs. (A.31a) and (A.31c) describe the same process like Eq. (A.31b), if we fix

$$
A_{*}^{i}=A_{\circ}^{i}+\frac{1}{2} C_{r}^{j}{ }_{r j} C_{r}^{i}, \quad A_{\bullet}^{i}=A_{\circ}^{i}-\frac{1}{2} C^{j}{ }_{r} \partial_{j} C^{i}{ }_{r} .
$$

Eq. (A.31c) is given: Eqs. (A.31a) and (A.31b) describe the same process like Eq. (A.31c), if we fix

$$
A_{*}^{i}=A_{\bullet}^{i}+C^{j}{ }_{r} \partial_{j} C_{r}^{i}, \quad A_{\circ}^{i}=A_{\bullet}^{i}+\frac{1}{2} C_{r}^{j}{ }_{r j} C_{r}^{i} .
$$

To summarize, by means of Eqs. (A.33), (A.34) and (A.35) one can change between the different forms of stochastic integration and stochastic differential calculus, respectively. Each SDE type has advantages and disadvantages: The Ito formalism is well suited for numerical simulations $[73,89,420]$ and yields a vanishing noise contribution to conditional expectations of the form (A.8). The Stratonovich-Fisk approach is more difficult to implement numerically, but preserves the rules of ordinary differential calculus (in contrast to Ito/backward Ito integration). Finally, within the backward Ito scheme, fluctuation dissipation relations may take a particularly elegant form (cf. Section 6.2 in Ref. [77], and Ref. [112]).

\section{A.5. Numerical integration}

A detailed introduction to the numerical simulation of SDEs can be found in [73,89,420]. A simple Monte-Carlo algorithm for numerically integrating Eq. (A.31) follows directly from the definition of the stochastic integrals. The corresponding discretization scheme, which works sufficiently well for many purposes, reads

$$
\begin{aligned}
& Y^{i}(t+\Delta t)-Y^{i}(t)=A_{*}^{i}(\boldsymbol{Y}(t)) \Delta t+C_{r}^{i}(\boldsymbol{Y}(t)) \Delta B^{r}(t), \\
& Y^{i}(t+\Delta t)-Y^{i}(t)=A_{\circ}^{i}(\boldsymbol{Y}(t)) \Delta t+\frac{1}{2}\left[C_{r}^{i}(\boldsymbol{Y}(t+\Delta t))+C_{r}^{i}(\boldsymbol{Y}(t))\right] \Delta B^{r}(t), \\
& Y^{i}(t+\Delta t)-Y^{i}(t)=A_{\bullet}^{i}(\boldsymbol{Y}(t)) \Delta t+C_{r}^{i}(\boldsymbol{Y}(t+\Delta t)) \Delta B^{r}(t) .
\end{aligned}
$$

Here, the $\Delta B^{r}(t)$ are random numbers, sampled from a Gaussian normal distribution with density

$$
\mathbb{P}\left[\Delta B^{r}(t)\right]=\left(\frac{1}{2 \pi \Delta t}\right)^{1 / 2} \exp \left\{-\frac{\left[\Delta B^{r}(t)\right]^{2}}{2 \Delta t}\right\} .
$$

As evident from Eqs. (A.36), for given functions $A_{*}^{i}$ and $C_{r}^{i}$, the discretized I-SDE (A.36a) allows for calculating the values $Y^{i}(t+\Delta t)$ directly from the preceding values $Y^{i}(t)$. By contrast, the discretized SF-SDEs (A.36b) and BI-SDEs (A.36c) are implicit equations, which must be solved for $Y^{i}(t+\Delta t)$. The latter difficulty can be avoided by transforming a given SF/BISDE to the corresponding I-SDE by means of Eqs. (A.33)-(A.35).

\section{Appendix B. Surface integrals in Minkowski spacetime}

We would like to integrate a tensorial quantity $\theta^{\mu \alpha \beta \ldots}(t, \boldsymbol{x})$ over a fixed $d$-dimensional hyperplane $\mathfrak{H}$ in $(1+d)$ dimensional Minkowski space, e.g., ${ }^{85}$

$$
g^{\alpha \beta \cdots[}[\mathfrak{H}]:=\int_{\mathfrak{H}} \mathrm{d} \sigma_{\mu} \theta^{\mu \alpha \beta \cdots}(t, \boldsymbol{x}) .
$$

\footnotetext{
84 For most practical purposes, two Markovian stochastic processes can be considered as physically equivalent if their PDFs are governed by the same Fokker-Planck equation.

85 If $\theta^{\mu \alpha \beta \ldots}$ is a tensor of rank $n$ then the quantity $g^{\alpha \beta \ldots}[\mathfrak{H}]$ from Eq. (B.1) is tensor of rank $(n-1)$.
} 
Relevant examples are integrals over the energy-momentum tensor $\theta^{\alpha \beta}$ or the current density $j^{\alpha}$, as discussed in Appendix C. We outline the general procedure for the case of $d=3$ space dimensions, considering Cartesian coordinates with metric tensor $\left(\eta_{\alpha \beta}\right)=\operatorname{diag}(-1,1,1,1)$. In this case, the surface element $\mathrm{d} \sigma_{\mu}$ may be expressed in terms of the alternating differential form [220]

$$
\mathrm{d} \sigma_{\mu}=\frac{1}{3 !} \varepsilon_{\mu \alpha \beta \gamma} \mathrm{d} x^{\alpha} \wedge \mathrm{d} x^{\beta} \wedge \mathrm{d} x^{\gamma},
$$

where $\varepsilon_{\mu \alpha \beta \gamma}$ is the Levi-Cevita tensor ${ }^{86}$ and ' $\wedge$ ' denotes the antisymmetric product

$$
\mathrm{d} x^{\alpha} \wedge \mathrm{d} x^{\beta}=-\mathrm{d} x^{\beta} \wedge \mathrm{d} x^{\alpha}
$$

implying that

$$
\mathrm{d} x^{0} \wedge \mathrm{d} x^{0}=\mathrm{d} x^{1} \wedge \mathrm{d} x^{1}=\cdots=0 .
$$

Inserting (B.2) and ordering differentials with the help of Eq. (B.3a), the integral (B.1) can be rewritten as

$$
\begin{aligned}
g^{\alpha \ldots[\mathfrak{H}]:=} & \int_{\mathfrak{H}} \mathrm{d} x^{1} \wedge \mathrm{d} x^{2} \wedge \mathrm{d} x^{3} \theta^{0 \alpha \ldots}-\int_{\mathfrak{H}} \mathrm{d} x^{0} \wedge \mathrm{d} x^{2} \wedge \mathrm{d} x^{3} \theta^{1 \alpha \ldots} \\
& +\int_{\mathfrak{H}} \mathrm{d} x^{0} \wedge \mathrm{d} x^{1} \wedge \mathrm{d} x^{3} \theta^{2 \alpha \ldots}-\int_{\mathfrak{H}} \mathrm{d} x^{0} \wedge \mathrm{d} x^{1} \wedge \mathrm{d} x^{2} \theta^{3 \alpha \ldots}
\end{aligned}
$$

With regard to thermodynamics, we are particularly interested in integrating over space-like or time-like surfaces $\mathfrak{H}$ given in the form

$$
x^{0}=t=g(\boldsymbol{x})=g\left(x^{1}, \ldots, x^{d}\right) .
$$

Typical examples are the isochronous hyperplane $\mathfrak{T}\left(\xi^{0}\right)$ of an inertial frame $\Sigma$, defined by

$$
\mathfrak{T}\left(\xi^{0}\right):=\left\{(t, \boldsymbol{x}) \mid t=\xi^{0}=: g(\boldsymbol{x})\right\},
$$

or the backward light cone $\mathfrak{C}[\mathscr{E}]$ of some spacetime event $\mathscr{E}$ with coordinates $\left(\xi^{0}, \xi\right)$ in $\Sigma$, which is given by

$$
\mathfrak{C}(\mathscr{E}):=\left\{(t, \boldsymbol{x})\left|t=\xi^{0}-\right| \boldsymbol{x}-\boldsymbol{\xi} \mid=: g(\boldsymbol{x})\right\} .
$$

Given such an explicit representation of the hyperplane, we may express the differential $\mathrm{d} x^{0}$ in Eq. (B.4) in terms of $\mathrm{d} x^{i}$ by using

$$
\mathrm{d} x^{0}=\partial_{i} g \mathrm{~d} x^{i}
$$

Inserting this expression into Eq. (B.4) and taking into account Eq. (B.3), we obtain

$$
\begin{aligned}
g^{\alpha \cdots[}[\mathfrak{H}] & =\int_{\mathfrak{H}} \mathrm{d} x^{1} \wedge \mathrm{d} x^{2} \wedge \mathrm{d} x^{3}\left[\theta^{0 \alpha \cdots}-\left(\partial_{i} g\right) \theta^{i \alpha \cdots}\right] \\
& :=\int \mathrm{d}^{d} x\left[\theta^{0 \alpha \cdots}(g(\boldsymbol{x}), \boldsymbol{x})-\left(\partial_{i} g\right) \theta^{i \alpha \cdots}(g(\boldsymbol{x}), \boldsymbol{x})\right] .
\end{aligned}
$$

Hence, in this case, we may write for the covariant surface element four-vector

$$
\left(\mathrm{d} \sigma_{\alpha}\right)=n_{\alpha} \mathrm{d}^{d} x, \quad n_{\alpha}=\left(1,-\partial_{i} g\right) .
$$

In particular, for the isochronous hyperplane $\mathfrak{T}\left(\xi^{0}\right)$ from Eq. (B.6a), we have $g(\boldsymbol{x})=\xi^{0}$ and $\partial_{i} g=0$ in $\Sigma$, yielding

$$
g^{\alpha \cdots}\left[\xi^{0}\right]:=g^{\alpha \cdots}\left[\mathfrak{T}\left(\xi^{0}\right)\right]=\int \mathrm{d}^{d} x \theta^{0 \alpha \cdots}\left(\xi^{0}, \boldsymbol{x}\right) .
$$

For comparison, when integrating over the light cone $\mathfrak{C}(\mathscr{E})$ from Eq. (B.6b), one has to use

$$
\partial_{i} g=-\frac{x^{i}-\xi^{i}}{|\boldsymbol{x}-\xi|}
$$

such that $n_{\alpha} n^{\alpha}=0$, yielding explicitly

$$
g^{\alpha \cdots}[\mathscr{E}]=\int \mathrm{d}^{d} x\left\{\theta^{0 \alpha \cdots}\left(\xi^{0}-|\boldsymbol{x}-\boldsymbol{\xi}|, \boldsymbol{x}\right)+\frac{x^{i}-\xi^{i}}{|\boldsymbol{x}-\boldsymbol{\xi}|} \theta^{i \alpha \cdots}\left(\xi^{0}-|\boldsymbol{x}-\boldsymbol{\xi}|, \boldsymbol{x}\right)\right\} .
$$

\footnotetext{
86 The total antisymmetric covariant Levi-Cevita tensor $\varepsilon_{\alpha \beta \gamma \delta}$ is 0 if two or more indicies are equal, +1 for even permutations of the indices (0123) and -1 for odd permutations. Similar to the metric tensor, the Levi-Cevita tensor is numerically invariant under Lorentz transformations with determinant +1 . For a general discussion of the properties of Levi-Cevita tensors we refer to Section 5.5 of Sexl and Urbantke [8].
} 
Example 1: Gas in a cubic container. We calculate $g^{\alpha}[\mathscr{E}]$ for the energy-momentum tensor $\theta^{\alpha \beta}$ from Eq. (106), describing an isotropic, stationary, spatially homogeneous gas in the lab frame $\Sigma$. Considering a cubic vessel $\mathbb{V}=[-L / 2, L / 2]^{d}$ in $\Sigma$, the components of $\theta^{\alpha \beta}$ in $\Sigma$ read $^{87}$

$$
\theta^{\alpha \beta}(t, \boldsymbol{x})=V^{-1} \ell(\boldsymbol{x} ; \mathbb{V}) \begin{cases}\langle\epsilon\rangle, & \alpha=\beta=0, \\ \langle\boldsymbol{p} \cdot \boldsymbol{v}\rangle / d, & \alpha=\beta=1, \ldots, d, \\ 0, & \alpha \neq \beta,\end{cases}
$$

where $V=L^{d}$. Since $\theta^{\alpha \beta}$ is diagonal and time-independent in $\Sigma$, we find

$$
g^{0}[\mathscr{E}]=\int \mathrm{d}^{d} x \theta^{00}(g(\boldsymbol{x}), \boldsymbol{x})=V^{-1}\langle\epsilon\rangle \int \mathrm{d}^{d} x \ell(\boldsymbol{x} ; \mathbb{V})=\langle\epsilon\rangle .
$$

The spatial components $g^{i}[\mathscr{E}]$ can be calculated by means of a partial integration

$$
\begin{aligned}
g^{i}[\mathscr{E}] & =-\int \mathrm{d}^{d} x\left(\partial_{i} g\right) \theta^{i i}(g, \boldsymbol{x}) \\
& =-(V d)^{-1}\langle\boldsymbol{p} \cdot \boldsymbol{v}\rangle \int \mathrm{d}^{d} x\left(\partial_{i} g\right) \ell(\boldsymbol{x} ; \mathbb{V}) \\
& =(V d)^{-1}\langle\boldsymbol{p} \cdot \boldsymbol{v}\rangle \int \mathrm{d}^{d} x g(\boldsymbol{x}) \partial_{i} \ell(\boldsymbol{x} ; \mathbb{V}) \\
& =(V d)^{-1}\langle\boldsymbol{p} \cdot \boldsymbol{v}\rangle \int \mathrm{d}^{d} x\left(\xi^{0}-|\boldsymbol{x}-\boldsymbol{\xi}|\right) \partial_{i} \ell(\boldsymbol{x} ; \mathbb{V}),
\end{aligned}
$$

where, cf. Eq. (107b),

$$
\partial_{i} \ell(\boldsymbol{x} ; \mathbb{V})=\left[\delta\left(L / 2+x^{i}\right)-\delta\left(L / 2-x^{i}\right)\right] \prod_{j \neq i} \Theta\left(L / 2+x^{j}\right) \Theta\left(L / 2-x^{j}\right)
$$

is antisymmetric with respect to $x^{i}$; hence

$$
g^{i}[\mathscr{E}]=-(V d)^{-1}\langle\boldsymbol{p} \cdot \boldsymbol{v}\rangle \int \mathrm{d}^{d} x|\boldsymbol{x}-\boldsymbol{\xi}| \partial_{i} \ell(\boldsymbol{x} ; \mathbb{V})
$$

In the one-dimensional case, where $V=L$ and $d=1$, this integral can be solved exactly, and one finds

$$
g^{1}[\mathscr{E}]=-L^{-1}\left\langle p^{1} v^{1}\right\rangle\left(\left|L / 2+\xi^{1}\right|-\left|L / 2-\xi^{1}\right|\right) .
$$

In particular, for a lab observer located in the origin $\xi^{i}=0$ we have $g^{1}[\mathscr{E}]=0$. In this example, the origin may be interpreted as the photographic center-of-mass, which can be defined by the condition $g^{1}[\mathscr{E}]=0$ in the lab frame.

If the picture is taken from outside the volume $\mathbb{V}=[-L / 2, L / 2]$, corresponding to the condition $L /\left|\xi^{1}\right| \leq 1 / 2$, then

$$
g^{1}[\mathscr{E}]=-\langle p v\rangle\left(\xi^{1} /\left|\xi^{1}\right|\right)
$$

i.e., if the mean momentum is naively sampled from a photo taken outside of the photographic center-of-mass, then even the lab observer (who is not moving with respect to the system) will find a non-vanishing momentum average value always pointing away from herself. In the case of a Jüttner gas with $\langle\boldsymbol{p} \cdot \boldsymbol{v}\rangle=d / \beta$, the magnitude of this photographic drift effect is proportional to the temperature (and particle number).

In the $d$-dimensional case, performing the $\mathrm{d} x^{i}$-integration first, we find

$$
\begin{aligned}
g^{i}[\mathscr{E}]= & \frac{\langle\boldsymbol{p} \cdot \boldsymbol{v}\rangle}{V d} \int \mathrm{d}^{d / i} x\left[\left(x^{1}-\xi^{1}\right)^{2}+\cdots\left(L / 2-\xi^{i}\right)^{2}+\cdots\right]^{1 / 2} \\
& -\frac{\langle\boldsymbol{p} \cdot \boldsymbol{v}\rangle}{V d} \int \mathrm{d}^{d / i} x\left[\left(x^{1}-\xi^{1}\right)^{2}+\cdots\left(L / 2+\xi^{i}\right)^{2}+\cdots\right]^{1 / 2},
\end{aligned}
$$

with the remaining $(d-1)$-dimensional integration (denoted by $\left.\mathrm{d}^{d / i} x\right)$ ranging over $[-L / 2, L / 2]^{d-1}$. Again, $g^{i}[\mathscr{E}]$ vanishes if $\xi^{i}=0$. In the limit $L /|\xi| \ll 1 / 2$, corresponding to an observer position far outside the volume, one may expand the integrands, yielding

$$
\begin{aligned}
g^{i}[\mathscr{E}] & \simeq-(V d)^{-1}\langle\boldsymbol{p} \cdot \boldsymbol{v}\rangle \int \mathrm{d}^{d / i} x L \xi^{i}\left[\left(x^{1}-\xi^{1}\right)^{2}+\cdots\left(\xi^{i}\right)^{2}+\cdots\right]^{-1 / 2} \\
& \simeq-(V d)^{-1}\langle\boldsymbol{p} \cdot \boldsymbol{v}\rangle\left(\xi^{i} /|\xi|\right) \int \mathrm{d}^{d / i} x L \\
& =-d^{-1}\langle\boldsymbol{p} \cdot \boldsymbol{v}\rangle\left(\xi^{i} /|\boldsymbol{\xi}|\right) .
\end{aligned}
$$

$87 \theta^{\alpha \beta}$ from Eq. (B.13) is the energy-momentum tensor per particle and has to be multiplied by the particle number $N$ to obtain the total energy and momentum of an $N$-particle system. 
In the one-dimensional case this reduces to Eq. (B.17).

Example 2: Arbitrary confinement. In the preceding example, the spatial density was taken to be piecewise constant with a singular drop-off at the boundaries (walls of the vessel). As a slightly more general example, consider an equilibrated gas in a smooth confinement - e.g., due to some external potential. Moreover, assume that there exists a preferred inertial frame $\Sigma$, where the gas is stationary with an isotropic momentum density, so that it can be described by a Lorentz-scalar one-particle density of the form

$$
\varphi(\boldsymbol{x}, \boldsymbol{p})=\varrho(\boldsymbol{x}) \phi(\boldsymbol{p}),
$$

with normalized marginal distributions $\varrho$ and $\phi$, and $\phi$ being spherically symmetric. In this case, the (kinetic) energy-momentum tensor

$$
\theta^{\alpha \beta}(t, \boldsymbol{x}):=\int \frac{\mathrm{d}^{d} p}{p^{0}} p^{\alpha} p^{\beta} \varphi(\boldsymbol{x}, \boldsymbol{p})
$$

has components

$$
\theta^{\alpha \beta}(t, \boldsymbol{x})=\varrho(\boldsymbol{x}) \begin{cases}\langle\epsilon\rangle, & \alpha=\beta=0, \\ \left\langle p^{i} v^{i}\right\rangle, & \alpha=\beta=i=1, \ldots, d, \\ 0, & \alpha \neq \beta .\end{cases}
$$

Then, from Eq. (B.12), one obtains

$$
g^{0}[\mathscr{E}]=\langle\epsilon\rangle \int \mathrm{d}^{d} x \varrho(\boldsymbol{x})=\langle\epsilon\rangle,
$$

and

$$
g^{i}[\mathscr{E}]=\left\langle p^{i} v^{i}\right\rangle \int \mathrm{d}^{d} x \frac{x^{i}-\xi^{i}}{|\boldsymbol{x}-\boldsymbol{\xi}|} \varrho(\boldsymbol{x})=: g^{i}(\boldsymbol{\xi})
$$

In this case, the photographic center-of-mass position $\xi_{*}$ in $\Sigma$ is defined by

$$
g^{i}\left(\boldsymbol{\xi}_{*}\right)=0, \quad i=1, \ldots, d ;
$$

e.g., if $\varrho$ is rotationally invariant with respect to some space point $z$ in $\Sigma$, then $\xi_{*}=\boldsymbol{z}$. On the other hand, assuming that the density $\varrho$ vanishes outside a finite region $\mathbb{V} \in \mathbb{R}^{d}$ in $\Sigma$ and that a stationary observer is located at a position $\xi$ far away from $\mathbb{V}$, then we may approximate

$$
g^{i}[\mathscr{E}] \simeq\left\langle p^{i} v^{i}\right\rangle \int \mathrm{d}^{d} x \frac{x^{i}-\xi^{i}}{|\xi|} \varrho(\boldsymbol{x})=\left\langle p^{i} v^{i}\right\rangle \frac{\left\langle x^{i}\right\rangle-\xi^{i}}{|\boldsymbol{\xi}|} .
$$

\section{Appendix C. Relativistic thermodynamics}

In this appendix, we shall refer to the Jüttner gas [188] from Section 3.2.2 in order to compare different formulations of relativistic thermodynamics $[121,122,127,150,152]$. This serves to illustrate how different definitions and conventions imply different Lorentz transformation formulas for, e.g., the temperature.

\section{C.1. Reminder: Nonrelativistic thermodynamics}

Traditionally, nonrelativistic thermodynamics intends to describe a many-particle systems by means of a few macroscopic control parameters. According to Callen [482,541], the most 'natural' candidates for thermodynamic variables are either conserved quantities (such as total internal energy $U$ in the rest frame, particle number $N$, etc.) or parameters that characterize the breaking of symmetries (e.g., the volume parameter $V$ characterizes the breaking of translation invariance, external magnetic fields may break isotropy, etc.). Within the axiomatic formulation of thermodynamics [482,541,542], one further postulates the existence of an extensive thermodynamic potential $s(U, V, \ldots)$ called entropy, required to be a monotonous function of $\mathcal{U}$. Given the entropy $\&$, intensive thermodynamic quantities (temperature $\mathcal{T}$, pressure $\mathcal{P}$, etc.) are defined by a differentiation of $s$ with respect to the extensive control parameters $(U, V, \ldots)$, yielding the first law of thermodynamics

$$
\mathrm{d} s=\mathcal{T}^{-1}(\mathrm{~d} u+\mathcal{P} \mathrm{d} V+\cdots) .
$$

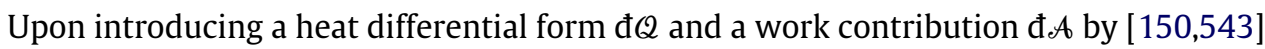

$$
\mathrm{đ} Q:=\mathcal{T} \mathrm{d} S, \quad đ \mathcal{A}:=-\mathcal{P} \mathrm{d} V-\cdots,
$$


the first law (C.1a) can be rewritten in the form

$$
\mathrm{d} Q=\mathrm{d} U-\mathrm{đ} \mathcal{A} \text {. }
$$

In the nonrelativistic framework, it is rather straightforward to generalize Eq. (C.1) to also describe moving systems [150]. To illustrate this, consider a nonrelativistic thermodynamic system of constant total mass $M$, moving at constant mean velocity $\boldsymbol{w}^{\prime}$. In this case, one can interpret the mean velocity $\boldsymbol{w}^{\prime}$ as an additional intensive variable, and define the internal energy $\mathcal{u}$ by $[150]$

$$
\varepsilon^{\prime}=U+M \boldsymbol{w}^{\prime 2} / 2,
$$

where $\mathcal{E}^{\prime}$ is the total energy of the moving system; hence,

$$
\mathrm{d} U=\mathrm{d} \mathcal{E}^{\prime}-\boldsymbol{w}^{\prime} \cdot \mathrm{d}\left(M \boldsymbol{w}^{\prime}\right)=\mathrm{d} \mathcal{E}^{\prime}-\boldsymbol{w}^{\prime} \cdot \mathrm{d} \boldsymbol{G}^{\prime},
$$

where $\boldsymbol{G}^{\prime}:=M \boldsymbol{w}^{\prime}$ is the total (mean) momentum of the system. Combining this with Eq. (C.1), the first law can be expressed as

$$
\mathrm{d} Q \mathcal{Q}=\mathrm{d} \mathscr{E}^{\prime}-\boldsymbol{w}^{\prime} \cdot \mathrm{d} \boldsymbol{G}^{\prime}-\mathrm{d} \mathcal{A},
$$

where the second term on the rhs may be interpreted as acceleration work.

\section{C.2. Relativistic case}

The relativistic generalization of Eqs. (C.1)-(C.3) is less trivial due to the following reasons [150]

- One cannot decompose the relativistic energy $\mathscr{E}$ of a thermodynamic system into the sum of a kinetic part and a term $U$ depending only on the internal state.

- In general, the rest mass (energy) of a thermodynamic system is not constant in thermodynamic processes, since, for example, any heat transfer $₫ Q \mathcal{Q}$ represents a change of energy and, therefore, of mass.

- The transfer of energy and momentum between moving systems implies the transfer of mass and, hence, of momentum.

- There exist several different, reasonable ways to define heat and work in relativistic systems.

When adopting a statistical approach ${ }^{88}$ towards relativistic thermodynamics, i.e., when identifying thermodynamic variables with statistical averages, one can add one more source of difficulty to this list [178]:

- Expectation values such as the mean energy or the mean momentum of a many-particle system are nonlocal quantities whose definition requires choosing (i.e., fixing) a specific hyperplane in spacetime. The choice of the hyperplane may single out a preferred frame of reference.

In particular, the last two aspects have led to considerable debate and confusion during the past century, e.g., concerning the behavior of temperature under Lorentz transformations [127-149,156,158-160,165,166,169,171,172,178,214,215,217, $234,476]$

In the remainder of this section, we briefly summarize three different formalisms of relativistic thermodynamics, as proposed by a Planck [121], Ott [127] and van Kampen [150,152], respectively. This serves to illustrate how different definitions and conventions yield, for example, different transformation formulas for the temperature. For this purpose, we consider a confined system with fixed particle number $N$ that, in the lab frame $\Sigma$, can be described by a spatially homogeneous, stationary Jüttner distribution (118). Aiming to identify thermodynamic quantities with statistical averages, we distinguish three different types of hyperplanes in Minkowski spacetime, as illustrated in Fig. C.1.:

We define the $\Sigma$-isochronous hyperplane $\mathfrak{T}\left(\xi^{0}\right)$, corresponding to events $(t, \boldsymbol{x})$ with $t=\xi^{0}=$ constant in the lab frame $\Sigma$, by

$$
\mathfrak{T}\left(\xi^{0}\right):=\left\{(t, \boldsymbol{x}) \mid t=\xi^{0}, \boldsymbol{x} \in \mathbb{R}^{d}\right\} .
$$

Similarly, we define for a frame $\Sigma^{\prime}$ moving at velocity $\boldsymbol{w}=(w, 0, \ldots, 0)$ relative to the lab frame $\Sigma$, the $\Sigma^{\prime}$-isochronous hyperplane $\mathfrak{T}^{\prime}\left(\xi^{\prime 0}\right)$ by

$$
\mathfrak{T}^{\prime}\left(\xi^{\prime 0}\right):=\left\{\left(t^{\prime}, \boldsymbol{x}^{\prime}\right) \mid t^{\prime}=\xi^{\prime 0}, \boldsymbol{x}^{\prime} \in \mathbb{R}^{d}\right\} .
$$

We denote the backward light cone $\mathfrak{C}(\mathscr{E})$ of some spacetime event $\mathscr{E}$, having coordinates $\bar{\xi}(\mathscr{E})=\left(\xi^{0}, \xi\right)$ in $\Sigma$ and coordinates $\left(\xi^{\prime \alpha}\right)=\left(\Lambda^{\alpha}{ }_{\beta} \xi^{\beta}\right)$ in $\Sigma^{\prime}$, by

$$
\mathfrak{C}(\mathscr{E}):=\left\{(t, \boldsymbol{x})\left|t=\xi^{0}-\right| \boldsymbol{x}-\boldsymbol{\xi} \mid, \boldsymbol{x} \in \mathbb{R}^{d}\right\} .
$$

We next introduce relativistic thermodynamic variables as integrals over these hyperplanes.

\footnotetext{
88 Eqs. (C.1) and (C.3) represent relations between macroscopic observables. The microscopic, statistical justification of these equations rests on the assumption that thermodynamic variables can be related to well-defined expectation values with respect to a phase space probability distribution [287, 492]. A statistical distribution (ensemble) with density $f$ provides a "good" model of thermodynamics if the expectation values satisfy the differential relation (C.3) for a suitable entropy functional $s[f]$. Recently, it has been shown [492] that thermodynamic relations of the type (C.1) hold for a rather wide class of distributions (not only microcanonical and canonical ones), provided the entropy functional is chosen appropriately.
} 


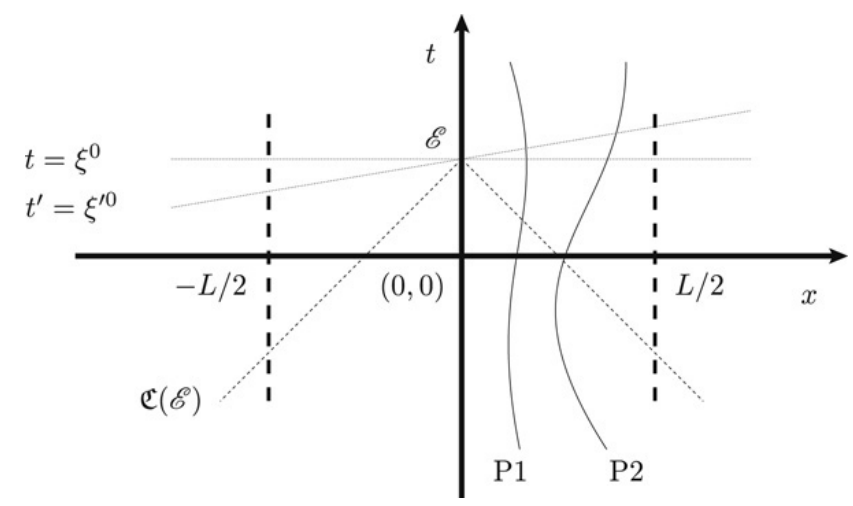

Fig. C.1. The different hyperplanes as defined in Eq. (C.4). The world lines of two particles are labeled by "P1" and "P2"; the world lines of the container walls correspond to vertical lines at $x=-L / 2$ and $x=L / 2$ (thick dashed lines), respectively. Assume a lab observer, resting at position $x=0$ in $\Sigma$, takes a photograph of the system at the spacetime event $\mathscr{E}$ with coordinates $(t, x)=\left(\xi^{0}, 0\right)$ in $\Sigma$. This photograph will reflect the state of the system along the light cone $\mathfrak{C}(\mathscr{E})$.

Energy and momentum. We distinguish energy-momentum mean values defined along the different hyperplanes ${ }^{89}$ :

$$
\begin{aligned}
& u^{\beta}\left[\xi^{0}\right]:=N \int_{\mathfrak{T}\left(\xi^{0}\right)} \mathrm{d} \sigma_{\alpha} \theta^{\alpha \beta}(t, \boldsymbol{x}), \\
& u^{\beta}\left[\xi^{\prime 0}\right]:=N \int_{\mathfrak{T}^{\prime}\left(\xi^{0}\right)} \mathrm{d} \sigma_{\alpha} \theta^{\alpha \beta}(t, \boldsymbol{x}), \\
& u^{\beta}[\mathscr{E}]:=N \int_{\mathfrak{C}(\mathscr{E})} \mathrm{d} \sigma_{\alpha} \theta^{\alpha \beta}(t, \boldsymbol{x}) .
\end{aligned}
$$

In the presence of an arbitrary external confinement, the energy-momentum tensor is not divergence-free everywhere, ${ }^{90}$ cf. Eq. (107). In this case, each of the three quantities from Eq. (C.5) represents a different ${ }^{91}$ nonlocal four-vector, since they refer to different hyperplanes, respectively. In principle, either of the three four-vectors could be used as a thermodynamic variable, giving rise to different thermodynamic formalisms [178].

The lab-isochronous four-vector $U^{\beta}\left[\xi^{0}\right]$ from Eq. (C.5a) is most easily calculated in the lab frame $\Sigma$; cf. Appendix B. Using Eq. (108c), one finds

$$
u^{\beta}\left[\xi^{0}\right]=N\left\langle p^{\beta}\right\rangle_{\xi^{0}}=N \begin{cases}\langle\epsilon\rangle, & \beta=0, \\ 0, & \beta \neq 0 .\end{cases}
$$

In the case of a Jüttner distribution, the one-particle expectation value $\langle\epsilon\rangle=\langle\epsilon\rangle_{d}$ is given by Eqs. (120b)-(120d). In another frame $\Sigma^{\prime}$, moving with velocity $w$ along the $x^{1}$-axis of $\Sigma$, one finds the transformed energy-momentum vector $\mathcal{U}^{\prime \beta}\left[\xi^{0}\right]=\Lambda(w)^{\beta}{ }_{\nu} \mathcal{U}^{v}\left[\xi^{0}\right]$ as

$$
u^{\prime \beta}\left[\xi^{0}\right]=N \begin{cases}\gamma\langle\epsilon\rangle, & \beta=0, \\ -\gamma w\langle\epsilon\rangle, & \beta=1, \\ 0, & \beta>1,\end{cases}
$$

where $\gamma(w)=\left(1-w^{2}\right)^{-1 / 2}$ and $\Lambda(w)^{\beta}{ }_{\nu}$ denotes the Lorentz transformation matrix. As discussed below, Ott [127] and van Kampen [150,152] use Eq. (C.6) as the starting point for their formulations of relativistic thermodynamics, but consider different definitions of heat and temperature, respectively.

For comparison, the $\Sigma^{\prime}$-isochronous energy-momentum four-vector, defined in Eq. (C.5b) by integration along the hyperplane $\mathfrak{T}^{\prime}\left(\xi^{0}\right)$, is most conveniently calculated in $\Sigma^{\prime}$, yielding, by virtue of Eq. (115b),

$$
\mathcal{U}^{\prime \beta}\left[\xi^{\prime 0}\right]=N\left\langle p^{\prime \beta}\right\rangle_{\xi^{\prime 0}}=N \begin{cases}\gamma\left(\langle\epsilon\rangle+w^{2}\langle\boldsymbol{p} \cdot \boldsymbol{v}\rangle / d\right), & \beta=0, \\ -\gamma w(\langle\epsilon\rangle+\langle\boldsymbol{p} \cdot \boldsymbol{v}\rangle / d), & \beta=1, \\ 0, & \beta>1,\end{cases}
$$

\footnotetext{
89 Although the energy-momentum tensor was introduced above with the help of the one-particle phase space PDF, Eqs. (C.5) can also be used to develop more general thermodynamic theories based on the energy-momentum tensor (and other conserved currents) constructed, e.g., from field theories [6,9, $164,544]$.

90 Similar problems arise if one wants to construct a relativistic continuum model for the electron [134,476,479].

91 The three four-vectors from Eq. (C.5) would coincide if the energy-momentum tensor were divergence-free everywhere [178].
} 
where $\langle\boldsymbol{p} \cdot \boldsymbol{v}\rangle=d / \beta$ for the Jüttner distribution, cf. Eq. (122). Eq. (C.7a) presents the basis of the Planck-Einstein formulation [121,122] of relativistic thermodynamics, cf. discussion below. Applying an inverse Lorentz transformation $\Lambda(-w)$ to Eq. (C.7a) gives

$$
u^{\beta}\left[\xi^{\prime 0}\right]=N \begin{cases}\langle\epsilon\rangle, & \beta=0, \\ -w\langle\boldsymbol{p} \cdot \boldsymbol{v}\rangle / d, & \beta=1, \\ 0, & \beta>1 .\end{cases}
$$

The non-vanishing component with $\beta=1$ reflects the fact that the integration was performed along the hyperplane " $t{ }^{\prime}=$ constant", which introduces an apparent asymmetry in the lab frame, cf. Fig. C.1, and results in a spurious mean momentum. ${ }^{92}$

In fact, the most frequently discussed versions of relativistic thermodynamics (see, e.g., [121,122,127-149,156,158-160, $165,166,169,171,172,178,214,215,217,234,476])$ adopt - implicitly or explicitly - either Eq. (C.6) or Eq. (C.7) as the starting point. From an experimental point of view, these equations refer to different, nonequivalent measurements procedures:

In order to determine $U^{\prime \beta}\left[\xi^{0}\right]$ a moving observer (at rest $\Sigma^{\prime}$ ) had to reconstruct the velocities and/or momentum values along the hyperplane $t=\xi^{0}$, whereas to obtain $\mathcal{U}^{\prime \beta}\left[\xi^{\prime 0}\right]$ velocities must be sampled $\Sigma^{\prime}$-simultaneously along $t^{\prime}=\xi^{\prime 0}$. On the other hand, as pointed out by Gamba [476], even for a lab observer it will be very difficult (if not impossible in practice) to reconstruct the velocity data along the hyperplane $t=\xi^{0}$ due to the finiteness of the speed of light.

Isochronous vs. light cone averages. A more natural way of performing measurements (e.g., in astronomy) is to 'take a photograph' of a given system. A photograph recorded by an observer at the event $\mathscr{E}$ reflects the state of the system along the light cone $\mathfrak{C}[\mathscr{E}]$ - see Fig. C.1. Let us assume that an idealized photograph encodes both position and velocity ${ }^{93}$ of the particles in the system. Then, the empirical energy and momentum averages which can be sampled from this data correspond to the light cone average $u^{\beta}[\mathscr{E}]$ as defined in Eq. (C.5c).

Calculating $u^{\beta}[\mathscr{E}]$ for a stationary lab distribution of the form $\varphi(\boldsymbol{x}, \boldsymbol{p})=\varrho(\boldsymbol{x}) \phi(\boldsymbol{p})$, with isotropic momentum PDF $\phi$, gives in the lab frame (cf. Appendix B)

$$
\begin{aligned}
& u^{0}[\mathscr{E}]=N\langle\epsilon\rangle, \\
& u^{i}[\mathscr{E}]=N d^{-1}\langle\boldsymbol{p} \cdot \boldsymbol{v}\rangle \int \mathrm{d}^{d} x \frac{x^{i}-\xi^{i}}{|\boldsymbol{x}-\boldsymbol{\xi}|} \varrho(\boldsymbol{x}), \quad i=1, \ldots, d,
\end{aligned}
$$

where $\left(\xi^{0}, \xi\right)$ are the spacetime coordinates $\mathscr{E}$ in $\Sigma$. By comparing with Eqs. (C.6a) and (C.7b), we see that the 0-components are identical,

$$
u^{0}[\mathscr{E}]=u^{0}\left[\xi^{0}\right]=u^{0}\left[\xi^{10}\right] ;
$$

i.e. when sampling energy values it does not matter whether this is done from a photograph or from simultaneously collected (i.e., reconstructed) data.

The situation is different, when estimating the mean momentum. As evident from Eq. (C.8b), even for a lab observer at rest in $\Sigma$, the light cone average depends on the position $\xi$ of the observer.$^{94}$ A distinguished "photographic center-of-mass" position $\boldsymbol{\xi}_{*}$ in $\Sigma$ can defined by

$$
\left.u^{i}[\mathscr{E}]\right|_{\xi=\xi_{*}}=0, \quad i=1, \ldots, d .
$$

For example, if $\varrho$ is symmetric with respect to the origin of $\Sigma$, then $\boldsymbol{\xi}_{*}=\mathbf{0}$; this would correspond to a light cone as drawn in Fig. C.1.

To illustrate how $u^{i}[\mathscr{E}]$ depends on the observer position, assume that the density $\varrho$ vanishes outside a finite region $\mathbb{V} \in \mathbb{R}^{d}$ in $\Sigma$ and that a stationary observer is located at a position $\xi$ far away from $\mathbb{V}$. In this case, one can approximate $|\boldsymbol{x}-\boldsymbol{\xi}| \simeq|\boldsymbol{\xi}|$ in the integrand of Eq. (C.8b), yielding

$$
U^{i}[\mathscr{E}]=N d^{-1}\langle\boldsymbol{p} \cdot \boldsymbol{v}\rangle \frac{\left\langle x^{i}\right\rangle-\xi^{i}}{|\boldsymbol{\xi}|} .
$$

\footnotetext{
92 Some authors $[127,151,152]$ have interpreted the $w^{2}$-term in Eq. (C.7) as an energy contribution due to presence of the walls. This view, although objected to by others [178], seems to be at least partially correct. The above derivation shows that the difference between ${\mathcal{U}^{\prime}}^{\beta}\left[\xi^{0}\right]$ and $\mathcal{u}^{\prime \beta}\left[\xi^{\prime 0}\right]$ can be attributed to the different underlying hyperplanes $\mathfrak{T}\left(\xi^{0}\right)$ and $\mathfrak{T}^{\prime}\left(\xi^{0}\right)$, respectively. However, Eqs. (C.5a) and (C.5b) would give the same result if the energy-momentum tensor were divergence-free everywhere [178]. In the above example of a homogeneous gas, the divergence is non-zero only on the boundary due to the appearance of the $\Theta$-function in the phase space density, cf. Eq. (107); i.e., the difference between $\mathcal{U}^{\prime \beta}\left[\xi^{0}\right]$ and $\mathcal{U}^{\prime \beta}\left[\xi^{\prime 0}\right]$ is indeed related to the presence of the boundary. It seems that Einstein was aware of this problem, cf. his remarks in [122], but at that time did not follow up this issue.

93 In practice, velocities can be reconstructed from color variations due the Doppler shift of spectral lines.

94 Clearly, averages in the lab frame do not depend on the specific value $\xi^{0}$ of the time coordinate if the PDF is stationary in this frame.
} 
In particular, when considering a homogeneous Jüttner gas with position mean value $\langle\boldsymbol{x}\rangle=\mathbf{0}$ and $\langle\boldsymbol{p} \cdot \boldsymbol{v}\rangle=d / \beta$, then ${ }^{95}$

$$
u^{i}[\mathscr{E}]=-\frac{\xi^{i}}{|\xi|} \frac{N}{\beta}
$$

i.e., an observer located outside of the gas, who naively estimates $u^{i}[\mathscr{E}]$ from his photographic data, could erroneously conclude that the gas is moving away from him (at a velocity proportional to the temperature). This effect ${ }^{96}$ should be taken into consideration when estimating the velocities of astrophysical objects from photographs.

From the conceptual point of view, it is worthwhile to note that the hyperplane "backward light cone $\mathfrak{C}(\mathscr{E})$ " is a relativistically invariant object which is equally accessible for any inertial observer. Put differently, if a second observer, moving relative to the first one, takes a snapshot at the same event $\mathscr{E}$ then her picture will reflect the same state of the system - even though the colors will be different due to the Doppler effect caused by the observer's relative motion [6].

Another advantage of light cone averages, as defined in Eq. (C.5c), lies in the fact that they can be generalized to general relativity $[5,6]$ in a straightforward manner, whereas it becomes very difficult to single out a globally acceptable "simultaneous" hyperplane in curved spacetime. ${ }^{97}$ In view of these benefits, light cone averages appear to be the better if not the best - suited candidates if one wishes to characterize a many-particle system by means of non-locally defined, macroscopic variables within a relativistic framework.

However, since historically most authors considered either lab-simultaneously or observer-simultaneously defined quantities, we will restrict ourselves in the remainder to discussing the implications of these two choices.

Entropy. Having identified the potential candidates for the thermodynamic state variables 'energy' and 'momentum', one still needs to specify 'entropy'. In the case of a Jüttner gas, one can define an entropy density four-current per particle by $[242,312]$

$$
s^{\alpha}(t, \boldsymbol{x})=-\int \frac{\mathrm{d}^{d} p}{p^{0}} p^{\alpha} f(t, \boldsymbol{x}, \boldsymbol{p}) \ln \left[h^{d} f(t, \boldsymbol{x}, \boldsymbol{p})\right] .
$$

The 'logarithmic' form of this entropy current is specifically adapted [492] to the exponential form of the Jüttner distribution and/or vice versa, cf. Eq. (123a). Inserting the Jüttner function (118) into Eq. (C.13), one finds that the entropy current is stationary in the lab frame $\Sigma$ and given by

$$
s^{\alpha}(t, \boldsymbol{x})=V^{-1} \ell(\boldsymbol{x} ; \mathbb{V}) \begin{cases}\ln \left(V \mathcal{Z}_{d} / h^{d}\right)+\beta\langle\epsilon\rangle_{d}, & \alpha=0, \\ 0, & \alpha=1, \ldots, d .\end{cases}
$$

Hence, the current (C.14) satisfies the conservation law

$$
\partial_{\alpha} s^{\alpha}=0 .
$$

The thermodynamic entropy $\&$ is obtained by integrating Eq. (C.14) over some space-like or light-like hyperplane $\mathfrak{H}$, yielding the Lorentz invariant quantity

$$
§[\mathfrak{H}]:=N \int_{\mathfrak{H}} \mathrm{d} \sigma_{\alpha} s^{\alpha}(t, \boldsymbol{x})=N \int_{\mathfrak{H}} \mathrm{d} \sigma_{\alpha}^{\prime} s^{\prime \alpha}\left(t^{\prime}, \boldsymbol{x}^{\prime}\right)=: \varsigma^{\prime}[\mathfrak{H}] .
$$

The conservation law (C.15) implies that the integral (C.16) is the same for the hyperplanes $\mathfrak{T}\left(\xi^{0}\right), \mathfrak{T}^{\prime}\left(\xi^{\prime 0}\right)$ and $\mathfrak{C}(\mathscr{E})$, i.e.,

$$
s[\mathscr{E}]=\varsigma^{\prime}[\mathscr{E}]=s\left[\xi^{0}\right]=\varsigma^{\prime}\left[\xi^{0}\right]=s\left[\xi^{\prime 0}\right]=\varsigma^{\prime}\left[\xi^{\prime 0}\right] .
$$

The integral (C.16) is most conveniently calculated along $\mathfrak{H}=\mathfrak{T}\left[\xi^{0}\right]$ in $\Sigma$, yielding

$$
s=N \int \mathrm{d}^{d} x s^{0}\left(\xi^{0}, \boldsymbol{x}\right)=N \ln \left(V \mathcal{Z}_{d} / h^{d}\right)+\beta N\langle\epsilon\rangle .
$$

Heat and temperature. By means of the preceding considerations, we can now summarize and compare the most commonly discussed versions of relativistic thermodynamics [121,122,127-149,156,158-160,165,166,169,171,172,178,214,215,217, $234,476]$. As mentioned above, these versions are based on simultaneously defined averages, respectively. In spite of this common feature, differences exist regarding

- the choice of the underlying hyperplane and/or

- the adopted definition of heat,

\footnotetext{
95 Note that Eqs. (C.12) and (C.8b) are consistent with Eq. (C.7b), as can be seen by letting $\xi^{1} \gg 0, \xi^{2}=\cdots=\xi^{d}=0$ and $w \rightarrow 1$ in Eq. (C.7b).

96 The effect becomes negligible if $\beta m c^{2} \gg 1$.

97 In the nonrelativistic limit case $c \rightarrow \infty$, the light cone "opens up" so that photographic measurements become isochronous in any frame in this limit.
} 
leading, for example, to different temperature transformation laws. Taking into account the historical order, we begin by recalling the Planck-Einstein formulation [121,122] of relativistic thermodynamics.

Guided by Eqs. (C.3), Planck and Einstein opted in 1907/08 for the following definition of heat \{cf. Eq. (23) in [122]\}

$$
\mathrm{d} Q^{\prime}\left(w^{\prime}\right):=\mathrm{d} \mathscr{E}^{\prime}-w^{\prime} \mathrm{d} \mathcal{g}^{\prime}+\mathcal{P}^{\prime} \mathrm{d} V^{\prime}=: \mathcal{T}^{\prime} \mathrm{d} \mathcal{S}^{\prime},
$$

where $w^{\prime}=-w$ is the constant velocity ${ }^{98}$ of the thermodynamic system in the frame $\Sigma^{\prime}$, and ${ }^{99100}$

$$
\begin{aligned}
& V^{\prime}\left(w^{\prime}\right)=V / \gamma, \\
& \mathcal{P}^{\prime}\left(w^{\prime}\right)=\mathcal{P}, \\
& \mathcal{S}^{\prime}\left(w^{\prime}\right)=\&, \\
& \mathcal{E}^{\prime}\left(w^{\prime}\right)=\gamma\left(\mathcal{E}+w^{\prime 2} \mathcal{P} V\right), \\
& \mathcal{g}^{\prime}\left(w^{\prime}\right)=\gamma w^{\prime}(\mathcal{E}+\mathcal{P} V),
\end{aligned}
$$

with $\gamma=\gamma\left(w^{\prime}\right)=\gamma(w)$ denoting the Lorentz-factor. The choice (C.19b)-(C.19f) corresponds to defining thermodynamic energy and momentum observer-simultaneously as in Eq. (C.7a) and identifying the pressure as

$$
\mathcal{P} V=N\langle\boldsymbol{p} \cdot \boldsymbol{v}\rangle / d,
$$

so that for a Jüttner gas we have

$$
\mathcal{P} V=N \beta^{-1} \text {. }
$$

Furthermore, substituting Eqs. (C.19b)-(C.19f) into Eq. (C.19a) gives

$$
\mathrm{đ} Q^{\prime}\left(w^{\prime}\right)=\gamma^{-1}(\mathrm{~d} \mathscr{E}+\mathcal{P} \mathrm{d} V)=\gamma^{-1} \mathrm{đ} Q \mathcal{Q}=\gamma^{-1} \mathcal{T} \mathrm{d} s,
$$

and comparing Eq. (C.19h) with (C.19a) leads to the temperature transformation formula of Planck [121] ${ }^{101}$

$$
\mathcal{T}^{\prime}=\gamma^{-1} \mathcal{T}=\left(1-w^{\prime 2}\right)^{1 / 2} \mathcal{T}
$$

stating that a moving body appears cooler (for the Jüttner gas $\mathcal{T}=\beta^{-1}$ ). This formalism was criticized by Ott [127] in 1963 and later also by van Kampen [150,152] and Landsberg [139,141] - mostly because the quantities $\left(\mathscr{E}^{\prime}, \mathscr{g}^{\prime}\right)$ and $(\mathscr{E}, g)=\left(\mathscr{E}^{\prime}(0), \mathscr{g}^{\prime}(0)\right)$ are not related by a Lorentz transformation. As discussed above, this drawback can be traced back to the fact that $\mathscr{E}^{\prime}$ and $\mathscr{g}^{\prime}$, if taken at different values of $w^{\prime}$, correspond to different hyperplanes, respectively [178].

To overcome this deficiency, van Kampen [150,152] proposed to define, instead of Eq. (C.19a), a thermal energy-momentum transfer four-vector by

$$
\mathrm{d} \mathcal{Q}^{\alpha}:=\mathrm{d} \mathscr{E}^{\alpha}-\mathrm{d} \mathcal{A}^{\alpha},
$$

where $\left(\mathcal{A}^{\alpha}\right):=(\mathcal{P} \mathrm{d} V, \mathbf{0})$ in the lab frame $\Sigma$, and $\mathscr{E}^{\alpha}$ is chosen to be the lab-simultaneous energy-momentum vector from Eqs. (C.6), i.e.,

$$
\mathcal{E}^{\alpha}:=\mathcal{U}^{\alpha}\left[\xi^{0}\right]
$$

The particular choice (C.20b) singles out the lab-isochronous hyperplane $\mathfrak{T}\left[\xi^{0}\right]$, and in a moving frame one then has

$$
\begin{aligned}
& \mathrm{đ}{\mathcal{Q}^{\prime}}^{\alpha}=\mathrm{d} \mathscr{E}^{\prime \alpha}-\mathrm{đ} \mathcal{A}^{\prime \alpha}, \\
& \left(\mathrm{d} \mathcal{E}^{\prime \alpha}\right)=\left(\gamma \mathcal{E}^{0}, \gamma \boldsymbol{w}^{\prime} \mathcal{E}^{0}\right), \\
& \left(đ \mathcal{A}^{\prime \alpha}\right)=\left(\gamma \mathcal{P} \mathrm{d} V, \gamma \boldsymbol{w}^{\prime} \mathcal{P} \mathrm{d} V\right),
\end{aligned}
$$

with unprimed quantities referring to the lab frame $\Sigma$, defined by $\boldsymbol{w}^{\prime}=\mathbf{0}$. While essentially agreeing on Eqs. (C.20a)-(C.20c) and also on the scalar nature of entropy $\delta^{\prime}=\delta$, van Kampen and Ott differ in how to define the temperature of a moving system, i.e., how to formulate the (first part ${ }^{102}$ of the) second law. More precisely, Ott opted for the definition

$$
\mathcal{T}^{\prime} \mathrm{d} \mathcal{s}^{\prime}:=\mathrm{đ} \mathbb{Q}^{\prime 0}=\gamma\left(\mathrm{d} \mathcal{E}^{0}+\mathcal{P} \mathrm{d} V\right)=\gamma \mathrm{đ} Q^{0}=\gamma \mathcal{T} \mathrm{d} s
$$

\footnotetext{
98 For simplicity, we again consider a thermodynamic system that moves with velocity $\boldsymbol{w}^{\prime}$ along the $x^{\prime 1}$-axis of $\Sigma^{\prime}$, so that $\boldsymbol{w}^{\prime}=\left(w^{\prime}, 0, \ldots, 0\right)=$ $(-w, 0, \ldots, 0)=-\boldsymbol{w}$ with $\boldsymbol{w}$ denoting the velocity of $\Sigma^{\prime}$ relative to the rest-frame $\Sigma$ of the system.

99 The scalar transformation (C.19c) of the pressure $\mathcal{P}$ is implied by the transformation laws of force and area [121,150].

100 After reinstating constants $c$ in Eq. (C.19f), it becomes evident that $g^{\prime}\left(w^{\prime}\right) \rightarrow w^{\prime} M$ in the nonrelativistic limit case $c \rightarrow \infty$.

101 It seems that, in the later stages of his life, Einstein changed his opinion about the correct transformation laws of thermodynamic quantities, favoring formulas which were later independently derived by Ott [127] and Arzelies [131,132]; cf. the corresponding discussion by Liu [181,182], Schröder and Treder [175], and Requardt [176].

102 The "second part" [543] of the second law states that $\mathrm{d} \& \geq 0$ in a closed system.
} 
yielding the modified temperature transformation law ${ }^{103}$

$$
\mathcal{T}^{\prime}=\gamma \mathcal{T}=\left(1-w^{\prime 2}\right)^{-1 / 2} \mathcal{T} ;
$$

i.e., according to Ott's definition of heat and temperature a moving body appears hotter.

However, van Kampen was able to demonstrate by example [150] that the Eqs. (C.21) are not well-suited if one wants to describe heat and energy-momentum exchange between systems that move at different velocities (hetero-tachic processes). To obtain a more convenient description, he proposed to characterize the heat transfer by means of the scalar quantity [150,152]

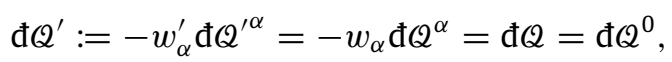

with $\left(w_{\alpha}^{\prime}\right)=\left(-\gamma, \gamma \boldsymbol{w}^{\prime}\right)$ denoting the velocity four-vector of the system in $\Sigma^{\prime}$, and reducing to $\left(w_{\alpha}\right)=(-1, \mathbf{0})$ in the lab frame $\Sigma$. He then defined the temperature by

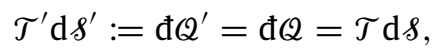

so that, in view of $s^{\prime}=\varsigma$, van Kampen's temperature is a scalar

$$
\mathcal{T}^{\prime}=\mathcal{T}
$$

i.e., according to this definition a moving body neither hotter nor colder. Adopting this temperature definition, one can define an inverse temperature four-vector by

$$
\beta_{\alpha}^{\prime}:=\mathcal{T}^{\prime-1} w_{\alpha}^{\prime}=\mathcal{T}^{-1} w_{\alpha}^{\prime},
$$

which allows us to rewrite the second law in the compact covariant form

$$
\mathrm{d}{\varsigma^{\prime}}^{\prime}=-\beta_{\alpha}^{\prime} \mathrm{d} Q^{\prime \alpha} .
$$

Thus, the thermodynamic formalisms proposed by Ott [127] and van Kampen [150,152] are based on the same labisochronous hyperplane [178] but both formulations differ only in regard to their respective temperature definition. By contrast, the Einstein-Planck formalism [121,122] is based on an observer-dependent hyperplane.

To summarize, the above discussion shows that whether a moving body appears hotter or not depends on how one defines thermodynamic quantities. Of course, before comparing the results of experimental observations with theoretical predictions, one should make sure that the applied measurement procedures are consistent with the definitions employed in the theory (choice of the hyperplane, definitions of heat and work etc.).

\section{References}

[1] A. Einstein, Über einen die Erzeugung und Verwandlung des Lichtes betreffenden heuristischen Gesichtspunkt, Ann. Phys. (Leipzig) 17 (4) (1905) $132-148$.

[2] A. Einstein, Zur Elektrodynamik bewegter Körper, Ann. Phys. (Leipzig) 17 (4) (1905) 891-921.

[3] A. Einstein, Ist die Trägheit eines Körpers von seinem Energiegehalt abhängig? Ann. Phys. (Leipzig) 18 (4) (1905) 639-641.

[4] A. Einstein, Über die von der molekularkinetischen Theorie der Wärme geforderte Bewegung von in ruhenden Flüssigkeiten suspensierten Teilchen, Ann. Phys. (Leipzig) 17 (4) (1905) 549-560.

[5] C.W. Misner, K.S. Thorne, J.A. Wheeler, Gravitation, 23rd printing, W.H. Freeman and Co., New York, 2000.

[6] S. Weinberg, Gravitation and Cosmology, John Wiley \& Sons, 1972.

[7] C. Lämmerzahl, Special relativity and Lorentz invariance, Ann. Phys. (Leipzig) 14 (1-3) (2005) 71-102.

[8] R.U. Sexl, H.K. Urbantke, Relativity, Groups, Particles, in: Springer Physics, Springer, Wien, 2001.

[9] S. Weinberg, The Quantum Theory of Fields, Vol. 1, Cambridge University Press, Cambridge, 2002.

[10] J. Masoliver, G.H. Weiss, Finite-velocity diffusion, European J. Phys. 17 (1996) 190-196.

[11] F. Debbasch, K. Mallick, J.P. Rivet, Relativistic Ornstein-Uhlenbeck process, J. Statist. Phys. 88 (1997) 945-966.

12] F. Debbasch, J.P. Rivet, A diffusion equation from the relativistic Ornstein-Uhlenbeck process, J. Statist. Phys. 90 (1998) 1179-1199.

[13] C. Barbachoux, F. Debbasch, J.P. Rivet, The spatially one-dimensional relativistic Ornstein-Uhlenbeck process in an arbitrary inertial frame, Eur. Phys. J. B 19 (2001) 37-47.

[14] C. Barbachoux, F. Debbasch, J.P. Rivet, Covariant Kolmogorov equation and entropy current for the relativistic Ornstein-Uhlenbeck process, Eur. Phys. J. B 20 (2001) 487-496.

[15] J. Angst, J. Franchi, Central limit theorem for a class of relativistic diffusions, J. Math. Phys. 48 (8) (2007) 083101.

[16] G. Chacón-Acosta, G.M. Kremer, Fokker-Planck-type equations for a simple gas and for a semirelativistic Brownian motion from a relativistic kinetic theory, Phys. Rev. E 76 (2) (2007) 021201.

[17] J. Dunkel, P. Hänggi, Theory of the relativistic Brownian motion: The (1+ 1)-dimensional case, Phys. Rev. E 71 (2005) 016124.

[18] J. Dunkel, P. Hänggi, Theory of the relativistic Brownian motion: The (1+ 3)-dimensional case, Phys. Rev. E 72 (2005) 036106.

[19] J. Dunkel, S. Hilbert, P. Hänggi, Langevin-Gleichungen mit nichtlinearer Reibung, in: T. Pöschel, H. Malchow, L. Schimansky-Geier (Eds.), Irreversible Prozesse und Selbstorganisation, Logos-Verlag, Berlin, ISBN: 3-8325-1350-7, 2006, pp. 11-21.

[20] R. Zygadło, Free Brownian motion approach to the ideal gas of relativistic particles, Phys. Lett. A 345 (2005) 323-329.

[21] J. Dunkel, P. Hänggi, Relativistic Brownian motion: From a microscopic binary collision model to the Langevin equation, Phys. Rev. E 74 (2006) 051106; Erratum Phys. Rev. E 74 (2006) 069902(E).

[22] J. Dunkel, P. Hänggi, One-dimensional nonrelativistic and relativistic Brownian motions: A microscopic collision model, Physica A 374 (2) (2007) 559-572.

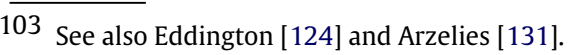


[23] J. Dunkel, P. Talkner, P. Hänggi, Relativistic diffusion processes and random walk models, Phys. Rev. D 75 (2007) 043001.

[24] K.S. Fa, Analysis of the relativistic Brownian motion in momentum space, Braz. J. Phys. 36 (3A) (2006) 777-782.

[25] B. Lindner, Exact solution for the diffusion coefficient of nonlinear Brownian motion, New J. Phys. 9 (2007) 136.

[26] C. Chevalier, F. Debbasch, Relativistic diffusions: A unifying approach, J. Math. Phys. 49 (2008) 043303.

[27] T. Koide, Microscopic derivation of causal diffusion equation using the projection operator method, Phys. Rev. E 72 (2005) 026135.

[28] M. Boguñá, J.M. Porrà, J. Masoliver, Generalization of the persistent random walk to dimensions greater than 1, Phys. Rev. E 58 (6) (1998) $6992-6998$.

[29] T. Koide, Microscopic formula of transport coefficients for causal hydrodynamics, Phys. Rev. E 75 (2007) 060103(R).

[30] J. Dunkel, P. Hänggi, S. Weber, Time parameters and Lorentz transformations of relativistic stochastic processes, Phys. Rev. E 79 (2009): 010101(R).

[31] F. Debbasch, C. Chevalier, Relativistic stochastic processes: A review, in: O. Descalzi, O.A. Rosso, H.A. Larrondo (Eds.), Medyfinol 2006: XV Conference on Nonequilibrium Statistical Mechanics and Nonlinear Physics, in: A.I.P. Conference Proceedings, vol. 913, American Institute of Physics, Melville, NY, 2007, pp. 42-48.

[32] C. Chevalier, F. Debbasch, A unifying approach to relativistic diffusions and H-theorems, Modern Phys. Lett. B 22 (2008) 383-392.

[33] I. Bailleul, Poisson boundary of a relativistic diffusion, Probab. Theory Related Fields 141 (1) (2008) 283-329.

[34] I. Bailleul, A. Raugi, Where does randomness lead in spacetime? 2008, doi:101051/ps:2008021.

[35] J. Ingen-Housz, Bemerkungen über den Gebrauch des Vergrösserungsglases, in: N.C. Molitor (Ed.), Vermischte Schriften physisch-medicinischen Inhalts, Christian Friderich Wappler, Wien, 1784.

[36] J. Ingen-Housz, Nouvelles expériences et observations sur divers objets de physique, vol. 2, Théophile Barrois le jeune, Paris, 1789, pp. 1-5.

[37] R. Brown, A brief account of microscopical observations made in the months of June, July and August, 1827, on the particles contained in the pollen of plants; and on the general existence of active molecules in organic and inorganic bodies, Philos. Mag. 4 (1828) 161-173. Reprinted in Edinburgh New Philos. J. 5 (1928) 358-371.

[38] W. Sutherland, A dynamical theory of diffusion for non-electrolytes and the molecular mass of albumin, Philos. Mag. 9 (1905) $781-785$.

[39] A. Einstein, M. von Smoluchowski, Untersuchungen über die Theorie der Brownschen Bewegung/Abhandlungen über die Brownsche Bewegung und verwandte Erscheinungen, 3rd ed., vol. 199, Harri Deutsch, Frankfurt, 1999.

[40] J. Perrin, Mouvement brownien et réalité moléculaire, Ann. Chim. Phys. 18 (1909) 5-114.

[41] P. Langevin, Sur la théorie du mouvement brownien, C. R. Acad. Sci. (Paris) 146 (1908) 530-533.

[42] D.S. Lemons, A. Gythiel, Paul Langevins's 1908 paper “On the theory of Brownian motion”, Amer. J. Phys. 65 (11) (1997) 1079-1081.

[43] A. Fokker, Die mittlere Energie rotierender elektrischer Dipole im Strahlungsfeld, Ann. Phys. 43 (4) (1914) 810-820.

[44] M. Planck, Über einen Satz der statistischen Dynamik und seine Erweiterung in der Quantentheorie, Sitzungsber. Preuss. Akad. Wiss., Berlin, 1917.

[45] O. Klein, Zur statistischen Theorie der Suspensionen und Lösungen, Ph.D. Thesis, Stockholm Högskola, 1921, published in Ark. Mat. Astron. Fys. 16(5):1-51, 1922, Almqvist \& Wiksell, Uppsala, 1921.

[46] G.E. Uhlenbeck, L.S. Ornstein, On the theory of the Brownian motion, Phys. Rev. 36 (1930) 823-841.

[47] H.A. Kramers, Brownian motion in a field of force and the diffusion model of chemical reactions, Physica 7 (4) (1940) 284-304.

[48] S. Chandrasekhar, Stochastic problems in physics and astronomy, Rev. Modern Phys. 15 (1) (1943) 1-89.

[49] M.C. Wang, G.E. Uhlenbeck, On the Theory of the Brownian Motion II, Rev. Modern Phys. 17 (1945) 323-342.

[50] L. Bachelier, Théorie de la spéculation, Ann. Sci. École Norm. Sup. 3 (17) (1900) 21-86.

[51] N. Wiener, Differential space, J. Math. Phys. 2 (1923) 131-174.

[52] N. Wiener, Generalized harmonic analysis, Acta Math. 55 (1930) 117-258.

[53] R.E.A.C. Paley, N. Wiener, A. Zygmund, Notes on random functions, Ann. of Math. 37 (1) (1933) 647-668.

[54] A. Kolmogoroff, Über die analytischen Methoden in der Wahrscheinlichkeitsrechnung, Math. Ann. 104 (4) (1931) 415-458.

[55] A. Kolmogoroff, Zur Theorie der stetigen zufälligen Prozesse, Math. Ann. 108 (1) (1933) 149-160.

[56] A. Kolmogoroff, Zur Umkehrbarkeit der statistischen Naturgesetze, Math. Ann. 113 (1) (1937) 766-772.

[57] W. Feller, Zur Theorie der stochastischen Prozesse (Existenz- und Eindeutigkeitssätze), Ann. of Math. 113 (1) (1936) 113-160.

[58] P. Lévy, Sur certains processus stochastique homogènes, Compos. Math. 7 (1939) 283-339.

[59] P. Lévy, Processus Stochastique et Mouvement Brownien, Gauthier-Villars, Paris, 1948.

[60] K. Ito, Stochastic integral, Proc. Imp. Acad. Tokyo 20 (1944) 519-524.

[61] K. Ito, On stochastic differential equations, Mem. Amer. Math. Soc. 4 (1951) 51-89.

[62] I.I. Gihman, On a method of constructing random processes, Dokl. Akad. Nauk. SSSR 58 (1947Gi) 961-964 (in Russian).

[63] I.I. Gihman, Certain differential equations with random functions, Ukrain. Math. Zh. 2 (3) (1950) 45-69 (in Russian).

[64] I.I. Gihman, On the theory of differential equations of random processes, Ukrain. Math. Zh. 2 (4) (1950) 37-63 (in Russian).

[65] D. Fisk, Quasi-martingales and stochastic integrals. Ph.D. Thesis, Michigan State University, Dept. of Statistics, 1963.

[66] D. Fisk, Quasimartingales, Trans. Amer. Math. Soc. 120 (1965) 369-389.

[67] R.L. Stratonovich, A new representation for stochastic integrals and equations, Vestnik Moskov. Univ., Ser. I: Mat., Mekh. 1 (1964) 3-12.

[68] R.L. Stratonovich, A new representation for stochastic integrals and equations, SIAM J. Control 4 (1966) 362-371.

[69] R.L. Stratonovich, Conditional Markov Processes and Their Application to the Theory of Optimal Control, American Elsevier Pub. Co., 1968.

[70] H.P. Mackean Jr., Stochastic Integrals, Academic Press, New York, 1969.

[71] E. Nelson, Theories of Brownian Motion, Princeton University Press, Princeton, 1967.

[72] D.R. Cox, H.D. Miller, The Theory of Stochastic Processes, Chapman and Hall, Oxford, 1972.

[73] H. Risken, T. Frank, The Fokker-Planck Equation: Methods of Solutions and Applications, in: Springer Series in Synergetics, Springer, Berlin, 1996.

[74] I. Karatzas, S.E. Shreve, Brownian Motion and Stochastic Calculus, 2nd edition, in: Graduate Texts in Mathematics, vol. 113, Springer, New York, Berlin, 1991.

[75] N.G. van Kampen, Stochastic Processes in Physics and Chemistry, North-Holland Personal Library, Amsterdam, 2003.

[76] M. Grigoriu, Stochastic Calculus: Applications in Science and Engineering, Birkhäuser, Boston, Basel, Berlin, 2002.

[77] P. Hänggi, H. Thomas, Stochastic processes: Time evolution, symmetries and linear response, Phys. Rep. 88 (4) (1982) 207-319.

[78] P. Hänggi, P. Talkner, M. Borkovec, Reaction rate theory: Fifty years after Kramers, Rev. Modern Phys. 62 (2) (1990) 251-341.

[79] P. Reimann, Brownian motors: Noisy transport far from equilibrium, Phys. Rep. 361 (2) (2002) 57-265.

[80] R.D. Astumian, P. Hänggi, Brownian motors, Phys. Today 55 (11) (2002) 33-39.

[81] P. Hänggi, F. Marchesoni, 100 years of Brownian motion, Chaos 15 (2005) 026101.

[82] E. Frey, K. Kroy, Brownian motion: A paradigm of soft matter and biological physics, Ann. Phys. (Leipzig) 14 (1-3) (2005) 20-50.

[83] I.M. Sokolov, J. Klafter, From diffusion to anomalous diffusion: A century after Einstein's Brownian motion, Chaos 15 (2005) 026103.

[84] W. Ebeling, I.M. Sokolov, Statistical Thermodynamics and Stochastic Theory of Nonequilibrium Systems, in: Series on Advances in Statistical Mechanics, World Scientific Publishing, Singapore, 2005.

[85] L.J.S. Allen, Stochastic Processes with Applications to Biology, Prentice-Hall, Upper Saddle River, NJ, 2002.

[86] D. Wilkinson, Stochastic Modelling for Systems Biology, in: Mathematical and Computational Biology Series, vol. 11, Chapman \& Hall/CRC, London, 2006

[87] R.J. Elliott, P.E. Kopp, Mathematics of Financial Markets, Springer-Verlag, New York, 1999.

[88] J.-P. Bouchaud, M. Potters, Theory of Financial Risks: From Statistical Physics to Risk Management, Cambridge University Press, Cambridge, 2001

[89] P. Glasserman, Monte Carlo Methods in Financial Engineering, in: Applications of Mathematics, vol. 53, Springer, New York, 2004.

[90] N.N. Bogolyubov, An elementary example of establishing statistical equilibrium in a system coupled to a thermostat, Publ. Acad. Sci. Ukr. SSR Kiev (1945) 115-137 (in Russian).

[91] V.B. Magalinskii, Dynamical model in the theory of the Brownian motion, Sov. Phys. JETP 9 (1959) 1381-1382.

[92] G.W. Ford, M. Kac, P. Mazur, Statistical Mechanics of Assemblies of Coupled Oscillators, J. Math. Phys. 6 (4) (1965) 505-515. 
[93] E. Cortes, B.J. West, K. Lindenberg, On the generalized Langevin equation: Classical and quantum mechanical, J. Chem. Phys. 82 (6) (1985) $2708-2717$.

[94] E. Pollak, Theory of activated rate processes: A new derivation of Kramers' expression, J. Chem. Phys. 85 (1986) $865-867$.

[95] A.M. Levine, M. Shapiro, E. Pollak, Hamiltonian theory for vibrational dephasing rates of small molecules in liquids, J. Chem. Phys. 88 (3) (1988) 1959-1966.

[96] P. Hänggi, Generalized Langevin equations: A useful tool for the perplexed modeller of non-equilibrium fluctuations, in: Stochastic Dynamics, in: Lecture Notes in Physics, vol. 484, Springer, Heidelberg, 1997, pp. 15-22.

[97] I.R. Senitzky, Dissipation in quantum mechanics. The harmonic oscillator, Phys. Rev. 119 (2) (1960) 670-679.

[98] I.R. Senitzky, Dissipation in quantum mechanics. The harmonic oscillator. II, Phys. Rev. 124 (3) (1961) 642-648.

[99] A.O. Caldeira, A.J. Leggett, Path integral approach to quantum Brownian motion, Physica 121A (1983) 587-616.

[100] P. Talkner, The failure of the quantum regression hypothesis, Ann. Phys. (N.Y.) 167 (2) (1986) 390-436.

[101] H. Grabert, U. Weiss, P. Talkner, Quantum theory of the damped harmonic oscillator, Z. Phys. B 55 (1984) $87-94$.

[102] A.O. Caldeira, A.J. Leggett, Influence of damping on quantum interference: An exactly soluble model, Phys. Rev. A 31 (2) (1983) $1059-1066$.

[103] G.W. Ford, M. Kac, On the quantum Langevin equation, J. Statist. Phys. 46 (5-6) (1987) 803-810.

[104] G.W. Ford, J.T. Lewis, R.F. O’Connell, Quantum Langevin equation, Phys. Rev. A 37 (11) (1988) 4419-4428.

[105] P. Pechukas, Quantum Brownian motion, in: W. Gans, A. Blumen, A. Amann (Eds.), Large-Scale Molecular Systems, in: NATO ASI B258, Plenum, New York, 1991, p. 123.

[106] S. Tsonchev, P. Pechukas, Binary collision model for quantum Brownian motion, Phys. Rev. E 61 (2000) 6171-6182.

[107] S. Kohler, J. Lehmann, P. Hänggi, Driven quantum transport on the nanoscale, Phys. Rep. 406 (2005) $379-443$.

[108] P. Hänggi, G.L. Ingold, Fundamental aspects of quantum Brownian motion, Chaos 15 (2005) 026105.

[109] P. Hänggi, G.L. Ingold, Quantum Brownian motion and the third law of thermodynamics, Acta Phys. Polon. B 37 (5) (2006) $1537-1549$.

[110] P. Riseborough, P. Hänggi, U. Weiss, Exact results for a damped quantum mechanical harmonic oscillator, Phys. Rev. A 31 (1985) 471-478.

[111] R.F. Fox, Gaussian stochastic processes in physics, Phys. Rep. 48 (3) (1978) 179-283.

[112] Y.L. Klimontovich, Nonlinear Brownian motion, Phys.-Uspekhi 37 (8) (1994) 737-766.

[113] Y.L. Klimontovich, Statistical Theory of Open Systems, Kluwer Acad. Publ., Dordrecht, 1995.

[114] P. Hänggi, P. Jung, Colored noise in dynamical systems, Adv. Chem. Phys. 89 (1995) 239-326

[115] Y. Abe, S. Ayik, P.G. Reinhard, E. Suraud, On stochastic approaches of nuclear dynamics, Phys. Rep. 275 (2-3) (1996) $49-196$.

[116] J.-P. Bouchaud, A. Georges, Anomalous diffusion in disordered media: Statistical mechanisms, models and physical applications, Phys. Rep. 195 (4-5) (1990) 127-293.

[117] R. Metzler, J. Klafter, The random walk's guide to anomalous diffusion: A fractional dynamics approach, Phys. Rep. 339 (1) (2000) 1-77.

[118] H. Grabert, P. Schramm, G.-L. Ingold, Quantum brownian motion: The functional integral approach, Phys. Rep. 168 (3) (1988) $115-207$.

[119] M. Grifoni, P. Hänggi, Driven quantum tunneling, Phys. Rep. 304 (1998) 229-354.

[120] M. Planck, Zur Dynamik bewegter Systeme, Sitzungsber. Preuss. Akad. Wiss., Berlin, 1907, pp. 542-570.

[121] M. Planck, Zur Dynamik bewegter Systeme, Ann. Phys. (Leipzig) 26 (1908) 1-34.

[122] A. Einstein, Über das Relativitätsprinzip und die aus demselben gezogenen Folgerungen, Jahrbuch Radioaktivität Elektron. 4 (1907) $411-462$.

[123] W. Pauli, Relativitätstheorie, in: Encyklopädie der mathematischen Wissenschaften mit Einschluss ihrer Anwendungen, vol. V/2, Teubner, Leipzig, 1921, 539-775.

[124] A.S. Eddington, The Mathematical Theory of Relativity, University Press Cambridge, 1923.

[125] R.C. Tolman, Relativity Thermodynamics and Cosmology, Clarendon Press, Oxford, 1934.

[126] D. Van Dantzig, On the phenomenological thermodynamics of moving matter, Physica 6 (1939) 673-704.

[127] H. Ott, Lorentz-Transformation der Wärme und der Temperatur, Z. Phys. 175 (1963) 70-104.

[128] A. Børs, Note on the temperature of a gas moving at relativistic speed, Proc. Roy. Soc. (London) 86 (1965) 1141-1143.

[129] A. Gamba, Relativistic transformations of thermodynamical quantities, Nuovo Cimento 37 (4) (1965) 1792-1794.

[130] W.G. Sutcliffe, Lorentz transformations of thermodynamic quantities, Nuovo Cimento B 9 (1965) 683-686.

[131] H. Arzelies, Transformation relativiste de la temperature et de quelques autres grandeurs thermodynamiques, Nuovo Cimento 35 (1965) $792-804$.

[132] H. Arzelies, Sur le concept de temperature en thermodynamique relativiste et en thermodynamique statistique, Nuovo Cimento B 40 (2) (1965) $333-344$.

[133] H. Arzelies, Comment on Dr. Kibble's article, Nuovo Cimento B 41 (1) (1966) 81-82.

[134] F. Rohrlich, True and apparent transformations, classical electrons and relativistic thermodynamics, Nuovo Cimento B 45 (1966) $76-83$.

[135] P.T. Landsberg, Special relativistic thermodynamics, Proc. Phys. Soc. 89 (1966) 1007-1016.

[136] T.W.B. Kibble, Relativistic transformation laws for thermodynamic variables, Nuovo Cimento B 41 (1966) $72-85$

[137] R.K. Pathria, Lorentz transformation of thermodynamic quantities, Proc. Phys. Soc. (London) 88 (1966) 791-799.

[138] R.K. Pathria, Lorentz transformation of thermodynamic quantities. II, Proc. Phys. Soc. (London) 91 (1966) 1-7.

[139] P.T. Landsberg, Does a moving body appear cool? Nature 212 (1966) 571-572.

[140] J.H. Fremlin, Does a moving body appear cool? Nature 213 (1967) 277.

[141] P.T. Landsberg, Does a moving body appear cool? Nature 214 (1967) 903-904.

[142] P.D. Noerdlinger, A moving body must "appear" cool, Nature 213 (1967) 1117.

[143] I.P. Williams, Temperature of a moving body, Nature 213 (1967) 1118

[144] I.P. Williams, Does a moving body appear cooler, Nature 214 (1967) 1105.

[145] J.L. Redding, Temperature of a moving body, Nature 215 (1967) 1160-1161.

[146] C. Møller, Relativistic thermodynamics. A strange incident in the history of physics, Kgl. Dan. Vidensk. Selsk., Mat.-Fys. Medd. 36 (1) (1967) 1-27.

[147] J. Lindhard, Temperature in special relativity, Physica 38 (4) (1968) 635-640.

[148] A.G. Georgiu, Special relativity and thermodynamics, Proc. Cambridge Philos. Soc. 66 (1966) 423-429.

[149] P.T. Landsberg, K.A. Johns, The problem of moving thermometers, Proc. Roy. Soc. (London) 306 (1478) (1968) 477-486.

[150] N.G. van Kampen, Relativistic thermodynamics of moving systems, Phys. Rev. 173 (1968) 295-301.

[151] N.G. van Kampen, Lorentz-invariance of the distribution in phase space, Physica 43 (1969) 244-262.

[152] N.G. van Kampen, Relativistic thermodynamics, J. Phys. Soc. Japan Suppl. 26 (1969) 316-321.

[153] N.G. van Kampen, A model for relativistic heat transport, Physica 46 (1970) 315-332.

[154] I. Müller, Toward relativistic thermodynamics, Arch. Ration. Mech. Anal. 34 (1969) 259-282.

[155] J.L. Lebowitz, N.G. van Kampen, J.E. Mayer, J.L. Jackson, Discussion of relativistic thermodynamics, J. Phys. Soc. Japan Suppl. 26 (1969) 321.

[156] V.H. Hamity, Relativistic thermodynamics, Phys. Rev. 187 (1969) 1745-1752.

[157] G. Horwitz, Rest frames in relativistic thermodynamics, Phys. Rev. D 4 (12) (1971) 3812-3813.

[158] H. Callen, G. Horwitz, Relativistic thermodynamics, Amer. J. Phys. 39 (1971) 938-947.

[159] A.G. Georgiu, A relativistic form of statistical thermodynamics, Internat. J. Theoret. Phys. 3 (1) (1970) $41-45$

[160] P.T. Landsberg, K.A. Johns, The Lorentz transformation of heat and work, Ann. Phys. 56 (1970) 299-318.

[161] A. Jabs, Lorentz-invariant and non-invariant momentum space and thermodynamics, Nuclear. Phys. B 34 (1) (1971) $177-188$.

[162] Ø Grøn, Asynchronous formulation of relativistic statics and thermodynamics, Nuovo Cimento B 17 (1) (1973) $141-165$.

[163] D. Eimerl, On relativistic thermodynamics, Ann. Phys. 91 (2) (1975) 481-498.

[164] W. Israel, Nonstationary irreversible thermodynamics: A causal relativistic theory, Ann. Phys. 100 (1-2) (1976) 310-331.

[165] N. Agmon, Relativistic transformations of thermodynamic quantities, Found. Phys. 7 (5-6) (1977) $331-339$.

[166] J.E. Krizan, Temperature and relativistic thermodynamics, Phys. Lett. A 71 (2-3) (1979) 174-176.

[167] W. Israel, J.M. Stewart, Transient relativistic thermodynamics and kinetic theory, Ann. Phys. 118 (2) (1979). 
[168] P.T. Landsberg, Thought experiment to determine the special relativistic temperature transformation, Phys. Rev. Lett. 45 (3) (1980) 149-150.

[169] P.T. Landsberg, Einstein and statistical thermodynamics. I. Relativistic thermodynamics, European J. Phys. 2 (1981) $203-207$.

[170] I-Shih Liu, I. Müller, T. Ruggeri, Relativistic thermodynamics of gases, Ann. Phys. (NY) 169 (1986) $191-219$.

[171] A. Komar, Relativistic Temperature, Gen. Relativity Gravitation 27 (11) (1995) 1185-1206.

[172] P.T. Landsberg, G.E.A. Matsas, Laying the ghost of the relativistic temperature transformation, Phys. Lett. A 223 (1996) 401-403.

[173] G.M. Kremer, I. Müller, Dynamic pressure in relativistic thermodynamics, Ann. Inst. H. Poincaré 67 (1997) 111-121.

[174] J. Rembielinski, K.A. Smolinski, G. Duniec, Thermodynamics and preferred frame, Found. Phys. Lett. 14 (2001) 487-500.

[175] W. Schröder, H.J. Treder, The Einstein-Laue discussion, British J. Hist. Sci. 27 (92) (1992) 113-114.

[176] M. Requardt, Thermodynamics meets special relativity - Or what is real in physics, 2008. arXiv:0801.2639v1 [gr-qc].

[177] W. Israel, Covariant fluid mechanics and thermodynamics: An introduction, in: A. Anile, Y. Choquet-Bruhat (Eds.), Relativistic Fluid Dynamics, in: Lecture Notes in Mathematics, vol. 1385, Springer-Verlag, Berlin, 1987.

[178] C.K. Yuen, Lorentz transformation of thermodynamic quantities, Amer. J. Phys. 38 (1970) $246-252$.

[179] D. Ter Haar, H. Wegeland, Thermodynamics and statistical mechanics on the special theory of relativity, Phys. Rep. 1 (2) (1971) 31-54.

[180] G. Neugebauer, Relativistische Thermodynamik, Reihe Wissenschaft. Friedr. Vieweg \& Sohn, Wiesbaden/Braunschweig, 1981.

[181] C. Liu, Einstein and relativistic thermodynamics, British J. Hist. Sci. 25 (1992) 185-206.

[182] C. Liu, Is there a relativistic thermodynamics? A case study in the meaning of special relativity, Stud. Hist. Philos. Sci. 25 (1994) $983-1004$.

[183] I. Müller, Speeds of propagation in classical and relativistic extended thermodynamics, Living Rev. Relativ. 2 (1) (1999) http://www.livingreviews.org/lrr-1999-1.

[184] K. von Mosengeil, Theorie der stationären Strahlung in einem gleichförmig bewegten Hohlraum, Ann. Phys. (Leipzig) 22 (1907) 867-904.

[185] M. von Laue, Zur Dynamik der Relativitätstheorie, Ann. Phys. (Leipzig) 35 (1911) 524-542.

[186] F. Jüttner, Das Maxwellsche Gesetz der Geschwindigkeitsverteilung in der Relativtheorie, Ann. Phys. (Leipzig) 34 (5) (1911) 856-882.

[187] J.C. Maxwell, On the dynamical theory of gases, Philos. Trans. R. Soc. Lond. 157 (1867) 49-88.

[188] J.L. Synge, The Relativistic Gas, North-Holland, Amsterdam, 1957.

[189] R.C. Tolman, A general theory of energy partition with applications to quantum theory, Phys. Rev. 11 (4)(1918) $261-275$.

[190] H. Einbinder, Generalized virial theorems, Phys. Rev. 74 (7) (1948) 803-805.

[191] J.E. Krizan, Matter and radiation in equilibrium, Phys. Rev. D 31 (8) (1985) 1968-1971

[192] W. Lucha, F.F. Schöberl, Relativistic virial theorem, Phys. Rev. Lett. 64 (23) (1990) 2733-2735.

[193] A. Komar, Relativistic equipartition, Gen. Relativity Gravitation 28 (4) (1995) 379-385.

[194] F. Jüttner, Die relativistische Quantentheorie des idealen Gases, Z. Phys. 47 (1928) 542-566

[195] W.C. Schieve, Covariant relativistic statistical mechanics of many particles, Found. Phys. 35 (8) (2005) 1359-1381.

[196] E. Lehmann, Covariant equilibrium statistical mechanics, J. Math. Phys. 47 (2006) 023303.

[197] G. Kaniadakis, Towards a relativistic statistical theory, Physica A 365 (2006) 17-23.

[198] L.P. Horwitz, W.C. Schieve, C. Piron, Gibbs ensembles in relativistic classical and quantum mechanics, Ann. Phys. 137 (1981) 306-340.

[199] L.P. Horwitz, S. Shashoua, W.C. Schieve, A manifestly covariant relativistic Boltzmann equation for the evolution of a system of events, Physica A 161 (1989) 300-338.

[200] L. Burakovsky, L.P. Horwitz, Generalized Boltzmann equation in a manifestly covariant relativistic statistical mechanics, Found. Phys. 25 (9) (1995) $1335-1358$

[201] A. Aliano, L. Rondoni, G.P. Moriss, Maxwell-Jüttner distributions in relativistic molecular dynamics, Eur. Phys. J. B 50 (2006) $361-365$.

[202] D. Cubero, J. Casado-Pascual, J. Dunkel, P. Talkner, P. Hänggi, Thermal equilibrium and statistical thermometers in special relativity, Phys. Rev. Lett. 99 (2007) 170601

[203] P.G. Bergmann, Generalized statistical mechanics, Phys. Rev. 84 (5) (1951) 1026-1033.

[204] A.E. Scheidegger, R.V. Krotkov, Relativistic statistical thermodynamics, Phys. Rev. 89 (5) (1953) 1096-1100.

[205] J.E. Krizan, P. Havas, Relativistic corrections in the statistical mechanics of interacting particles, Phys. Rev. 128 (6) (1962) $2916-2924$

[206] N.A. Chernikov, Equilibrium distribution of the relativistic gas, Acta Phys. Polon. 26 (1964) 1069-1092.

[207] C. Møller, Gibbs' statistical mechanics in the theory of relativity, Kgl. Dan. Vidensk. Selsk., Mat.-Fys. Medd. 36 (16) (1968) 1-44.

[208] J.H. Eberly, A. Kujawski, Relativistic statistical mechanics and blackbody radiation, Phys. Rev. 155 (1967) 10-19.

[209] R. Balescu, On the statistical mechanics of a relativistic quantum plasma, Physica 31 (11) (1965) 1599-1602.

[210] R. Balescu, Velocity inversion in statistical mechanics, Physica 36 (1967) 433-456.

[211] R. Balescu, T. Kotera, On the covariant formulation of classical relativistic statistical mechanics, Physica 33 (3) (1967) 558-580.

[212] R. Balescu, T. Kotera, E. Piña, Lorentz transformations in phase space and in physical space, Physica 33 (3) (1967) $581-594$.

[213] R. Hakim, Relativistic perfect gas in an external force field, Phys. Rev. 162 (1967) 128-133.

[214] R. Balescu, Relativistic statistical thermodynamics, Physica 40 (3) (1968) 309-338.

[215] R. Balescu, On relativistic statistical thermodynamics, J. Phys. Soc. Japan Suppl. 26 (1969) 313-315.

[216] R. Balescu, L. Brenig, Relativistic covariance of non-equilibrium statistical mechanics, Physica 54 (4) (1971) 504-521.

[217] S. Nakajima, On relativistic statistical thermodynamics, Progr. Theoret. Phys. 41 (6) (1968) 1450-1460.

[218] K.A. Johns, P.T. Landsberg, Ergodicity and the Lorentz transformation of time-based probabilities, J. Phys. A 3 (1970) 113-120.

[219] K.A. Johns, P.T. Landsberg, Maximum entropy arguments in relativistic statistical mechanics, J. Phys. A 3 (1970) 121-127.

[220] R. Hakim, Remarks on relativistic statistical mechanics. I, J. Math. Phys. 8 (6) (1965) 1315-1344.

[221] R. Hakim, Remarks on relativistic statistical mechanics. II. Hierarchies for the reduced densities, J. Math. Phys. 8 (7) (1965) 1397-1400.

[222] R. Hakim, Sur la mécanique statistique relativiste, Ann. Inst. H. Poincaré (A) 6 (3) (1967) 225-244

[223] E.A. Remler, Some connections between relativistic classical mechanics, statistical mechanics, and quantum field theory, Phys. Rev. D 16 (12) (1977) 3464-3473.

[224] R. Hakim, Statistical mechanics of relativistic dense matter systems, Riv. Nuovo Cimento 1 (6) (1978) 1-52.

[225] C. Cercignani, Speed of propagation of infinitesimal disturbances in a relativistic gas, Phys. Rev. Lett. 50 (15) (1983) $1122-1124$.

[226] U. Ben Ya'acov, Lorentz symmetry of subdynamics in relativistic systems, Physica A 222 (1995) 307-329.

[227] T. Matolsci, J. Kristóf, M. Székely, On the momentum distribution of molecules of an ideal gas, Publ. Appl. Anal. 7 (1996) 1-14.

[228] V. Tretyak, On relativistic models in the equilibrium statistical mechanics, Condens. Mater. Phys. 1 (1998) 553-568.

[229] A. Duviryuk, A. Nazarenko, V. Tretyak, Classical relativistic system of N charges. Hamiltonian description, form of dynamics, and partition function, Condens. Matter Phys. 4 (2001) 5-14.

[230] F. Debbasch, J.P. Rivet, W.A. van Leeuwen, Invariance of the relativistic one-particle distribution function, Physica A 301 (2001) 181-195.

[231] M. Montesinos, C. Rovelli, Statistical mechanics of generally covariant quantum theories: A boltzmann-like approach, Classical Quantum Gravity 18 (2001) 555-569.

[232] G. Kaniadakis, Statistical mechanics in the context of special relativity, Phys. Rev. E 66 (2002) 056125.

[233] C. Beck, Generalized statistical mechanics of cosmic rays, Physica A 331 (1-2)(2004) 173-181.

[234] G. Ares de Parga, B. López-Carrera, F. Angulo-Brown, A proposal for relativistic transformations in thermodynamics, J. Phys. A 38 (2005) 2821-2834.

[235] R. Silva, J.A.S. Lima, Relativity, nonextensivity and extended power law distributions, Phys. Rev. E 72 (2005) 057101.

[236] G. Kaniadakis, Statistical mechanics in the context of special relativity. ii, Phys. Rev. E 72 (2005) 036108.

[237] R. Silva, The relativistic statistical theory and Kaniadakis entropy: An approach through a molecular chaos hypothesis, Eur. Phys. J. B 54 (4) (2006) 499-502.

[238] F. Becattini, L. Ferroni, The microcanonical ensemble of the ideal relativistic quantum gas, Eur. Phys. J. C 51 (2007) 899-912. 
[239] G. Lina, D. Jiulin, L. Zhipeng, The property of kappa-deformed statistics for a relativistic gas in an electromagnetic field: Kappa parameter and kappadistribution, Phys. Lett. A 367 (2007) 431-435.

[240] T.K. Nakamura, Covariant thermodynamics of an object of finite volume, Phys. Lett. A 352 (2006) 175-177.

[241] B. Cleuren, K. Willaert, A. Engel, C. Van den Broeck, Fluctuation theorem for entropy production during effusion of a relativistic ideal gas, Phys. Rev. E 77 (2) (2008) 022103.

[242] F. Debbasch, Equilibrium distribution function of a relativistic dilute perfect gas, Physica A 387 (11) (2007) 2443-2454.

[243] A.D. Fokker, Ein invarianter Variationssatz fr die Bewegung mehrerer elektrischer Massenteilchen, Z. Phys. A 58 (5-6) (1929) $386-393$.

[244] J.A. Wheeler, R.P. Feynman, Interaction with the absorber as the mechanism of radiation, Rev. Modern Phys. 17 (2-3) (1945) 157-181.

[245] J.A. Wheeler, R.P. Feynman, Classical electrodynamics in terms of direct interparticle action, Rev. Modern Phys. 21 (3) (1949) $425-433$.

[246] M.H.L. Pryce, The mass-centre in the restricted theory of relativity and its connexion with the quantum theory of elementary particles, Proc. R. Soc. Lond. 195 (1040) (1948) 62-81.

[247] P. Havas, Four-dimensional formulations of Newtonian mechanics and their relation to the special and the general theory of relativity, Rev. Modern Phys. 36 (4) (1964) 938-965.

[248] P. Havas, J.N. Goldberg, Lorentz-invariant equations of motion of point masses in the general theory of relativity, Phys. Rev. 128 (1) (1962) $398-414$.

[249] H. Van Dam, E.P. Wigner, Classical relativistic mechanics of interacting point particles, Phys. Rev. 138 (6B) (1965) B1576-B1582.

[250] H. Van Dam, E.P. Wigner, Instantaneous and asymptotic conservation laws for classical relativistic mechanics of interacting point particles, Phys. Rev. 142 (4) (1966) 838-843.

[251] D.G. Currie, T.F. Jordan, E.C.G. Sudarshan, Relativistic invariance and Hamiltonian theories of interacting particles, Rev. Modern Phys. 35 (2) (1963) $350-375$.

[252] H. Leutwyler, A no interaction theorem in classical relativistic Hamiltonian particle mechanics, Nuovo Cimento 37 (1965) 556-567.

[253] G. Marmo, N. Mukunda, E.C.G. Sudarshan, Relativistic particle dynamics - Lagrangian proof of the no-interaction theorem, Phys. Rev. D 30 (10) (1984) $2110-2116$.

[254] R. Arens, D.G. Babbitt, The geometry of relativistic n-particle interactions, Pacific J. Math. 28 (2) (1969).

[255] C.K. Chou, The Vlasov equations, Technical Report NASA-CR-74673; E20-151, 19651101, NASA Center for AeroSpace Information (CASI), 1965. Available from http://ntrs.nasa.gov/.

[256] P. Havas, J. Stachel, Invariances of approximately relativistic Lagrangians and the center-of-mass theorem. i, Phys. Rev. 185 (5) (1969) $1636-1647$.

[257] H.W. Woodcock, P. Havas, Approximately relativistic Lagrangians for classical interacting point particles, Phys. Rev. D 6 (12) (1972) $3422-3444$.

[258] E.H. Kerner (Ed.), The Theory of Action-at-a-Distance in Relativistic Particle Dynamics. A Reprint Collection, in: International Science Review Series, vol. 11, Gordon and Breach, New York, 1972.

[259] P. Droz-Vincent, Relativistic systems of interacting particles, Phys. Scripta 2 (1970) 129-134.

[260] T. Gill, J. Lindesay, Canonical proper time formulation of relativistic particle dynamics, Internat. J. Theoret. Phys. 32 (11) (1993) $2087-2098$.

[261] P. Ramond, Action-at-a -distance theories and dual models, Phys. Rev. D 7 (2) (1973) 449-458.

[262] R. Marnelius, Lagrangian and Hamiltonian formulation of relativistic particle mechanics, Phys. Rev. D 10 (8) (1974) 2535-2553.

[263] R.P. Gaida, Yu.B. Klyuchkovskii, V.I. Tretyak, Lagrangian classical relativistic mechanics of a system of directly interacting particles. I, Theoret. Math. Phys. 44 (2) (1980) 687-697.

[264] R.P. Gaida, Yu.B. Klyuchkovskii, V.I. Tretyak, Lagrangian classical relativistic mechanics of a system of directly interacting particles. II, Theoret. Math. Phys. 45 (2) (1980) 963-975.

[265] R.P. Gaida, Yu.B. Klyuchkovskii, V.I. Tretyak, Forms of relativistic dynamics in a classical Lagrangian description of a system of particles, Theoret. Math. Phys. 55 (1) (1983) 372-384.

[266] S.N. Sokolov, V.I. Tretyak, Front form of relativistic Lagrangian dynamics in two-dimensional space time and its connection with the Hamiltonian description, Theoret. Math. Phys. 67 (1) (1986) 385-394.

[267] H. Sorge, H. Stöcker, W. Greiner, Poincare-invariant Hamiltonian dynamics: Modelling multi-hadronic interactions in a phase space approach, Ann. Phys. 192 (1989) 266-306.

[268] R.P. Gaida, V.I. Tretyak, Yu.G. Yaremko, Center-of-mass variables in the relativistic Lagrangian dynamics of a system of particles, Theoret. Math. Phys. $101(3)(1994) 1443-1453$

[269] P.A.M. Dirac, Forms of relativistic dynamics, Rev. Modern Phys. 21 (3) (1949) 392-399.

[270] J.L. Anderson, P.G. Bergmann, Constraints in covariant field theories, Phys. Rev. 83 (5) (1951) 1018-1025.

[271] I.T. Todorov, Dynamics of relativistic point particles as a problem with constraints, JINR-E2-10125, 1976, 26 pp.

[272] A. Komar, Constraint formalism of classical mechanics, Phys. Rev. D 18 (6) (1978) 1881-1886.

[273] A. Komar, Interacting relativistic particles, Phys. Rev. D 18 (6) (1978) 1887-1893.

[274] J.N. Goldberg, E.C.G. Sudarshan, N. Mukunda, Relativistically interacting particles and world lines, Phys. Rev. D 23 (10) (1981) $2231-2235$.

[275] A. Kihlberg, R. Marnelius, N. Mukunda, Relativistic potential models as systems with constraints and their interpretation, Phys. Rev. D 23 (10) (1981) 2201-2209.

[276] F. Rohrlich, Many-body forces and the cluster decomposition, Phys. Rev. D 23 (6) (1981) 1305-1315.

[277] F. Rohrlich, Evolution and covariance in constraint dynamics, Phys. Rev. D 25 (10) (1982) 2576-2583.

[278] J. Samuel, Constraints in relativistic Hamiltonian mechanics, Phys. Rev. D 26 (12)(1982) 3475-3481.

[279] L.P. Horwitz, F. Rohrlich, Constraint relativistic quantum dynamics, Phys. Rev. D 24 (6) (1981) 1528-1542.

[280] L.P. Horwitz, F. Rohrlich, Limitations of constraint dynamics, Phys. Rev. D 31 (4) (1985) 932-933.

[281] F. Marquès, V. Iranzo, A. Molina, A. Montoto, J. Llosa, World-line condition and the noninteraction theorem, Phys. Rev. D 31 (2) (1985) $314-318$.

[282] G. Longhi, L. Lusanna, Bound-state solutions, invariant scalar products, and conserved currents for a class of two-body relativistic systems, Phys. Rev. D 34 (12) (1986) 3707-3731.

[283] M. Montesinos, C. Rovelli, T. Thiemann, SL(2, R) model with two Hamiltonian constraints, Phys. Rev. D 60 (4) (1999) 044009

[284] Relativistic N-body problem in a separable two-body basis, Phys. Rev. C 63 (4) (2001) 044907.

[285] V. Gerdt, A. Khvedelidze, Yu. Palii, Towards an algorithmisation of the Dirac constraint formalism, in: J. Calmet, W.M. Seiler, R.W. Tucker (Eds.), Global Integrability of Field Theories. Proceedings of GIFT 2006, Cocroft Institute, Daresbury, UK, 2006, pp. 135-154.

[286] R. Hakim, H.D. Sivak, Relativistic statistical mechanics, a brief overview, in: AIP Conf. Proc., 841, 2006, pp. 63-99, A Century of Relativity Physics: ERE 2005; XXVIII Spanish Relativity Meeting.

[287] R. Becker, Theory of Heat, Springer, New York, 1967.

[288] K. Huang, Statistical Mechanics, Wiley, New York, 1963.

[289] M.H. Ernst, Nonlinear model-Boltzmann equations and exact solutions, Phys. Rep. 78 (1) (1981) 1-171

[290] C. Eckart, The thermodynamics of irreversible processes. III. Relativistic theory of the simple fluid, Phys. Rev. 58 (10) (1940) 919-924.

[291] A. Lichnerowicz, R. Marrot, Propriétés statistiques des ensembles de particules en relativité, C. R. Acad. Sci. Paris 210 (1940) 795-761.

[292] G.A. Kluitenberg, S.R. de Groot, P. Mazur, Relativistic thermodynamics of irreversible processes. I. Heat conduction, diffusion, viscous flow and chemical reactions; formal part, Physica 19 (1953) 689-704.

[293] S.T. Beliaev, G.I. Budker, Relativistic kinetic equation, Dokl. Akad. Nauk SSSR 6 (1956) 807-810. (English transl.: Soviet Phys. Dokl. 1 (1956), 218 ).

[294] W. Israel, Relativistic kinetic theory of a simple gas, J. Math. Phys. 4 (9) (1963) 1163-1181.

[295] Yu.L. Klimontovich, The Statistical Theory of Non-Equilibrium Processes in a Plasma, MIT Press, Cambridge, MA, 1967.

[296] A. Mangeney, Equations cinetiques pour un plasma homogene en interaction avec un champ de rayonnement, Physica 30 (3) (1964) $461-474$

[297] Ph. de Gottal, I. Prigogine, Relativistic effects in statistical hydrodynamics, Physica 31 (5) (1965) 677-687.

[298] R.W. Lindquist, Relativistic transport theory, Ann. Phys. 37 (1966) 487-518.

[299] H. Akama, Relativistic Boltzmann equation for plasmas, J. Phys. Soc. Japan 28 (2) (1970) 478-488. 
[300] S.R. de Groot, On relativistic kinetic theory of transport processes, in particular of neutrino systems, Ann. Inst. H. Poincaré (A) Phys. Théor. 31A (1979) 377-386.

[301] R.J. Gould, Kinetic theory of relativistic plasmas, Phys. Fluids 24 (1) (1981).

[302] D. Pavón, D. Jou, J. Casas-Vázquez, On a covariant formulation of dissipative phenomena, Ann. Inst. H. Poincaré (A) Phys. Théor. 36 (1) (1982) 79-88.

[303] C. Cercignani, G.M. Kremer, On relativistic collisional invariants, J. Statist. Phys. 96 (1-2) (1999) $439-445$.

[304] C. Cercignani, G.M. Kremer, Trend to equilibrium of a degenerate relativistic gas, J. Statist. Phys. 98 (1-2) (2000) 441-456

[305] M. Lemou, Linearized quantum and relativistic Fokker-Planck-Landau equations, Math. Methods Appl. Sci. 23 (12) (2000) 1093-1119.

306] M. Honda, Relativistic heat conduction and thermoelectric properties of nonuniform plasmas, Phys. Plasmas 10 (10)(2003) 4177-4180.

[307] R.M. Strain, Y. Guo, Stability of the relativistic Maxwellian in a collisional plasma, Comm. Math. Phys. 251 (2) (2004) $263-320$.

[308] L. Hsiao, H. Yu, Asymptotic stability of the relativistic Maxwellian, Math. Methods Appl. Sci. 29 (13)(2006) $1481-1499$.

[309] A. Bret, L. Gremillet, D. Benisti, E. Lefebvre, Exact relativistic kinetic theory of an electron-beam-plasma system: Hierarchy of the competing modes in the system-parameter space, Phys. Rev. Lett. 100 (2008) 205008.

[310] J.M. Stewart, Non-Equilibrium Relativistic Kinetic Theory, in: Lecture Notes in Physics, vol. 10, Springer, Berlin, 1971.

[311] S.R. de Groot, W.A. van Leeuwen, C.G. van Weert, Relativistic Kinetic Theory: Principles and Applications, North-Holland, Amsterdam, 1980.

[312] C. Cercignani, G.M. Kremer, The Relativistic Boltzmann Equation: Theory and Applications, in: Progress in Mathematical Physics, vol. 22, Birkhäuser Verlag, Basel, Boston, Berlin, 2002.

[313] J. Ehlers, in: R.K. Sachs (Ed.), General Relativity and Kinetic Theory, in: Proc. of the Int. School of Physics “Enrico Fermi”, Academic Press, New York, 1971, pp. 1-70. Course XLVII.

[314] H. Andréasson, The Einstein-Vlasov system/kinetic theory, Living Rev. Relativ. 8 (2) (2005).

[315] B. Svetitsky, Diffusion of charmed quarks in the quark-gluon plasma, Phys. Rev. D 37 (9) (1988) $2484-2491$.

[316] H. van Hees, R. Rapp, Thermalization of heavy quarks in the quark-gluon plasma, Phys. Rev. C 71 (3) (2005) 034907

[317] G. Wolschin, M. Biyajima, T. Mizoguchi, N. Suzuki, Time evolution of relativistic d + Au and Au + Au collisions, Ann. Phys. 15 (6) (2006) 369-378.

[318] H. van Hees, V. Greco, R. Rapp, Heavy-quark probes of the quark-gluon plasma and interpretation of recent data taken at the BNL relativistic heavy ion collider, Phys. Rev. C 73 (2006) 034913

[319] R. Rapp, V. Greco, H. van Hees, Heavy-quark spectra at RHIC and resonances in the QGP, Nuclear Phys. A 774 (2006) 685-688.

[320] M. Abdel-Aziz, S. Gavin, Causal diffusion and the survival of charge fluctuations in nuclear collisions, Phys. Rev. C 70 (3) (2004) 034905.

[321] M. Abdel-Aziz, S. Gavin, Causal diffusion at RHIC, J. Phys. G 31 (2005) S77-S84.

[322] G. Wolschin, Diffusion and local deconfinement in relativistic systems, Phys. Rev. C 69 (2) (2004) 024906

[323] C.D. Dermer, The production spectrum of a relativistic Maxwell-Boltzmann gas, Astrophys. J. 280 (1984) $328-333$.

[324] E. Bertschinger, Brownian motion of stars, dust, and invisible matter, AIP Conf. Proc. 861 (1) (2006) 97-105.

[325] B. Wolfe, F. Melia, Covariant kinetic theory with an application to the coma cluster, Astrophys. J. 638 (2006).

[326] M.E. Dieckmann, L. O'C. Drury, P.K. Shukla, On the ultrarelativistic two-stream instability, electrostatic turbulence and Brownian motion, New J. Phys. 8 (2006) 40.

[327] R. Aloisio, V. Berezinsky, A. Gazizov, Superluminal problem in diffusion of relativistic particles and its phenomenological solution. arXiv/08051867, 2008.

[328] P. Roy, J.E. Alam, S. Sarkar, B. Sinha, S. Raha, Quark-gluon plasma diagnostics in a successive equilibrium scenario, Nuclear Phys. A 624 (4) (1997) $687-705$.

[329] H. van Hees, V. Greco, R. Rapp, Thermalization and flow of heavy quarks in the quark-gluon plasma, AIP Conf. Proc. 842 (2006) 77-79.

[330] J. Łopuszaǹski, Relativisierung der theorie der stochastischen prozesse, Acta Phys. Polon. 12 (1953) 87-99.

[331] H. Rudberg, On the Theory of Relativistic Diffusion, Almquist and Wiksells, Uppsala, 1957.

[332] G. Schay, The equations of diffusion in the special theory of relativity, Ph.D. Thesis, Princeton University, 1961. Available through University Microfilms, Ann Arbor, MI, https://wwwlib.umi.com.

[333] R.M. Dudley, Lorentz-invariant Markov processes in relativistic phase space, Ark. Mat. Astron. Fys. 6 (1965) $241-268$

[334] R.M. Dudley, A note on Lorentz-invariant Markov processes, Ark. Mat. Astron. Fys. 6 (1967) 575-581.

[335] R.M. Dudley, Asymptotics of some relativistic Markov processes, Proc. Natl. Acad. Sci. USA 70 (12) (1973) $3551-3555$.

[336] R.M. Dudley, Recession of some relativistic Markov processes, Rocky Mountain J. Math. 4 (1967) 401-406.

[337] R. Hakim, A covariant theory of relativistic Brownian motion. I. Local equilibrium, J. Math. Phys. 6 (10) (1965) 1482-1495.

[338] R. Hakim, Relativistic stochastic processes, J. Math. Phys. 9 (1968) 1805-1818.

[339] Adolf Fick, Über diffusion, Poggendorff's Ann. Phys. 94 (1855) 59-86.

[340] K. Yasue, Derivation of relativistic wave equations in the theory of elementary domains, Progr. Theoret. Phys. 57 (1) (1977) $318-328$.

[341] J.-P. Caubet, Relativistic Brownian motion, in: Proceedings of the Conference on Probabilistic Methods in Differential Equations, University of Victoria, 1974, in: Lecture Notes in Mathematics, vol. 451, Springer, Berlin, 1975, pp. 131-142.

[342] J.P. Caubet, Mouvement Brownien spatiotemporal non isotrope, C. R. Acad. Sci. Paris 280 (1975) 303-306

[343] J.P. Caubet, Relativistic Brownian motion, Adv. Appl. Probab. 8 (2) (1976) 230-231.

[344] J.P. Caubet, Le Mouvement Brownian Relativiste, in: Lecture Notes in Mathematics, vol. 559, Springer, Berlin, Heidelberg, 1976.

[345] E. Nelson, Review of 'Le Mouvement Brownian Relativiste' by J. P. Caubet, Bull. Amer. Math. Soc. 84 (1978) 121-124.

[346] J.P. Caubet, Brownian motion, Minkowski space and principle of special relativity, C. R. Acad. Sci. Ser. A 284 (5) (1977) 339-341.

[347] P. Suppes, Probability in relativistic particle theory, Erkenntnis 16 (2)(1981) 299-305.

[348] F. Guerra, P. Ruggiero, A note on relativistic Markov processes, Lett. Nouvo Cimento 23 (1978) 529-534.

[349] F. Guerra, P. Ruggiero, New interpretation of the Euclidean-Markov field in the framework of physical Minkowski space-time, Phys. Rev. Lett. 31 (16) (1978) 1022-1025

[350] K. Namsrai, Relativistic dynamics of stochastic particles, Found. Phys. 10 (3-4) (1980) 353-361.

[351] S. Roy, Relativistic Brownian motion and the space-time approach to quantum mechanics, J. Math. Phys. 21 (1980) 71-76.

[352] S. Roy, Relativistic Brownian motion and the theory of measurement in quantum mechanics, Nuovo Cimento B 64 (1981) 81-93.

[353] J.P. Caubet, The Brownian theory of light, Stochastic Anal. Appl. 2 (1) (1984) 55-85.

[354] J.P. Caubet, The great fugue of the Brownian theory of light, Stochastic Anal. Appl. 3 (2) (1985) 119-151.

[355] E. Nelson, Probabilistic Methods in Mathematical Physics, World Scientific, Singapore, 1992, pp. 329-333.

[356] P. Garbaczewski, Relativistic problem of random flights and Nelson stochastic mechanics, Phys. Lett. A 164 (1) (1992) 6-16.

[357] L.M. Morato, L. Viola, Markov diffusions in comoving coordinates and stochastic quantization of the free relativistic particle, J. Math. Phys. 36 (1995) 4691-4710;

L.M. Morato, L. Viola, Markov diffusions in comoving coordinates and stochastic quantization of the free relativistic particle, J. Math. Phys. 37 (1996) 4769.

[358] P. Garbaczewski, J.R. Klauder, R. Olkiewicz, Schrödinger problem, Lévy processes, and noise in relativistic quantum mechanics, Phys. Rev. E 51 (5) (1995) 4114-4131.

[359] M. Nagasawa, Quantum theory theory of Brownian motions, and relativity theory, Chaos Solitons Fractals 7 (5) (1996) $631-643$.

[360] A. Posilicano, Poincareé invariant Markov processes and Gaussian random fields on relativistic phase-spaces, Lett. Math. Phys. 42 (1997) $85-93$.

[361] M. Pavon, On the stochastic mechanics of the free relativistic particle, J. Math. Phys. 42 (10) (2001) 4946-4856.

[362] J.R. Fanchi, Review of invariant time formulations of relativistic quantum theories, Found. Phys. 23 (3) (1993) 487-548

[363] E. Nelson, Derivation of the Schrödinger equation from Newtonian mechanics, Phys. Rev. 150 (4)(1966) 1079-1085.

[364] H. Grabert, P. Hänggi, P. Talkner, Is quantum mechanics equivalent to a classical stochastic process? Phys. Rev. A 19 (6) (1979) $2440-2445$.

[365] W. Thomson, On the theory of the electric telegraph, Proc. R. Soc. Lond. Ser. I 7 (1854-1855) 382-399. 
[366] S. Goldstein, On diffusion by discontinuous movements, and on the telegraph equation, Q. J. Mech. Appl. Math. 4 (2) (1951) $129-156$.

[367] M. Kac, A stochastic model related to the telegrapher's equation, Rocky Mountain J. Math. 4 (1974) 497-509.

[368] R. Fürth, Einige Untersuchungen über Brownsche Bewegungen an einem Einzelteilchen, Ann. Phys. (Berlin) 53 (1917) $177-213$.

[369] R. Fürth, Die Brownsche Bewegung bei Berücksichtigung einer Persistenz der Bewegungsrichtung. Mit Anwendungen auf die Bewegung lebender Infusorien, Z. Phys. 2 (1920) 244-256.

[370] G.I. Taylor, Diffusion by continuous movements, Proc. London Math. Soc. 20 (1922) 196-212.

[371] M. Boguñá, J.M. Porrà, J. Masoliver, Persistent random walk model for transport through thin slabs, Phys. Rev. E 59 (6) (1999) $6517-6526$.

[372] A. Ishimaru, Diffusion of light in turbid material, Appl. Optim. 28 (1989) 2210-2215.

[373] M. Miri, H. Stark, Persistent random walk in a honeycomb structure: Light transport in foams, Phys. Rev. E 68 (3) (2003) 031102.

[374] M. Miri, E. Madadi, H. Stark, Diffusive transport of light in a Kelvin foam, Phys. Rev. E 72 (3) (2005) 031111.

[375] M. Miri, Z. Sadjadi, M.E. Fouladvand, Persistent random walk on a site-disordered one-dimensional lattice: Photon subdiffusion, Phys. Rev. E 73 (3) (2006) 031115.

[376] H. Kim, C. Kim, E.K. Lee, P. Talkner, P. Hänggi, Wall-mediated self-diffusion in slit and cylindrical pores, Phys. Rev. E 77 (2008) 031202.

[377] D.D. Joseph, L. Preziosi, Heat waves, Rev. Modern Phys. 61 (1) (1989) 41-73.

[378] D.D. Joseph, L. Preziosi, Addendum to the paper "Heat waves" [Rev. Mod. Phys. 61, 41 (1989)], Rev. Modern Phys. 62 (2) (1990) 375-391.

[379] P. Kostädt, M. Liu, Causality and stability of the relativistic diffusion equation, Phys. Rev. D 62 (2000) 023003.

[380] P. Kostädt, M. Liu, Alleged acausality of the diffusion equations: A reply, Phys. Rev. D 64 (2001) 088504.

[381] L. Herrera, D. Pavón, Why hyperbolic theories of dissipation cannot be ignored: Comment on a paper by Kostädt and Liu, Phys. Rev. D 64 (2001) 088503.

[382] M.E. Peskin, D.V. Schroeder, An Introduction to Quantum Field Theory, Perseus Books, Reading, MA, 1995.

[383] B. Gaveau, T. Jacobson, M. Kac, L.S. Schulman, Relativistic extension of the analogy between quantum mechanics and Brownian motion, Phys. Rev. Lett. 53 (5) (1984) 419-422.

[384] T. Ichinose, Path integral for the Dirac equation in two space-time dimensions, Proc. Japan Acad. 58 (1982) $290-293$.

[385] T. Ichinose, Path integral formulation of the propagator for a two-dimensional Dirac particle, Physica A 124 (1-3) (1984) $419-425$.

[386] T. Jacobson, L.S. Schulman, Quantum stochastics: The passage from a relativistic to a non-relativistic path integral, J. Phys. A 17 (1984) $375-383$.

[387] A.O. Barut, I.H. Duru, Path-integral derivation of the Dirac propagator, Phys. Rev. Lett. 53 (25) (1984) 2355-2358.

[388] B. Gaveau, L.S. Schulman, Grassmann-valued processes for the Weyl and the Dirac equations, Phys. Rev. D 36 (4) (1987) 1135-1140.

[389] R.P. Feynman, A.R. Hibbs, Quantum Mechanics and Path Integrals, McGraw-Hill, New York, 1965, problem 2-6, pp. 34-36.

[390] W.L. Kath, Waiting and propagating fronts in nonlinear diffusion, Physica D 12 (1984) 375-381.

[391] O. Oron, L.P. Horwitz, Relativistic Brownian motion and gravity as an Eikonal approximation to a quantum evolution equation, Found. Phys. 35 (7) (2005) 1181-1203.

[392] F. Castejón, S. Eguilior, Langevin equations for quasi-linear wave-particle interaction, Plasma Phys. Control. Fusion 45 (2) (2003) 159-167.

[393] A. Cabo-Bizet, A. Cabo Montes de Oca, Particle dynamics in a relativistic invariant stochastic medium, Phys. Lett. A 359 (2006) 265-271.

[394] R. Zygadło, Two Markovian models leading to the Pascalian (1D ultrarelativistic) stationary distribution, Phys. Lett. A 329 (2004) 459-463.

[395] A. Fingerle, Relativistic fluctuation theorems: Theory and explicit examples, C. R. Acad. Sci. Physique 8 (5-6) (2007) 696-713. arXiv:0705.3115v1.

[396] P. Danielewicz, Nonrelativistic and relativistic Landau/Fokker-Planck equations for arbitrary statistics, Physica 100A (1980) $167-182$.

[397] H. van Erkelens, Relativistic Boltzmann theory for a plasma. VI. The relativistic Landau equation, Physica 107A (1981) 48-70.

[398] N.J. Fisch, Current generation in a relativistic plasma, Phys. Rev. A 24 (6) (1981) 3245-3248.

[399] C.F.F. Karney, N.J. Fisch, Efficiency of current drive by fast waves, Phys. Fluids 28 (1) (1985) 116-126.

[400] P. de Gottal, J. Gariel, Test-particle motion in a relativistic plasma. I. Weakly coupled medium, Physica A 157 (1989) $1059-1073$.

[401] Ph. de Gottal, J. Gariel, Test-particle motion in a relativistic plasma. II. The Lorentz gas, Physica A 163 (1990) $615-624$.

[402] Ph. de Gottal, J. Gariel, Test-particle motion in a relativistic plasma. III. Collective effects, Physica A 186 (1992) 534-548.

[403] O.V. Klimov, A.A. Tel'nikhin, Stochastic dynamics of a relativistic particle in a wave-packet field, Plasma Phys. Rep. 21 (1995) 335-338.

[404] J.R. Davies, A.R. Bell, M.G. Haines, S.M. Guérin, Short-pulse high-intensity laser-generated fast electron transport into thick solid targets, Phys. Rev. E 56 (6) (1997) 7193-7203.

[405] K.C. Baral, J.N. Mohanty, Covariant formulation of the Fokker-Planck equation for moderately coupled relativistic magnetoplasma, Phys. Plasmas 7 (2000) 1103-1112.

[406] K.C. Baral, J.N. Mohanty, Relativistic theory of moderately coupled plasmas: Formulation of appropriate Fokker-Planck equation, Phys. Plasmas 9 (11) (2002) 4507-4511.

[407] T. Yokota, Y. Nakao, T. Johzaki, K. Mima, Two-dimensional relativistic Fokker-Planck model for core plasma heating in fast ignition targets, Phys. Plasmas 13 (1) (2006) 022702

[408] G.V. Khazanov, A.A. Tel'nikhina, T.K. Kronberg, Chaotic motion of relativistic electrons driven by whistler waves, Plasma Phys. Control. Fusion 49 (4) (2007) 447-466.

[409] P.J.D. Mauas, D.O. Gomez, Kinetic description of electron beams in the solar chromosphere, Astophys. J. 398 (1997) 682-691.

[410] P.J.D. Mauas, D.O. Gomez, Fokker-Planck Description of Electron Beams in the Solar Chromosphere, Astophys. J. 483 (1997) $496-506$.

[411] N. Itoh, Y. Kohyama, S. Nozawa, Relativistic corrections to the Sunyaev-Zel'dovich effect for clusters of galaxies, Astrophys. J. 502 (1998) 7-15.

[412] P.A. Becker, T. Le, C.D. Dermer, Time-dependent stochastic particle acceleration in astrophysical plasmas: Exact solutions including momentumdependent escape, Astrophys. J. 647 (1) (2006) 539-551.

[413] T.H. Boyer, Inconsistency between the Boltzmann distribution for relativistic free particles and the Rayleigh-Jeans law for thermal radiation within classical electrodynamics, Phys. Rev. D 19 (1979) 1112-1115.

[414] T.H. Boyer, Inconsistency between the Boltzmann distribution for relativistic free particles and the Planck spectrum for thermal radiation in quantum theory, Phys. Rev. D 19 (1979) 3635-3640.

[415] U. Ben-Ya'acov, Relativistic Brownian motion and the spectrum of thermal radiation, Phys. Rev. D 23 (6) (1981) 1441-1450.

[416] R. Blanco, L. Pesquera, E. Santos, Equilibrium between radiation and matter for classical relativistic multiperiodic systems. Derivation of Maxwell-Boltzmann distribution from Rayleigh-Jeans spectrum, Phys. Rev. D 27 (6) (1983) 1254-1287.

[417] C.F.F. Karney, Fokker-Planck and quasilinear codes, Comput. Phys. Rep. 4 (3-4) (1986) 183-244.

[418] M. Shoucri, V. Fuchs, A. Bers, The application of the finite-element library TWODEPEP for the numerical solution of the relativistic Fokker-Planck equation, Comput. Phys. Comm. 46 (3) (1987) 337-344.

[419] Y. Peysson, M. Shoucri, An approximate factorization procedure for solving nine-point elliptic difference equations - Application for a fast 2-D relativistic Fokker-Planck solver, Comput. Phys. Comm. 109 (1) (1998) 55-80.

[420] P.E. Kloeden, E. Platen, Numerical Solution of Stochastic Differential Equations, in: Stochastic Modelling and Applied Probability, vol. 23, Springer, 2006.

[421] R. Rapp, H. van Hees, Heavy quark diffusion as a probe of the quark-gluon plasma, 2008, arXiv:0803.0901v2.

[422] C.W. Gardiner, Handbook of Stochastic Methods, 2nd ed., in: Springer Series in Synergetics, Springer, Berlin, 2002.

[423] W. Ebeling, Nonlinear Brownian motions - mean square displacement, Condens Matter Phys. 7 (3(39)) (2004) 539-550.

[424] P. Hänggi, Stochastic Processes I: Asymptotic behaviour and symmetries, Helv. Phys. Acta 51 (1978) $183-201$.

[425] W. Ebeling, Canonical non-equilibrium statistics and applications to Fermi-Bose systems, Condens Matter Phys. 3 (2) (2000) $285-293$.

[426] P.S. Epstein, On the resistance experienced by spheres in their motion through gases, Phys. Rev. 23 (1924) 710.

[427] M.R. Hoare, The linear gas, Adv. Chem. Phys. 20 (1971) 135-214.

[428] M.R. Hoare, S. Raval, M. Rahman, The Rayleigh model: Singular transport theory in one dimension, Philos. Trans. R. Soc. Lond. A 305 (1982) $383-440$.

[429] S.A. Trigger, Fokker-Planck equation for Boltzmann-type and active particles: Transfer probability approach, Phys. Rev. E 67 (2003) 046403. 
[430] R. Zwanzig, Nonlinear generalized Langevin equations, J. Statist. Phys. 9 (5) (1973) 215-220.

[431] R. Benzi, A. Sutera, A. Vulpiani, The mechanism of stochastic resonance, J. Phys. A 14 (1981) L453-L457.

[432] R. Benzi, G. Parisi, A. Sutera, A. Vulpiani, A theory of stochastic resonance in climatic change, SIAM J. Appl. Math. 43 (1983) $565-578$.

[433] C. Nicolis, G. Nicolis, Stochastic aspects of climatic transitions - Additive fluctuations, Tellus 33 (1981) 225-234.

[434] C. Nicolis, Long-term climatic transitions and stochastic resonance, J. Statist. Phys. 70 (1/2)(1993) 3-13.

[435] L. Gammaitoni, P. Hänggi, P. Jung, F. Marchesoni, Stochastic resonance, Rev. Modern Phys. 70 (1) (1998) $223-287$

[436] A. Neiman, L. Schimansky-Geier, Stochastic resonance in bistable systems driven by harmonic noise, Phys. Rev. Lett. 72 (19) (1994) $2988-2991$.

[437] V.S. Anishchenko, A.B. Neiman, F. Moss, L. Schimansky-Geier, Stochastic resonance: Noise-enhanced order, Sov. Phys. Usp. 42 (January) (1999) 7-36.

[438] B. Lindner, L. Schimansky-Geier, Coherence and stochastic resonance in a two-state system, Phys. Rev. E 61 (6) (2000) $6103-6110$.

[439] B. Lindner, J. Garca-Ojalvo, A. Neiman, L. Schimansky-Geier, Effects of noise in excitable systems, Phys. Rep. 392 (6) (2004) $321-424$.

[440] P. Jung, P. Hänggi, F. Marchesoni, Colored-noise-driven bistable systems, Phys. Rev. A 40 (9) (1989) 5447-5450.

[441] P. Jung, P. Hänggi, Amplification of small signals via stochastic resonance, Phys. Rev. A 44 (12) (1991) 8032-8042.

[442] J. Blum, S. Bruns, D. Rademacher, A. Voss, B. Willenberg, M. Krause, Measurement of the translational and rotational Brownian motion of individual particles in a rarefied gas, Phys. Rev. Lett. 97 (2006) 230601.

[443] A.S. Kompaneets, The establishment of thermal equilibrium between quanta and electrons, Sov. Phys. JETP 4 (5) (1957) $730-737$.

[444] R.L. Liboff, Kinetic Theory, Prentice Hall Inc., Englewood Cliffs, New Jersey, 1990, p. 07632.

[445] P. Hänggi, Langevin description of Markovian integro-differential master equations, Z. Phys. 36 (1980) $271-282$.

[446] P. Hänggi, Langevin description of Markov master equations II: Noise correlations, Z. Phys. B 43 (1981) 269-273.

[447] P. Hänggi, H. Grabert, P. Talkner, H. Thomas, Bistable systems: Master equation versus Fokker-Planck modeling, Phys. Rev. A 29 (1984) $371-378$.

[448] J. Dunkel, Relativistic Brownian motion and diffusion processes, Ph.D. Thesis, Universität Augsburg, July 2008.

[449] Yu.L. Klimontovich, A.S. Kovalev, P.S. Landa, Natural fluctuations in lasers, Phys.-Uspekhi 15 (1) (1972) 95-113

[450] S. Marksteiner, K. Ellinger, P. Zoller, Anomalous diffusion and Lévy walks in optical lattices, Phys. Rev. A 53 (1996) $3409-3430$.

[451] E. Lutz, Anomalous diffusion and Tsallis statistics in an optical lattice, Phys. Rev. A 67 (2003) 051402.

[452] S.A. Khrapak, A.V. Ivlev, G.E. Morfill, S.K. Zhdanov, Scattering in the attractive Yukawa potential in the limit of strong interaction, Phys. Rev. Lett. 90 (22) (2003) 225002

[453] S.A. Khrapak, A.V. Ivlev, G.E. Morfill, Momentum transfer in complex plasmas, Phys. Rev. E 70 (2004) 056405

[454] S.A. Trigger, W. Ebeling, A.M. Ignatov, I.M. Tkachenko, Fokker-Planck equation with velocity-dependent coefficients: Application to dusty plasmas and active particles, Contrib. Plasma Phys. 43 (2003) 377.

[455] A.G. Zagorodny, P.P.J.M. Schram, S.A. Trigger, Stationary velocity and charge distributions of grains in dusty plasmas, Phys. Rev. Lett. 84 (2000) 3594-3597.

[456] A.S. Mikhailov, Selected topics in fluctuational kinetics of reactions, Phys. Rep. 184 (5-6) (1989) 307-374

[457] M. Schienbein, H. Gruler, Langevin equation, Fokker-Planck equation and cell migration, Bull. Math. Biol. 55 (1993) 585-608.

[458] W. Ebeling, F. Schweitzer, B. Tilch, Active Brownian particles with energy depots modelling animal mobility, Biosystems 49 (1999) 17-29.

[459] U. Erdmann, W. Ebeling, L. Schimansky-Geier, F. Schweitzer, Brownian particles far from equilibrium, Eur. Phys. J. B 15 (2000) $105-113$.

[460] F. Schweitzer, W. Ebeling, B. Tilch, Complex motion of Brownian particles with energy depots, Phys. Rev. Lett. 80 (23) (1998) 5044-5047.

[461] F. Schweitzer, W. Ebeling, B. Tilch, Statistical mechanics of canonical-dissipative systems and applications to swarm dynamics, Phys. Rev. E 64 (2001) 021110.

[462] W. Ebeling, J. Dunkel, U. Erdmann, S.A. Trigger, Klimontovich's contributions to the kinetic theory of nonlinear Brownian motion and new developments, J. Phys.: Conf. Ser. 11 (2005) 89-98.

[463] W. Ebeling, U. Erdmann, J. Dunkel, M. Jenssen, Nonlinear dynamics and fluctuations of dissipative Toda chains, J. Statist. Phys. 101 (1/2) (2000) $443-457$.

[464] W. Ebeling, P.S. Landa, V. Ushakov, Self-oscillations in Toda ring chains with negative friction, Phys. Rev. E 63 (2001) 046601.

[465] V.A. Makarov, E. del Rio, W. Ebeling, M.G. Velarde, Dissipative Toda-Rayleigh lattice and its oscillatory modes, Phys. Rev. E 64 (2001) 036601

[466] J. Dunkel, W. Ebeling, U. Erdmann, V.A. Makarov, Coherent motions and clusters in a dissipative Morse ring chain, Internat. J. Bifur. Chaos 12 (11) (2002) 2359-2377.

[467] S. Jespersen, R. Metzler, H.C. Fogedby, Lévy flights in external force fields: Langevin and fractional Fokker-Planck equations and their solutions, Phys. Rev. E 59 (3) (1999) 2736-2745.

[468] I.M. Sokolov, Lévy flights from a continuous-time process, Phys. Rev. E 63 (1) (2000) 011104

[469] A. Weron, K. Burnecki, S. Mercik, K. Weron, Complete description of all self-similar models driven by Lévy stable noise, Phys. Rev. E 71 (1) (2005) 016113.

[470] S.I. Denisov, W. Horsthemke, P. Hänggi, Steady-state Levy flights in a confined domain, Phys. Rev. E 77 (2008) 061112.

[471] B. Dybiec, E. Gudowska-Nowak, P. Hänggi, Lévy-Brownian motion on finite intervals: Mean first passage time, Phys. Rev. E 73 (2006) 046104.

[472] J.M. Sancho, A.M. Lacasta, K. Lindenberg, I.M. Sokolov, A.H. Romero, Diffusion on a solid surface: Anomalous is normal, Phys. Rev. Lett. 92 (25) (2004) 250601.

[473] E. Heinsalu, M. Patriarca, I. Goychuk, G. Schmid, P. Hänggi, Fractional Fokker-Planck dynamics: Numerical algorithm and simulations, Phys. Rev. E 73 (4) (2006) 046133.

[474] I. Goychuk, E. Heinsalu, M. Patriarca, G. Schmid, P. Hänggi, Current and universal scaling in anomalous transport, Phys. Rev. E 73 (2) (2006) 020101.

[475] J. Dunkel, P. Talkner, P. Hänggi, Relative entropy, Haar measures and relativistic canonical velocity distributions, New J. Phys. 9 (2007) 144.

[476] A. Gamba, Physical quantities in different reference systems according to relativity, Amer. J. Phys. 35 (2) (1967) 83-89.

[477] W. Israel, Thermodynamics of relativistic systems, Physica 106A (1981) 204-214.

[478] P.R. Halmos, Measure Theory, in: The University Series in Higher Mathematics, D. Van Nostrand Comp Inc., New York, 1951.

[479] I. Campos, J.L. Jiménez, Comment on the 4/3 problem in the electromagnetic mass and the Boyer-Rohrlich controversy, Phys. Rev. D 33 (2) (1986) 607-610.

[480] P.A.M. Dirac, The conditions for statistical equilibrium between atoms, electrons and radiation, Proc. R. Soc. Lond. A 106 (1924) 581-596.

[481] I. Müller, Max Planck - A life for thermodynamics, Ann. Phys. 17 (2-3) (2008) 73-87.

[482] H. Callen, Thermodynamics as a science of symmetry, Found. Phys. 4 (4) (1974) 423-442.

[483] R.A. Sunyaev, Y.B. Zeldovich, The observations of relic radiation as a test of the nature of x-ray radiation from the clusters of galaxies, Comm. Astrophys. Space Phys. 4 (1972) 173.

[484] M. Birkinshaw, S.F. Gull, H. Hardebeck, The Sunyaev-Zel'dovich effect towards three clusters of galaxies, Nature 309 (1984) 34-35.

[485] M. Jones, et al., An image of the Sunyaev-Zel'dovich effect, Nature 365 (1993) 320-323.

[486] M. Abramowitz, I.A. Stegun (Eds.), Handbook of Mathematical Functions, Dover Publications Inc., New York, 1972.

[487] W. Ochs, Basic properties of the generalized Boltzmann-Gibbs-Shannon entropy, Rep. Math. Phys. 9 (2) (1976) $135-155$.

[488] W. Ochs, A unique characterization of the generalized Boltzmann-Gibbs-Shannon entropy, Rep. Math. Phys. 9 (3) (1976) 331-354

[489] S. Kullback, R.A. Leibler, On information and sufficiency, Ann. Math. Statist. 22 (1951) 79-86.

[490] A. Wehrl, General properties of entropy, Rev. Modern Phys. 50 (2) (1978) 221-260.

[491] A. Wehrl, The many facets of entropy, Rep. Math. Phys. 30 (1) (1991) 119-129.

[492] M. Campisi, Thermodynamics with generalized ensembles: The class of dual orthodes, Physica A 385 (2) (2007) $501-517$.

[493] A. Montakhab, M. Ghodrat, M. Barati, Statistical thermodynamics of a two dimensional relativistic gas. arXiv:0809.1517v1, 2008.

[494] Yu.M. Sinyukov, On the relativistic partition function of ideal gases, Phys. Lett. B 127 (6) (1983) $443-447$.

[495] J. Franchi, Y. Le Jan, Relativistic diffusions and Schwarzschild geometry, Comm. Pure Appl. Math. 60 (2006) $187-251$.

[496] L. Zaninetti, X-ray profiles in symmetric and asymmetric supernova remnants, Baltic Astron. 16 (2007) $251-285$. 
[497] F. Debbasch, Relativistic stochastic processes: A review, AIP Conf. Proc. 861 (2006) 488-493.

[498] M. Rigotti, A relativistic stochastic process, Master's Thesis, ETH Zürich, Institute for Theoretical Physics, 2005.

[499] P. Hänggi, Connection between deterministic and stochastic descriptions of nonlinear systems, Helv. Phys. Acta 53 (1980) $491-496$.

[500] P.E. Protter, Stochastic Integration and Differential Equations, Springer-Verlag, New York, 2004.

[501] L.H. Ryder, Quantum Field Theory, 2nd edition, Cambridge University Press, Cambridge, 1996.

[502] G. Roepstorff, Path Integral Approach to Quantum Mechanics, Springer, Berlin, 1996.

[503] D. Forster, Hydrodynamics, Fluctuations, Broken Symmetry, and Correlation Functions, in: Frontiers in Physics, The Benjamin/Cummings Publishing Company, Inc., Reading, MA, 1975.

[504] R. Graaff, K. Rinzema, Practical improvements on photon diffusion theory: Application to isotropic scattering, Phys. Med. Biol. 46 (2001) $3043-3050$.

[505] D.C. Sahni, E.B. Dahl, N.G. Sjöstrand, Diffusion coefficient for photon transport in turbid media, Phys. Med. Biol. 48 (2003) $3969-3976$.

[506] J. Masoliver, J.M. Porrà, G.H. Weiss, Solutions of the telegrapher's equation in the presence of traps, Phys. Rev. A 45 (4) (1992) $2222-2227$.

[507] J. Masoliver, J.M. Porrà, G.H. Weiss, Erratum: Solutions of the telegrapher's equation in the presence of traps, Phys. Rev. A 46 (1992) 3574.

[508] S.K. Foong, Path integral solutions for telegrapher equation, in: Lectures on Path Integration, World Scientific, Singapore, 1993, pp. 427-449.

[509] S.K. Foong, S. Kanno, Comment on "Solutions of the telegrapher's equation in the presence of traps", Phys. Rev. A 46 (8) (1992) $5296-5297$.

[510] M. Renardy, Asymptotic solution of the telegraph equation (Mark A. Pinsky), SIAM Rev. 35 (2) (1993) 306-307.

[511] A.Y. Dorogovtsev, Periodic in distribution solution for a telegraph equation, J. Appl. Math. Stoch. Anal. 12 (2)(1999) $121-131$.

[512] M.O. Caceres, Non-Markovian processes with long-range correlations: Fractal dimension analysis, Braz. J. Phys. 29 (1) (1999) 125-135.

[513] P.A.M. Dirac, Classical theory of radiating electrons, Proc. R. Soc. Lond. Ser. A 167 (929) (1938) 148-169.

[514] C.J. Eliezer, On the classical theory of particles, Proc. R. Soc. Lond. 194 (1948) 543-555.

[515] F. Rohrlich, Classical self-force, Phys. Rev. D 60 (8) (1999) 084017.

[516] F. Rohrlich, The self-force and radiation reaction, Amer. J. Phys. 68 (12) (2000) 1109-1112.

[517] H. Spohn, The critical manifold of the Lorentz-Dirac equation, Europhys. Lett. 50 (2000) 287-292.

[518] G. Ares de Parga, R. Mares, S. Dominguez, An unphysical result for the Landau-Lifshitz equation of motion for a charged particle, Rev. Mexicana Fis. 52 (2) (2006) 139-142.

[519] P.R. Johnson, B.L. Hu, Stochastic theory of relativistic particles moving in a quantum field: I, Influence Functional and Langevin Equation. arXiv:quant$\mathrm{ph} / 0012137 \mathrm{v} 4,2001$.

[520] P.R. Johnson, B.L. Hu, Worldline influence functional: Abraham-Lorentz-Dirac-Langevin equation from QED, in: P. Chen (Ed.), Quantum Aspects of Beam Physics, in: 18th Advanced IFCA Beam Dynamics Workshop, Capri 2000, World Scientific, Singapore, 2002, pp. 95-104.

[521] P.R. Johnson, B.L. Hu, Stochastic theory of relativistic particles moving in a quantum field: Radiation reaction, vacuum fluctuations, and the Abraham-Lorentz-Dirac-Langevin equation, Phys. Rev. D 65 (2002) 065015.

[522] C.R. Galley, B.L. Hu, S. Lin, Electromagnetic and gravitational self-force on a relativistic particle from quantum fields in curved space, Phys. Rev. D 74 (2) (2006) 024017.

[523] E. Fermi, On the origin of the cosmic radiation, Phys. Rev. 75 (8) (1949) 1169-1174.

[524] C. Jarzynski, Nonequilibrium equality for free energy differences, Phys. Rev. Lett. 78 (14) (1997) $2690-2693$.

[525] U. Seifert, Entropy production along a stochastic trajectory and an integral fluctuation theorem, Phys. Rev. Lett. 95 (2005) 040602.

[526] F. Douarche, S. Joubaud, N.B. Garnier, A. Petrosyan, S. Ciliberto, Work fluctuation theorems for harmonic oscillators, Phys. Rev. Lett. 97 (14) (2006) 140603.

[527] C. Jarzynski, Nonequilibrium fluctuations of a single biomolecule, in: Controlled Nanoscale Motion, in: Lecture Notes in Physics, vol. 711, Springer, Berlin, Heidelberg, 2007, pp. 201-216.

[528] P. Talkner, E. Lutz, P. Hänggi, Fluctuation theorems: Work is not an observable, Phys. Rev. E 75 (5) (2007) 050102.

[529] P. Talkner, P. Hänggi, M. Morillo, Microcanonical quantum fluctuation theorems, Phys. Rev. E 77 (2008) 051131.

[530] B.L. Hu, E. Verdaguer, Stochastic gravity: Theory and applications, Living Rev. Relativ. 7 (3) (2004).

[531] H.-P. Breuer, F. Petruccione, A Lorentz covariant stochastic wave function dynamics for open systems, Phys. Lett. A 242 (4-5) (1998) $205-210$.

[532] H.-P. Breuer, F. Petruccione, Relativistic formulation of quantum state diffusion, J. Phys. A 31 (1) (1998) 33-52.

[533] H.-P. Breuer, F. Petruccione, Reply to 'Relativistic formulation of quantum state diffusion?', J. Phys. A 31 (47) (1998) 9605-9612.

[534] L. Diosi, Relativistic formulation of quantum state diffusion? J. Phys. A 31 (47) (1998) 9601-9603.

[535] H. Kleinert, S.V. Shabanov, Theory of Brownian motion of a massive particle in spaces with curvature and torsion, J. Phys. A 31 (1998) $7005-7009$.

[536] D.L. Rapoport, On the unification of geometric and random structures through torsion fields: Brownian motion, viscous and magneto-fluid-dynamics, Found. Phys. 35 (7) (2005) 1205-1243.

[537] D.L. Rapoport, Cartan-Weyl Dirac and Laplacian operators, Brownian motions: The quantum potential and scalar curvature, Maxwell's and Dirac-Hestenes equations, and supersymmetric systems, Found. Phys. 35 (8) (2005) 1385-1431.

[538] C. Chevalier, F. Debbasch, Fluctuation-dissipation theorems in an expanding universe, J. Math. Phys. 48 (2) (2007) 023304.

[539] F. Debbasch, A diffusion process in curved space-time, J. Math. Phys. 45 (7) (2004) 2744-2760.

[540] M. Rigotti, F. Debbasch, An H-theorem for the general relativistic Ornstein-Uhlenbeck process, J. Math. Phys. 46 (2005) 103303.

[541] H.B. Callen, Thermodynamics and an Introduction to Thermostatistics, 2nd edition, John Wiley \& Sons, New York, 1985.

[542] R.J.J. Jongschaap, H.C. Öttinger, Equilibrium thermodynamics, J. Non-Newtonian Fluid. Mech. 96 (2001) 5-17.

[543] A. Sommerfeld, Thermodynamik und Statistik, in: Vorlesungen über Theoretische Physik, vol. V, Verlag Harri Deutsch, Frankfurt/M., 1977.

[544] R.E. Gamboa-Saravi, The electromagnetic energy-momentum tensor, J. Phys. A 35 (2002) 9199-9203. 